\title{
ARL STATISTICS 2008-2009
}

Compiled and Edited by

MARTHA KYRILLIDOU

SHANEKA MORRIS

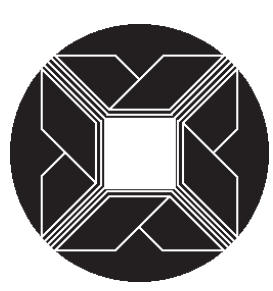

Association of ReseARCH LibRARIES WASHINGTON, DC 2011 
ARL Statistics 2008-2009

The tables presented in this publication are not indicative of performance and outcomes and should not be used as measures of library quality. In comparing any individual library to ARL medians or to other ARL members, one must be careful to make such comparisons within the context of differing institutional and local goals and characteristics.

The ARL Statistics datafiles and accompanying documentation are available at www.arl.org/stats/annualsurveys/arlstats/.

Published by the

Association of Research Libraries

Washington, DC 20036

www.arl.org

ISSN 0147-2135 print

ISSN 1943-5983 online

ISBN 1-59407-859-9

EAN 978-159407-859-0

\section{(C) 2011}

The compilation is copyrighted by the Association of Research Libraries. Blanket permission is granted to reproduce and distribute copies of this work for nonprofit, educational, or library purposes, provided that the author, source, and copyright notice are included on each copy. This permission is in addition to rights of reproduction granted under Sections 107, 108, and other provisions of the US Copyright Act. 


\section{CONTENTS}

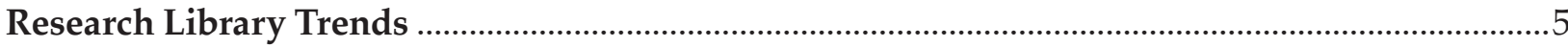

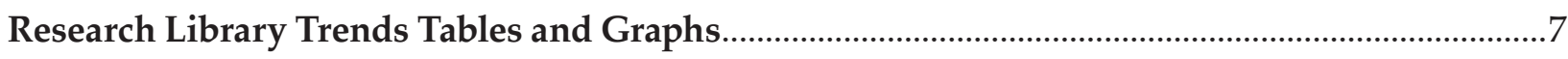

Table 1 Service Trends in ARL Libraries, 1991-2009 .......................................................................

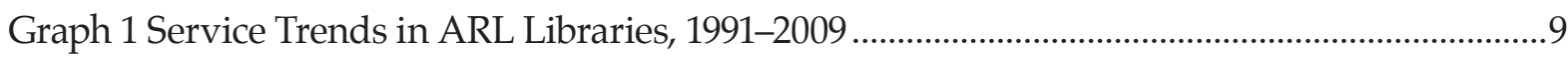

Table 2 Monograph and Serial Expenditures in ARL Libraries, 1986-2009 ..................................10

Graph 2 Monograph and Serial Expenditures in ARL Libraries, 1986-2009*................................11

Table 3 Supply and Demand in ARL Libraries, 1986-2009................................................................12

Graph 3 Supply and Demand in ARL Libraries, 1986-2009* ........................................................13

Table 4 Expenditure Trends in ARL Libraries, 1986-2009 ............................................................... 14

Graph 4 Expenditure Trends in ARL Libraries, 1986-2009 .............................................................15

Table 5 Resources per Student in ARL University Libraries, 1986-2009 .......................................... 16

Graph 5 Resources per Student in ARL University Libraries, 1986-2009 ......................................17

Table 6 Electronic Resources Expenditures in ARL University Libraries, 2008-2009 ...................18

Graph 6 Electronic Resources vs. Total Materials Expenditures, 1993-2009..................................19

Table 7a Electronic Resources and Materials Expenditures in

ARL University Libraries, 1992-2002 ….............................................................................................20

Table 7b Electronic Resources and Materials Expenditures in

ARL University Libraries, 2002-2009 ...............................................................................................21

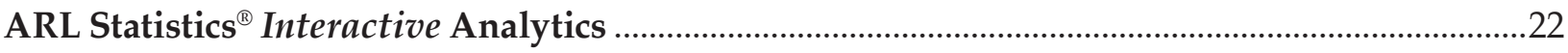

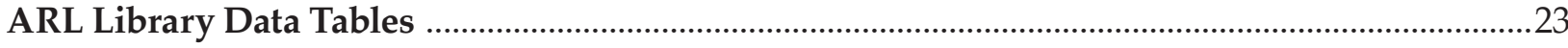

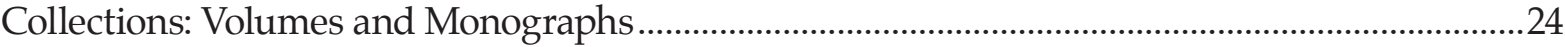

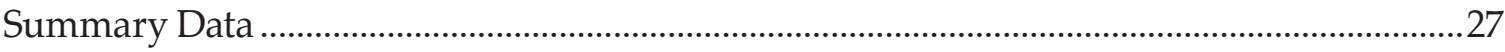

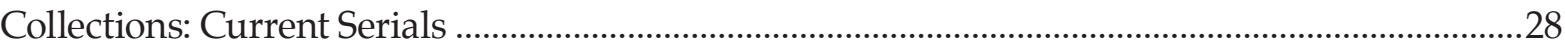

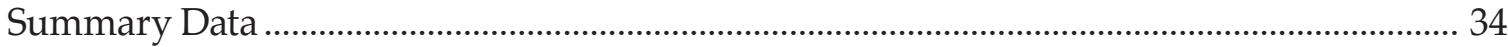

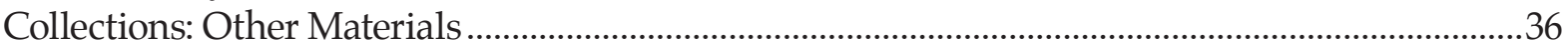

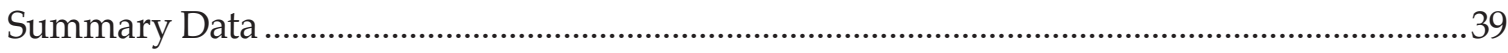

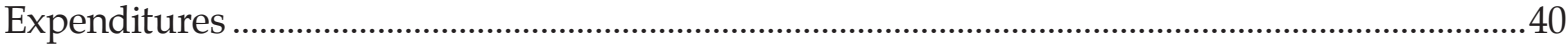

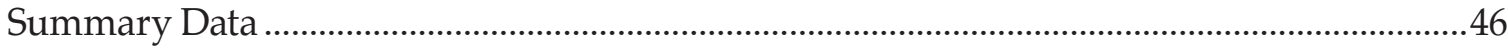

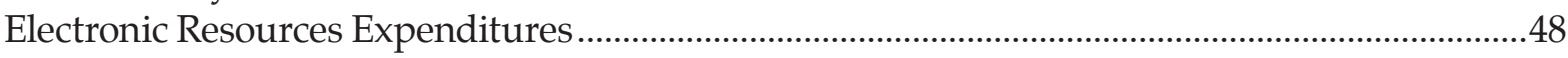

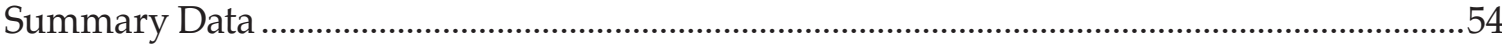

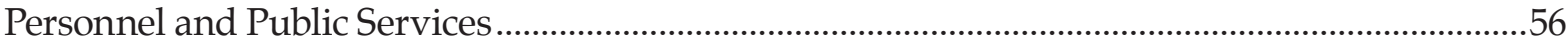

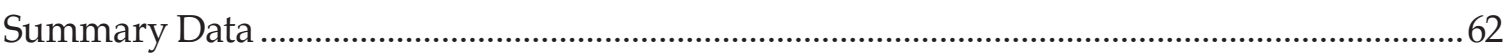


PhD, Faculty, and Enrollment Statistics. 65

Summary Data 68

Rank Order Tables of University Libraries 2008-2009 69

Summary of Rank Order Tables for University Libraries, 2008-2009 ...........................................70

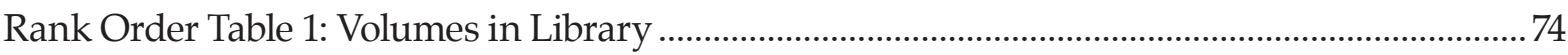

Rank Order Table 2: Volumes Added (Gross) …...................................................................................75

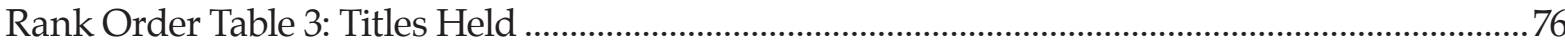

Rank Order Table 4: Current Serials (Total) ....................................................................................77

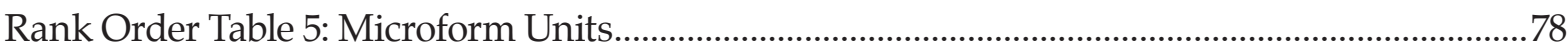

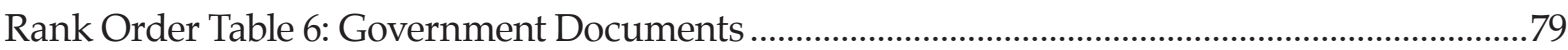

Rank Order Table 7: Total Library Materials Expenditures ….........................................................80

Rank Order Table 8: Total Salaries \& Wages Expenditures................................................................81

Rank Order Table 9: Other Operating Expenditures .........................................................................82

Rank Order Table 10: Total Library Expenditures .........................................................................83

Rank Order Table 11: Monographs Purchased (Volumes)............................................................. 84

Rank Order Table 12: Expenditures for Monographs ........................................................................85

Rank Order Table 13: Current Serials Purchased (Titles).................................................................86

Rank Order Table 14: Expenditures for Current Serials .................................................................8

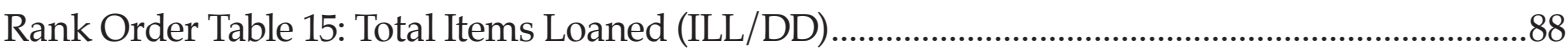

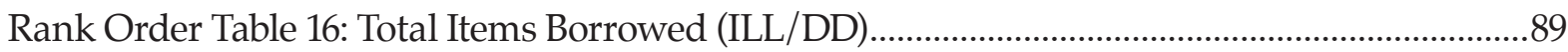

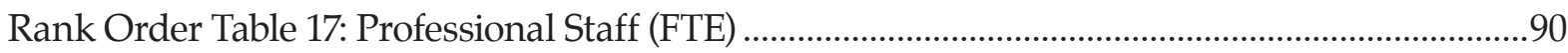

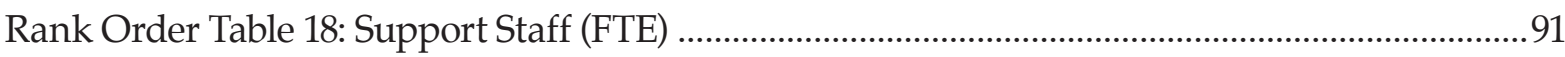

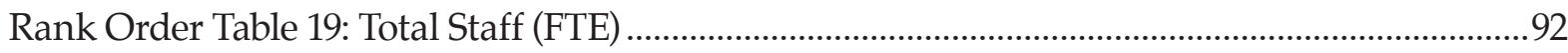

Rank Order Table 20: Expenditures for Electronic Resources .........................................................93

Rank Order Table 21: Electronic Resources as a Percentage of Total Library Materials.................94

Rank Order Table 22: Library Investment Index...............................................................................95

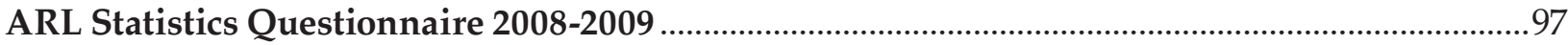

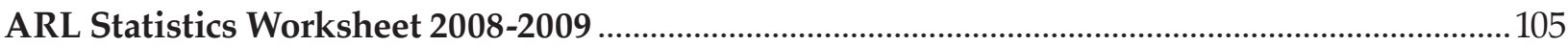

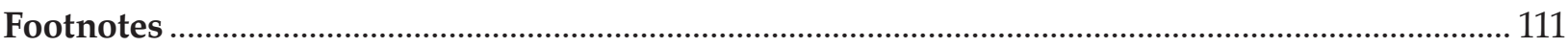

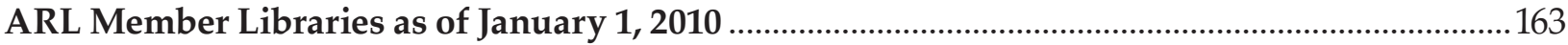

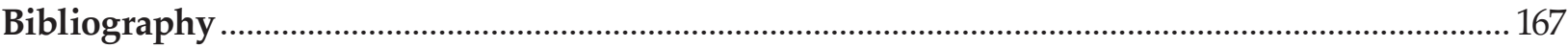




\section{ReSEARCh LibRary TrendS}

ARL Statistics 2008-2009 is the latest in a series of annual publications that describe collections, staffing, expenditures, and service activities for the 124 members of the Association of Research Libraries (ARL). Of these, 114 are university libraries; the remaining 10 are public, governmental, and nonprofit research libraries. ARL member libraries are the largest research libraries in North America, representing $17^{1}$ Canadian and 107 U.S. research institutions. The academic libraries, which comprise about $92 \%$ of the membership, include 15 Canadian and 99 U.S. libraries.

Statistics have been collected and published annually for the members of the Association of Research Libraries since 1961-62, and the data are available through an interactive Web interface. Prior to 1961-62, annual statistics for university libraries were collected by James Gerould, first at the University of Minnesota and later at Princeton University. ${ }^{2}$ These data, covering the years 1907-08 through 1961-62, are now called the Gerould statistics. ${ }^{3}$ The whole data series from 1908, which is available on the ARL website, ${ }^{4}$ represents the oldest and most comprehensive continuing library statistical series in North America.

ARL libraries are a relatively small subset of libraries in North America, but they do account for a large portion of academic library resources in terms of assets, budgets, and the number of users they serve. The total library expenditures of all 124 member libraries in 2008-2009 was slightly more than $\$ 4$ billion; from that, roughly $\$ 3.1$ billion was spent by the 114 university libraries and slightly more than $\$ 940$ million by the nonuniversity libraries. The pie charts below show how the two types of libraries divide these expenditures differently.

University Libraries 2008-2009

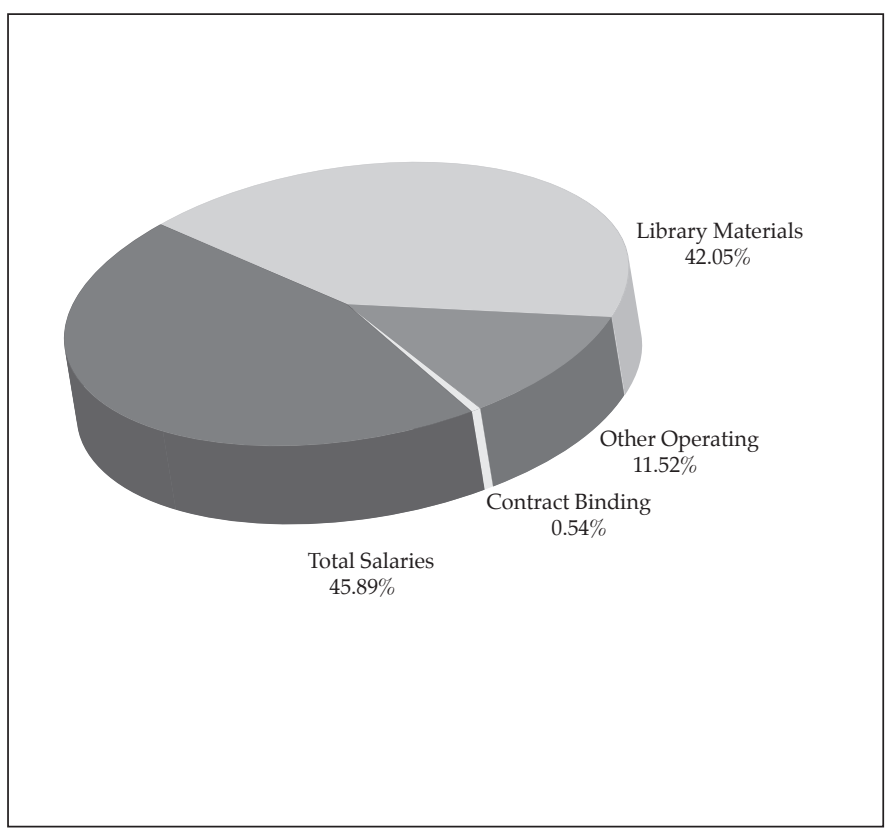

Nonuniversity Libraries 2008-2009

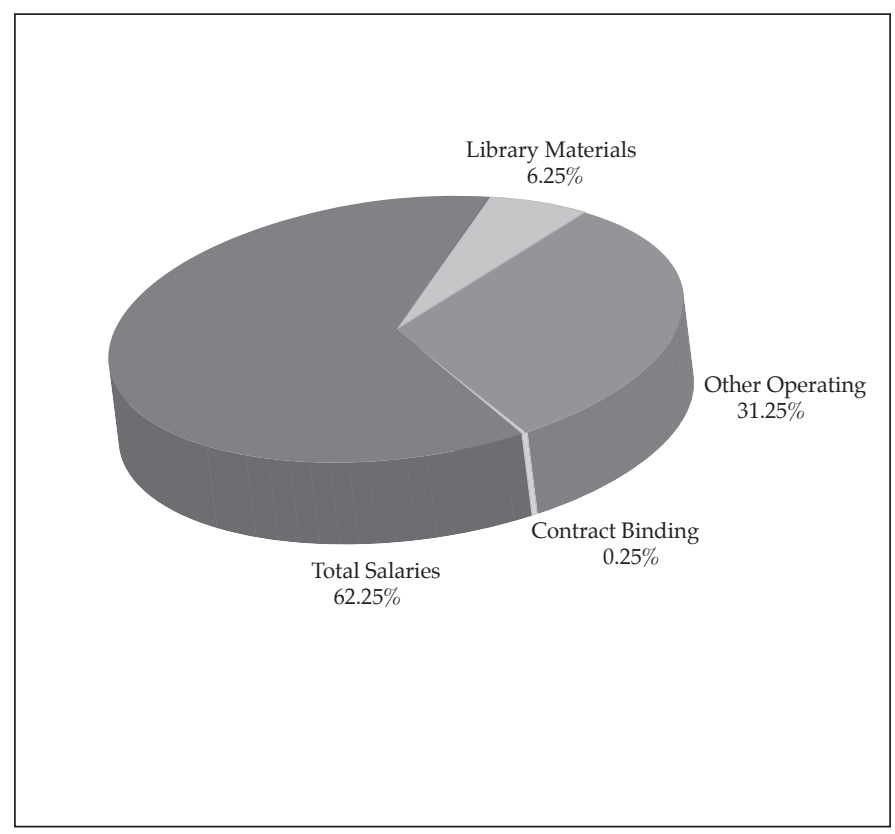

1 CISTI was unable to complete the 2008-2009 ARL Statistics survey due to organizational considerations.

2 Kendon L. Stubbs and Robert E. Molyneux, Research Library Statistics 1907-08 through 1987-88 (Washington, DC: ARL, 1990).

3 Robert E. Molyneux, The Gerould Statistics 1907/08 - 1961/62. (Washington, DC: ARL, 1986), http: / fisher.lib.virginia.edu/gerould/.

4 http://www.arl.org/stats/annualsurveys/arlstats/mrstat.shtml 


\section{Change in Counting Serials}

For the third year in a row libraries have been instructed to count and report serial titles in the ARL Statistics, rather than subscriptions. As a result, the trend line of publishing serial cost per subscription has been discontinued, and eventually a trend line reflecting the new approach to counting serials will emerge as we aggregate annual data on serial titles. This simple shift is very important as it makes the concept of serials more meaningful in the electronic environment.

In the electronic environment, once a library owns or leases a title, the title is often accessible by all users. Therefore, unique titles, rather than subscriptions, is a more authentic descriptive statistic for the scope and content of library collections. A unique title count favors broader coverage. Any duplication of those titles through packages, aggregations, bundles, etc., becomes more a management issue. The new definition asks that serial titles be reported as electronic if available both in print and electronic formats and that they be reported as purchased if available both through purchased and non-purchased arrangements. So, if a serial title appears in both print and electronic form and a library has acquired it through several different providers, it would be counted as one serial title. Training materials have been posted on the ARL website to ensure that there is a well-grounded, shared understanding of the new definitions and counting methodology.

What prompted this change? In earlier years libraries were instructed to report the "total number of subscriptions, not titles, but electronic serials acquired as part of an aggregated package (such as MUSE or Academic's IDEAL) were to be counted by title". Directors and other staff expressed concern that the serials count was problematic because many libraries engage in multiple consortia arrangements. Counting serials purchased through a bevy of consortial agreements could lead to inflated figures because duplicate titles could be held in multiple packages. The Statistics and Assessment Committee determined that a new way of counting serials that focuses on titles would provide better descriptive data reflecting the true scope of the content provided by research libraries. A pilot at various ARL libraries demonstrated the feasibility of the new method.

The shift from counting subscriptions to counting titles further supports ARL's goal of reporting collectable, useful data in the ARL Statistics. Libraries are reporting more serials titles than they ever managed to report by simply counting serial subscriptions. Although the aforementioned positive outcome demonstrates the success of this semantic and methodological shift, all earlier trend lines that were based on serial subscriptions had to be deleted from the publication in order to bring the graphs in line with the new definition and counting methodology. A variety of resources have been developed for libraries to consult as they implement this change, and they are available on the ARL Website at

http://www.arl.org/stats/annualsurveys/arlstats/09statmail.shtml.

In 2007-2008, we changed the categories of serials reported under "serials titles currently received but not purchased." The subcategories are now: (a) consortial, (b) freely accessible, (c) print (and other formats) - exchanges, gifts, etc., and (d) government documents. These categories are more meaningful in the context of "serials titles received," as they emphasize major components of that concept. 


\section{Research Library Trends Tables and GraphS}




\section{TABLE 1}

\section{Service Trends in ARL Libraries, 1991-2009 Median Values for Time-Series Trends}

\begin{tabular}{|c|c|c|c|c|c|c|c|c|c|}
\hline Year & $\begin{array}{r}\text { ILL: } \\
\text { Borrowed }\end{array}$ & $\begin{array}{c}\text { Group } \\
\text { Pres. }\end{array}$ & $\begin{array}{r}\text { Participants } \\
\text { In Pres. }\end{array}$ & $\begin{array}{c}\text { Reference } \\
\text { Trans. }\end{array}$ & $\begin{array}{r}\text { Initial } \\
\text { Circ. }\end{array}$ & $\begin{array}{l}\text { Total } \\
\text { Circ. }\end{array}$ & $\begin{array}{l}\text { Ratio of } \\
\text { Init./Tot. }\end{array}$ & $\begin{array}{l}\text { Total } \\
\text { Staff } \\
\end{array}$ & $\begin{array}{r}\text { Total } \\
\text { Students }\end{array}$ \\
\hline (Libraries) & (103) & $(84)$ & (82) & $(79)$ & (36) & (80) & (34) & (105) & (103) \\
\hline 1991 & 10,397 & 508 & 7,137 & 125,103 & 296,964 & 509,673 & 1.26 & 271 & 18,290 \\
\hline 1992 & 11,362 & 526 & 7,154 & 132,549 & 342,989 & 554,579 & 1.27 & 265 & 18,273 \\
\hline 1993 & 12,489 & 616 & 7,688 & 136,115 & 343,293 & 568,628 & 1.32 & 262 & 18,450 \\
\hline 1994 & 14,007 & 568 & 7,831 & 147,582 & 369,996 & 572,749 & 1.31 & 264 & 18,305 \\
\hline 1995 & 14,472 & 687 & 8,461 & 147,023 & 347,144 & 578,989 & 1.32 & 267 & 18,209 \\
\hline 1996 & 15,278 & 719 & 8,410 & 155,336 & 336,481 & 560,244 & 1.39 & 264 & 18,320 \\
\hline 1997 & 16,264 & 687 & 9,218 & 149,659 & 348,157 & 542,438 & 1.37 & 273 & 18,166 \\
\hline 1998 & 17,656 & 698 & 9,462 & 132,850 & 354,924 & 514,574 & 1.37 & 273 & 18,335 \\
\hline 1999 & 18,942 & 711 & 9,406 & 128,696 & 300,923 & 514,087 & 1.38 & 277 & 18,609 \\
\hline 2000 & 20,475 & 722 & 9,596 & 115,636 & 273,231 & 482,542 & 1.42 & 267 & 18,908 \\
\hline 2001 & 21,902 & 669 & 10,121 & 104,409 & 265,195 & 467,277 & 1.48 & 269 & 19,102 \\
\hline 2002 & 21,339 & 776 & 11,350 & 95,910 & 251,146 & 462,223 & 1.51 & 279 & 19,925 \\
\hline 2003 & 22,146 & 806 & 12,516 & 89,150 & 248,689 & 479,733 & 1.57 & 277 & 21,132 \\
\hline 2004 & 25,737 & 757 & 12,864 & 84,546 & 261,526 & 496,369 & 1.60 & 273 & 21,562 \\
\hline 2005 & 25,729 & 803 & 13,782 & 65,168 & 250,971 & 473,216 & 1.58 & 267 & 22,047 \\
\hline 2006 & 27,412 & 833 & 13,051 & 67,697 & 267,213 & 466,403 & 1.52 & 267 & 22,618 \\
\hline 2007 & 26,813 & 830 & 14,417 & 61,703 & 222,037 & 456,597 & 1.59 & 266 & 22,874 \\
\hline 2008 & 27,822 & 803 & 15,480 & 58,763 & 221,144 & 429,626 & 1.59 & 260 & 22,762 \\
\hline 2009 & 28,187 & 877 & 14,958 & 55,416 & 218,191 & 414,482 & 1.64 & 249 & 23,303 \\
\hline $\begin{array}{l}\text { Average annual } \\
\% \text { change }\end{array}$ & $5.7 \%$ & $3.1 \%$ & $4.2 \%$ & $-4.4 \%$ & $-1.7 \%$ & $-1.1 \%$ & $1.5 \%$ & $-0.5 \%$ & $1.4 \%$ \\
\hline
\end{tabular}

Table 1 presents data about select public service activities such as circulation (initial and total), reference transactions, library instruction (group presentations and participants in these presentations), and interlibrary borrowing and lending. As seen in table 1, for the first time in three years, the median of group presentations increased. However, the number of participants decreased with a median of 14,958 for the typical research library. Perhaps what is of most interest is that by 2009 there were more than double the participants compared to 1991, while there is only a $27 \%$ difference in the number of students between 1991 and 2009 (see Graph 1). Graph 1 shows that, cumulatively, total staff decreased by 8\% in 2009, which is double the cumulative decrease reported in 2008. Even with continued decreases in total staff, the median of group presentations increased in 2009. 


\section{GRAPH 1}

Service Trends in ARL Libraries, 1991-2009

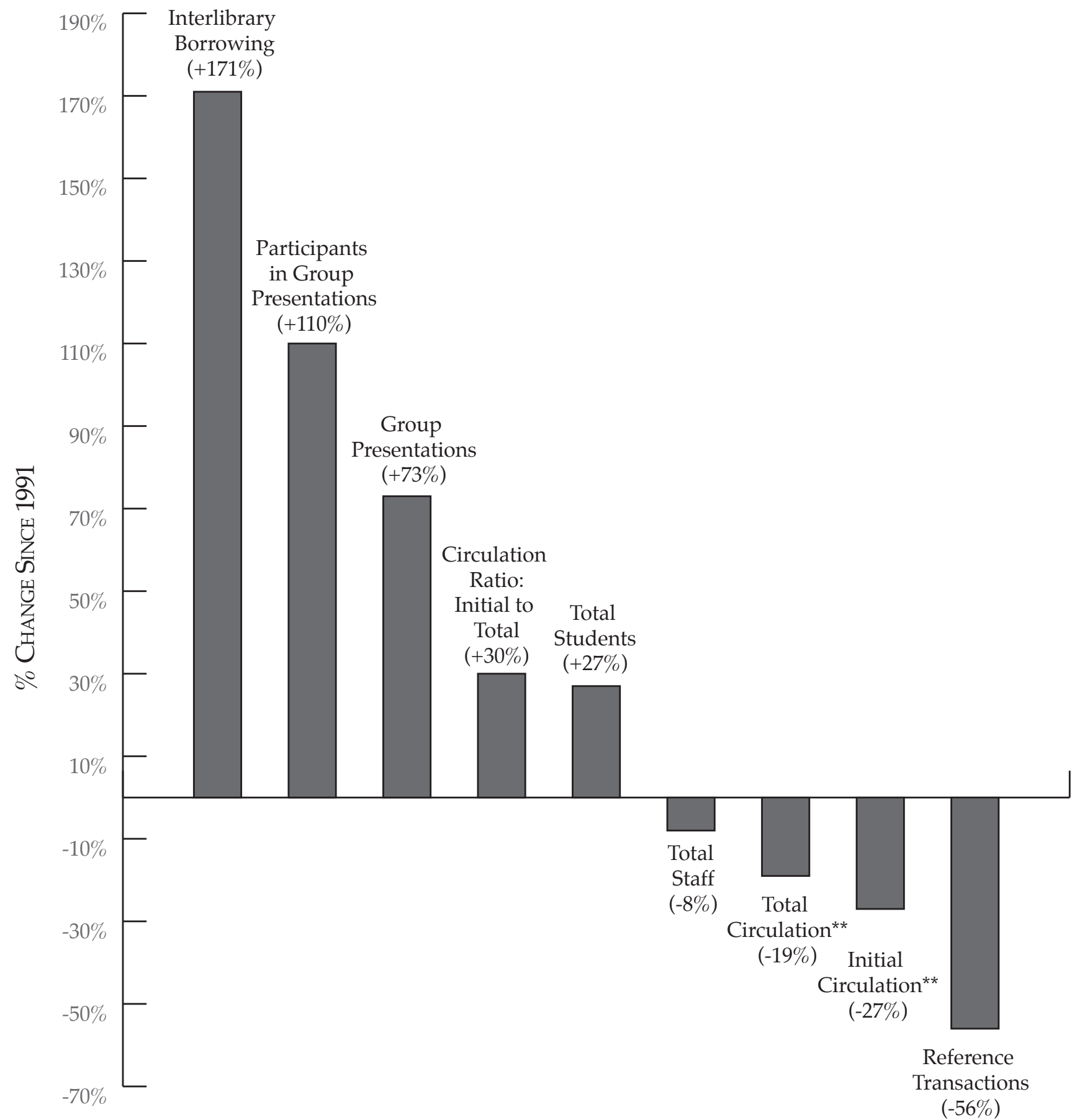

** Total Circulation includes Initial and Renewal but excludes Reserve Circulation.

Source: ARL Statistics 2008-2009, Association of Research Libraries, Washington, DC 


\section{TABLE 2}

Monograph and Serial Expenditures in ARL Libraries, 1986-2009 Median Values for Time-Series Trends

\begin{tabular}{|c|c|c|c|c|}
\hline Year & $\begin{array}{r}\text { Serial } \\
\text { Expenditures } \\
\end{array}$ & $\begin{array}{r}\text { Monograph Unit } \\
\text { Cost } \\
\end{array}$ & $\begin{array}{r}\text { Monograph } \\
\text { Expenditures }\end{array}$ & $\begin{array}{r}\text { Monographs } \\
\text { Purchased } \\
\end{array}$ \\
\hline (Libraries) & (100) & (57) & (97) & (58) \\
\hline 1986 & $\$ 1,496,775$ & $\$ 29.28$ & $\$ 1,120,645$ & 32,679 \\
\hline 1987 & $\$ 1,769,960$ & $\$ 31.76$ & $\$ 1,064,484$ & 26,240 \\
\hline 1988 & $\$ 1,947,559$ & $\$ 36.03$ & $\$ 1,141,226$ & 25,238 \\
\hline 1989 & $\$ 2,113,976$ & $\$ 38.44$ & $\$ 1,106,073$ & 27,082 \\
\hline 1990 & $\$ 2,296,910$ & $\$ 41.44$ & $\$ 1,330,747$ & 27,546 \\
\hline 1991 & $\$ 2,548,687$ & $\$ 42.60$ & $\$ 1,400,738$ & 27,843 \\
\hline 1992 & $\$ 2,620,832$ & $\$ 44.14$ & $\$ 1,353,865$ & 26,947 \\
\hline 1993 & $\$ 2,918,569$ & $\$ 43.74$ & $\$ 1,295,807$ & 25,188 \\
\hline 1994 & $\$ 2,912,495$ & $\$ 44.72$ & $\$ 1,309,807$ & 25,341 \\
\hline 1995 & $\$ 3,131,033$ & $\$ 45.27$ & $\$ 1,365,575$ & 25,707 \\
\hline 1996 & $\$ 3,389,118$ & $\$ 46.73$ & $\$ 1,444,015$ & 25,911 \\
\hline 1997 & $\$ 3,642,541$ & $\$ 46.42$ & $\$ 1,460,234$ & 28,576 \\
\hline 1998 & $\$ 3,816,497$ & $\$ 47.59$ & $\$ 1,486,764$ & 24,447 \\
\hline 1999 & $\$ 4,095,934$ & $\$ 47.78$ & $\$ 1,506,651$ & 25,173 \\
\hline 2000 & $\$ 4,430,812$ & $\$ 47.87$ & $\$ 1,657,349$ & 28,024 \\
\hline 2001 & $\$ 4,660,349$ & $\$ 48.48$ & $\$ 1,864,023$ & 30,494 \\
\hline 2002 & $\$ 4,939,225$ & $\$ 50.61$ & $\$ 1,812,826$ & 31,578 \\
\hline 2003 & $\$ 5,392,007$ & $\$ 52.83$ & $\$ 1,873,157$ & 33,208 \\
\hline 2004 & $\$ 5,580,157$ & $\$ 51.36$ & $\$ 1,839,412$ & 30,155 \\
\hline 2005 & $\$ 5,962,446$ & $\$ 53.57$ & $\$ 1,784,841$ & 30,546 \\
\hline 2006 & $\$ 6,307,292$ & $\$ 52.04$ & $\$ 2,044,272$ & 33,145 \\
\hline 2007 & $\$ 6,587,241$ & $\$ 54.17$ & $\$ 2,097,078$ & 36,462 \\
\hline 2008 & $\$ 7,097,140$ & $\$ 55.41$ & $\$ 2,084,991$ & 32,745 \\
\hline 2009 & $\$ 7,193,291$ & $\$ 54.82$ & $\$ 1,981,284$ & 34,594 \\
\hline $\begin{array}{l}\text { Average annual } \\
\% \text { change }\end{array}$ & $7.4 \%$ & $2.9 \%$ & $2.6 \%$ & $0.3 \%$ \\
\hline
\end{tabular}

Graph 2 displays the skyrocketing expenditures for serials (in comparison to monograph expenditures). Note that Table and Graph 2 were revised and do not include Serial Unit Cost or Serial Subscriptions anymore because starting in 20062007 we are counting serial titles. 


\section{GRAPH 2}

\section{Monograph and Serial Expenditures in ARL Libraries, 1986-2009*}

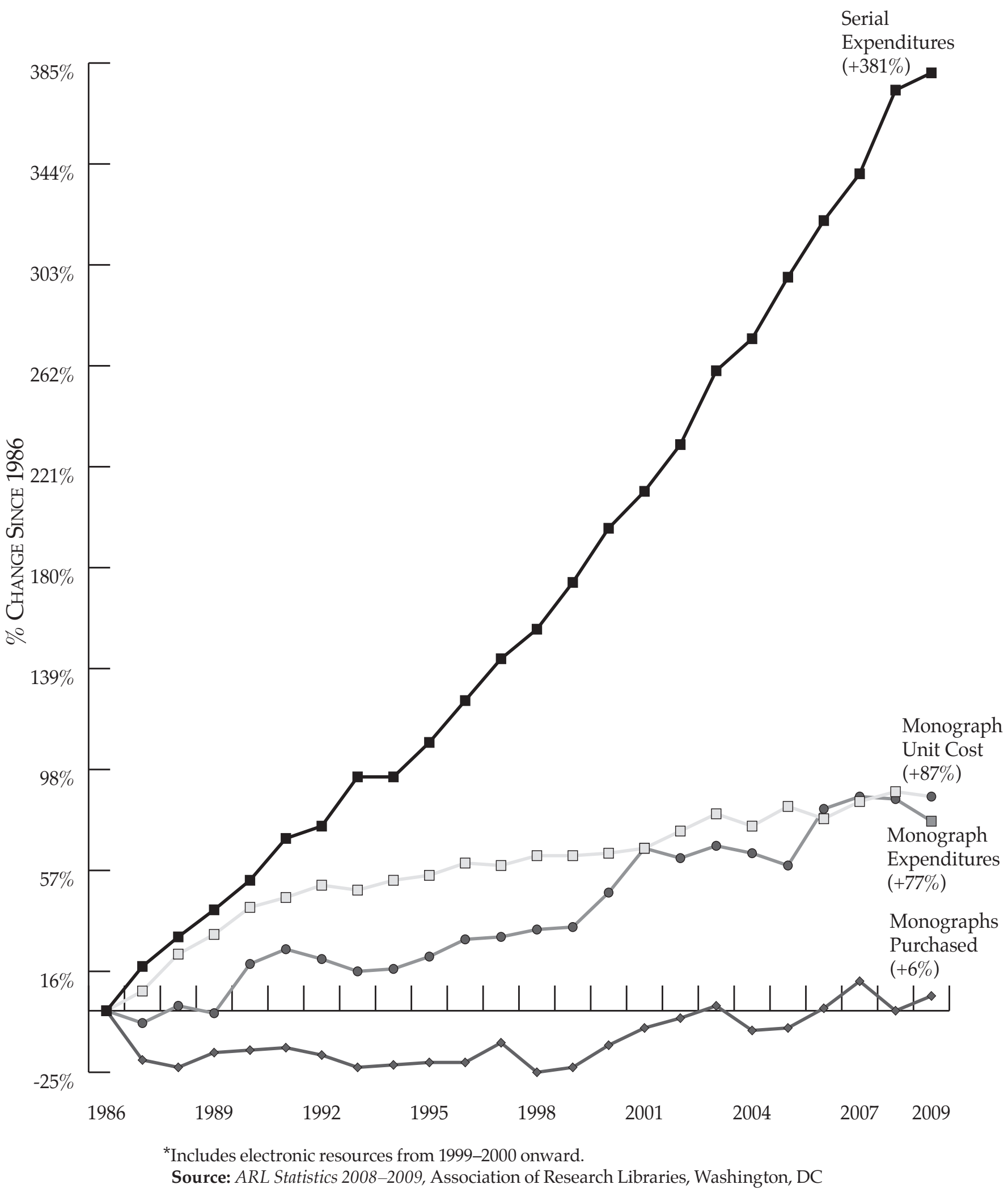


TABLE 3

\section{Supply AND Demand in ARL LibRaRies, 1986-2009 Median Values for Time-Series Trends}

\begin{tabular}{|c|c|c|c|c|c|c|}
\hline Year & $\begin{array}{r}\text { ILL: } \\
\text { Borrowed }\end{array}$ & $\begin{array}{r}\text { ILL: } \\
\text { Loaned }\end{array}$ & $\begin{array}{l}\text { Graduate } \\
\text { Students }\end{array}$ & $\begin{array}{r}\text { Teaching } \\
\text { Faculty }\end{array}$ & $\begin{array}{r}\text { Total } \\
\text { Students }\end{array}$ & $\begin{array}{r}\text { Monographs } \\
\text { Purchased }\end{array}$ \\
\hline (Libraries) & (103) & (103) & (104) & (101) & (103) & (58) \\
\hline 1986 & 7,047 & 16,092 & 2,327 & 1,124 & 16,684 & 32,679 \\
\hline 1987 & 7,387 & 16,318 & 3,078 & 1,195 & 17,029 & 26,240 \\
\hline 1988 & 7,881 & 17,476 & 3,251 & 1,222 & 17,485 & 25,238 \\
\hline 1989 & 8,547 & 19,638 & 3,312 & 1,285 & 17,866 & 27,082 \\
\hline 1990 & 9,595 & 20,837 & 3,314 & 1,278 & 17,745 & 27,546 \\
\hline 1991 & 10,397 & 23,285 & 3,310 & 1,295 & 18,290 & 27,843 \\
\hline 1992 & 11,362 & 22,514 & 3,539 & 1,356 & 18,273 & 26,947 \\
\hline 1993 & 12,489 & 22,740 & 3,745 & 1,281 & 18,450 & 25,188 \\
\hline 1994 & 14,007 & 24,039 & 3,794 & 1,289 & 18,305 & 25,341 \\
\hline 1995 & 14,472 & 24,864 & 3,914 & 1,308 & 18,209 & 25,707 \\
\hline 1996 & 15,278 & 25,720 & 3,904 & 1,251 & 18,320 & 25,911 \\
\hline 1997 & 16,264 & 25,463 & 3,942 & 1,263 & 18,166 & 28,576 \\
\hline 1998 & 17,656 & 27,223 & 3,880 & 1,247 & 18,335 & 24,447 \\
\hline 1999 & 18,942 & 26,837 & 3,933 & 1,255 & 18,609 & 25,173 \\
\hline 2000 & 20,475 & 27,044 & 3,844 & 1,239 & 18,908 & 28,024 \\
\hline 2001 & 21,902 & 28,950 & 4,159 & 1,279 & 19,102 & 30,494 \\
\hline 2002 & 21,339 & 29,021 & 4,067 & 1,251 & 19,925 & 31,578 \\
\hline 2003 & 22,146 & 33,421 & 4,167 & 1,268 & 21,132 & 33,208 \\
\hline 2004 & 25,737 & 33,934 & 4,461 & 1,369 & 21,562 & 30,155 \\
\hline 2005 & 25,729 & 36,325 & 4,595 & 1,355 & 22,047 & 30,546 \\
\hline 2006 & 27,412 & 36,035 & 4,647 & 1,388 & 22,618 & 33,145 \\
\hline 2007 & 26,813 & 35,838 & 4,709 & 1,451 & 22,874 & 36,462 \\
\hline 2008 & 27,822 & 36,383 & 4,905 & 1,490 & 22,762 & 32,745 \\
\hline 2009 & 28,187 & 35,589 & 5,108 & 1,425 & 23,303 & 34,594 \\
\hline $\begin{array}{l}\text { Average annual } \\
\% \text { change }\end{array}$ & $6.5 \%$ & $3.7 \%$ & $3.6 \%$ & $1.1 \%$ & $1.5 \%$ & $0.3 \%$ \\
\hline
\end{tabular}

Interlibrary borrowing, which showed a marked growth in the last decade, continued to increase in 2008-2009. However, interlibrary lending decreased slightly over 2008 levels. Despite this slight downward trend in interlibrary lending in 2009, libraries continued to lend more than they borrowed overall. 


\section{GRAPH 3}

\section{Supply and Demand in ARL Libraries, 1986-2009*}

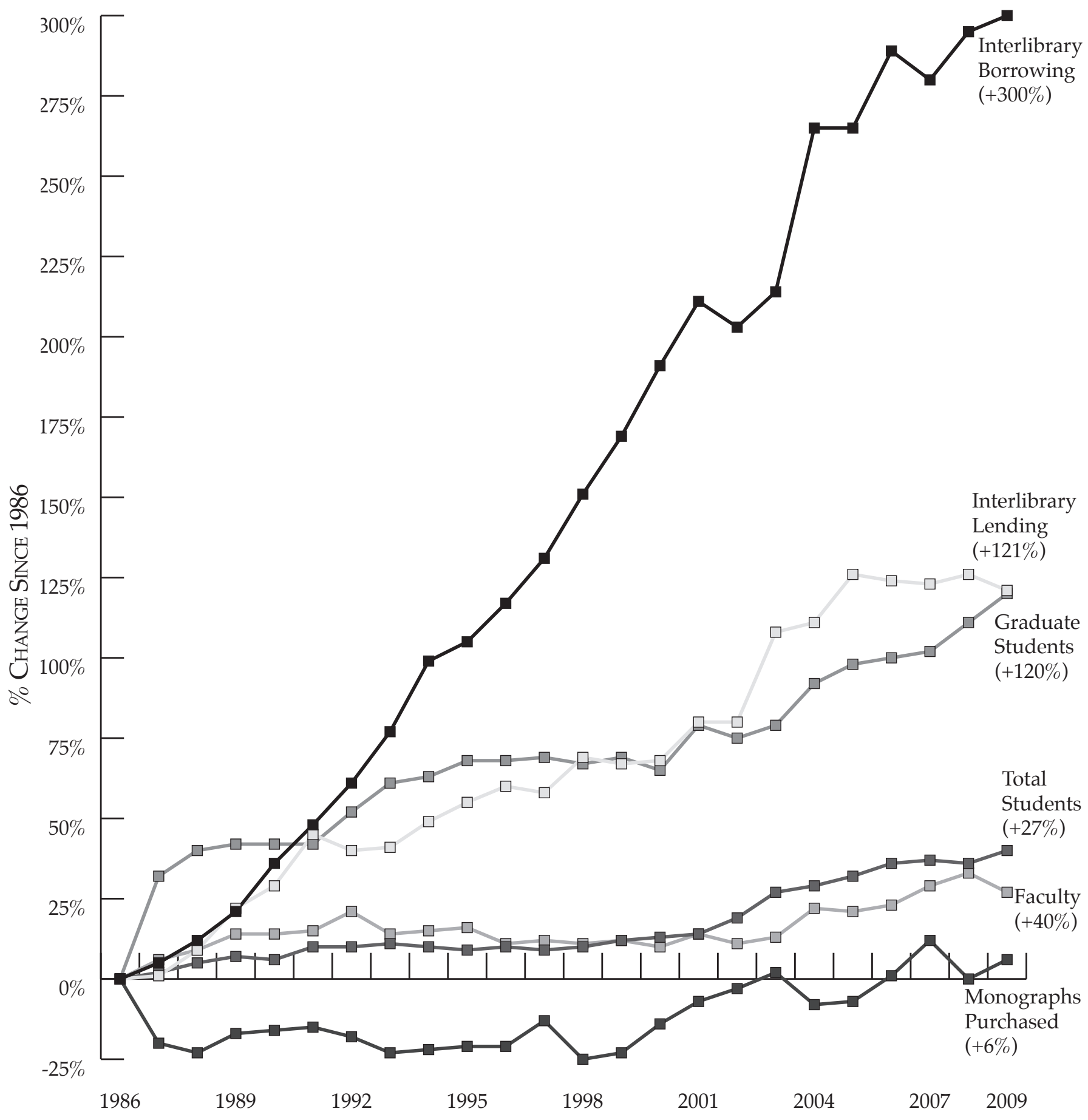

*Serial and monograph data includes electronic resources from 1999-2000 onward.

Source: ARL Statistics 2008-2009, Association of Research Libraries, Washington, DC 


\section{TABLE 4}

\section{EXPENDiture Trends in ARL LibraRies, 1986-2009 Median Values for Time-Series Trends-Unadjusted dollar figures}

\begin{tabular}{|c|c|c|c|c|c|c|c|}
\hline Year & $\begin{array}{r}\text { Library } \\
\text { Materials }\end{array}$ & Serial Exp. & $\begin{array}{r}\text { Monograph } \\
\text { Exp. }\end{array}$ & Total Salary & $\begin{array}{r}\text { Operating } \\
\text { Exp. }\end{array}$ & Total & CPI \\
\hline (Libraries) & (105) & (100) & (97) & (104) & (104) & (105) & \\
\hline 1986 & $2,705,297$ & $1,496,775$ & $1,120,645$ & $4,011,436$ & $1,111,914$ & $8,361,092$ & 109.6 \\
\hline 1987 & $3,058,479$ & $1,769,960$ & $1,064,484$ & $4,361,646$ & $1,180,167$ & $8,990,001$ & 113.6 \\
\hline 1988 & $3,369,896$ & $1,947,559$ & $1,141,226$ & $4,618,335$ & $1,198,674$ & $9,557,623$ & 118.3 \\
\hline 1989 & $3,577,405$ & $2,113,976$ & $1,106,073$ & $5,236,292$ & $1,364,558$ & $10,183,315$ & 124.0 \\
\hline 1990 & $3,903,358$ & $2,296,910$ & $1,330,747$ & $5,469,333$ & $1,386,618$ & $11,241,022$ & 130.7 \\
\hline 1991 & $4,064,344$ & $2,548,687$ & $1,400,738$ & $5,885,814$ & $1,445,735$ & $11,990,794$ & 136.2 \\
\hline 1992 & $4,156,510$ & $2,620,832$ & $1,353,865$ & $6,050,222$ & $1,390,245$ & $12,249,150$ & 140.3 \\
\hline 1993 & $4,316,674$ & $2,918,569$ & $1,295,807$ & $5,962,470$ & $1,561,122$ & $12,265,696$ & 144.5 \\
\hline 1994 & $4,572,276$ & $2,912,495$ & $1,309,807$ & $6,047,803$ & $1,676,701$ & $12,767,348$ & 148.2 \\
\hline 1995 & $4,715,203$ & $3,131,033$ & $1,365,575$ & $6,312,770$ & $1,853,586$ & $13,171,893$ & 152.4 \\
\hline 1996 & $5,126,482$ & $3,389,118$ & $1,444,015$ & $6,664,021$ & $1,997,233$ & $13,870,378$ & 156.9 \\
\hline 1997 & $5,562,742$ & $3,642,541$ & $1,460,234$ & $6,893,582$ & $2,039,957$ & $14,526,674$ & 160.5 \\
\hline 1998 & $5,795,223$ & $3,816,497$ & $1,486,764$ & $7,163,979$ & $2,072,903$ & $15,329,371$ & 163.0 \\
\hline 1999 & $6,232,365$ & $4,095,934$ & $1,506,651$ & $7,476,532$ & $2,069,887$ & $16,737,261$ & 166.6 \\
\hline 2000 & $6,744,281$ & $4,430,812$ & $1,657,349$ & $7,811,403$ & $1,991,852$ & $17,221,441$ & 172.2 \\
\hline 2001 & $7,322,507$ & $4,660,349$ & $1,864,023$ & $8,106,666$ & $2,280,493$ & $17,620,048$ & 177.5 \\
\hline 2002 & $7,599,249$ & $4,939,225$ & $1,812,826$ & $8,488,255$ & $2,136,616$ & $18,456,038$ & 180.1 \\
\hline 2003 & $8,273,171$ & $5,392,007$ & $1,873,157$ & $8,813,191$ & $2,073,913$ & $19,030,188$ & 183.9 \\
\hline 2004 & $8,286,431$ & $5,580,157$ & $1,839,412$ & $9,015,741$ & $2,274,878$ & $19,953,776$ & 189.4 \\
\hline 2005 & $8,801,962$ & $5,962,446$ & $1,784,841$ & $9,268,364$ & $2,243,592$ & $20,663,012$ & 195.4 \\
\hline 2006 & $9,174,734$ & $6,307,292$ & $2,044,272$ & $9,647,695$ & $2,540,171$ & $21,694,210$ & 203.5 \\
\hline 2007 & $9,600,793$ & $6,587,241$ & $2,097,078$ & $9,940,406$ & $2,549,139$ & $22,810,227$ & 208.3 \\
\hline 2008 & $10,454,644$ & $7,097,140$ & $2,084,991$ & $10,302,966$ & $2,649,899$ & $24,084,535^{*}$ & 219.9 \\
\hline 2009 & $10,185,101$ & $7,193,291$ & $1,981,284$ & $10,462,624$ & $2,530,251$ & $23,622,720$ & 215.4 \\
\hline $\begin{array}{l}\text { Average annual } \\
\% \text { change }\end{array}$ & $6.2 \%$ & $7.4 \%$ & $2.6 \%$ & $4.5 \%$ & $3.8 \%$ & $4.8 \%$ & $3.0 \%$ \\
\hline
\end{tabular}

*2007-2008 publication incorrectly reported 24,804,535.

Operating expenditures, including many automation expenditures, have doubled since 1986, but have remained at relatively stable levels of investment since 2008. The total salary expenditures median remained basically the same since last year (the overall annual average rate since 1986 is 4.5\%). Monograph expenditures continue to increase, but have in 2008-2009 increased at a lower rate than the consumer price index (CPI). Total Library Expenditures decreased in 2009 as compared to 2008, even as serial expenditures continued to rise. The annual consumer price index, included in Graph 4, provides a comparative reference for the increases in library expenditures. 


\section{GRAPH 4}

\section{EXPENDiture Trends in ARL LibRaRies, 1986-2009}

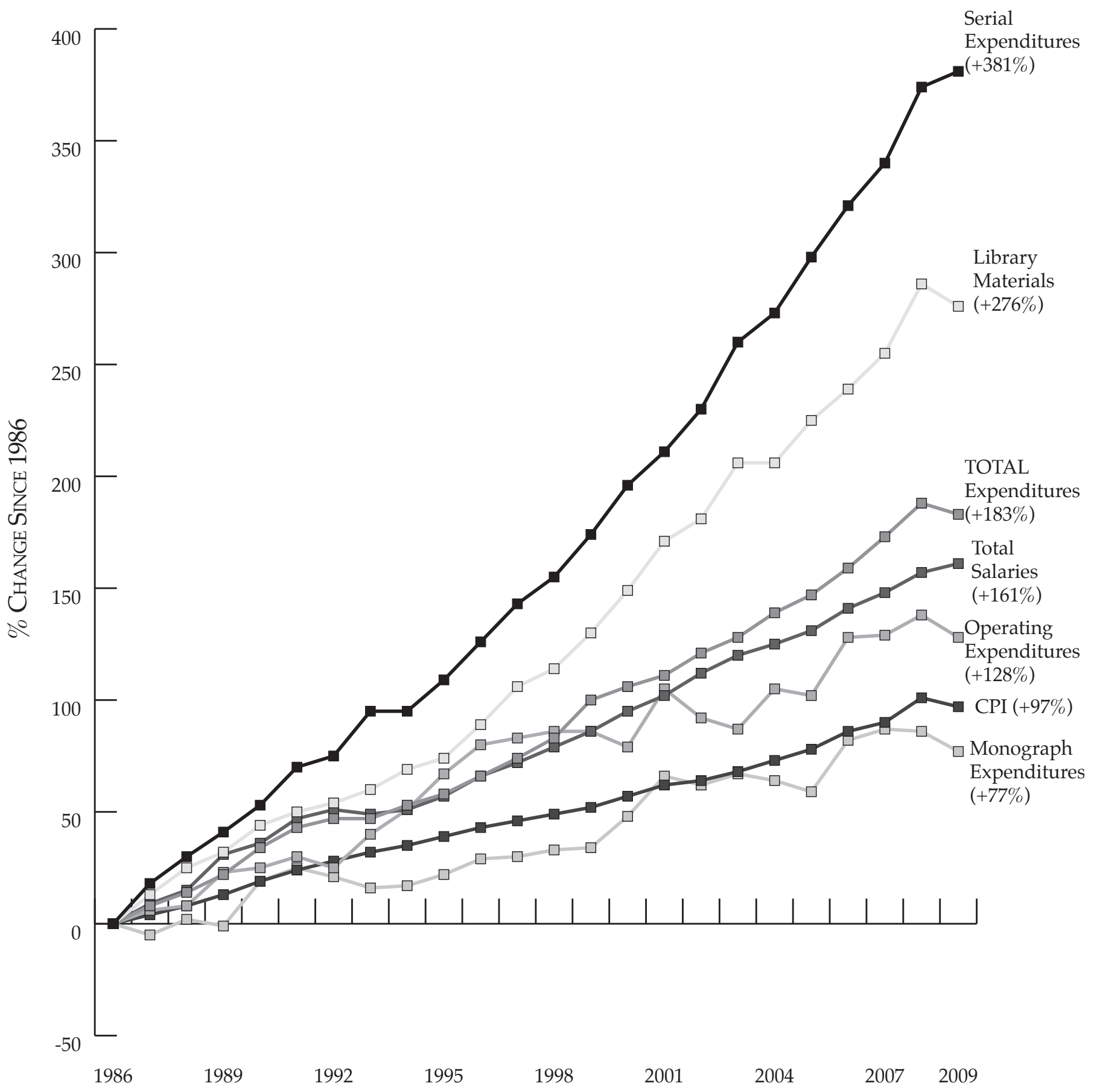

Source: ARL Statistics 2008-2009, Association of Research Libraries, Washington, DC 
TABLE 5

\section{Resources Per Student in ARL University Libraries, 1986-2009 Median of Ratio Values for Time-Series Trends}

\begin{tabular}{|c|c|c|c|c|c|}
\hline Year & $\begin{array}{r}\text { ILL: } \\
\text { Borrowed }\end{array}$ & $\begin{array}{r}\text { Volumes } \\
\text { Held }\end{array}$ & $\begin{array}{r}\text { Volumes } \\
\text { Added (Gross) }\end{array}$ & Total Staff & $\begin{array}{r}\text { Monographs } \\
\text { Purchased }\end{array}$ \\
\hline (Libraries) & (102) & (103) & (103) & (103) & (57) \\
\hline 1986 & 0.42 & 123.18 & 4.17 & 0.0159 & 2.20 \\
\hline 1987 & 0.44 & 125.31 & 3.92 & 0.0164 & 1.88 \\
\hline 1988 & 0.44 & 128.27 & 3.82 & 0.0161 & 1.66 \\
\hline 1989 & 0.50 & 130.04 & 3.84 & 0.0162 & 1.77 \\
\hline 1990 & 0.55 & 133.51 & 3.86 & 0.0155 & 1.83 \\
\hline 1991 & 0.63 & 137.11 & 4.04 & 0.0154 & 1.86 \\
\hline 1992 & 0.62 & 137.68 & 3.95 & 0.0153 & 1.58 \\
\hline 1993 & 0.68 & 146.02 & 3.76 & 0.0152 & 1.49 \\
\hline 1994 & 0.74 & 151.20 & 4.07 & 0.0152 & 1.55 \\
\hline 1995 & 0.80 & 154.00 & 4.37 & 0.0147 & 1.59 \\
\hline 1996 & 0.89 & 158.10 & 3.78 & 0.0145 & 1.48 \\
\hline 1997 & 0.89 & 159.40 & 4.03 & 0.0142 & 1.52 \\
\hline 1998 & 0.97 & 159.24 & 4.06 & 0.0143 & 1.44 \\
\hline 1999 & 1.05 & 159.37 & 4.00 & 0.0142 & 1.42 \\
\hline 2000 & 1.13 & 157.74 & 3.82 & 0.0139 & 1.63 \\
\hline 2001 & 1.16 & 155.71 & 3.89 & 0.0136 & 1.69 \\
\hline 2002 & 1.14 & 155.04 & 3.87 & 0.0131 & 1.54 \\
\hline 2003 & 1.14 & 156.41 & 3.52 & 0.0125 & 1.47 \\
\hline 2004 & 1.20 & 151.49 & 3.45 & 0.0120 & 1.53 \\
\hline 2005 & 1.22 & 157.93 & 3.30 & 0.0118 & 1.42 \\
\hline 2006 & 1.22 & 160.05 & 3.26 & 0.0117 & 1.64 \\
\hline 2007 & 1.13 & 159.49 & 3.49 & 0.0114 & 1.54 \\
\hline 2008 & 1.19 & 162.87 & 3.22 & 0.0110 & 1.40 \\
\hline 2009 & 1.24 & 165.49 & 3.59 & 0.0110 & 1.56 \\
\hline $\begin{array}{l}\text { Average annual } \\
\% \text { change }\end{array}$ & $5.0 \%$ & $1.4 \%$ & $-0.7 \%$ & $-1.7 \%$ & $-1.5 \%$ \\
\hline
\end{tabular}

In 2008-2009, ARL libraries were acquiring 29\% fewer monographs per student than purchased in 1986. Libraries reported fewer staff per student in 2009 as compared to 1986: in 2009, there was a median number of 11 total staff per 1,000 students, compared to the 1986 figure of 16 per 1,000. However, libraries have not let this trend prevent them from meeting the information needs of their students: interlibrary borrowing, volumes held, volumes added gross, and monographs purchased all increased over the respective 2008 figures. 


\section{GRAPH 5}

\section{Resources per Student in ARL University Libraries, 1986-2009}

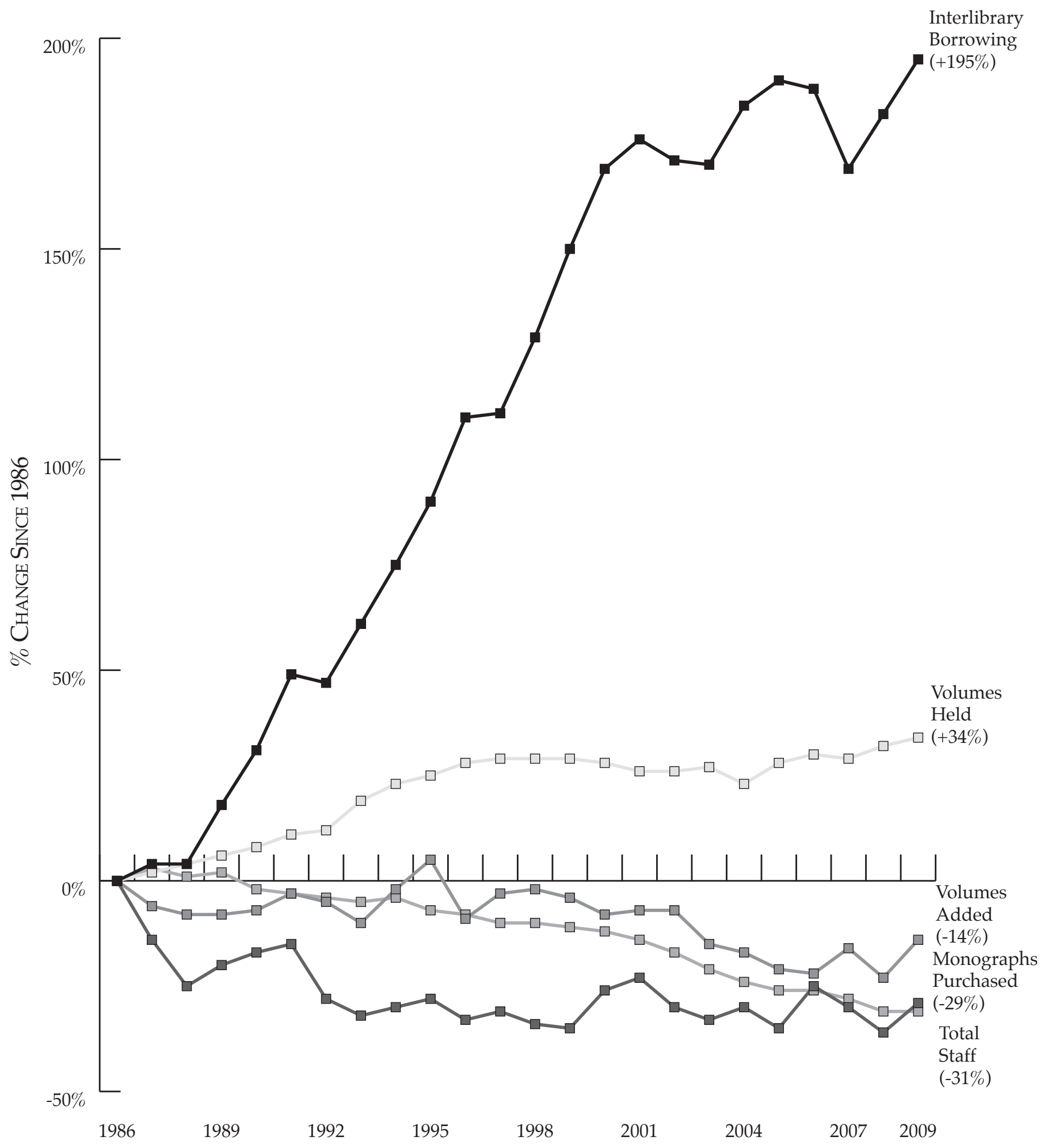

Source: ARL Statistics 2008-2009, Association of Research Libraries, Washington, DC 


\section{TABLE 6}

\section{Electronic Resources Expenditures in ARL University Libraries, 2008-2009}

\section{Sum Number Reporting}

Expenditures for Computer Files (one-time/

monographic purchase)

Expenditures for Electronic Serials

Expenditures for Bibliographic Utilities, Networks, etc.

(Library)

Expenditures for Bibliographic Utilities, Networks, etc.

(External)

Expenditures for Hardware and Software

Expenditures for Document Delivery/Interlibrary Loan
$69,148,203$

110

$637,458,376$

112

$24,325,261$

103

$21,695,047$

92

$77,239,838$

111

$16,327,690$

Table 6 contains data on several items that previously had been collected only in the ARL Supplementary Statistics. These data are especially useful because they reflect monies spent on all electronic serials, while the ARL Statistics categories of "serials purchased" and "serials expenditures" include only those journals that provide full-text electronic versions to their subscribers. The Expenditures for Electronic Serials time series may be viewed as an alternative to the Serials Purchased series, both figures reflecting in their own way the influence the electronic serial is gaining in the modern research library.

Not only have electronic materials expenditures grown sharply in the past decade, they have grown at a rate far exceeding that of library materials expenditures overall. As shown in Graph 6, in every year of the last decade electronic materials expenditures have grown sharply, anywhere between two and ten times faster than materials expenditures have.

The average ARL university library now spends 56\% of its materials budget on electronic materials (Tables 7A and 7B), and 82 ARL libraries report that they spent more than $50 \%$ of their materials budget on electronic materials (see Rank Order Table 21). 


\section{GRAPH 6}

\section{Electronic Resources vs. Total Materials Expenditures, 1993-2009 Yearly Increases in Average Expenditures}

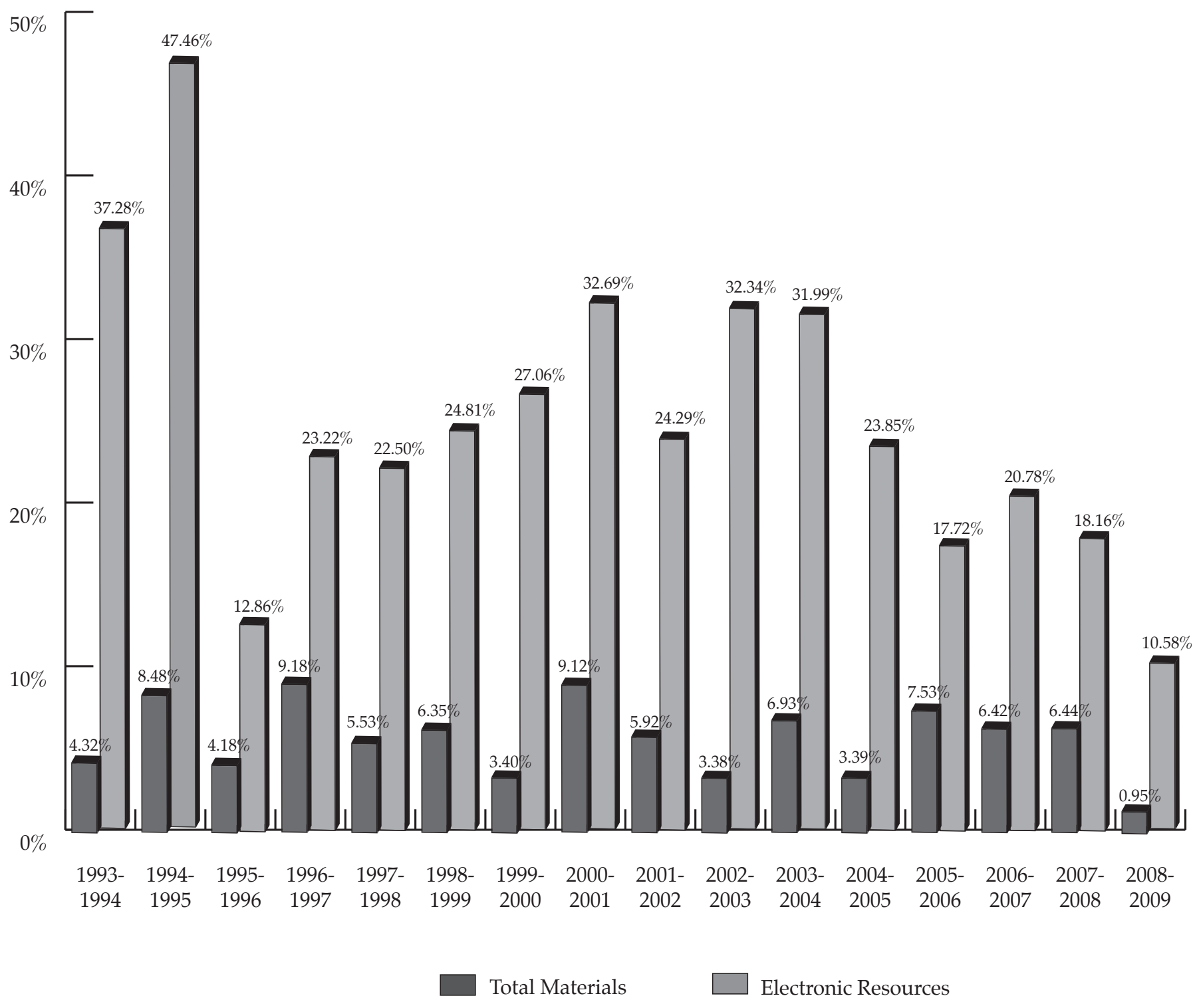




\section{TABLE 7A}

\section{Electronic Resources and Materials Expenditures in ARL University Libraries, 1992-2002}

\begin{tabular}{|c|c|c|c|c|c|c|c|c|c|c|}
\hline & 1992-1993 & 1993-1994 & 1994-1995 & 1995-1996 & 1996-1997 & 1997-1998 & 1998-1999 & 1999-2000 & 2000-2001 & 2001-2002 \\
\hline \multicolumn{11}{|c|}{ a. Computer File Expenditures (monographic/onetime) } \\
\hline Total & $14,147,625$ & $20,132,553$ & $22,030,727$ & $24,639,822$ & $8,013,055$ & $11,189,103$ & $10,848,219$ & $14,727,984$ & $15,297,096$ & $16,748,194$ \\
\hline Average & 172,532 & 236,854 & 247,536 & 262,126 & 87,098 & 122,957 & 121,890 & 161,846 & 159,345 & 167,482 \\
\hline Median & 148,158 & 212,936 & 217,988 & 219,178 & 47,932 & 52,311 & 54,024 & 98,657 & 72,070 & 82,566 \\
\hline $\mathbf{N}$ & & 85 & 89 & 94 & 92 & 91 & 89 & 91 & 96 & 100 \\
\hline \multicolumn{11}{|c|}{ b. Electronic Serial Expenditures } \\
\hline Total & N/A & N/A & $11,847,577$ & $15,170,971$ & $40,956,696$ & $49,497,141$ & $67,124,554$ & $84,343,868$ & $117,415,618$ & $154,418,679$ \\
\hline Average & $\mathrm{N} / \mathrm{A}$ & $\mathrm{N} / \mathrm{A}$ & 188,057 & 194,500 & 401,536 & 494,971 & 639,281 & 818,873 & $1,118,244$ & $1,429,803$ \\
\hline Median & $\mathrm{N} / \mathrm{A}$ & $\mathrm{N} / \mathrm{A}$ & 156,754 & 172,805 & 355,922 & 426,722 & 571,790 & 736,317 & 992,067 & $1,272,965$ \\
\hline $\mathbf{N}$ & & & 63 & 78 & 102 & 100 & 105 & 103 & 105 & 108 \\
\hline \multicolumn{11}{|c|}{ c. Total Electronic Resources (Total $\mathrm{a}+\mathrm{b}$ ) } \\
\hline Total & $14,147,625$ & $20,132,553$ & $33,878,304$ & $39,810,793$ & $50,512,984$ & $60,686,244$ & $77,972,773$ & $99,071,852$ & $132,712,714$ & $171,166,873$ \\
\hline Average & 172,532 & 236,854 & 349,261 & 394,166 & 485,702 & 594,963 & 742,598 & 943,541 & $1,252,007$ & $1,556,062$ \\
\hline Median & 148,158 & 212,936 & 278,404 & 332,128 & 420,741 & 495,011 & 645,495 & 931,210 & $1,129,298$ & $1,377,874$ \\
\hline $\mathbf{N}$ & 82 & 85 & 97 & 101 & 104 & 102 & 105 & 105 & 106 & 110 \\
\hline \multicolumn{11}{|c|}{ Total Library Materials Expenditures* } \\
\hline Total & $393,271,073$ & $425,287,651$ & $526,496,347$ & $571,145,986$ & $642,123,715$ & $664,600,663$ & $727,623,160$ & $773,321,519$ & $828,778,808$ & $910,930,849$ \\
\hline Average & $4,795,989$ & $5,003,384$ & $5,427,797$ & $5,654,911$ & $6,174,266$ & $6,515,692$ & $6,929,744$ & $7,364,967$ & $7,818,668$ & $8,281,189$ \\
\hline Median & $4,242,887$ & $4,527,122$ & $4,714,384$ & $4,975,353$ & $5,529,606$ & $5,643,070$ & $5,991,177$ & $6,545,146$ & $7,028,134$ & $7,566,727$ \\
\hline $\mathbf{N}$ & 82 & 85 & 97 & 101 & 104 & 102 & 105 & 105 & 106 & 110 \\
\hline \multicolumn{11}{|c|}{ Electronic Resources Expenditures as a Percent of Total Materials Expenditures } \\
\hline Average & 3.60 & 4.75 & 6.39 & 6.83 & 7.76 & 8.85 & 10.56 & 12.88 & 16.25 & 19.60 \\
\hline Median & N/A & 4.45 & 5.33 & 6.42 & 7.51 & 8.29 & 10.18 & 12.75 & 14.80 & 18.15 \\
\hline $\mathbf{N}$ & 82 & 85 & 97 & 101 & 104 & 102 & 105 & 105 & 106 & 110 \\
\hline \multicolumn{11}{|c|}{ Expenditures for Bibliographic Utilities, Networks, etc. (External) } \\
\hline Total & N/A & N/A & N/A & N/A & $\$ 3,827,348$ & $4,695,737$ & $7,442,962$ & $9,523,348$ & $14,655,078$ & $20,373,560$ \\
\hline Average & N/A & N/A & N/A & N/A & $\$ 136,691$ & 142,295 & 201,161 & 250,614 & 311,810 & 424,449 \\
\hline Median & N/A & $\mathrm{N} / \mathrm{A}$ & N/A & $\mathrm{N} / \mathrm{A}$ & $\$ 120,096$ & 128,795 & 145,280 & 204,598 & 198,289 & 336,690 \\
\hline $\mathbf{N}$ & & & & & 28 & 33 & 37 & 38 & 47 & 48 \\
\hline
\end{tabular}

*Figures reflect Materials Expenditures only from those institutions that reported nonzero figures for Total Electronic Resources. 


\section{TABLE 7B}

\section{Electronic Resources and Materials Expenditures in ARL University Libraries, 2002-2009}

\begin{tabular}{|c|c|c|c|c|c|c|c|}
\hline & 2002-2003 & 2003-2004 & 2004-2005 & 2005-2006 & 2006-2007 & 2007-2008 & 2008-2009 \\
\hline \multicolumn{8}{|c|}{ a. Computer File Expenditures (monographic/onetime) } \\
\hline Total & $\$ 23,275,683$ & $\$ 32,098,404$ & $\$ 38,744,076$ & $\$ 48,793,981$ & $\$ 59,808,658$ & $\$ 73,102,024$ & $\$ 69,148,203$ \\
\hline Average & $\$ 225,978$ & $\$ 314,690$ & $\$ 372,539$ & $\$ 478,372$ & $\$ 558,959$ & $\$ 676,574$ & $\$ 628,620$ \\
\hline Median & $\$ 111,266$ & $\$ 191,148$ & $\$ 210,576$ & $\$ 336,338$ & $\$ 352,802$ & $\$ 410,202$ & $\$ 363,746$ \\
\hline $\mathbf{N}$ & 103 & 102 & 104 & 102 & 107 & 108 & 110 \\
\hline \multicolumn{8}{|c|}{ b. Electronic Serial Expenditures } \\
\hline Total & $\$ 205,300,292$ & $\$ 269,601,241$ & $\$ 328,166,027$ & $\$ 383,127,163$ & $\$ 476,225,086$ & $\$ 554,637,844$ & $\$ 637,458,376$ \\
\hline Average & $\$ 1,849,552$ & $\$ 2,450,920$ & $\$ 3,038,574$ & $\$ 3,547,474$ & $\$ 4,290,316$ & $\$ 5,042,162$ & $\$ 5,691,593$ \\
\hline Median & $\$ 1,649,361$ & $\$ 2,348,463$ & $\$ 2,824,962$ & $\$ 3,349,709$ & $\$ 4,240,530$ & $\$ 4,899,366$ & $\$ 5,337,237$ \\
\hline $\mathbf{N}$ & 111 & 110 & 108 & 108 & 111 & 110 & 112 \\
\hline \multicolumn{8}{|c|}{ c. Total Electronic Resources (Total $a+b)$} \\
\hline Total & $\$ 228,575,974$ & $\$ 301,699,645$ & $\$ 366,910,103$ & $\$ 431,921,144$ & $\$ 536,033,744$ & $\$ 627,707,869$ & $\$ 706,606,579$ \\
\hline Average & $\$ 2,059,243$ & $\$ 2,718,015$ & $\$ 3,366,147$ & $\$ 3,962,579$ & $\$ 4,786,016$ & $\$ 5,655,026$ & $\$ 6,253,156$ \\
\hline Median & $\$ 1,775,865$ & $\$ 2,705,847$ & $\$ 3,144,841$ & $\$ 3,792,873$ & $\$ 4,661,123$ & $\$ 5,410,421$ & $\$ 5,854,147$ \\
\hline $\mathbf{N}$ & 111 & 111 & 109 & 109 & 112 & 111 & 113 \\
\hline \multicolumn{8}{|c|}{ Total Library Materials Expenditures* } \\
\hline Total & $\$ 950,275,167$ & $\$ 1,016,121,605$ & $\$ 1,031,619,722$ & $\$ 1,109,340,878$ & $\$ 1,213,082,817$ & $\$ 1,279,690,962$ & $\$ 1,315,122,261$ \\
\hline Average & $\$ 8,561,038$ & $\$ 9,154,249$ & $\$ 9,464,401$ & $\$ 10,177,439$ & $\$ 10,831,097$ & $\$ 11,528,747$ & $\$ 11,638,250$ \\
\hline Median & $\$ 7,707,153$ & $\$ 8,276,175$ & $\$ 8,662,668$ & $\$ 9,156,974$ & $\$ 9,597,677$ & $\$ 10,416,077$ & $\$ 10,364,778$ \\
\hline $\mathbf{N}$ & 111 & 111 & 109 & 109 & 112 & 111 & 113 \\
\hline \multicolumn{8}{|c|}{ Electronic Resources Expenditures as a Percent of Total Materials Expenditures } \\
\hline Average & 25.02 & 31.33 & 37.46 & 40.93 & 46.55 & 51.46 & 56.33 \\
\hline Median & 22.01 & 29.81 & 37.53 & 43.14 & 47.68 & 53.06 & 57.03 \\
\hline $\mathbf{N}$ & 111 & 111 & 109 & 109 & 112 & 111 & 113 \\
\hline \multicolumn{8}{|c|}{ Expenditures for Bibliographic Utilities, Networks, etc. (External) } \\
\hline Total & $\$ 21,470,716$ & $\$ 17,420,520$ & $\$ 16,082,790$ & $\$ 15,930,476$ & $\$ 18,931,797$ & $\$ 21,079,241$ & $\$ 21,695,047$ \\
\hline Average & $\$ 438,178$ & $\$ 335,010$ & $\$ 349,626$ & $\$ 318,610$ & $\$ 225,379$ & $\$ 242,290$ & $\$ 235,816$ \\
\hline Median & $\$ 250,000$ & $\$ 94,837$ & $\$ 149,396$ & $\$ 143,649$ & $\$ 33,247$ & $\$ 54,750$ & $\$ 44,745$ \\
\hline $\mathbf{N}$ & 49 & 52 & 46 & 50 & 84 & 87 & 92 \\
\hline
\end{tabular}

* Figures reflect Materials Expenditures only from those institutions that reported nonzero figures for Total Electronic Resources. The 2006-2007 and 2007-2008 figures have been revised. 


\section{ARL Statistics ${ }^{\circledR}$ Interactive Analytics}

ARL Statistics ${ }^{\circledR}$ Interactive Analytics is a subscription-based service for non ARL member libraries, for-profit and not-forprofit entities, and researchers who wish to access the ARL Statistics ${ }^{\circledR}$ Analytics.

Librarians at ARL member libraries: Please get in touch with your ARL Statistics Primary Contact for access to the ARL Statistics ${ }^{\circledR}$. This person can facilitate access to the ARL Statistics ${ }^{\circledR}$ system for you.

ARL Statistics ${ }^{\circledR}$ Interactive Analytics is a service that interactively analyzes ARL Statistics ${ }^{\circledR}$ data. ARL Statistics ${ }^{\circledR}$ Interactive $^{-1}$ Analytics will allow you to:

- review the library data collected by ARL

- generate rankings of institutions by selected criteria

- create graphs from the data

- generate summary statistics for all ARL libraries

- download the data by year in spreadsheet format

- review the ARL indices

ARL is offering this subscription service to non-member organizations and individuals that are interested in accessing the final verified data through an interactive interface. (All ARL member libraries have access to the ARL Statistics ${ }^{\circledast}$ Analytics as soon as the data are submitted through the StatsQUAL ${ }^{\circledR}$ password-protected gateway at arlstatistics.org).

The subscription fee is $\$ 500$ for non-profit organizations and $\$ 750$ for others. Interested organizations may email stats@ arl.org.

ARL Statistics ${ }^{\circledR}$ is a series of annual publications that describe the collections, expenditures, staffing, and service activities for the member libraries of the Association of Research Libraries. Statistics have been collected and published annually for the members of the Association since 1961-62. Before that, annual statistics for university libraries were collected by James Gerould, first at Minnesota and later at Princeton. These data cover the years 1907-08 through 196162 and are now called the Gerould statistics. The whole data series from 1908 to the present represents the oldest and most comprehensive continuing library statistical series in North America. Print copies may be ordered from the ARL Publications Program.

For more information, regarding the machine-readable datafiles, analysis of research library trends, and full-text of the ARL Statistics publications among other things, see: http://www.arlstatistics.org.

We would like to thank the University of Virginia for supporting the ARL Statistics Interactive edition from 1995 to 2010. Special thanks to Texas A\&M University and the University of Texas for enabling ARL to support data curation activities like the ARL Statistics ${ }^{\circledR}$ Interactive Analytics through their support and development of LibQUAL $+{ }^{\circledR}$ Analytics. 


\section{ARL Library Data Tables}

\section{8-2009}




\section{Collections: Volumes and Monographs}

Volumes In Volumes Added Volumes Added Library (Gross) (Net)
Titles Held

(1b)
(2)
Monographs Purchased

(Volumes)

(Survey Question Number)

(1)

(1b.i)

(1b)

INSTITUTION

Notes

\begin{tabular}{|c|c|c|c|c|c|c|}
\hline ALABAMA & bGLM+ & $3,396,810$ & 92,417 & 82,812 & $2,571,076$ & 98,779 \\
\hline ALBERTA & GLM+ & $7,066,429$ & 191,949 & 175,750 & $3,314,868$ & $\mathrm{UA} / \mathrm{NA}$ \\
\hline ARIZONA & bGLM+ & $5,794,299$ & 149,544 & 72,019 & $5,560,453$ & 35,650 \\
\hline ARIZONA STATE & BGL+ & $4,393,156$ & 52,991 & 38,992 & UA/NA & 32,851 \\
\hline AUBURN & + & $3,459,542$ & 26,083 & 24,677 & $3,136,841$ & 11,277 \\
\hline BOSTON & $\mathrm{LM}+$ & $2,864,562$ & 39,771 & 17,488 & $2,482,067$ & 19,425 \\
\hline BOSTON COLLEGE & $\mathrm{L}+$ & $2,555,641$ & 46,551 & 38,973 & $2,045,526$ & 28,152 \\
\hline BRIGHAM YOUNG & GL+ & $4,168,102$ & 100,227 & 88,023 & $3,458,192$ & 72,501 \\
\hline BRITISH COLUMBIA & $\mathrm{LM}+$ & $6,312,477$ & 142,939 & 94,216 & $3,726,961$ & 38,955 \\
\hline BROWN & bGM+ & $3,936,274$ & 79,769 & 74,207 & $2,622,422$ & 44,711 \\
\hline CALGARY & GLM+ & $3,244,903$ & 130,361 & 109,524 & $2,088,424$ & 44,137 \\
\hline CALIFORNIA, BERKELEY & $\mathrm{L}+$ & $11,026,554$ & 162,733 & 141,752 & UA/NA & $\mathrm{UA} / \mathrm{NA}$ \\
\hline CALIFORNIA, DAVIS & GLM+ & $4,156,170$ & 86,268 & 81,315 & $2,136,607$ & 65,603 \\
\hline CALIFORNIA, IRVINE & bGM+ & $3,145,926$ & 99,232 & 96,220 & $2,107,463$ & 35,425 \\
\hline CALIFORNIA, LOS ANGELES & bGLM+ & $9,045,818$ & 127,477 & 119,440 & UA/NA & 50,170 \\
\hline CALIFORNIA, RIVERSIDE & G+ & $2,955,171$ & 47,329 & 46,588 & $2,291,248$ & 11,849 \\
\hline CALIFORNIA, SAN DIEGO & bGM+ & $3,651,393$ & 138,786 & 37,900 & UA/NA & 36,171 \\
\hline CALIFORNIA, SANTA BARBARA & G+ & $2,996,397$ & 55,527 & 47,398 & $2,850,203$ & 19,509 \\
\hline CASE WESTERN RESERVE & GLM+ & $2,777,529$ & 38,737 & 25,867 & $2,135,777$ & 8,025 \\
\hline CHICAGO & bGLM+ & $8,830,151$ & 261,776 & 232,992 & $5,793,778$ & 129,636 \\
\hline CINCINNATI & GLM+ & $3,715,957$ & 85,635 & 20,309 & $2,341,343$ & 34,035 \\
\hline COLORADO & bG+ & $4,348,639$ & 82,423 & 74,315 & UA/NA & 64,165 \\
\hline COLORADO STATE & G+ & $2,366,608$ & 83,411 & 78,376 & $2,307,611$ & 70,125 \\
\hline COLUMBIA & GLM+ & $10,449,223$ & 243,094 & 152,407 & $7,059,240$ & 167,270 \\
\hline CONNECTICUT & BbGLM+ & $3,982,991$ & 53,415 & 4,372 & $2,304,339$ & 15,673 \\
\hline CORNELL & BGL+ & $8,036,029$ & 130,331 & 130,331 & $4,963,660$ & $\mathrm{UA} / \mathrm{NA}$ \\
\hline DARTMOUTH & bGM+ & $2,848,521$ & 68,462 & 62,596 & $2,182,194$ & 54,650 \\
\hline DELAWARE & G+ & $2,807,445$ & 37,300 & 28,174 & $2,113,639$ & 22,798 \\
\hline DUKE & LM+ & $6,031,761$ & 145,399 & 86,535 & . & 78,817 \\
\hline EMORY & BLM+ & $3,479,536$ & 91,504 & 79,981 & $2,112,955$ & 49,794 \\
\hline FLORIDA & BbLM+ & $4,299,252$ & 51,159 & 11,134 & UA/NA & 29,994 \\
\hline FLORIDA STATE & BbLM+ & $3,034,491$ & 43,204 & 3,658 & $2,505,345$ & 17,143 \\
\hline GEORGE WASHINGTON & BGLM+ & $2,268,571$ & 76,171 & 29,644 & $1,460,655$ & 29,593 \\
\hline GEORGETOWN & bGLM+ & $3,431,948$ & 76,926 & 60,070 & $3,001,874$ & 38,265 \\
\hline GEORGIA & GL+ & $4,716,401$ & 84,117 & 79,110 & $3,551,110$ & 41,517 \\
\hline GEORGIA TECH & G+ & $2,541,880$ & 21,489 & 17,209 & 798,988 & 9,264 \\
\hline GUELPH & BG+ & $1,898,348$ & 47,313 & 44,720 & $1,507,647$ & 12,992 \\
\hline HARVARD & GLM+ & $16,557,002$ & 349,050 & 302,247 & UA/NA & $\mathrm{UA} / \mathrm{NA}$ \\
\hline HAWAII & GLM+ & $3,588,005$ & 68,517 & 54,334 & $2,270,600$ & 25,801 \\
\hline
\end{tabular}




\section{Collections: Volumes and Monographs}

Volumes In Volumes Added Volumes Added Library

(Net)
(Gross)

(1)

(1b.i)

Titles Held

Monographs Purchased

(Volumes)

(Survey Question Number)

Notes

INSTITUTION

HOUSTON

bL+

HOWARD

bLM+

$2,666,072$

(1b)

(2)

(3)

ILLINOIS, CHICAGO

ILLINOIS, URBANA

M+

$2,589,888$

53,041

2,515,007

22,043

48,790

2,560,705

20,009

$\mathrm{M}+$

$12,780,067$

INDIANA

bGL+

$8,543,025$

$5,155,258$

$\mathrm{LM}+$

$2,578,144$

IOWA STATE

G+

JOHNS HOPKINS

\section{BbGM+}

$3,737,404$

KANSAS

BbGLM+

$4,271,113$

KENT STATE

$\mathrm{BbG}+$

KENTUCKY

GLM+

$2,889,013$

LAVAL

GLM+

$3,784,382$

$2,966,168$

LOUISIANA STATE

\section{LOUISVILLE}

MCGILL

MCMASTER

GLM+

4,112,774

\section{BGM+}

2,234,328

BbGLM+

$4,128,321$

bGM+

2,014,067

GLM+

$2,317,417$

MANITOBA

G+

MASSACHUSETTS

G+

$3,767,653$

MIT

MIAMI

$$
\text { G+ }
$$

$3,654,181$

bGLM+

$3,057,604$

\section{MICHIGAN}

MICHIGAN STATE

GLM+

$3,300,370$

GL+

9,575,256

MINNESOTA

bGLM+

$5,292,806$

MISSOURI

MONTREAL

GLM+

$6,975,576$

\section{GLM+}

3,523,795

NEBRASKA

NEW MEXICO

L+

NEW YORK

NORTH CAROLINA

NORTH CAROLINA STATE

NORTHWESTERN

NOTRE DAME

$\mathrm{OHIO}$

OHIO STATE

OKLAHOMA

OKLAHOMA STATE

OREGON

PENNSYLVANIA

\section{GLM+}

$3,180,763$

$3,247,311$

\section{bGLM+}

$3,117,590$

GLM+

$5,191,617$

$6,735,325$

G+

$4,158,190$

BGLM+

4,930,613

bGL+

$3,469,001$

BbGM+

$2,958,684$

bGLM+

$6,206,443$

GLM+

$5,433,036$

BGM+

$2,932,910$

BGL+

$3,006,119$

GLM+

$6,223,214$

18,961

UA/NA

4,488

265,766

261, 197

146,141

174,623

135,794

$7,138,003$

40,237

132,645

84,088

52,154

141,069

48,757

40,720

$4,603,721$

28,369

$1,609,977$

28,280

37,288

6,965

44,662

35,571

42,834

66,453

35,057

$3,815,955$

37,475

14,948

89,696

64,834

71,459

38,028

27,137

67,550

43,237

60,672

121,576

178,991

122,074

80,699

33,133

50,793

75,428

80,866

53,731

40,570

72,196

176,363

36,074

102,868

81,306

144,001

73,282

59,398

44,309

29,325

58,854

50,223

119,995

138,256

137,419

78,480

89,452

84,037

85,644

139,870

143,603

66,157

49,286

135,456

4,908

43,253

104,010

112,102

119,161

76,912

83,784

75,711

5,314

$-79,003$

133,112

65,044

46,380

126,626

$1,735,210$

33,763

40,212

$1,366,649$

30,989

35,901

UA/NA

201,983

53,891

123,082

117,610

UA/NA

11,286

UA/NA

22,669

40,438

2,331,942

144,173

$9,076,153$

23,397

37,445

$4,757,000$

33,643

2,760,594

61,439

$1,956,602$

9,923

25,253

51,828

67,691

21,544

2,036,334

47,683

45,172

28,737

73,102

43,015

$3,271,077$

57,964

16,761

$1,885,765$

UA/NA 


\section{Collections: Volumes and Monographs}

Volumes In Volumes Added Volumes Added Library (Gross) (Net)
Titles Held

(1b)
(2)
Monographs Purchased

(Volumes)

(Survey Question Number)

(1)

(1b.i)

(1b)

INSTITUTION

Notes

\begin{tabular}{|c|c|c|c|c|c|c|}
\hline PENNSYLVANIA STATE & BbGLM+ & $5,365,489$ & 120,191 & 10,844 & $4,206,341$ & 60,919 \\
\hline PITTSBURGH & BGLM+ & $5,897,931$ & 209,556 & 184,168 & UA/NA & $\mathrm{UA} / \mathrm{NA}$ \\
\hline PRINCETON & bG+ & $7,075,441$ & 147,989 & 134,187 & $4,983,404$ & 123,349 \\
\hline PURDUE & G+ & $2,506,059$ & 33,134 & $-3,097$ & $2,012,865$ & 13,774 \\
\hline QUEEN'S & $\mathrm{LM}+$ & $2,577,143$ & 42,345 & 34,879 & $\mathrm{UA} / \mathrm{NA}$ & 23,587 \\
\hline RICE & G+ & $2,620,342$ & 50,233 & 49,574 & $2,109,632$ & 40,286 \\
\hline ROCHESTER & $\mathrm{bM}+$ & $3,740,714$ & 31,204 & 25,329 & $2,825,844$ & 21,022 \\
\hline RUTGERS & BGL+ & $4,570,477$ & 77,196 & 40,561 & $2,003,233$ & 21,378 \\
\hline SASKATCHEWAN & GLM+ & $2,361,589$ & 44,830 & 4 & $1,714,978$ & 17,050 \\
\hline SOUTH CAROLINA & $\mathrm{LM}+$ & $3,675,054$ & 52,810 & 47,364 & $3,361,206$ & 14,256 \\
\hline SOUTHERN CALIFORNIA & BGLM+ & $4,124,253$ & 55,717 & 39,969 & $2,579,159$ & 53,645 \\
\hline SOUTHERN ILLINOIS & $\mathrm{LM}+$ & $3,149,701$ & 28,048 & 15,341 & $1,987,072$ & 13,338 \\
\hline SUNY-ALBANY & G+ & $2,263,767$ & 48,907 & 38,526 & $1,463,144$ & 16,065 \\
\hline SUNY-BUFFALO & GLM+ & $3,852,074$ & 176,048 & 170,972 & $2,421,423$ & 23,160 \\
\hline SUNY-STONY BROOK & $\mathrm{bM}+$ & $2,277,714$ & 18,342 & 12,148 & $1,284,318$ & 4,780 \\
\hline SYRACUSE & GL+ & $3,201,031$ & 60,184 & 26,331 & $1,845,727$ & 21,678 \\
\hline TEMPLE & BGLM+ & $3,761,933$ & 63,741 & 61,288 & $2,065,440$ & 38,720 \\
\hline TENNESSEE & BbGLM+ & $3,322,418$ & 61,859 & 43,776 & $2,175,328$ & 45,399 \\
\hline TEXAS & GL+ & $9,853,414$ & 133,562 & 93,368 & $5,335,217$ & 61,470 \\
\hline TEXAS A\&M & $\mathrm{BbG}+$ & $4,088,969$ & 171,127 & 155,434 & $3,079,479$ & 144,623 \\
\hline TEXAS TECH & BLM+ & $2,675,872$ & 53,130 & 50,068 & UA/NA & 35,323 \\
\hline TORONTO & BLM+ & $11,345,102$ & 239,548 & 158,614 & $6,569,831$ & 158,826 \\
\hline TULANE & BGLM+ & $4,004,458$ & 114,847 & 112,615 & $\mathrm{UA} / \mathrm{NA}$ & 638,592 \\
\hline UTAH & $\mathrm{LM}+$ & $3,418,976$ & 55,420 & 51,165 & UA/NA & $\mathrm{UA} / \mathrm{NA}$ \\
\hline VANDERBILT & GLM+ & $3,467,542$ & 52,496 & 27,189 & $2,653,228$ & 22,963 \\
\hline VIRGINIA & LM+ & $5,605,891$ & 141,305 & 102,343 & $4,016,275$ & 65,765 \\
\hline VIRGINIA TECH & G+ & $2,385,815$ & 60,364 & 54,760 & $1,555,134$ & 18,119 \\
\hline WASHINGTON & BGLM+ & $7,549,765$ & 172,772 & 140,544 & $4,599,418$ & 62,391 \\
\hline WASHINGTON STATE & $\mathrm{BbG}+$ & $2,394,849$ & 54,112 & 52,095 & $2,017,135$ & 13,767 \\
\hline WASHINGTON U.-ST. LOUIS & GLM+ & $4,281,213$ & 64,505 & 56,326 & $2,984,769$ & 30,637 \\
\hline WATERLOO & G+ & $2,310,601$ & 35,057 & 16,581 & $1,807,906$ & 32,253 \\
\hline WAYNE STATE & GLM+ & $3,665,628$ & 23,678 & 4,986 & $2,251,350$ & 16,889 \\
\hline WESTERN ONTARIO & GL+ & $3,644,679$ & 99,162 & 98,183 & $2,410,951$ & 50,990 \\
\hline WISCONSIN & GLM+ & $8,310,732$ & 134,118 & 100,803 & $5,668,964$ & 77,018 \\
\hline YALE & GLM+ & $12,564,157$ & 292,324 & 280,563 & $8,024,854$ & 206,555 \\
\hline YORK & BGL+ & $2,910,610$ & 84,072 & 76,861 & $1,936,767$ & 25,659 \\
\hline BOSTON PUBLIC & bG+ & $7,738,398$ & 106,408 & 106,408 & $\mathrm{UA} / \mathrm{NA}$ & 95,767 \\
\hline CENTER FOR RESEARCH LIBRARIES & & $2,241,444$ & 12,868 & 12,868 & $\mathrm{UA} / \mathrm{NA}$ & 4,395 \\
\hline LIBRARY OF CONGRESS & bG+ & $21,586,006$ & 367,598 & 367,598 & $12,388,074$ & 152,328 \\
\hline
\end{tabular}


Collections: Volumes and Monographs

Volumes In Volumes Added Volumes Added Library

(Survey Question Number)

(1b.i)
(1)
(Net)

(1b)
Titles Held

896

73,138

36,886

139,182

26,303

$2,737,317$

56,146

G+

$1,406,220$

G - Government documents not included in serials count

B - Includes branch campuses

$\mathrm{b}$ - Basis of volume count is bibliographic
U/A - Unavailable

N/A - Not Applicable

. - no data supplied

\section{Summary Data}

(Survey Question Number)

University Libraries

Mean

Median

High

Low

Totals

Number of Libraries Reporting

\section{Nonuniversity Libraries}

Median

$2,627,858$

$50,125,543$

Number of Libraries Reporting
Volumes In Volumes Added Volumes Added Library (Gross) (Net)

(1)

(1b.i)

(1b)

(2)

(3)

$\begin{array}{rr}4,528,262 & 95,157 \\ 3,659,905 & 77,838 \\ 16,557,002 & 349,050 \\ 1,898,348 & 18,342 \\ 516,221,820 & 10,847,894 \\ 114 & 114\end{array}$

7,838

71,240

57,824

302,247

$-79,003$

$8,121,381$

114
33,582
745,690
9

$8,867,071$
Titles Held

Monographs Purchased (Volumes)

53,292

35,901

638,592

4,488

$5,702,255$

107

$278,802,849$

93

9,201

376,783

$24,528,722$

6

9

$566,347,363$

$11,605,682$

$303,331,571$

$6,079,038$ 


\section{Collections: Current Serials}

$\begin{array}{rrr}\text { Current Serials } & \text { Current Serials } & \text { Current Serials } \\ \text { Total } & \text { Purchased } & \text { Purchased }\end{array}$

Electronic

Current Serials Purchased Print

(Survey Question Number)

(5)

(5a)

(5a.i)

(5a.ii)

INSTITUTION

ALABAMA

ALBERTA

ARIZONA

ARIZONA STATE

AUBURN

BOSTON

BOSTON COLLEGE

BRIGHAM YOUNG

BRITISH COLUMBIA

BROWN

CALGARY

CALIFORNIA, BERKELEY

CALIFORNIA, DAVIS

CALIFORNIA, IRVINE

CALIFORNIA, LOS ANGELES

CALIFORNIA, RIVERSIDE

CALIFORNIA, SAN DIEGO

CALIFORNIA, SANTA BARBARA

CASE WESTERN RESERVE

CHICAGO

CINCINNATI

COLORADO

COLORADO STATE

COLUMBIA

CONNECTICUT

CORNELL

DARTMOUTH

DELAWARE

DUKE

EMORY

FLORIDA

FLORIDA STATE

GEORGE WASHINGTON

GEORGETOWN

GEORGIA

GEORGIA TECH

GUELPH

HARVARD

HAWAII

Notes

\begin{tabular}{|c|c|c|c|c|}
\hline bGLM+ & 81,462 & 51,665 & 46,676 & 4,989 \\
\hline GLM+ & 114,966 & 101,539 & 93,424 & 8,115 \\
\hline bGLM+ & 84,367 & 79,985 & 72,927 & 7,058 \\
\hline BGL+ & 95,515 & 78,420 & 72,624 & 5,796 \\
\hline+ & 50,011 & 19,437 & 18,646 & 791 \\
\hline LM+ & 57,755 & 49,030 & 39,587 & 9,443 \\
\hline L+ & 37,279 & 23,129 & 18,838 & 4,291 \\
\hline GL+ & 77,646 & 48,793 & 39,243 & 9,550 \\
\hline LM+ & 82,202 & 82,202 & 67,255 & 14,947 \\
\hline bGM+ & 65,908 & 45,669 & 43,077 & 2,592 \\
\hline GLM+ & 48,648 & 36,040 & 28,554 & 7,486 \\
\hline L+ & 106,108 & 72,944 & 36,538 & 36,406 \\
\hline GLM+ & 73,912 & 53,466 & 43,235 & 10,231 \\
\hline bGM+ & 53,891 & 35,957 & 32,352 & 3,605 \\
\hline bGLM+ & 38,975 & 23,504 & 8,217 & 15,287 \\
\hline G+ & 90,153 & 62,029 & 59,269 & 2,760 \\
\hline bGM+ & 84,513 & 61,211 & 34,429 & 26,782 \\
\hline G+ & 69,327 & 35,259 & 29,483 & 5,776 \\
\hline GLM+ & 75,083 & 65,734 & 60,646 & 5,088 \\
\hline bGLM+ & 102,767 & 59,714 & 46,586 & 13,128 \\
\hline GLM+ & 103,066 & 86,563 & 80,814 & 5,749 \\
\hline bG+ & 60,805 & 39,978 & 36,352 & 3,626 \\
\hline G+ & 52,433 & 29,664 & 27,883 & 1,781 \\
\hline GLM+ & 144,787 & 115,050 & 98,469 & 16,581 \\
\hline BbGLM+ & 92,884 & 50,436 & 46,119 & 4,317 \\
\hline BGL+ & 102,000 & UA/NA & UA/NA & UA/NA \\
\hline bGM+ & 64,745 & 58,203 & 53,301 & 4,902 \\
\hline G+ & 31,706 & 21,685 & 19,768 & 1,917 \\
\hline LM+ & 91,931 & 57,365 & 45,785 & 11,580 \\
\hline BLM+ & 83,514 & 64,321 & 56,241 & 8,080 \\
\hline BbLM+ & 89,741 & 49,186 & 27,087 & 22,099 \\
\hline BbLM+ & 78,295 & 56,471 & 52,201 & 4,270 \\
\hline BGLM+ & 74,329 & 64,311 & 58,518 & 5,793 \\
\hline bGLM+ & 70,518 & 62,196 & 56,038 & 6,158 \\
\hline GL+ & 96,401 & 66,472 & 59,168 & 7,304 \\
\hline G+ & 41,412 & 10,754 & 8,020 & 2,734 \\
\hline BG+ & 51,566 & 46,691 & 44,766 & 1,925 \\
\hline GLM+ & 132,107 & UA/NA & UA/NA & $\mathrm{UA} / \mathrm{NA}$ \\
\hline GLM+ & 67,863 & 42,276 & 31,588 & 10,688 \\
\hline
\end{tabular}




\section{Collections: Current Serials}

Current Serials Current Serials

Not Purchased

(5b)
Not Purchased Consortial

(5b.i)
Current Serials

Not Purchased

Freely Accessible
Current Serials

Not Purchased

Print

(5b.iii)
Current Serials

Not Purchased GovDocs

(5b.iv) (Survey Question Number)

INSTITUTION

\begin{tabular}{|c|c|c|c|c|c|}
\hline 29,797 & 14 & 28,034 & 524 & 1,225 & ALABAMA \\
\hline 13,427 & UA/NA & $\mathrm{UA} / \mathrm{NA}$ & 13,427 & 0 & ALBERTA \\
\hline 4,382 & UA/NA & 3,844 & 538 & 0 & ARIZONA \\
\hline 17,095 & UA/NA & 4,018 & 468 & 12,609 & ARIZONA STATE \\
\hline 30,574 & 14,317 & 8,737 & 839 & 6,681 & AUBURN \\
\hline 8,725 & 4,361 & 4,168 & 39 & 157 & BOSTON \\
\hline 14,150 & 8,729 & 5,238 & 183 & UA/NA & BOSTON COLLEGE \\
\hline 28,853 & UA/NA & 27,600 & 1,079 & 174 & BRIGHAM YOUNG \\
\hline UA/NA & UA/NA & UA/NA & UA/NA & UA/NA & BRITISH COLUMBIA \\
\hline 20,239 & 0 & 7,605 & 867 & 11,767 & BROWN \\
\hline 12,608 & 6,815 & 4,117 & 173 & 1,503 & CALGARY \\
\hline 33,164 & UA/NA & 26,807 & 6,357 & UA/NA & CALIFORNIA, BERKELEY \\
\hline 20,446 & 0 & 13,658 & 5,778 & 1,010 & CALIFORNIA, DAVIS \\
\hline 17,934 & UA/NA & 3,811 & 86 & 14,037 & CALIFORNIA, IRVINE \\
\hline 15,471 & 0 & 6,295 & 6,784 & 2,392 & CALIFORNIA, LOS ANGELES \\
\hline 28,124 & 18,049 & 683 & 358 & 9,034 & CALIFORNIA, RIVERSIDE \\
\hline 23,302 & UA/NA & $\mathrm{UA} / \mathrm{NA}$ & 12,708 & 10,594 & CALIFORNIA, SAN DIEGO \\
\hline 34,068 & UA/NA & 26,782 & 1,489 & 5,797 & CALIFORNIA, SANTA BARBARA \\
\hline 9,349 & 72 & 4,994 & 86 & 4,197 & CASE WESTERN RESERVE \\
\hline 43,053 & 1,822 & 17,143 & 12,089 & 11,999 & CHICAGO \\
\hline 16,503 & 0 & 8,787 & 0 & 7,716 & CINCINNATI \\
\hline 20,827 & 0 & 5,947 & 171 & 14,709 & COLORADO \\
\hline 22,769 & 3,553 & 18,621 & 286 & 309 & COLORADO STATE \\
\hline 29,737 & 0 & 15,748 & 6,355 & 7,634 & COLUMBIA \\
\hline 42,448 & 21,283 & 8,371 & 273 & 12,521 & CONNECTICUT \\
\hline UA/NA & UA/NA & UA/NA & UA/NA & UA/NA & CORNELL \\
\hline 6,542 & UA/NA & 4,076 & 1,375 & 1,091 & DARTMOUTH \\
\hline 10,021 & 0 & 2,266 & 262 & 7,493 & DELAWARE \\
\hline 34,566 & 3,281 & 29,482 & 396 & 1,407 & DUKE \\
\hline 19,193 & 6 & 16,870 & 106 & 2,211 & EMORY \\
\hline 40,555 & 17,566 & 19,832 & 3,066 & 91 & FLORIDA \\
\hline 21,824 & 13,363 & 6,394 & 911 & 1,156 & FLORIDA STATE \\
\hline 10,018 & 7,590 & 1,441 & 4 & 983 & GEORGE WASHINGTON \\
\hline 8,322 & UA/NA & 6,929 & 1,393 & $\mathrm{UA} / \mathrm{NA}$ & GEORGETOWN \\
\hline 29,929 & 16,641 & 5,528 & 739 & 7,021 & GEORGIA \\
\hline 30,658 & 0 & 5,758 & 1,136 & 23,764 & GEORGIA TECH \\
\hline 4,875 & UA/NA & 4,875 & $\mathrm{UA} / \mathrm{NA}$ & UA/NA & GUELPH \\
\hline UA/NA & UA/NA & UA/NA & UA/NA & UA/NA & HARVARD \\
\hline 25,587 & 0 & 13,670 & 6,797 & 5,120 & HAWAII \\
\hline
\end{tabular}




\section{Collections: Current Serials}

$\begin{array}{rrr}\text { Current Serials } & \text { Current Serials } & \text { Current Serials } \\ \text { Total } & \text { Purchased } & \text { Purchased }\end{array}$

Electronic

Current Serials Purchased Print

(Survey Question Number)

(5)

(5a)

(5a.i)

(5a.ii)

\section{INSTITUTION}

HOUSTON

HOWARD

ILLINOIS, CHICAGO

ILLINOIS, URBANA

INDIANA

IOWA

IOWA STATE

JOHNS HOPKINS

KANSAS

KENT STATE

KENTUCKY

LAVAL

LOUISIANA STATE

LOUISVILLE

MCGILL

MCMASTER

MANITOBA

MARYLAND

MASSACHUSETTS

MIT

MIAMI

MICHIGAN

MICHIGAN STATE

MINNESOTA

MISSOURI

MONTREAL

NEBRASKA

NEW MEXICO

NEW YORK

NORTH CAROLINA

NORTH CAROLINA STATE

NORTHWESTERN

NOTRE DAME

$\mathrm{OHIO}$

OHIO STATE

OKLAHOMA

OKLAHOMA STATE

OREGON

PENNSYLVANIA
Notes

bL+

bLM+

$\mathrm{M}+$

bGL+

L+

LM+

G+

BbGM+

BbGLM+

$\mathrm{BbG}+$

GLM+

GLM+

GLM+

BGM+

BbGLM+

bGM+

GLM+

G+

G+

G+

G+

bGLM+

GLM+

GL+

bGLM+

GLM+

GLM+

L+

\section{GLM+}

bGLM+

GLM+

G+

BGLM+

bGL+

BbGM+

bGLM+

GLM+

BGM+

BGL+

GLM+

81,264

24,307

67,318

117,517

84,933

72,271

97,352

100,074

73,613

79,237

78,194

49,181

15,204

40,100

108,089

72,176

55,018

64,626

61,598

57,674

57,817

43,730

46,196

104,177

86,627

65,536

69,781

52,115

51,989

53,170

60,105

76,869

70,047

106,026

90,860

46,543

90,160

40,288

\section{7,094}

108,454

80,132

63,271

98,844

88,352

46,823

90,156

69,621

70,803

46,879

72,688
46,196
97,524

86,077

49,877

39,726

26,021

23,039

37,277

41,996

71,223

UA/NA

74,459

70,597

24,857

82,785

33,000

58,917

75,188

51,091

32,369

68,751

66,017

29,544

79,751

57,803

55,672

23,421

62,731

44,851

7,278

36,601

58,140

51,119

38,550

54,225

56,586

52,512

54,637

40,151

39,826

96,510

73,959

44,598

37,015

23,534

16,961

34,877

38,040

67,604

UA/NA

62,997

47,632

18,917

75,282

24,765

56,449

64,915

44,682

31,022

UA/NA

UA/NA

27,004

71,142

53,405

52,572

19,700

47,698 


\section{Collections: Current Serials}

Current Serials Current Serials Not Purchased

(5b)
Not Purchased Consortial
Current Serials

Not Purchased

Freely Accessible

(5b.ii)

Current Serials

Not Purchased

Print

(5b.iii)
Current Serials

Not Purchased GovDocs

(5b.iv) (Survey Question Number)

INSTITUTION

\begin{tabular}{|c|c|c|c|c|c|}
\hline 32,083 & 14,449 & 4,711 & 3,671 & 9,252 & HOUSTON \\
\hline 9,103 & $\mathrm{UA} / \mathrm{NA}$ & UA/NA & UA/NA & UA/NA & HOWARD \\
\hline 27,218 & 3,361 & 23,837 & 20 & UA/NA & ILLINOIS, CHICAGO \\
\hline 9,428 & $\mathrm{UA} / \mathrm{NA}$ & 8,143 & 241 & 1,044 & ILLINOIS, URBANA \\
\hline 12,757 & 0 & 9,196 & 3,561 & UA/NA & INDIANA \\
\hline 17,253 & 0 & 13,737 & 3,516 & UA/NA & IOWA \\
\hline 32,726 & 0 & 23,925 & 2,777 & 6,024 & IOWA STATE \\
\hline 38,476 & $\mathrm{UA} / \mathrm{NA}$ & 3,080 & 129 & 35,267 & JOHNS HOPKINS \\
\hline 15,939 & 370 & 5,457 & 2,616 & 7,496 & KANSAS \\
\hline 21,420 & UA/NA & 5,784 & 417 & 15,219 & KENT STATE \\
\hline 34,464 & 1,501 & 8,207 & 872 & 23,884 & KENTUCKY \\
\hline 10,331 & UA/NA & 4,636 & 2,558 & 3,137 & LAVAL \\
\hline 6,653 & UA/NA & 4,980 & 703 & 970 & LOUISIANA STATE \\
\hline 550 & 550 & UA/NA & UA/NA & UA/NA & LOUISVILLE \\
\hline 15,659 & 0 & 12,896 & 2,763 & 0 & MCGILL \\
\hline 30,055 & 0 & 29,136 & 144 & 775 & MCMASTER \\
\hline 26,094 & 0 & 23,765 & 2,329 & 0 & MANITOBA \\
\hline 28,950 & 3,639 & 24,000 & 55 & 1,256 & MARYLAND \\
\hline 15,893 & 6,813 & 1,854 & 507 & 6,719 & MASSACHUSETTS \\
\hline 18,109 & 0 & 12,320 & 785 & 5,004 & MIT \\
\hline 5,646 & 0 & 5,338 & 293 & 15 & MIAMI \\
\hline UA/NA & UA/NA & UA/NA & UA/NA & UA/NA & MICHIGAN \\
\hline 31,567 & 12,071 & 10,346 & 1,794 & 7,356 & MICHIGAN STATE \\
\hline 20,263 & UA/NA & UA/NA & UA/NA & UA/NA & MINNESOTA \\
\hline 21,686 & 14,767 & 5,369 & 991 & 559 & MISSOURI \\
\hline 7,375 & 0 & 10,620 & 4,303 & 0 & MONTREAL \\
\hline 7,288 & 0 & 0 & 2,003 & 5,285 & NEBRASKA \\
\hline 18,177 & 2,553 & 8,132 & 384 & 7,108 & NEW MEXICO \\
\hline 33,266 & UA/NA & 22,650 & 863 & 9,753 & NEW YORK \\
\hline 29,041 & 6,338 & 3,694 & 8,920 & 10,089 & NORTH CAROLINA \\
\hline 30,902 & 15,142 & 6,180 & 928 & 8,652 & NORTH CAROLINA STATE \\
\hline 30,093 & $\mathrm{UA} / \mathrm{NA}$ & UA/NA & UA/NA & $\mathrm{UA} / \mathrm{NA}$ & NORTHWESTERN \\
\hline 22,335 & UA/NA & UA/NA & UA/NA & UA/NA & NOTRE DAME \\
\hline 17,279 & 452 & 4,580 & 119 & 12,128 & $\mathrm{OHIO}$ \\
\hline 10,405 & 2,868 & 3,485 & 2,613 & 1,439 & OHIO STATE \\
\hline 11,818 & 1,080 & 3,737 & 68 & 6,933 & OKLAHOMA \\
\hline 15,131 & 4,389 & 10,432 & UA/NA & 310 & OKLAHOMA STATE \\
\hline 23,458 & 2,660 & 17,960 & 1,290 & 1,548 & OREGON \\
\hline 9,957 & UA/NA & 9,139 & 40 & 778 & PENNSYLVANIA \\
\hline
\end{tabular}




\section{Collections: Current Serials} $\begin{array}{rrr}\text { Current Serials } & \text { Current Serials } & \text { Current Serials } \\ \text { Total } & \text { Purchased } & \text { Purchased }\end{array}$

Electronic

Current Serials Purchased Print

(Survey Question Number)

(5)

(5a)

(5a.i)

(5a.ii)
INSTITUTION

PENNSYLVANIA STATE

PITTSBURGH

PRINCETON

PURDUE

QUEEN'S

RICE

ROCHESTER

RUTGERS

SASKATCHEWAN

SOUTH CAROLINA

SOUTHERN CALIFORNIA

SOUTHERN ILLINOIS

SUNY-ALBANY

SUNY-BUFFALO

SUNY-STONY BROOK

SYRACUSE

TEMPLE

TENNESSEE

TEXAS

TEXAS A\&M

TEXAS TECH

TORONTO

TULANE

UTAH

VANDERBILT

VIRGINIA

VIRGINIA TECH

WASHINGTON

WASHINGTON STATE

WASHINGTON U.-ST. LOUIS

WATERLOO

WAYNE STATE

WESTERN ONTARIO

WISCONSIN

YALE

YORK

BOSTON PUBLIC

CENTER FOR RESEARCH LIBRARIES

LIBRARY OF CONGRESS

Notes

\begin{tabular}{|c|c|c|c|c|}
\hline BbGLM+ & 99,091 & 68,556 & 55,600 & 12,956 \\
\hline BGLM+ & 87,251 & 78,691 & 72,341 & 6,350 \\
\hline bG+ & 60,216 & 58,045 & 47,381 & 10,664 \\
\hline G+ & 48,283 & 34,785 & 31,368 & 3,417 \\
\hline LM+ & 69,981 & 52,667 & 44,758 & 7,909 \\
\hline G+ & 81,123 & 61,821 & 57,940 & 3,881 \\
\hline $\mathrm{bM}+$ & 36,033 & 28,810 & 25,699 & 3,111 \\
\hline BGL+ & 68,560 & 42,783 & 35,890 & 6,893 \\
\hline GLM+ & 40,981 & 28,223 & 26,433 & 1,790 \\
\hline LM+ & 66,309 & 14,384 & 9,587 & 4,797 \\
\hline BGLM+ & 98,728 & 76,335 & 65,720 & 10,615 \\
\hline LM+ & 51,350 & 36,938 & 32,585 & 4,353 \\
\hline G+ & 50,693 & 36,345 & 24,917 & 11,428 \\
\hline GLM+ & 79,544 & 60,526 & 51,807 & 8,719 \\
\hline $\mathrm{bM}+$ & 95,517 & 80,421 & 74,473 & 5,948 \\
\hline GL+ & 39,703 & 27,637 & 24,572 & 3,065 \\
\hline BGLM+ & 62,173 & 46,070 & 42,233 & 3,837 \\
\hline BbGLM+ & 58,377 & 39,913 & 35,712 & 4,201 \\
\hline GL+ & 97,206 & 68,073 & 47,922 & 20,151 \\
\hline $\mathrm{BbG}+$ & 91,580 & 60,339 & 53,909 & 6,430 \\
\hline BLM+ & 84,317 & 59,895 & 55,754 & 4,141 \\
\hline BLM+ & 82,413 & 73,909 & 60,559 & 13,350 \\
\hline BGLM+ & 83,452 & 54,864 & 45,789 & 9,075 \\
\hline LM+ & 71,926 & 31,280 & 20,230 & 11,050 \\
\hline GLM+ & 55,260 & 30,837 & 23,446 & 7,391 \\
\hline LM+ & 117,749 & UA/NA & UA/NA & UA/NA \\
\hline G+ & 27,150 & 13,360 & 12,241 & 1,119 \\
\hline BGLM+ & 63,575 & 41,990 & 30,832 & 11,158 \\
\hline $\mathrm{BbG}+$ & 35,609 & UA/NA & UA/NA & $\mathrm{UA} / \mathrm{NA}$ \\
\hline GLM+ & 71,905 & 55,097 & 49,157 & 5,940 \\
\hline G+ & 35,054 & 16,138 & 11,841 & 4,297 \\
\hline GLM+ & 56,616 & 44,239 & 41,078 & 3,161 \\
\hline GL+ & 70,023 & 62,260 & 56,318 & 5,942 \\
\hline GLM+ & 101,596 & 69,757 & 49,807 & 19,950 \\
\hline GLM+ & 96,721 & 86,846 & 63,666 & 23,180 \\
\hline BGL+ & 75,381 & 67,266 & 59,874 & 7,392 \\
\hline \multirow[t]{2}{*}{ bG+ } & 4,984 & 4,630 & 167 & 4,463 \\
\hline & UA/NA & UA/NA & UA/NA & $\mathrm{UA} / \mathrm{NA}$ \\
\hline bG+ & 215,845 & 103,845 & 44,108 & 59,737 \\
\hline
\end{tabular}




\section{Collections: Current Serials}

Current Serials Current Serials

Not Purchased Not Purchased Consortial

(5b)
Current Serials

Not Purchased

Freely Accessible
Current Serials

Not Purchased

Print

(5b.iii)
Current Serials

Not Purchased GovDocs

(5b.iv) (Survey Question Number)

INSTITUTION

\begin{tabular}{|c|c|c|c|c|c|}
\hline 30,535 & 1,005 & 23,007 & 1,314 & 5,209 & PENNSYLVANIA STATE \\
\hline 8,560 & 0 & 6,866 & 1,297 & 397 & PITTSBURGH \\
\hline 2,171 & UA/NA & 1,000 & 29 & 1,142 & PRINCETON \\
\hline 13,498 & UA/NA & UA/NA & UA/NA & UA/NA & PURDUE \\
\hline 17,314 & $\mathrm{UA} / \mathrm{NA}$ & 16,611 & 703 & UA/NA & QUEEN'S \\
\hline 19,302 & 0 & 13,174 & 209 & 5,919 & RICE \\
\hline 7,223 & 0 & 6,366 & 857 & UA/NA & ROCHESTER \\
\hline 25,777 & 7,394 & 9,340 & 425 & 8,618 & RUTGERS \\
\hline 12,758 & 0 & 9,418 & 204 & 3,136 & SASKATCHEWAN \\
\hline 51,925 & 50,178 & 1,025 & 665 & 57 & SOUTH CAROLINA \\
\hline 22,393 & 170 & 19,058 & 3,165 & 0 & SOUTHERN CALIFORNIA \\
\hline 14,412 & 0 & 14,027 & 385 & UA/NA & SOUTHERN ILLINOIS \\
\hline 14,348 & 6,898 & 4,790 & 0 & 2,660 & SUNY-ALBANY \\
\hline 19,018 & 0 & 18,319 & 248 & 451 & SUNY-BUFFALC \\
\hline 15,096 & 7,791 & 7,203 & 102 & 0 & SUNY-STONY BROOK \\
\hline 12,066 & UA/NA & 6,715 & 1,647 & 3,704 & SYRACUSE \\
\hline 16,103 & 0 & 14,828 & 375 & 900 & TEMPLE \\
\hline 18,464 & 4,913 & 3,898 & 9 & 9,644 & TENNESSEE \\
\hline 29,133 & UA/NA & 21,911 & 3,290 & 3,932 & TEXAS \\
\hline 31,241 & 11,284 & 8,896 & 603 & 10,458 & TEXAS A\&M \\
\hline 24,422 & 0 & 24,254 & 106 & 62 & TEXAS TECH \\
\hline 8,504 & UA/NA & 5,878 & 2,626 & UA/NA & TORONTC \\
\hline 28,588 & 0 & 28,539 & 49 & 0 & TULANE \\
\hline 40,646 & 32,376 & 4,510 & 2,933 & 827 & UTAH \\
\hline 24,423 & 8,845 & 10,438 & 581 & 4,559 & VANDERBILT \\
\hline $\mathrm{UA} / \mathrm{NA}$ & UA/NA & UA/NA & UA/NA & UA/NA & VIRGINIA \\
\hline 13,790 & 10,427 & 800 & 100 & 2,463 & VIRGINIA TECH \\
\hline 21,585 & UA/NA & 12,861 & 2,958 & 5,766 & WASHINGTON \\
\hline $\mathrm{UA} / \mathrm{NA}$ & $\mathrm{UA} / \mathrm{NA}$ & UA/NA & $\mathrm{UA} / \mathrm{NA}$ & UA/NA & WASHINGTON STATE \\
\hline 16,808 & 2,946 & 10,040 & 1,542 & 2,280 & WASHINGTON U.-ST. LOUIS \\
\hline 18,916 & UA/NA & 18,916 & UA/NA & UA/NA & WATERLOC \\
\hline 12,377 & 6,487 & 4,371 & 441 & 1,078 & WAYNE STATE \\
\hline 7,763 & 0 & 5,548 & 1,442 & 773 & WESTERN ONTARIC \\
\hline 31,839 & UA/NA & 26,572 & 4,419 & 848 & WISCONSIN \\
\hline 9,875 & 3,688 & 4,904 & 1,283 & 0 & YALE \\
\hline 8,115 & UA/NA & 5,990 & 758 & 1,367 & YORK \\
\hline 354 & UA/NA & UA/NA & UA/NA & 354 & BOSTON PUBLIC \\
\hline $\mathrm{UA} / \mathrm{NA}$ & $\mathrm{UA} / \mathrm{NA}$ & $\mathrm{UA} / \mathrm{NA}$ & $\mathrm{UA} / \mathrm{NA}$ & UA/NA & CENTER FOR RESEARCH LIBRARIES \\
\hline 112,000 & 4,000 & 8,000 & 100,000 & 0 & LIBRARY OF CONGRESS \\
\hline
\end{tabular}




\section{Collections: Current Serials}

Current Serials Total
Current Serials
Purchased

(5)

(5a)
Current Serials Purchased Electronic
Current Serials Purchased Print (5a.ii)

(Survey Question Number)

(5a.i)

$\begin{array}{rrr}3,367 & 1,281 & 2,086 \\ 1,936 & 266 & 1,670 \\ 18,356 & 4,679 & 13,677 \\ 73,881 & 25,761 & 48,120 \\ 29,719 & 22,131 & 7,588 \\ 3,645 & 523 & 3,122\end{array}$

+ - See Footnotes

L - Includes Law library

M- Includes Medical library

\author{
G - Government documents not included in serials count \\ $\mathrm{B}$ - Includes branch campuses \\ $\mathrm{b}$ - Basis of volume count is bibliographic
}

U/A - Unavailable

N/A - Not Applicable

- no data supplied

\section{Summary Data}

Current Serials Total

(Survey Question Number)

University Libraries

Mean
Median
High
Low
Totals
Number of Libraries Reporting
nuniversity Libraries
Median
Totals
Number of Libraries Reporting

GRAND TOTALS

(5)
Current Serials Purchased

Current Serials Purchased Electronic

Current Serials Purchased Print

74,053

73,763

144,787

24,307

$8,442,054$

114

18,945

391,495

8

$8,833,549$

$6,046,950$

$4,899,835$ (5a)

(5a.i)

(5a.ii)

8,148

5,942

49,949

791

871,884

107

107

2,980

6,026

98,916

140,463

239,379

8

8

8

2




\section{Collections: Current Serials}

Current Serials

Not Purchased

(5b)
Current Serials

Not Purchased Consortial
Current Serials

Not Purchased Freely Accessible
Current Serials

Not Purchased Print

(5b.iii)
Current Serials

Not Purchased

GovDocs

INSTITUTION

\begin{tabular}{rrrrrr}
\hline 12,534 & 0 & 2,791 & 9,743 & 0 & NATIONAL AGRICULTURAL LIBRARY \\
UA/NA & UA/NA & UA/NA & UA/NA & UA/NA & LIBRARY \& ARCHIVES CANADA \\
3,633 & UA/NA & 1,669 & 1,718 & 246 & NATIONAL LIBRARY OF MEDICINE \\
9,710 & UA/NA & UA/NA & UA/NA & UA/NA & NEW YORK PUBLIC \\
10,885 & UA/NA & UA/NA & 6,070 & 4,815 & NEW YORK STATE \\
3,000 & UA/NA & UA/NA & 2,713 & 287 & SMITHSONIAN
\end{tabular}

Current Serials Not Purchased
Current Serials Current Serials Not Not Purchased Consortial (5b.i)
Purchased Freely Accessible (5b.ii)

\section{Summary Data}

Current Serials Not Purchased Print (5b.iii)
Current Serials

Not Purchased GovDocs

(5b.iv)(Survey Question Number)

$\begin{array}{rrrrrr}20,157 & 5,064 & 10,795 & 1,759 & 5,007 & \text { Mean } \\ 18,690 & 1,291 & 8,138 & 758 & 2,562 & \text { Median } \\ 51,925 & 50,178 & 29,482 & 13,427 & 35,267 & \text { High } \\ 550 & 0 & 0 & 0 & 0 & \text { Low } \\ 2,176,971 & 384,867 & 1,079,528 & 174,180 & 450,652 & \text { Totals } \\ 108 & 76 & 100 & 99 & 90 & \text { Nonuniversity Libraries } \\ 9,710 & & & & 267 & \text { Median } \\ 152,116 & 2,000 & & & 5,702 & \text { Totals } \\ 7 & 4,000 & 12,460 & 120,244 & 6 & \text { Number of Libraries Reporting } \\ 2,329,087 & 2 & 3 & 5 & & 456,354\end{array}$




\section{Collections: Other Materials}

Microform Government Computer Manuscripts Cartographic Graphic Audio Film Units Documents Files and Archives Materials Materials Materials and Video (Survey Question Number)

(7)

(8)

(9)

(10)

(11)

(12)

(13)

(14)

INSTITUTION

\begin{tabular}{|c|c|c|c|c|c|c|c|c|c|}
\hline ALABAMA & bGLM+ & $4,102,551$ & 884,759 & 8,785 & 35,331 & 312,829 & 189,324 & 24,138 & 7,628 \\
\hline ALBERTA & GLM+ & $3,744,257$ & 0 & 9,410 & 22,932 & $1,547,692$ & UA/NA & UA/NA & $\mathrm{UA} / \mathrm{NA}$ \\
\hline ARIZONA & bGLM+ & $6,308,419$ & 0 & 21,456 & 10,949 & 270,518 & 359,043 & 45,225 & 29,855 \\
\hline ARIZONA STATE & BGL+ & $7,614,192$ & 667,651 & 7,624 & 25,640 & 235,976 & $1,276,003$ & 68,902 & 37,931 \\
\hline AUBURN & + & $2,718,945$ & 236,917 & 3,442 & 10,699 & 152,582 & 75,327 & 116,107 & 5,296 \\
\hline BOSTON & $\mathrm{LM}+$ & $4,746,454$ & UA/NA & 2,809 & 51,659 & 1,701 & UA/NA & 74,939 & 9,997 \\
\hline BOSTON COLLEGE & $\mathrm{L}+$ & $4,226,524$ & 219,174 & 6,530 & 8,277 & 17,994 & 127,742 & 23,125 & 25,221 \\
\hline BRIGHAM YOUNG & GL+ & $3,888,004$ & 420,117 & UA/NA & 46,321 & 259,583 & $1,487,636$ & 267,012 & 32,156 \\
\hline BRITISH COLUMBIA & LM+ & $5,324,239$ & 0 & 23,320 & 13,084 & 618,248 & $1,009,742$ & 87,427 & 40,847 \\
\hline BROWN & bGM+ & $1,972,983$ & 0 & 5,599 & 13,142 & 154,683 & 950,402 & 80,076 & 11,250 \\
\hline CALGARY & GLM+ & $3,665,502$ & 0 & UA/NA & 13,078 & $1,436,661$ & $1,143,246$ & 41,641 & 16,758 \\
\hline CALIFORNIA, BERKELEY & $\mathrm{L}+$ & $7,149,224$ & 465,913 & 22,811 & 88,185 & 444,417 & $9,764,032$ & 97,168 & 53,401 \\
\hline CALIFORNIA, DAVIS & GLM+ & $4,266,788$ & 73,387 & 13,358 & 18,691 & 263,845 & 234,506 & 12,769 & 5,464 \\
\hline CALIFORNIA, IRVINE & bGM+ & $2,913,560$ & 149,103 & 12,111 & 4,503 & 11,409 & 71,661 & 16,641 & 15,386 \\
\hline CALIFORNIA, LOS ANGELES & bGLM+ & $6,246,884$ & 74,779 & 17,205 & 70,590 & 639,644 & $4,143,108$ & 278,488 & 38,035 \\
\hline CALIFORNIA, RIVERSIDE & G+ & $2,323,133$ & 304,308 & 12,396 & 4,920 & 106,717 & 22,762 & 22,873 & 22,499 \\
\hline CALIFORNIA, SAN DIEGO & bGM+ & $3,339,220$ & 0 & 18,046 & 16,449 & 116,243 & 64,494 & 124,545 & 29,974 \\
\hline CALIFORNIA, SANTA BARBARA & G+ & $3,772,031$ & 448,265 & 13,374 & 17,392 & $5,740,377$ & 31,828 & 137,534 & 11,676 \\
\hline CASE WESTERN RESERVE & GLM+ & $2,622,179$ & 30,653 & 8,794 & 14,838 & 65,193 & 72,647 & 34,652 & 22,734 \\
\hline CHICAGO & bGLM+ & $3,147,928$ & UA/NA & 13,878 & 41,783 & 438,094 & 0 & 60,778 & 9,367 \\
\hline CINCINNATI & GLM+ & $3,376,630$ & 28,209 & 13,521 & 26,261 & 173,929 & 267,954 & 66,296 & 23,982 \\
\hline COLORADO & bG+ & $7,031,778$ & 955,045 & 12,531 & 38,977 & 219,518 & 981,524 & 71,020 & 17,854 \\
\hline COLORADO STATE & G+ & $1,158,352$ & UA/NA & 2,232 & 7,839 & 42,510 & 4,607 & 769 & 99 \\
\hline COLUMBIA & GLM+ & $6,379,196$ & 174,032 & 38,162 & 245,118 & 140,021 & 871,581 & 113,057 & 43,661 \\
\hline CONNECTICUT & BbGLM+ & $4,342,083$ & 0 & 4,340 & 27,955 & 233,354 & 2,420 & 61,639 & 12,296 \\
\hline CORNELL & BGL+ & $8,507,500$ & 0 & 8,682 & 66,813 & 253,400 & 53,000 & 103,944 & 41,079 \\
\hline DARTMOUTH & bGM+ & $2,586,962$ & UA/NA & 11,984 & 25,082 & 192,146 & 507,236 & 37,815 & 21,538 \\
\hline DELAWARE & G+ & $3,447,958$ & 454,050 & 371,185 & 5,319 & 126,732 & 781 & 1,988 & 20,631 \\
\hline DUKE & LM+ & $4,359,354$ & $1,258,224$ & 40,440 & 57,246 & 132,961 & 927,639 & 66,210 & 81,517 \\
\hline EMORY & BLM+ & $6,142,110$ & 244,737 & 12,199 & 23,808 & 17,526 & 2,959 & 52,728 & 41,633 \\
\hline FLORIDA & BbLM+ & $7,919,279$ & $1,246,243$ & 9,010 & 8,930 & 864,701 & 246,405 & 27,940 & 45,956 \\
\hline FLORIDA STATE & BbLM+ & $9,114,981$ & 804,237 & 12,166 & 6,103 & 173,352 & 1,269 & 61,856 & 19,833 \\
\hline GEORGE WASHINGTON & BGLM+ & $1,432,040$ & 3,500 & 1,140 & 3,323 & 75 & 3,812 & 2,222 & 2,190 \\
\hline GEORGETOWN & bGLM+ & $5,067,722$ & $\mathrm{UA} / \mathrm{NA}$ & 7,812 & 18,695 & 2,053 & 378,169 & 22,154 & 35,710 \\
\hline GEORGIA & GL+ & $6,650,817$ & $1,070,308$ & 22,971 & 60,192 & 660,801 & $1,676,555$ & 140,467 & 170,257 \\
\hline GEORGIA TECH & G+ & $4,655,138$ & 0 & 15,784 & 7,224 & 198,288 & 116,797 & 1,802 & 7,445 \\
\hline GUELPH & BG+ & $2,357,880$ & 0 & UA/NA & 14,173 & 75,326 & 6,888 & 12,542 & $\mathrm{UA} / \mathrm{NA}$ \\
\hline HARVARD & GLM+ & $9,997,130$ & $\mathrm{UA} / \mathrm{NA}$ & $\mathrm{UA} / \mathrm{NA}$ & $\mathrm{UA} / \mathrm{NA}$ & $\mathrm{UA} / \mathrm{NA}$ & $\mathrm{UA} / \mathrm{NA}$ & $\mathrm{UA} / \mathrm{NA}$ & $\mathrm{UA} / \mathrm{NA}$ \\
\hline HAWAII & GLM+ & $3,518,804$ & 0 & 5,466 & 7,997 & 93,988 & 3,119 & 36,094 & 36,57 \\
\hline
\end{tabular}




\section{Collections: Other Materials}

Microform Government Computer Manuscripts Cartographic Graphic Audio Film Units Documents Files and Archives Materials Materials Materials and Video

(Survey Question Number)

(7)

(8)

(9)

(10)

(11)

(12)

(13)

(14)

INSTITUTION

\begin{tabular}{|c|c|c|c|c|c|c|c|c|c|}
\hline HOUSTON & $\mathrm{bL}+$ & $5,989,294$ & 680,971 & 2,766 & 5,124 & 5,704 & 526 & 17,218 & 8,906 \\
\hline HOWARD & bLM+ & $4,262,206$ & 2,364 & 395 & 189,657 & 40 & 155,211 & 48,119 & 6,554 \\
\hline ILLINOIS, CHICAGO & $\mathrm{M}+$ & $4,197,939$ & 793,177 & UA/NA & 34,726 & 204,768 & $2,137,298$ & 6,474 & 9,550 \\
\hline ILLINOIS, URBANA & bGL+ & $10,667,033$ & 0 & 15,007 & 55,706 & 701,487 & 91,936 & 180,586 & 32,882 \\
\hline INDIANA & $\mathrm{L}+$ & $5,115,583$ & 311,112 & 81,487 & 55,779 & 528,461 & $3,053,862$ & 309,155 & 67,458 \\
\hline IOWA & $\mathrm{LM}+$ & UA/NA & UA/NA & UA/NA & 17,408 & 355,363 & UA/NA & 33,850 & 35,682 \\
\hline IOWA STATE & G+ & $3,540,812$ & 0 & 11,605 & 17,842 & 99,038 & 920,761 & 17,164 & 32,874 \\
\hline JOHNS HOPKINS & BbGM+ & $4,406,186$ & 0 & 6,172 & 16,106 & 217,136 & 69,342 & 35,252 & 12,736 \\
\hline KANSAS & BbGLM+ & $3,177,385$ & 741,660 & 13,996 & 56,668 & 337,784 & $3,409,138$ & 39,611 & 26,402 \\
\hline KENT STATE & $\mathrm{BbG}+$ & UA/NA & 0 & 5,038 & 11,614 & 272,350 & 27,106 & 17,757 & 14,806 \\
\hline KENTUCKY & GLM+ & $6,506,097$ & $1,036,135$ & 8,656 & 27,663 & 262,549 & $2,148,689$ & 52,766 & 43,951 \\
\hline LAVAL & GLM+ & $1,309,880$ & UA/NA & 7,890 & $\mathrm{UA} / \mathrm{NA}$ & 303,307 & 214,738 & 24,783 & 30,358 \\
\hline LOUISIANA STATE & GLM+ & $9,038,682$ & 588,683 & 9,857 & 23,935 & 483,512 & UA/NA & 23,207 & 4,102 \\
\hline LOUISVILLE & BGM+ & $2,223,459$ & $\mathrm{UA} / \mathrm{NA}$ & UA/NA & 19,401 & 22,340 & $1,514,744$ & 36,475 & 19,569 \\
\hline MCGILL & BbGLM+ & $1,796,335$ & 676,933 & 9,454 & 3,434 & 217,987 & 313,305 & 55,483 & 14,106 \\
\hline MCMASTER & bGM+ & $1,517,413$ & 0 & 2,588 & 13,081 & 139,524 & 253 & 28,867 & 2,235 \\
\hline MANITOBA & GLM+ & $1,474,658$ & 221,129 & 0 & 14,180 & 105,745 & 270,053 & 30,870 & 10,817 \\
\hline MARYLAND & G+ & $5,817,532$ & 200,367 & 7,920 & 43,086 & 223,712 & 425,827 & 355,434 & 50,133 \\
\hline MASSACHUSETTS & G+ & $2,587,145$ & 0 & 1,905 & 15,931 & 99,486 & 250,060 & 21,952 & 5,512 \\
\hline MIT & G+ & $2,420,042$ & 0 & 65,444 & 24,764 & 127,106 & 463,556 & 34,022 & 7,501 \\
\hline MIAMI & bGLM+ & $4,030,606$ & 0 & 3,956 & 18,112 & 60,843 & 12,762 & 64,791 & 15,310 \\
\hline MICHIGAN & GLM+ & $\mathrm{UA} / \mathrm{NA}$ & 0 & $\mathrm{UA} / \mathrm{NA}$ & 49,480 & 385,090 & $21,381,302$ & 61,653 & 44,399 \\
\hline MICHIGAN STATE & GL+ & $6,806,042$ & 0 & 27,796 & 2,850 & 234,226 & 1,817 & 69,223 & 6,852 \\
\hline MINNESOTA & bGLM+ & $7,028,582$ & $2,641,702$ & 18,072 & 81,526 & 475,365 & $\mathrm{UA} / \mathrm{NA}$ & UA/NA & $\mathrm{UA} / \mathrm{NA}$ \\
\hline MISSOURI & GLM+ & $8,142,874$ & $1,684,682$ & 2,401 & 12,234 & 278,849 & 630 & 26,246 & 6,277 \\
\hline MONTREAL & GLM+ & $1,558,155$ & 0 & 7,629 & 0 & 13,174 & 117,098 & 65,295 & 16,246 \\
\hline NEBRASKA & $\mathrm{L}+$ & $4,704,552$ & 552,672 & 8,754 & 10,870 & 169,698 & 103,832 & 37,925 & 4,835 \\
\hline NEW MEXICO & GLM+ & $4,723,386$ & 666,566 & 87,856 & 16,335 & 234,581 & $1,096,206$ & 37,544 & 10,856 \\
\hline NEW YORK & bGLM+ & $5,592,987$ & 483,140 & 6,221 & 37,294 & 441 & $2,423,749$ & 113,928 & 51,217 \\
\hline NORTH CAROLINA & GLM+ & $5,179,705$ & $1,625,932$ & 13,333 & 40,726 & 294,238 & $1,631,367$ & 338,251 & 69,422 \\
\hline NORTH CAROLINA STATE & G+ & $5,447,358$ & 0 & 421,287 & 14,591 & 52,070 & 133,271 & 4,738 & 26,410 \\
\hline NORTHWESTERN & BGLM+ & $4,603,214$ & 7,808 & 17,014 & 18,069 & 208,757 & 601,153 & 66,977 & 33,882 \\
\hline NOTRE DAME & bGL+ & $4,014,686$ & $\mathrm{UA} / \mathrm{NA}$ & 6,722 & 35,234 & 20,850 & 230,008 & 29,357 & 11,676 \\
\hline OHIO & BbGM+ & $1,999,023$ & 0 & 4,271 & 7,300 & 45,522 & 182,198 & 36,711 & 38,004 \\
\hline OHIO STATE & bGLM+ & $6,111,550$ & 884 & 23,916 & 36,191 & 231,350 & $2,481,419$ & 84,840 & $\mathrm{UA} / \mathrm{NA}$ \\
\hline OKLAHOMA & GLM+ & $4,476,140$ & 41,455 & 5,756 & 18,509 & 155,967 & $1,750,069$ & 4,908 & 8,721 \\
\hline OKLAHOMA STATE & BGM+ & 455,806 & 189,681 & 16,127 & 11,091 & 321,020 & 85,523 & 14,048 & 19,688 \\
\hline OREGON & BGL+ & $4,162,266$ & 248,790 & 3,023 & 74,656 & 781,668 & $1,326,973$ & 78,364 & 30,647 \\
\hline PENNSYLVANIA & GLM+ & $4,168,493$ & $\mathrm{UA} / \mathrm{NA}$ & UA/NA & 16,000 & 139,201 & 805,535 & 93,375 & 22,532 \\
\hline
\end{tabular}




\section{Collections: Other Materials}

Microform Government Computer Manuscripts Cartographic Graphic Audio Film

Documents Files and Archives
(Survey Question Number)
(7)
(8)
(9)
(10)
(11)
(12)
(13)
(14)

INSTITUTION

\begin{tabular}{|c|c|c|c|c|c|c|c|c|c|}
\hline PENNSYLVANIA STATE & BbGLM+ & $5,310,696$ & 0 & 11,341 & 32,830 & 417,434 & 613,404 & 82,852 & 108,399 \\
\hline PITTSBURGH & BGLM+ & $5,428,310$ & 0 & 74,203 & 31,828 & 124,717 & 812,657 & 115,944 & 97,51 \\
\hline PRINCETON & bG+ & $6,612,465$ & 3,071 & 12,003 & 37,840 & 344,394 & 250,904 & 96,365 & 11,05 \\
\hline PURDUE & G+ & $3,102,402$ & 484,234 & 13,576 & 3,960 & 344,738 & 1,511 & 145 & 11,607 \\
\hline QUEEN'S & $\mathrm{LM}+$ & $4,059,040$ & $1,120,094$ & $\mathrm{UA} / \mathrm{NA}$ & $\mathrm{UA} / \mathrm{NA}$ & 159,859 & 28,568 & UA/NA & $\mathrm{UA} / \mathrm{NA}$ \\
\hline RICE & G+ & $3,328,017$ & 55,896 & 16,039 & 8,253 & 27,167 & 34,900 & 51,216 & 14,687 \\
\hline ROCHESTER & $\mathrm{bM}+$ & $5,144,222$ & 419,912 & 7,537 & 15,630 & 105,426 & 11,814 & 101,376 & 23,661 \\
\hline RUTGERS & BGL+ & $6,065,247$ & $2,671,041$ & 15,925 & 28,430 & $\mathrm{UA} / \mathrm{NA}$ & $\mathrm{UA} / \mathrm{NA}$ & UA/NA & $\mathrm{UA} / \mathrm{NA}$ \\
\hline SASKATCHEWAN & GLM+ & $3,086,526$ & 415,902 & 3,933 & 268 & 74,022 & 18,714 & 17,825 & 1,936 \\
\hline SOUTH CAROLINA & $\mathrm{LM}+$ & $5,394,547$ & 951,954 & 5,793 & 11,449 & 325,818 & 250,294 & 39,000 & 17,805 \\
\hline SOUTHERN CALIFORNIA & BGLM+ & $6,415,794$ & 273,598 & 3,532 & 48,518 & 161,718 & $3,192,513$ & 34,487 & 36,182 \\
\hline SOUTHERN ILLINOIS & $\mathrm{LM}+$ & $4,617,239$ & 312,491 & 11,553 & 27,210 & 348,540 & 2,256 & 30,205 & 19,569 \\
\hline SUNY-ALBANY & G+ & $2,914,518$ & 0 & 2,360 & 12,863 & 25,687 & 64,565 & 8,574 & 9,514 \\
\hline SUNY-BUFFALO & GLM+ & $6,150,294$ & 0 & 14,848 & 12,703 & 199,101 & 105,511 & 61,942 & 19,546 \\
\hline SUNY-STONY BROOK & $\mathrm{bM}+$ & $3,848,942$ & 348,588 & 828 & 8,490 & 133,710 & 9,457 & 44,238 & 8,603 \\
\hline SYRACUSE & GL+ & $7,530,901$ & 293,267 & 2,020 & 30,420 & 280,743 & 417,766 & 415,940 & 23,522 \\
\hline TEMPLE & BGLM+ & $3,327,005$ & 24,883 & 3,675 & 43,294 & 121,694 & $10,038,653$ & 25,536 & 12,320 \\
\hline TENNESSEE & BbGLM+ & $4,391,748$ & 4,116 & 3,602 & 14,316 & 392,599 & 13,808 & 26,522 & 18,841 \\
\hline TEXAS & GL+ & $6,663,065$ & 0 & 12,524 & 127,536 & 331,882 & $12,580,591$ & 207,021 & 83,772 \\
\hline TEXAS A\&M & $\mathrm{BbG+}$ & $5,678,256$ & 65,519 & 11,248 & 12,185 & 237,235 & 541,905 & 27,388 & 37,558 \\
\hline TEXAS TECH & BLM+ & $2,760,234$ & 4,222 & 392,511 & 35,324 & 165,736 & $1,746,668$ & 26,393 & 37,941 \\
\hline TORONTO & BLM+ & $5,486,050$ & $\mathrm{UA} / \mathrm{NA}$ & 927,783 & 42,735 & 297,635 & 810,635 & 209,609 & 39,175 \\
\hline TULANE & BGLM+ & $2,267,026$ & 85,069 & 3,440 & 48,785 & 29,987 & 562 & 25,022 & 8,504 \\
\hline UTAH & $\mathrm{LM}+$ & $3,641,610$ & 898,005 & 8,680 & 37,248 & 254,001 & $1,701,690$ & 52,561 & 36,002 \\
\hline VANDERBILT & GLM+ & $3,112,303$ & 266,061 & 10,444 & 21,974 & 108,787 & 223,050 & 39,020 & 22,453 \\
\hline VIRGINIA & $\mathrm{LM}+$ & $5,442,787$ & 669,424 & 11,948 & 28,133 & 153,289 & 447,913 & 85,227 & 41,573 \\
\hline VIRGINIA TECH & $\mathrm{G}+$ & $6,329,176$ & 399,525 & 10,772 & 18,123 & 140,525 & 165,508 & 12,975 & 18,185 \\
\hline WASHINGTON & BGLM+ & $7,835,346$ & . & 19,500 & 69,041 & 427,762 & $1,353,536$ & 82,575 & 53,750 \\
\hline WASHINGTON STATE & $\mathrm{BbG+}$ & $3,948,577$ & 0 & 549 & 19,628 & 2,518 & 369,424 & 16,602 & 36,029 \\
\hline WASHINGTON U.-ST. LOUIS & GLM+ & $3,473,141$ & 0 & 5,327 & 19,056 & 117,862 & 3 & 43,993 & 62,592 \\
\hline WATERLOO & G+ & $1,712,333$ & 0 & 9,978 & 5,853 & 146,808 & 41 & 429 & 308 \\
\hline WAYNE STATE & GLM+ & $3,898,391$ & 388,182 & 5,915 & 70,500 & 56,345 & $2,161,039$ & 48,440 & 31,523 \\
\hline WESTERN ONTARIO & GL+ & $4,074,250$ & 0 & 0 & 13,976 & 15,381 & $1,600,000$ & 59,785 & 2,599 \\
\hline WISCONSIN & GLM+ & $6,412,542$ & 757,007 & 16,854 & 157,559 & 602,892 & $6,286,264$ & 358,219 & 32,209 \\
\hline YALE & GLM+ & $10,231,747$ & 0 & 0 & 96,927 & 465,001 & $2,393,832$ & 249,747 & 32,724 \\
\hline YORK & BGL+ & $4,225,417$ & $\mathrm{UA} / \mathrm{NA}$ & 6,163 & 12,673 & 112,192 & 8,647 & 38,427 & 26,580 \\
\hline BOSTON PUBLIC & bG+ & $7,009,486$ & $1,892,423$ & UA/NA & UA/NA & UA/NA & UA/NA & 40,334 & 115,918 \\
\hline CENTER FOR RESEARCH LIBRARIES & & $1,929,683$ & & 1,998 & & $\mathrm{UA} / \mathrm{NA}$ & $\mathrm{UA} / \mathrm{NA}$ & $\mathrm{UA} / \mathrm{NA}$ & $\mathrm{UA} / \mathrm{NA}$ \\
\hline LIBRARY OF CONGRESS & bG+ & $16,206,259$ & 84,146 & 884,601 & 63,718 & $5,391,200$ & $14,509,245$ & $3,052,857$ & $1,213,180$ \\
\hline
\end{tabular}




\section{Collections: Other Materials}

\begin{tabular}{|c|c|c|c|c|c|c|c|c|c|}
\hline & & $\begin{array}{l}\text { Microform } \\
\text { Units }\end{array}$ & $\begin{array}{c}\text { Government } \\
\text { Documents }\end{array}$ & $\begin{array}{r}\text { Computer } \\
\text { Files }\end{array}$ & $\begin{array}{l}\text { Manuscripts } \\
\text { and Archives }\end{array}$ & $\begin{array}{r}\text { Cartographic } \\
\text { Materials }\end{array}$ & $\begin{array}{r}\text { Graphic } \\
\text { Materials }\end{array}$ & $\begin{array}{r}\text { Audio } \\
\text { Materials }\end{array}$ & $\begin{array}{r}\text { Film } \\
\text { and Video }\end{array}$ \\
\hline (Survey Questior & n Number) & (7) & (8) & (9) & (10) & (11) & (12) & (13) & (14) \\
\hline & Notes & & & & & & & & \\
\hline TURAL LIBRARY & $\mathrm{G}^{+}$ & $1,100,928$ & 0 & 1,975 & 19,875 & 5,158 & 1,478 & 403 & 4,130 \\
\hline JES CANADA & G+ & $8,182,907$ & $334,014,813$ & 84 & 121,860 & $2,967,887$ & $25,548,173$ & 335,358 & 204,801 \\
\hline Y OF MEDICINE & G+ & 605,802 & $\cdot$ & 2,560 & 6,277 & 0 & 69,285 & $\cdot$ & 84,831 \\
\hline & + & $6,938,824$ & UA/NA & UA/NA & 65,995 & 433,915 & $4,405,581$ & 582,369 & 73,386 \\
\hline & G+ & $8,638,262$ & UA/NA & 9,092 & 29,467 & 212,142 & 72,015 & 59 & 101 \\
\hline & $\mathrm{G}+$ & 190,440 & 52,752 & 2,035 & 2,124 & 158 & 2,039 & 359 & 635 \\
\hline
\end{tabular}

+ - See Footnotes

L - Includes Law library

M- Includes Medical library
G - Government documents not included in serials count

B - Includes branch campuses

$\mathrm{b}-$ Basis of volume count is bibliographic
U/A - Unavailable

N/A - Not Applicable

. - no data supplied

\section{Summary Data}

Microform Units

(Survey Question Number)
Government Computer

Documents Files
Manuscripts Cartographic and Archives Materials
Graphic Audio Film

Materials Materials and Video

(12)

(13)

(14)

\section{University Libraries}

Mean
Median
High
Low
Totals
Number of Libraries Reporting

$4,642,450$
$4,266,788$
$10,667,033$
455,806
$515,312,001$
111

$\begin{array}{rr}345,215 & 32,609 \\ 117,086 & 9,656 \\ 2,671,041 & 927,783 \\ 0 & 0 \\ 34,521,515 & 3,391,291 \\ 100 & 104\end{array}$

$\begin{array}{rrrrr}31,822 & 290,166 & 1,169,777 & 71,811 & 27,476 \\ 18,695 & 183,038 & 250,060 & 43,993 & 22,453 \\ 245,118 & 5,740,377 & 21,381,302 & 415,940 & 170,257 \\ 0 & 40 & 0 & 145 & 308 \\ 3,532,249 & 32,498,601 & 125,166,138 & 7,827,402 & 2,939,972 \\ 111 & 112 & 107 & 109 & 107\end{array}$

\section{Nonuniversity Libraries}

Median

Totals

Number of Libraries Reporting

$\begin{array}{rrrrrrrr}6,938,824 & 84,146 & 2,035 & 29,467 & 212,142 & 72,015 & 40,334 & 79,109 \\ 50,802,591 & 336,044,134 & 902,345 & 309,316 & 9,010,460 & 44,607,816 & 4,011,739 & 1,696,982 \\ 9 & 5 & 7 & 7 & 7 & 7 & 7 & 8 \\ & & & & & & & \\ 566,114,592 & 370,565,649 & 4,293,636 & 3,841,565 & 41,509,061 & 169,773,954 & 11,839,141 & 4,636,954\end{array}$




\section{EXPENDITURES}

Monographs Current Serials Other Library Miscellaneous Total Library Contract Materials Materials Materials Binding

(Survey Question Number)

(16a)

(16b)

(16c)

(16d)

(16)

(17)

\begin{tabular}{|c|c|c|c|c|c|c|c|}
\hline INSTITUTION & Notes & & & & & & \\
\hline ALABAMA & bGLM+ & $1,114,921$ & $6,764,436$ & 290,942 & 36,767 & $8,207,066$ & 47,855 \\
\hline ALBERTA & GLM+ & $5,906,026$ & $10,050,328$ & 0 & 805,003 & $16,761,357$ & 62,079 \\
\hline ARIZONA & bGLM+ & $2,420,279$ & $8,980,989$ & 724,170 & 765,134 & $12,890,572$ & 33,390 \\
\hline ARIZONA STATE & BGL+ & $1,176,474$ & $8,962,161$ & 877,458 & 188,756 & $11,204,849$ & 48,404 \\
\hline AUBURN & + & 518,171 & $5,186,086$ & 55,994 & 0 & $5,760,251$ & 53,341 \\
\hline BOSTON & $\mathrm{LM}+$ & 894,004 & $8,275,790$ & 176,413 & 29,129 & $9,375,336$ & 142,720 \\
\hline BOSTON COLLEGE & $\mathrm{L}+$ & $1,472,388$ & $5,231,355$ & 868,320 & $1,589,455$ & $9,161,518$ & 77,290 \\
\hline BRIGHAM YOUNG & GL+ & $2,505,763$ & $6,890,140$ & 6,280 & 140,607 & $9,542,790$ & 124,439 \\
\hline BRITISH COLUMBIA & $\mathrm{LM}+$ & $3,648,620$ & $11,092,400$ & 0 & 625,584 & $15,366,604$ & 162,060 \\
\hline BROWN & bGM+ & $1,710,759$ & $5,936,815$ & 479,325 & 690,946 & $8,817,845$ & 60,763 \\
\hline CALGARY & GLM+ & $3,031,863$ & $7,402,153$ & 540,592 & 814,142 & $11,788,750$ & 74,802 \\
\hline CALIFORNIA, BERKELEY & $\mathrm{L}+$ & $6,357,361$ & $10,540,298$ & 893,363 & 58,368 & $17,849,390$ & $1,061,257$ \\
\hline CALIFORNIA, DAVIS & GLM+ & $1,981,284$ & $6,560,659$ & 175,905 & $\mathrm{UA} / \mathrm{NA}$ & $8,717,848$ & 131,500 \\
\hline CALIFORNIA, IRVINE & bGM+ & $2,152,745$ & $7,084,869$ & 251,892 & . & $9,489,506$ & 49,861 \\
\hline CALIFORNIA, LOS ANGELES & bGLM+ & $5,861,511$ & $8,099,419$ & UA/NA & 498,105 & $14,459,035$ & 373,261 \\
\hline CALIFORNIA, RIVERSIDE & $\mathrm{G}^{+}$ & 556,349 & $4,230,726$ & 15,695 & 0 & $4,802,770$ & 39,439 \\
\hline CALIFORNIA, SAN DIEGO & bGM+ & $3,015,924$ & $6,764,591$ & 95,718 & 0 & $9,876,233$ & 217,207 \\
\hline CALIFORNIA, SANTA BARBARA & G+ & 989,473 & $4,217,070$ & 243,097 & 0 & $5,449,640$ & 126,148 \\
\hline CASE WESTERN RESERVE & GLM+ & 694,410 & $5,831,618$ & 81,872 & 99,214 & $6,707,114$ & 47,706 \\
\hline CHICAGO & bGLM+ & $6,091,102$ & $11,982,017$ & 0 & 0 & $18,073,119$ & 336,557 \\
\hline CINCINNATI & GLM+ & $2,275,243$ & $7,192,136$ & 618,999 & 92,862 & $10,179,240$ & 135,303 \\
\hline COLORADO & $\mathrm{bG}+$ & $2,216,558$ & $7,430,771$ & 774,144 & 972,629 & $11,394,102$ & 70,095 \\
\hline COLORADO STATE & G+ & $1,055,691$ & $5,072,641$ & 14,841 & 366,216 & $6,509,389$ & 36,986 \\
\hline COLUMBIA & GLM+ & $6,664,952$ & $15,015,672$ & $1,396,953$ & $1,478,330$ & $24,555,907$ & 713,210 \\
\hline CONNECTICUT & BbGLM+ & $1,117,334$ & $7,910,971$ & 482,357 & 647,762 & $10,158,424$ & 155,680 \\
\hline CORNELL & BGL+ & $6,059,153$ & $8,472,429$ & 561,684 & $1,067,783$ & $16,161,049$ & 153,512 \\
\hline DARTMOUTH & bGM+ & $1,705,162$ & $7,567,386$ & 126,748 & UA/NA & $9,399,296$ & 63,310 \\
\hline DELAWARE & G+ & $2,302,481$ & $5,896,667$ & 497,916 & 272,108 & $8,969,172$ & 52,271 \\
\hline DUKE & $\mathrm{LM}+$ & $4,320,632$ & $10,453,502$ & 138,826 & $1,538,505$ & $16,451,465$ & 184,250 \\
\hline EMORY & BLM+ & $3,697,214$ & $6,694,006$ & $5,935,977$ & 650,560 & $16,977,757$ & 107,305 \\
\hline FLORIDA & $\mathrm{BbLM}+$ & $1,398,497$ & $9,380,631$ & 834,265 & 648,556 & $12,261,949$ & 101,792 \\
\hline FLORIDA STATE & BbLM+ & 934,853 & $6,007,992$ & 982,353 & 137,637 & $8,062,835$ & 48,989 \\
\hline GEORGE WASHINGTON & BGLM+ & $2,469,176$ & $7,101,214$ & 72,839 & $1,532,047$ & $11,175,276$ & 121,342 \\
\hline GEORGETOWN & bGLM+ & $2,494,661$ & $8,009,661$ & 945,832 & 560,179 & $12,010,333$ & 111,913 \\
\hline GEORGIA & GL+ & $1,811,698$ & $7,969,205$ & 182,109 & 401,766 & $10,364,778$ & 266,435 \\
\hline GEORGIA TECH & $\mathrm{G}^{+}$ & 276,824 & $4,571,221$ & $1,189,106$ & 0 & $6,037,151$ & 7,888 \\
\hline GUELPH & BG+ & $1,160,317$ & $3,681,947$ & 158,136 & 818,179 & $5,818,580$ & 23,866 \\
\hline HARVARD & GLM+ & $13,064,991$ & $8,448,811$ & $13,756,710$ & 25,971 & $35,296,483$ & $1,023,422$ \\
\hline HAWAII & GLM+ & $1,145,314$ & $6,791,920$ & 96,677 & 71,696 & $8,105,607$ & 208,952 \\
\hline
\end{tabular}




\section{EXPENDITURES}

Salaries \& Wages Salaries \& Wages Salaries \& Wages Total Salaries Other Operating Total Library Professional Staff Support Staff Student Assistants \&Wages Expenditures Expenditures
(18a)
$(18 b)$
(18c)
(18)
(20)
(21) (Survey Question Number)

\begin{tabular}{|c|c|c|c|c|c|c|}
\hline & & & & & & INSTITUTION \\
\hline $4,157,507$ & $2,011,116$ & 693,566 & $6,862,189$ & $2,313,804$ & $17,430,914$ & ALABAMA \\
\hline $6,636,528$ & $9,086,668$ & $1,086,285$ & $16,809,481$ & $8,697,279$ & $42,330,195$ & ALBERTA \\
\hline $5,736,506$ & $5,099,563$ & $1,066,436$ & $11,902,505$ & $5,819,286$ & $30,645,753$ & ARIZONA \\
\hline $5,207,484$ & $5,465,761$ & 588,326 & $11,261,571$ & $2,125,271$ & $24,640,095$ & ARIZONA STATE \\
\hline $3,029,393$ & $1,672,861$ & 386,633 & $5,088,887$ & $1,373,732$ & $12,276,211$ & AUBURN \\
\hline $5,578,963$ & $3,439,157$ & 634,195 & $9,652,315$ & $2,315,914$ & $21,486,285$ & BOSTON \\
\hline $5,498,330$ & $2,835,375$ & 655,507 & $8,989,212$ & $1,409,680$ & $19,637,700$ & BOSTON COLLEGE \\
\hline $7,690,060$ & $2,007,017$ & $3,060,465$ & $12,757,542$ & $3,469,781$ & $25,894,552$ & BRIGHAM YOUNG \\
\hline $8,191,761$ & $6,477,209$ & 802,490 & $15,471,461$ & $3,925,473$ & $34,925,597$ & BRITISH COLUMBIA \\
\hline $5,020,630$ & $4,338,040$ & 484,366 & $9,843,036$ & $2,279,701$ & $21,001,345$ & BROWN \\
\hline $5,290,867$ & $6,689,247$ & 572,342 & $12,552,456$ & $1,099,731$ & $25,515,738$ & CALGARY \\
\hline $17,862,497$ & $8,575,213$ & $3,175,391$ & $29,613,101$ & $3,449,351$ & $51,973,099$ & CALIFORNIA, BERKELEY \\
\hline $4,594,867$ & $4,971,916$ & 505,954 & $10,072,737$ & $1,010,819$ & $19,932,904$ & CALIFORNIA, DAVIS \\
\hline $4,475,900$ & $5,068,462$ & 639,295 & $10,183,657$ & $2,224,888$ & $21,947,912$ & CALIFORNIA, IRVINE \\
\hline $12,788,538$ & $12,263,906$ & $2,842,953$ & $27,895,397$ & $7,477,952$ & $50,205,645$ & CALIFORNIA, LOS ANGELES \\
\hline $3,614,626$ & $3,246,165$ & 433,927 & $7,294,718$ & $1,258,350$ & $13,395,277$ & CALIFORNIA, RIVERSIDE \\
\hline $8,016,125$ & $7,176,313$ & $1,404,236$ & $16,596,674$ & $3,386,044$ & $30,076,158$ & CALIFORNIA, SAN DIEGO \\
\hline 4,191,093 & $5,970,075$ & 913,175 & $11,074,343$ & $2,547,301$ & $19,197,432$ & CALIFORNIA, SANTA BARBARA \\
\hline $3,734,511$ & $1,485,134$ & 580,731 & $5,800,376$ & $1,464,064$ & $14,019,260$ & CASE WESTERN RESERVE \\
\hline $5,111,645$ & $6,722,893$ & $1,481,916$ & $13,316,454$ & $3,920,017$ & $35,646,147$ & CHICAGO \\
\hline $5,059,307$ & $2,126,610$ & 705,267 & $7,891,184$ & $2,124,416$ & $20,330,143$ & CINCINNATI \\
\hline $3,220,786$ & $4,914,086$ & 826,175 & $8,961,047$ & $1,706,513$ & $22,131,757$ & COLORADO \\
\hline $3,365,933$ & $2,173,820$ & 650,289 & $6,190,042$ & $2,597,665$ & $15,334,082$ & COLORADO STATE \\
\hline $20,584,544$ & $8,067,829$ & $2,498,524$ & $31,150,897$ & $6,900,223$ & $63,320,237$ & COLUMBIA \\
\hline $9,013,542$ & $4,997,521$ & 777,137 & $14,788,200$ & $1,728,705$ & $26,831,009$ & CONNECTICUT \\
\hline $8,783,165$ & $13,828,122$ & 982,871 & $23,594,158$ & $6,638,001$ & $46,546,720$ & CORNELL \\
\hline $4,254,920$ & $4,001,774$ & 434,607 & $8,691,301$ & $1,548,655$ & $19,702,562$ & DARTMOUTH \\
\hline $4,320,952$ & $3,325,590$ & 365,920 & $8,012,462$ & $1,700,375$ & $18,734,280$ & DELAWARE \\
\hline $11,612,246$ & $5,081,288$ & 585,372 & $17,278,906$ & $4,099,020$ & $38,013,641$ & DUKE \\
\hline $7,498,891$ & $5,281,681$ & 721,374 & $13,501,946$ & $4,133,319$ & $34,720,327$ & EMORY \\
\hline $6,263,261$ & $6,957,726$ & 544,698 & $13,765,685$ & $2,017,776$ & $28,147,202$ & FLORIDA \\
\hline $3,690,101$ & $2,785,218$ & 667,566 & $7,142,885$ & 495,777 & $15,750,486$ & FLORIDA STATE \\
\hline $5,883,544$ & $4,540,376$ & 659,220 & $11,083,140$ & $4,112,144$ & $26,491,902$ & GEORGE WASHINGTON \\
\hline $7,050,648$ & $4,785,525$ & 864,411 & $12,700,584$ & $3,217,227$ & $28,040,057$ & GEORGETOWN \\
\hline $4,240,268$ & $5,418,479$ & 740,446 & $10,399,193$ & $1,771,984$ & $22,802,390$ & GEORGIA \\
\hline $2,873,647$ & $2,399,980$ & 129,930 & $5,403,557$ & $1,063,027$ & $12,511,623$ & GEORGIA TECH \\
\hline $2,887,152$ & $2,597,468$ & 222,662 & $5,707,282$ & 946,003 & $12,495,731$ & GUELPH \\
\hline $42,980,437$ & $20,781,546$ & $1,697,333$ & $65,459,316$ & $21,200,914$ & $122,980,135$ & HARVARD \\
\hline $6,140,980$ & $2,876,413$ & 978,567 & $9,995,960$ & 766,420 & $19,076,939$ & HAWAII \\
\hline
\end{tabular}




\section{EXPENDITURES}

Monographs Current Serials Other Library Miscellaneous Total Library Contract Materials Materials Materials Binding

(Survey Question Number)

(16a)

(16b)

(16c)

(16d)

(16)

(17)

\begin{tabular}{|c|c|c|c|c|c|c|c|}
\hline INSTITUTION & Notes & & & & & & \\
\hline HOUSTON & $\mathrm{bL}+$ & $1,562,782$ & $6,633,041$ & $1,147,319$ & 560,129 & $9,903,271$ & 105,471 \\
\hline HOWARD & bLM+ & 244,960 & $3,658,418$ & 169,632 & 0 & $4,073,010$ & 17,000 \\
\hline ILLINOIS, CHICAGO & $\mathrm{M}+$ & $1,334,871$ & $4,398,769$ & $4,694,046$ & 258,662 & $10,686,348$ & 32,165 \\
\hline ILLINOIS, URBANA & bGL+ & $2,839,435$ & $10,773,374$ & 363,239 & 553,506 & $14,529,554$ & 267,720 \\
\hline INDIANA & $\mathrm{L}+$ & $5,757,033$ & $8,278,469$ & 563,715 & 195,674 & $14,794,891$ & 153,597 \\
\hline IOWA & LM+ & $2,504,908$ & $10,782,240$ & 672,770 & 318,435 & $14,278,353$ & 160,345 \\
\hline IOWA STATE & G+ & $1,684,193$ & $10,215,830$ & 264,492 & 396,245 & $12,560,760$ & 105,592 \\
\hline JOHNS HOPKINS & $\mathrm{BbGM}+$ & $2,582,724$ & $7,368,569$ & $4,249,182$ & 371,902 & $14,572,377$ & 67,911 \\
\hline KANSAS & BbGLM+ & $2,056,586$ & $6,660,380$ & 385,031 & 519,892 & $9,621,889$ & 117,426 \\
\hline KENT STATE & $\mathrm{BbG}+$ & 758,152 & $3,445,792$ & UA/NA & 0 & $4,203,944$ & 72,693 \\
\hline KENTUCKY & GLM+ & $1,423,984$ & $6,827,718$ & $1,891,592$ & 625,984 & $10,769,278$ & 110,014 \\
\hline LAVAL & GLM+ & $2,189,590$ & $6,990,673$ & 141,840 & 310,695 & $9,632,798$ & 50,787 \\
\hline LOUISIANA STATE & GLM+ & 940,843 & $5,308,904$ & 73,495 & 0 & $6,323,242$ & 42,241 \\
\hline LOUISVILLE & BGM+ & 876,426 & $4,282,651$ & $4,328,598$ & 0 & $9,487,675$ & 119,826 \\
\hline MCGILL & BbGLM+ & $3,799,947$ & $8,269,481$ & 210,059 & 709,968 & $12,989,455$ & 173,785 \\
\hline MCMASTER & bGM+ & $1,183,460$ & $5,666,674$ & 181,770 & 949,601 & $7,981,505$ & 6,200 \\
\hline MANITOBA & GLM+ & $1,690,627$ & $5,638,030$ & 103,709 & 323,643 & $7,756,008$ & 130,758 \\
\hline MARYLAND & G+ & $1,539,240$ & $5,624,692$ & 202,497 & $2,133,043$ & $9,499,472$ & 96,005 \\
\hline MASSACHUSETTS & G+ & 796,144 & $5,226,388$ & 167,452 & 227,461 & $6,417,445$ & 69,300 \\
\hline MIT & G+ & $1,528,568$ & $7,176,222$ & 245,907 & 101,216 & $9,051,913$ & 201,999 \\
\hline MIAMI & bGLM+ & $2,283,175$ & $11,176,016$ & 12,860 & 222,484 & $13,694,535$ & 74,297 \\
\hline MICHIGAN & GLM+ & $5,543,022$ & $14,141,584$ & 3,108 & $2,877,304$ & $22,565,018$ & 379,441 \\
\hline MICHIGAN STATE & GL+ & $2,385,685$ & $8,778,753$ & 456,106 & 151,551 & $11,772,095$ & 200,060 \\
\hline MINNESOTA & bGLM+ & $3,006,117$ & $12,871,946$ & 951,151 & 170,594 & $16,999,808$ & 251,211 \\
\hline MISSOURI & GLM+ & 967,024 & $7,248,586$ & 178,571 & 65,080 & $8,459,261$ & 91,395 \\
\hline MONTREAL & GLM+ & $2,641,805$ & $9,427,747$ & 154,871 & 23,028 & $12,247,451$ & 139,899 \\
\hline NEBRASKA & $\mathrm{L}+$ & 585,693 & $5,689,064$ & 163,025 & 21,062 & $6,458,844$ & 102,111 \\
\hline NEW MEXICO & GLM+ & $1,348,879$ & $5,911,858$ & 223,373 & 504,899 & $7,989,009$ & 168,134 \\
\hline NEW YORK & bGLM+ & $5,209,920$ & $10,111,441$ & $2,316,400$ & 280,697 & $17,918,458$ & 509,153 \\
\hline NORTH CAROLINA & GLM+ & $3,030,479$ & $6,810,130$ & $6,266,828$ & 60,488 & $16,167,925$ & 228,338 \\
\hline NORTH CAROLINA STATE & G+ & $1,400,664$ & $7,268,906$ & 89,911 & 916,729 & $9,676,210$ & 83,293 \\
\hline NORTHWESTERN & BGLM+ & $3,607,610$ & $9,014,225$ & 826,278 & 452,569 & $13,900,682$ & 221,801 \\
\hline NOTRE DAME & bGL+ & $4,489,450$ & $7,194,446$ & UA/NA & 52,931 & $11,736,827$ & 83,445 \\
\hline $\mathrm{OHIO}$ & $\mathrm{BbGM}+$ & 919,119 & $4,032,388$ & 331,634 & 181,686 & $5,464,827$ & 36,515 \\
\hline OHIO STATE & bGLM+ & $2,511,656$ & $8,789,376$ & 739,367 & 627,117 & $12,667,516$ & 344,008 \\
\hline OKLAHOMA & GLM+ & $3,326,015$ & $8,702,979$ & $1,859,904$ & 517,410 & $14,406,308$ & 218,987 \\
\hline OKLAHOMA STATE & BGM+ & $\mathrm{UA} / \mathrm{NA}$ & $\mathrm{UA} / \mathrm{NA}$ & $\mathrm{UA} / \mathrm{NA}$ & UA/NA & $6,912,637$ & 32,841 \\
\hline OREGON & BGL+ & $1,159,041$ & $4,085,978$ & $\mathrm{UA} / \mathrm{NA}$ & $1,142,072$ & $6,387,091$ & 66,821 \\
\hline PENNSYLVANIA & GLM+ & $3,797,236$ & $10,215,771$ & 640,097 & 571,533 & $15,224,637$ & 280,195 \\
\hline
\end{tabular}




\section{EXPENDITURES}

Salaries \& Wages Salaries \& Wages Salaries \& Wages Total Salaries Other Operating Total Library Professional Staff Support Staff Student Assistants \&Wages Expenditures Expenditures
(18a)
(18b)
(18c)
(18)
(20)
(21) (Survey Question Number)

\begin{tabular}{|c|c|c|c|c|c|c|}
\hline $3,086,844$ & $2,583,814$ & 626,364 & $6,297,022$ & $3,843,663$ & $20,149,427$ & HOUSTON \\
\hline $2,382,012$ & $1,786,351$ & 535,066 & $4,703,429$ & 553,566 & $9,347,005$ & HOWARD \\
\hline $3,733,258$ & $3,721,575$ & 529,681 & $7,984,514$ & $1,913,357$ & $20,616,384$ & ILLINOIS, CHICAGO \\
\hline $11,839,327$ & $7,783,740$ & $2,237,202$ & $21,860,269$ & $4,943,339$ & $41,600,882$ & ILLINOIS, URBANA \\
\hline $8,583,861$ & $5,000,975$ & $1,921,056$ & $15,505,892$ & $2,693,223$ & $33,147,603$ & INDIANA \\
\hline $6,869,124$ & $5,553,659$ & $1,032,261$ & $13,455,044$ & $1,623,439$ & $29,517,181$ & IOWA \\
\hline $3,223,185$ & $3,765,735$ & 471,233 & $7,460,153$ & $3,123,705$ & $23,250,210$ & IOWA STATE \\
\hline $5,995,234$ & $4,765,101$ & 162,876 & $10,923,211$ & $3,560,865$ & $29,124,364$ & JOHNS HOPKINS \\
\hline $6,018,641$ & $3,758,683$ & $1,067,342$ & $10,844,666$ & $2,458,941$ & $23,042,922$ & KANSAS \\
\hline $3,572,061$ & $1,349,541$ & 408,121 & $5,329,723$ & $2,944,949$ & $12,551,309$ & KENT STATE \\
\hline $5,100,284$ & $2,745,490$ & 681,806 & $8,527,580$ & $2,327,502$ & $21,734,374$ & KENTUCKY \\
\hline $3,676,998$ & $6,141,418$ & 22,424 & $9,840,839$ & 574,607 & $20,099,031$ & LAVAL \\
\hline $3,538,345$ & $2,152,620$ & 530,028 & $6,220,993$ & 841,535 & $13,428,011$ & LOUISIANA STATE \\
\hline $3,138,327$ & $2,743,824$ & 482,486 & $6,364,637$ & $3,560,918$ & $19,533,056$ & LOUISVILLE \\
\hline $4,718,258$ & $5,896,114$ & 401,719 & $11,016,091$ & $4,170,119$ & $28,349,450$ & MCGILL \\
\hline $3,476,542$ & $3,084,958$ & 544,805 & $7,106,305$ & $2,355,226$ & $17,449,235$ & MCMASTER \\
\hline $4,823,435$ & $5,007,673$ & 682,276 & $10,513,384$ & $1,832,792$ & $20,232,942$ & MANITOBA \\
\hline $8,077,604$ & $4,004,964$ & 855,800 & $12,938,368$ & $3,249,588$ & $25,783,433$ & MARYLAND \\
\hline $3,787,468$ & $3,157,324$ & 621,155 & $7,565,947$ & $1,350,309$ & $15,403,001$ & MASSACHUSETTS \\
\hline $8,472,345$ & $3,922,198$ & 393,994 & $12,788,537$ & $3,218,187$ & $25,260,636$ & MIT \\
\hline $5,300,034$ & $3,624,704$ & 747,011 & $9,671,749$ & $3,144,000$ & $26,584,581$ & MIAMI \\
\hline $11,937,143$ & $12,090,548$ & $1,769,852$ & $25,797,543$ & $4,392,321$ & $53,134,323$ & MICHIGAN \\
\hline $5,397,870$ & $5,161,873$ & $1,339,269$ & $11,899,012$ & $2,540,397$ & $26,411,564$ & MICHIGAN STATE \\
\hline $8,189,906$ & $8,980,248$ & $1,408,782$ & $18,578,936$ & $5,395,625$ & $41,225,580$ & MINNESOTA \\
\hline $3,718,963$ & $2,743,607$ & 501,248 & $6,963,818$ & $1,721,278$ & $17,235,752$ & MISSOURI \\
\hline $6,909,157$ & $9,454,722$ & 150,207 & $16,514,087$ & 910,783 & $29,812,219$ & MONTREAL \\
\hline $3,547,791$ & $3,067,232$ & 672,778 & $7,287,801$ & $1,381,865$ & $15,230,621$ & NEBRASKA \\
\hline $4,540,578$ & $5,339,673$ & 582,760 & $10,463,011$ & $2,189,493$ & $20,809,647$ & NEW MEXICO \\
\hline $9,375,985$ & $10,017,180$ & $1,493,054$ & $20,886,219$ & $6,579,356$ & $45,893,186$ & NEW YORK \\
\hline $11,269,423$ & $7,895,360$ & $1,081,379$ & $20,246,162$ & $4,127,565$ & $40,769,990$ & NORTH CAROLINA \\
\hline $7,882,273$ & $3,660,695$ & 634,839 & $12,177,807$ & $6,546,495$ & $28,483,805$ & NORTH CAROLINA STATE \\
\hline $7,631,880$ & $4,863,001$ & 796,199 & $13,291,080$ & $2,857,200$ & $30,270,763$ & NORTHWESTERN \\
\hline $5,478,312$ & $4,721,901$ & 618,159 & $10,818,372$ & $2,360,654$ & $24,999,298$ & NOTRE DAME \\
\hline $2,683,731$ & $2,659,429$ & 700,301 & $6,043,461$ & $1,724,039$ & $13,268,842$ & $\mathrm{OHIO}$ \\
\hline $10,358,308$ & $5,295,005$ & $1,952,290$ & $17,605,603$ & $8,201,238$ & $38,818,365$ & OHIO STATE \\
\hline $3,355,122$ & $2,553,597$ & $1,093,656$ & $7,002,375$ & $2,237,943$ & $23,865,613$ & OKLAHOMA \\
\hline $4,972,021$ & $2,886,768$ & 572,900 & $8,431,689$ & $2,509,742$ & $17,886,909$ & OKLAHOMA STATE \\
\hline $4,157,965$ & $3,029,332$ & $1,106,893$ & $8,294,190$ & $1,326,614$ & $16,074,716$ & OREGON \\
\hline $8,435,843$ & $8,389,773$ & 912,197 & $17,737,813$ & $6,344,431$ & $39,587,076$ & PENNSYLVANIA \\
\hline
\end{tabular}




\section{EXPENDITURES}

Monographs Current Serials Other Library Miscellaneous Total Library Contract Materials Materials Materials Binding

(Survey Question Number)

(16a)

(16b)

(16c)

(16d)

(16)

(17)

\begin{tabular}{|c|c|c|c|c|c|c|c|}
\hline INSTITUTION & Notes & & & & & & \\
\hline PENNSYLVANIA STATE & BbGLM+ & $4,005,270$ & $12,760,610$ & $1,728,337$ & 0 & $18,494,217$ & 347,811 \\
\hline PITTSBURGH & BGLM+ & $2,923,074$ & $9,523,942$ & $1,314,477$ & 444,777 & $14,206,270$ & 145,387 \\
\hline PRINCETON & bG+ & $8,437,800$ & $10,709,460$ & $2,085,367$ & 701,864 & $21,934,491$ & 547,968 \\
\hline PURDUE & G+ & $1,376,389$ & $7,921,059$ & $1,665,301$ & 262,537 & $11,225,286$ & 46,994 \\
\hline QUEEN'S & $\mathrm{LM}+$ & $1,167,682$ & $6,500,290$ & 102,473 & 557,676 & $8,328,121$ & 62,882 \\
\hline RICE & G+ & $3,223,688$ & $6,443,468$ & 317,604 & 476,169 & $10,460,929$ & 49,663 \\
\hline ROCHESTER & $\mathrm{bM}+$ & $1,193,180$ & $6,984,764$ & 31,050 & 57,777 & $8,266,771$ & 90,372 \\
\hline RUTGERS & BGL+ & $1,873,521$ & $8,501,656$ & 753,126 & 351,107 & $11,479,410$ & 85,535 \\
\hline SASKATCHEWAN & GLM+ & $2,146,004$ & $7,007,379$ & UA/NA & 448,666 & $9,602,049$ & 54,238 \\
\hline SOUTH CAROLINA & $\mathrm{LM}+$ & $1,429,841$ & $6,377,527$ & 212,985 & 124,351 & $8,144,704$ & 88,428 \\
\hline SOUTHERN CALIFORNIA & BGLM+ & $3,782,005$ & $8,888,694$ & $4,407,022$ & 276,450 & $17,354,171$ & 139,030 \\
\hline SOUTHERN ILLINOIS & LM+ & 711,745 & $6,012,763$ & 227,706 & 319,358 & $7,271,572$ & 110,447 \\
\hline SUNY-ALBANY & $\mathrm{G}^{+}$ & 549,758 & $4,362,545$ & 19,229 & 267,369 & $5,198,901$ & 40,681 \\
\hline SUNY-BUFFALO & GLM+ & $1,662,906$ & $6,692,869$ & 596,113 & 0 & $8,951,888$ & 42,034 \\
\hline SUNY-STONY BROOK & $\mathrm{bM}+$ & 301,426 & $6,094,482$ & 158,283 & 396,549 & $6,950,740$ & 26,316 \\
\hline SYRACUSE & GL+ & $1,353,362$ & $5,666,523$ & 551,412 & UA/NA & $7,571,297$ & 52,339 \\
\hline TEMPLE & BGLM+ & $2,336,860$ & $6,337,644$ & $3,240,760$ & 27,397 & $11,942,661$ & 117,023 \\
\hline TENNESSEE & BbGLM+ & $2,346,711$ & $8,805,394$ & 554,299 & 440,339 & $12,146,743$ & 61,763 \\
\hline TEXAS & GL+ & $2,627,065$ & $10,204,174$ & $4,127,763$ & 468,072 & $17,427,074$ & 183,555 \\
\hline TEXAS A\&M & $\mathrm{BbG}+$ & $4,801,703$ & $12,305,942$ & 661,045 & 0 & $17,768,690$ & 99,587 \\
\hline TEXAS TECH & BLM+ & $2,320,596$ & $8,238,684$ & 256,325 & 698,808 & $11,514,413$ & 73,677 \\
\hline TORONTO & BLM+ & $7,374,842$ & $10,802,507$ & $5,132,477$ & $\mathrm{UA} / \mathrm{NA}$ & $23,309,826$ & 308,780 \\
\hline TULANE & BGLM+ & $1,960,928$ & $6,463,567$ & 309,200 & 15,620 & $8,749,315$ & 120,287 \\
\hline UTAH & $\mathrm{LM}+$ & $1,826,441$ & $5,349,353$ & 122,336 & 16,728 & $7,314,858$ & 72,903 \\
\hline VANDERBILT & GLM+ & $1,378,478$ & $9,576,572$ & 65,258 & 43,400 & $11,063,708$ & 100,405 \\
\hline VIRGINIA & $\mathrm{LM}+$ & $2,980,240$ & $7,821,461$ & $2,148,945$ & 156,416 & $13,107,062$ & 89,609 \\
\hline VIRGINIA TECH & G+ & $1,204,203$ & $5,335,345$ & UA/NA & 615,565 & $7,155,113$ & 52,672 \\
\hline WASHINGTON & BGLM+ & $3,770,109$ & $12,131,204$ & 421,704 & 334,192 & $16,657,209$ & 207,156 \\
\hline WASHINGTON STATE & $\mathrm{BbG}+$ & 952,370 & $4,985,493$ & 10,729 & 204,287 & $6,152,879$ & 47,244 \\
\hline WASHINGTON U.-ST. LOUIS & GLM+ & $2,135,058$ & $8,619,649$ & $2,017,123$ & 455 & $12,772,285$ & 96,744 \\
\hline WATERLOO & G+ & 747,903 & $4,774,658$ & 82,090 & 320,433 & $5,925,083$ & 50,102 \\
\hline WAYNE STATE & GLM+ & 620,886 & $7,990,111$ & 0 & 90,075 & $8,701,072$ & 66,672 \\
\hline WESTERN ONTARIO & GL+ & $1,936,536$ & $8,248,564$ & 0 & 0 & $10,185,101$ & 111,643 \\
\hline WISCONSIN & GLM+ & $2,111,462$ & $6,351,064$ & $2,847,371$ & 401,091 & $11,710,988$ & 208,481 \\
\hline YALE & GLM+ & $15,345,869$ & $5,670,704$ & $18,089,322$ & 0 & $39,105,895$ & 521,338 \\
\hline YORK & BGL+ & $2,244,864$ & $7,359,970$ & 212,768 & 93,790 & $9,911,392$ & 125,098 \\
\hline BOSTON PUBLIC & bG+ & $3,572,175$ & 939,999 & $1,090,752$ & 36,624 & $5,639,550$ & 13,609 \\
\hline CENTER FOR RESEARCH LIBRARIES & & 94,562 & 299,786 & 852,072 & 25,077 & $1,271,497$ & \\
\hline LIBRARY OF CONGRESS & bG+ & $5,935,396$ & $5,415,352$ & $7,968,855$ & 56,700 & $19,376,303$ & $1,487,727$ \\
\hline
\end{tabular}




\section{EXPENDITURES}

Salaries \& Wages Salaries \& Wages Salaries \& Wages Total Salaries Other Operating Total Library Professional Staff

Support Staff Student Assistants

$(18 b)$ (18c)
\& Wages

(18)

Expenditures Expenditures

(18a)

(20)

(21) (Survey Question Number)

\begin{tabular}{|c|c|c|c|c|c|c|}
\hline $11,013,637$ & $13,713,303$ & 685,795 & $25,412,735$ & $5,196,367$ & $49,451,130$ & PENNSYLVANIA STATE \\
\hline $6,927,317$ & $5,004,991$ & 949,735 & $12,882,043$ & $2,911,601$ & $30,145,301$ & PITTSBURGH \\
\hline $9,881,292$ & $8,740,756$ & $1,031,970$ & $19,654,018$ & $5,500,247$ & $47,636,724$ & PRINCETON \\
\hline $5,746,818$ & $4,190,208$ & 972,615 & $10,909,641$ & $2,648,370$ & $24,830,291$ & PURDUE \\
\hline $3,183,915$ & $3,859,429$ & 287,529 & $7,330,873$ & $1,217,313$ & $16,939,189$ & QUEEN'S \\
\hline $3,565,896$ & $1,215,856$ & 176,233 & $4,957,985$ & 778,187 & $16,246,764$ & RICE \\
\hline $5,709,277$ & $1,800,905$ & 500,164 & $8,010,346$ & $2,520,104$ & $18,887,593$ & ROCHESTER \\
\hline $7,325,145$ & $11,096,162$ & $1,182,127$ & $19,603,434$ & $2,050,656$ & $33,219,035$ & RUTGERS \\
\hline $3,721,991$ & $3,006,224$ & 309,971 & $7,038,186$ & $1,540,740$ & $18,235,214$ & SASKATCHEWAN \\
\hline $3,801,759$ & $3,182,863$ & 646,431 & $7,631,053$ & $3,420,807$ & $19,284,992$ & SOUTH CAROLINA \\
\hline $9,719,360$ & $5,866,634$ & $1,549,120$ & $17,135,114$ & $4,364,993$ & $38,993,308$ & SOUTHERN CALIFORNIA \\
\hline $2,865,488$ & $2,859,960$ & 856,744 & $6,582,192$ & $1,264,100$ & $15,228,311$ & SOUTHERN ILLINOIS \\
\hline $3,973,614$ & $2,267,806$ & 483,684 & $6,725,104$ & 541,643 & $12,506,329$ & SUNY-ALBANY \\
\hline $7,255,032$ & $2,409,384$ & 797,820 & $10,462,236$ & $1,570,492$ & $21,026,650$ & SUNY-BUFFALO \\
\hline $4,690,421$ & 972,226 & 459,298 & $6,121,945$ & 673,702 & $13,772,703$ & SUNY-STONY BROOK \\
\hline $4,375,375$ & $3,972,839$ & 472,306 & $8,820,520$ & $1,896,018$ & $18,340,174$ & SYRACUSE \\
\hline $5,230,804$ & $2,380,956$ & 730,039 & $8,341,799$ & $2,459,950$ & $22,861,433$ & TEMPLE \\
\hline $5,481,220$ & $3,672,416$ & 469,807 & $9,623,443$ & $1,683,631$ & $23,515,580$ & TENNESSEE \\
\hline $9,141,083$ & $11,629,525$ & $1,648,745$ & $22,419,353$ & $6,175,281$ & $46,205,263$ & TEXAS \\
\hline $8,549,863$ & $3,871,353$ & $1,101,848$ & $13,523,064$ & $4,983,210$ & $36,374,551$ & TEXAS A\&M \\
\hline $5,421,156$ & $3,660,892$ & $1,301,170$ & $10,383,218$ & $4,078,135$ & $26,049,443$ & TEXAS TECH \\
\hline $14,067,776$ & $13,931,041$ & $4,015,851$ & $32,014,667$ & $4,972,166$ & $60,605,439$ & TORONTO \\
\hline $3,184,409$ & $1,886,413$ & 298,055 & $5,368,877$ & 637,428 & $14,875,907$ & TULANE \\
\hline $4,573,610$ & $5,834,023$ & $1,370,436$ & $11,778,069$ & $4,782,547$ & $23,948,377$ & UTAH \\
\hline $6,554,062$ & $3,186,842$ & 498,659 & $10,239,563$ & $2,991,816$ & $24,395,492$ & VANDERBILT \\
\hline $7,059,098$ & $9,073,465$ & 779,230 & $16,911,793$ & $4,139,609$ & $34,248,073$ & VIRGINIA \\
\hline $2,447,058$ & $2,978,713$ & 398,853 & $5,824,624$ & $1,144,668$ & $14,177,077$ & VIRGINIA TECH \\
\hline $12,306,731$ & $7,907,735$ & $2,510,484$ & $22,724,950$ & $4,366,816$ & $43,956,131$ & WASHINGTON \\
\hline $3,100,145$ & $3,135,530$ & 621,978 & $6,857,653$ & $1,299,060$ & $14,356,836$ & WASHINGTON STATE \\
\hline $6,180,179$ & $3,579,945$ & 693,202 & $10,453,326$ & $4,908,148$ & $28,230,503$ & WASHINGTON U.-ST. LOUIS \\
\hline $2,337,313$ & $3,223,925$ & 608,810 & $6,170,048$ & $1,275,001$ & $13,420,234$ & WATERLOO \\
\hline $5,389,243$ & $2,967,920$ & $1,040,925$ & $9,398,088$ & $2,924,935$ & $21,090,767$ & WAYNE STATE \\
\hline $3,861,480$ & $3,548,518$ & 284,514 & $7,694,513$ & 641,522 & $18,632,779$ & WESTERN ONTARIO \\
\hline $14,917,256$ & $5,829,800$ & $2,757,000$ & $23,504,056$ & $8,309,231$ & $43,732,756$ & WISCONSIN \\
\hline $21,128,778$ & $15,486,393$ & $1,348,229$ & $37,963,400$ & $11,352,128$ & $88,942,761$ & YALE \\
\hline $5,152,537$ & $4,584,491$ & 938,427 & $10,675,456$ & $2,910,774$ & $23,622,720$ & YORK \\
\hline $12,020,194$ & $12,269,059$ & 597,277 & $24,886,530$ & $14,073,025$ & $44,612,714$ & BOSTON PUBLIC \\
\hline $1,904,815$ & 454,792 & 232,918 & $2,592,525$ & 956,324 & $4,820,346$ & CENTER FOR RESEARCH LIBRARIES \\
\hline $391,815,971$ & . & . & $391,815,971$ & $234,081,000$ & $646,761,001$ & LIBRARY OF CONGRESS \\
\hline
\end{tabular}




\section{EXPENDITURES}

Monographs Current Serials

Other Library Miscellaneous Materials Materials
Total Library Materials

(16d)

(16)
Contract

Binding

(17)

INSTITUTION

(Survey Question Number)

(16a)

(16b)

(16c)

\begin{tabular}{|c|c|c|c|c|c|c|c|}
\hline NATIONAL AGRICULTURAL LIBRARY & G+ & 356,019 & $2,784,103$ & 985,627 & 0 & $4,125,750$ & 0 \\
\hline LIBRARY \& ARCHIVES CANADA & $\mathrm{G}+$ & 473,341 & 536,569 & 113,761 & 176,701 & $1,300,372$ & $\mathrm{UA} / \mathrm{NA}$ \\
\hline NATIONAL LIBRARY OF MEDICINE & $\mathrm{G}+$ & $1,159,589$ & $8,057,753$ & 115,817 & 88,258 & $9,421,417$ & 154,519 \\
\hline NEW YORK PUBLIC & + & $6,075,630$ & $3,535,577$ & $3,047,605$ & $\mathrm{UA} / \mathrm{NA}$ & $12,658,812$ & 655,477 \\
\hline NEW YORK STATE & G+ & 916,261 & $2,520,222$ & $\mathrm{UA} / \mathrm{NA}$ & 0 & $3,436,483$ & 9,600 \\
\hline SMITHSONIAN & $\mathrm{G}+$ & 304,086 & $1,187,847$ & 0 & 51,588 & $1,543,521$ & 1,392 \\
\hline
\end{tabular}

+ - See Footnotes

L - Includes Law library

M- Includes Medical library
G - Government documents not included in serials count B - Includes branch campuses

$\mathrm{b}$ - Basis of volume count is bibliographic
U/A - Unavailable

N/A - Not Applicable

- - no data supplied

\section{Summary Data}

Monographs Current Serials

(Survey Question Number)

(16a)

(16b)

Other Library Miscellaneous Materials Materials

(16c)

\section{University Libraries}

Mean
Median
High
Low
Totals
Number of Libraries Reporting

\section{Nonuniversity Libraries}

Median

Totals

Number of Libraries Reporting

$\begin{array}{rr}2,574,673 & 7,577,613 \\ 2,056,586 & 7,192,136 \\ 15,345,869 & 15,015,672 \\ 244,960 & 3,445,792 \\ 290,938,016 & 856,270,302 \\ 113 & 113\end{array}$

113

113

$$
\begin{array}{r}
1,161,527 \\
331,634 \\
18,089,322 \\
0 \\
124,283,336 \\
107
\end{array}
$$

918,850

$14,174,489$

8

$138,457,826$

$$
\begin{array}{r}
2,520,222 \\
25,277,208
\end{array}
$$

9

$309,825,075$
$881,547,511$
Total Library Materials

(16d)

(16)

Contract Binding

(17)

\section{GRAND TOTALS}

403,987
295,696
$2,877,304$
0
$43,630,606$
108

$11,596,797$

$10,274,939$

$39,105,895$

$4,073,010$

$1,322,034,898$

114

150,125

101,952

$1,061,257$

6,200

$17,114,243$

114 


\section{EXPENDITURES}

Salaries \& Wages Salaries \& Wages Salaries \& Wages Total Salaries Other Operating Total Library Professional Staff Support Staff Student Assistants

\& Wages

Expenditures Expenditures
(18a)
(18b)
(18c)
(18)
(20)
(21) (Survey Question Number)

INSTITUTION

\begin{tabular}{rrrrrrr}
\hline $12,415,165$ & $2,741,081$ & 8,524 & $15,164,770$ & $8,688,707$ & $27,979,227$ & NATIONAL AGRICULTURAL LIBRARY \\
$52,239,961$ & $23,050,585$ & 645,300 & $75,935,847$ & & $77,236,219$ & LIBRARY \& ARCHIVES CANADA \\
$23,936,681$ & $4,389,426$ & 508,994 & $28,835,101$ & $23,863,648$ & $62,274,685$ & NATIONAL LIBRARY OF MEDICINE \\
$20,315,408$ & $9,353,150$ & $1,829,963$ & $31,498,521$ & $9,491,840$ & $54,304,650$ & NEW YORK PUBLIC \\
UA/NA & UA/NA & UA/NA & $6,990,000$ & 874,607 & $11,310,690$ & NEW YORK STATE \\
$5,206,996$ & $2,298,467$ & 14,267 & $7,519,730$ & $1,703,990$ & $10,768,633$ & SMITHSONIAN
\end{tabular}

Figures in italics indicate the inclusion of fringe benefits.

\section{Summary Data}

Salaries \& Wages Salaries \& Wages Salaries \& Wages Total Salaries Other Operating Total Library Professional Staff Support Staff Student Assistants \&Wages Expenditures Expenditures

(18a) (18b)

$(18 c)$

(18)

$(20)$

(21) (Survey Question Number)

$\begin{array}{rr}6,624,980 & 5,117,833 \\ 5,344,639 & 4,003,369 \\ 42,980,437 & 20,781,546 \\ 2,337,313 & 972,226 \\ 755,247,753 & 583,432,988 \\ 114 & 114\end{array}$

$\begin{array}{rr}913,858 & 12,656,672 \\ 689,499 & 10,457,781 \\ 4,015,851 & 65,459,316 \\ 22,424 & 4,703,429 \\ 104,179,831 & 1,442,860,573 \\ 114 & 114\end{array}$

$\begin{array}{rr}3,176,306 & 27,579,900 \\ 2,514,923 & 23,569,150 \\ 21,200,914 & 122,980,135 \\ 495,777 & 9,347,005\end{array}$

$362,098,8663,144,108,579$

114

114

Number of Libraries Reporting

$\begin{array}{rr}16,365,287 & 4,389,426 \\ 519,855,191 & 54,556,560 \\ 8 & 7\end{array}$

$\begin{array}{rr}508,994 & 24,886,530 \\ 3,837,243 & 585,238,995 \\ 7 & 9\end{array}$

$9,090,273$

$293,733,141$

$44,612,714$

8

$940,068,165$

9

Number of Libraries Reporting

Median

Totals

GRAND TOTALS

Nonuniversity Libraries

Median

High

Low

Totals

University Libraries Mean 


\section{Electronic Resources Expenditures}

$\begin{array}{rrr}\text { One-time } & \text { Ongoing } & \text { Total } \\ \text { Electronic Resource } & \text { Electronic Resource Electronic Resource }\end{array}$ Purchases Purchases Purchases
Total

rary Materials

$(22+23)$
(23)

Expenditures Library Materials Budget

(Survey Question Number)

(22)

Notes

INSTITUTION

\begin{tabular}{|c|c|c|c|c|c|c|}
\hline ALABAMA & bGLM+ & 665,707 & $5,070,190$ & $5,735,897$ & $8,207,066$ & 69.89 \\
\hline ALBERTA & GLM+ & $6,035,063$ & $8,279,012$ & $14,314,075$ & $16,761,357$ & 85.40 \\
\hline ARIZONA & bGLM+ & 832,150 & $7,864,124$ & $8,696,274$ & $12,890,572$ & 67.46 \\
\hline ARIZONA STATE & BGL+ & $1,040,877$ & $7,314,788$ & $8,355,665$ & $11,204,849$ & 74.57 \\
\hline AUBURN & + & 1,725 & $4,849,862$ & $4,851,587$ & $5,760,251$ & 84.23 \\
\hline BOSTON & $\mathrm{LM}+$ & 197,587 & $4,761,308$ & $4,958,895$ & $9,375,336$ & 52.89 \\
\hline BOSTON COLLEGE & $\mathrm{L}+$ & 363,765 & $4,976,305$ & $5,340,070$ & $9,161,518$ & 58.29 \\
\hline BRIGHAM YOUNG & GL+ & $\mathrm{UA} / \mathrm{NA}$ & $5,223,576$ & $5,223,576$ & $9,542,790$ & 54.74 \\
\hline BRITISH COLUMBIA & $\mathrm{LM}+$ & 208,999 & $9,012,422$ & $9,221,421$ & $15,366,604$ & 60.01 \\
\hline BROWN & bGM+ & 141,867 & $4,822,055$ & $4,963,922$ & $8,817,845$ & 56.29 \\
\hline CALGARY & GLM+ & 790,253 & $5,932,951$ & $6,723,204$ & $11,788,750$ & 57.03 \\
\hline CALIFORNIA, BERKELEY & $\mathrm{L}+$ & 266,710 & $6,073,193$ & $6,339,903$ & $17,849,390$ & 35.52 \\
\hline CALIFORNIA, DAVIS & GLM+ & 66,500 & $4,822,120$ & $4,888,620$ & $8,717,848$ & 56.08 \\
\hline CALIFORNIA, IRVINE & bGM+ & 554,172 & $4,835,748$ & $5,389,920$ & $9,489,506$ & 56.80 \\
\hline CALIFORNIA, LOS ANGELES & bGLM+ & 347,415 & $4,803,251$ & $5,150,666$ & $14,459,035$ & 35.62 \\
\hline CALIFORNIA, RIVERSIDE & G+ & 33,831 & $2,190,445$ & $2,224,276$ & $4,802,770$ & 46.31 \\
\hline CALIFORNIA, SAN DIEGO & bGM+ & 515,778 & $5,257,095$ & $5,772,873$ & $9,876,233$ & 58.45 \\
\hline CALIFORNIA, SANTA BARBARA & G+ & 46,818 & $3,262,863$ & $3,309,681$ & $5,449,640$ & 60.73 \\
\hline CASE WESTERN RESERVE & GLM+ & 48,822 & $3,770,121$ & $3,818,943$ & $6,707,114$ & 56.94 \\
\hline CHICAGO & bGLM+ & 352,806 & $6,677,677$ & $7,030,483$ & $18,073,119$ & 38.90 \\
\hline CINCINNATI & GLM+ & 443,579 & $5,110,937$ & $5,554,516$ & $10,179,240$ & 54.57 \\
\hline COLORADO & bG+ & 593,956 & $6,228,185$ & $6,822,141$ & $11,394,102$ & 59.87 \\
\hline COLORADO STATE & G+ & 310,780 & $4,431,194$ & $4,741,974$ & $6,509,389$ & 72.85 \\
\hline COLUMBIA & GLM+ & $1,358,703$ & $10,568,113$ & $11,926,816$ & $24,555,907$ & 48.57 \\
\hline CONNECTICUT & BbGLM+ & 567,689 & $6,606,695$ & $7,174,384$ & $10,158,424$ & 70.62 \\
\hline CORNELL & BGL+ & 110,173 & $7,495,686$ & $7,605,859$ & $16,161,049$ & 47.06 \\
\hline DARTMOUTH & bGM+ & 417,730 & $5,871,285$ & $6,289,015$ & $9,399,296$ & 66.91 \\
\hline DELAWARE & G+ & 403,430 & $5,559,063$ & $5,962,493$ & $8,969,172$ & 66.48 \\
\hline DUKE & $\mathrm{LM}+$ & 744,097 & $7,640,753$ & $8,384,850$ & $16,451,465$ & 50.97 \\
\hline EMORY & $\mathrm{BLM}+$ & 976,638 & $5,140,483$ & $6,117,121$ & $16,977,757$ & 36.03 \\
\hline FLORIDA & BbLM+ & 257,861 & $4,240,202$ & $4,498,063$ & $12,261,949$ & 36.68 \\
\hline FLORIDA STATE & BbLM+ & 65,071 & $5,743,510$ & $5,808,581$ & $8,062,835$ & 72.04 \\
\hline GEORGE WASHINGTON & BGLM+ & 165,392 & $5,050,666$ & $5,216,058$ & $11,175,276$ & 46.67 \\
\hline GEORGETOWN & bGLM+ & 295,677 & $4,707,463$ & $5,003,140$ & $12,010,333$ & 41.66 \\
\hline GEORGIA & GL+ & 9,642 & $5,844,505$ & $5,854,147$ & $10,364,778$ & 56.48 \\
\hline GEORGIA TECH & G+ & 38,395 & $4,596,261$ & $4,634,656$ & $6,037,151$ & 76.77 \\
\hline GUELPH & BG+ & 364,976 & $3,481,324$ & $3,846,301$ & $5,818,580$ & 66.10 \\
\hline HARVARD & GLM+ & $10,204,304$ & $\mathrm{UA} / \mathrm{NA}$ & $10,204,304$ & $35,296,483$ & 28.91 \\
\hline HAWAII & GLM+ & 179,945 & $5,456,211$ & $5,636,156$ & $8,105,607$ & 69.53 \\
\hline
\end{tabular}




\section{Electronic Resources ExPENDITURes}

Bibl. Utilities, Networks, etc. Bibl. Utilities, Networks, etc. Library Expenditures External Expenditures

(24a)

(24b)

Computer Hardware and

Software Expenditures

(25)
Document Delivery/

Interlibrary Loan

Expenditures

(26) (Survey Question Number)

INSTITUTION

\begin{tabular}{|c|c|c|c|c|}
\hline 296,770 & 47,500 & 800,185 & 34,690 & ALABAMA \\
\hline $\mathrm{UA} / \mathrm{NA}$ & $\mathrm{UA} / \mathrm{NA}$ & $\mathrm{UA} / \mathrm{NA}$ & UA/NA & ALBERTA \\
\hline 0 & 924,391 & $2,721,362$ & 160,219 & ARIZONA \\
\hline 288,306 & 0 & 220,004 & 192,595 & ARIZONA STATE \\
\hline 181,260 & 166,500 & 681,614 & 91,526 & AUBURN \\
\hline 196,868 & 0 & 287,701 & 93,892 & BOSTON \\
\hline 136,019 & UA/NA & 177,621 & 26,011 & BOSTON COLLEGE \\
\hline 439,099 & UA/NA & 524,466 & 53,015 & BRIGHAM YOUNG \\
\hline 843,005 & 625,584 & 984,237 & 99,262 & BRITISH COLUMBIA \\
\hline 0 & 8,500 & 218,430 & 199,713 & BROWN \\
\hline 244,185 & 263,878 & 343,927 & 104,708 & CALGARY \\
\hline 58,368 & 968,946 & 608,540 & 900,893 & CALIFORNIA, BERKELEY \\
\hline 242,843 & 836,782 & 292,005 & 169,089 & CALIFORNIA, DAVIS \\
\hline 138,513 & 738,672 & 572,410 & 67,227 & CALIFORNIA, IRVINE \\
\hline 498,105 & $1,046,251$ & $1,798,522$ & 314,290 & CALIFORNIA, LOS ANGELES \\
\hline 162,947 & 494,903 & 21,020 & 57,416 & CALIFORNIA, RIVERSIDE \\
\hline $\mathrm{UA} / \mathrm{NA}$ & 753,344 & $1,227,770$ & 127,340 & CALIFORNIA, SAN DIEGO \\
\hline 157,654 & 598,624 & 321,931 & 174,318 & CALIFORNIA, SANTA BARBARA \\
\hline 81,137 & 146,094 & 216,940 & 48,610 & CASE WESTERN RESERVE \\
\hline 514,823 & 250,000 & 503,461 & 227,372 & CHICAGO \\
\hline 153,169 & 528,361 & 639,469 & 8,728 & CINCINNATI \\
\hline 0 & 34,654 & 70,524 & 145,649 & COLORADO \\
\hline 269,671 & 53,700 & 360,659 & 301,411 & COLORADO STATE \\
\hline 429,750 & 0 & 886,567 & 1,636 & COLUMBIA \\
\hline 144,486 & $1,961,515$ & 431,877 & 192,249 & CONNECTICUT \\
\hline 271,480 & 0 & $1,803,780$ & 169,204 & CORNELL \\
\hline UA/NA & $\mathrm{UA} / \mathrm{NA}$ & 248,723 & 126,140 & DARTMOUTH \\
\hline 155,779 & 0 & 229,762 & 91,122 & DELAWARE \\
\hline 373,851 & 1,011 & 411,077 & 400,093 & DUKE \\
\hline 276,166 & 266,367 & 450,653 & 64,154 & EMORY \\
\hline 196,669 & 592,580 & 427,382 & 71,704 & FLORIDA \\
\hline 278,010 & 488,426 & 182,377 & 72,686 & FLORIDA STATE \\
\hline 138,564 & 0 & 403,291 & 102,405 & GEORGE WASHINGTON \\
\hline 324,988 & 5,298 & 438,814 & 53,818 & GEORGETOWN \\
\hline 13,396 & 0 & 52,426 & 603 & GEORGIA \\
\hline 28,000 & 0 & 299,837 & 325,168 & GEORGIA TECH \\
\hline 176,798 & 243,465 & 402,184 & 78,347 & GUELPH \\
\hline $2,367,622$ & $\mathrm{UA} / \mathrm{NA}$ & $1,692,445$ & UA/NA & HARVARD \\
\hline 200,173 & 421 & 408,110 & 170,684 & HAWAII \\
\hline
\end{tabular}




\section{Electronic Resources Expenditures}

$\begin{array}{rrr}\text { One-time } & \text { Ongoing } & \text { Total } \\ \text { Electronic Resource } & \text { Electronic Resource Electronic Resource }\end{array}$ Purchases Purchases Purchases

Total

Electronic Resources Library Materials as a $\%$ of Expenditures Library Materials Budget

(Survey Question Number)

(22)

(23)

$(22+23)$

(16)

INSTITUTION

Notes

\begin{tabular}{|c|c|c|c|c|c|c|}
\hline HOUSTON & $\mathrm{bL}+$ & $1,093,789$ & $4,776,358$ & $5,870,147$ & $9,903,271$ & 59.27 \\
\hline HOWARD & bLM+ & 23,000 & $2,524,396$ & $2,547,396$ & $4,073,010$ & 62.54 \\
\hline ILLINOIS, CHICAGO & $\mathrm{M}+$ & 22,003 & $1,508,822$ & $1,530,825$ & $10,686,348$ & 14.33 \\
\hline ILLINOIS, URBANA & bGL+ & 568,194 & $7,288,937$ & $7,857,131$ & $14,529,554$ & 54.08 \\
\hline INDIANA & $\mathrm{L}+$ & $1,006,825$ & $6,554,374$ & $7,561,199$ & $14,794,891$ & 51.11 \\
\hline IOWA & $\mathrm{LM}+$ & 381,763 & $7,284,210$ & $7,665,973$ & $14,278,353$ & 53.69 \\
\hline IOWA STATE & G+ & 75,567 & $6,421,069$ & $6,496,636$ & $12,560,760$ & 51.72 \\
\hline JOHNS HOPKINS & $\mathrm{BbGM}+$ & 886,462 & $10,257,317$ & $11,143,779$ & $14,572,377$ & 76.47 \\
\hline KANSAS & BbGLM+ & 623,172 & $5,350,371$ & $5,973,543$ & $9,621,889$ & 62.08 \\
\hline KENT STATE & $\mathrm{BbG}+$ & 115,265 & $2,028,199$ & $2,143,464$ & $4,203,944$ & 50.99 \\
\hline KENTUCKY & GLM+ & 540,397 & $5,744,946$ & $6,285,343$ & $10,769,278$ & 58.36 \\
\hline LAVAL & GLM+ & 160,794 & $4,377,192$ & $4,537,986$ & $9,632,798$ & 47.11 \\
\hline LOUISIANA STATE & GLM+ & 5,891 & $4,094,066$ & $4,099,957$ & $6,323,242$ & 64.84 \\
\hline LOUISVILLE & $\mathrm{BGM}+$ & 75,153 & $4,025,678$ & $4,100,831$ & $9,487,675$ & 43.22 \\
\hline MCGILL & $\mathrm{BbGLM+}$ & 637,046 & $7,030,070$ & $7,667,117$ & $12,989,455$ & 59.03 \\
\hline MCMASTER & bGM+ & 162,830 & $3,939,890$ & $4,102,720$ & $7,981,505$ & 51.40 \\
\hline MANITOBA & GLM+ & 554,844 & $3,657,671$ & $4,212,515$ & $7,756,008$ & 54.31 \\
\hline MARYLAND & G+ & 216,849 & $6,058,271$ & $6,275,120$ & $9,499,472$ & 66.06 \\
\hline MASSACHUSETTS & G+ & 109,922 & $4,262,715$ & $4,372,637$ & $6,417,445$ & 68.14 \\
\hline MIT & G+ & 313,435 & $5,450,883$ & $5,764,318$ & $9,051,913$ & 63.68 \\
\hline MIAMI & bGLM+ & 583,936 & $8,807,744$ & $9,391,680$ & $13,694,535$ & 68.58 \\
\hline MICHIGAN & GLM+ & $1,341,003$ & $9,096,818$ & $10,437,821$ & $22,565,018$ & 46.26 \\
\hline MICHIGAN STATE & GL+ & 833,349 & $6,323,386$ & $7,156,735$ & $11,772,095$ & 60.79 \\
\hline MINNESOTA & bGLM+ & 683,028 & $8,644,910$ & $9,327,938$ & $16,999,808$ & 54.87 \\
\hline MISSOURI & GLM+ & 35,981 & $6,421,787$ & $6,457,768$ & $8,459,261$ & 76.34 \\
\hline MONTREAL & GLM+ & 210,409 & $7,021,687$ & $7,232,096$ & $12,247,451$ & 59.05 \\
\hline NEBRASKA & $\mathrm{L}+$ & 185,643 & $4,166,172$ & $4,351,815$ & $6,458,844$ & 67.38 \\
\hline NEW MEXICO & GLM+ & 352,492 & $3,851,215$ & $4,203,707$ & $7,989,009$ & 52.62 \\
\hline NEW YORK & bGLM+ & $1,314,407$ & $8,559,647$ & $9,874,054$ & $17,918,458$ & 55.11 \\
\hline NORTH CAROLINA & GLM+ & $1,169,773$ & $7,395,753$ & $8,565,526$ & $16,167,925$ & 52.98 \\
\hline NORTH CAROLINA STATE & G+ & 238,670 & $4,061,186$ & $4,299,856$ & $9,676,210$ & 44.44 \\
\hline NORTHWESTERN & BGLM+ & 705,000 & $7,133,852$ & $7,838,852$ & $13,900,682$ & 56.39 \\
\hline NOTRE DAME & bGL+ & 602,975 & $5,111,341$ & $5,714,316$ & $11,736,827$ & 48.69 \\
\hline $\mathrm{OHIO}$ & $\mathrm{BbGM+}$ & 338,370 & $2,687,247$ & $3,025,617$ & $5,464,827$ & 55.37 \\
\hline OHIO STATE & bGLM+ & 274,493 & $5,324,103$ & $5,598,596$ & $12,667,516$ & 44.20 \\
\hline OKLAHOMA & GLM+ & $1,799,746$ & $3,266,706$ & $5,066,452$ & $14,406,308$ & 35.17 \\
\hline OKLAHOMA STATE & $\mathrm{BGM}+$ & UA/NA & UA/NA & & $6,912,637$ & . \\
\hline OREGON & BGL+ & 126,662 & $2,975,304$ & $3,101,966$ & $6,387,091$ & 48.57 \\
\hline PENNSYLVANIA & GLM+ & 628,201 & $6,870,355$ & $7,498,556$ & $15,224,637$ & 49.25 \\
\hline
\end{tabular}




\section{Electronic Resources ExPENDITURes}

Bibl. Utilities, Networks, etc. Bibl. Utilities, Networks, etc. Library Expenditures

External Expenditure

Computer Hardware and

Software Expenditures

(24a)

(24b)

(25)
Document Delivery/

Interlibrary Loan

Expenditures

(26) (Survey Question Number)

INSTITUTION

\begin{tabular}{|c|c|c|c|c|}
\hline 602,002 & 0 & 990,220 & 21,340 & HOUSTON \\
\hline \multirow{2}{*}{$\begin{array}{r}113,103 \\
.\end{array}$} & \multirow[t]{2}{*}{4,500} & 68,987 & 24,410 & HOWARD \\
\hline & & 36,799 & 32,594 & ILLINOIS, CHICAGO \\
\hline 557,362 & UA/NA & 513,985 & 4,727 & ILLINOIS, URBANA \\
\hline 182,682 & 229,540 & 371,017 & 307,448 & INDIANA \\
\hline 186,995 & 0 & 313,832 & 189,738 & IOWA \\
\hline 100,084 & 0 & 533,036 & 163,781 & IOWA STATE \\
\hline 28,503 & 0 & 400,460 & 42,036 & JOHNS HOPKINS \\
\hline 320,375 & 5,750 & 419,709 & 177,872 & KANSAS \\
\hline 68,848 & 595,125 & 428,474 & 28,667 & KENT STATE \\
\hline 212,280 & 14,065 & 509,333 & 249,035 & KENTUCKY \\
\hline UA/NA & UA/NA & UA/NA & 48,203 & LAVAL \\
\hline 343,161 & 107,849 & 25,555 & 76,839 & LOUISIANA STATE \\
\hline $\mathrm{UA} / \mathrm{NA}$ & 143,149 & 568,895 & 53,802 & LOUISVILLE \\
\hline . & . & 998,059 & 42,371 & MCGILL \\
\hline 0 & 0 & 552,438 & 42,505 & MCMASTER \\
\hline 0 & 0 & 408,768 & 145,229 & MANITOBA \\
\hline 165,298 & 318,825 & 35,666 & 333,056 & MARYLAND \\
\hline 247,449 & 75,000 & 217,021 & 106,340 & MASSACHUSETTS \\
\hline 104,699 & 0 & 538,162 & 118,616 & MIT \\
\hline 211,106 & 0 & $1,072,278$ & 85,035 & MIAMI \\
\hline 412,059 & 3,500 & $2,744,741$ & 266,240 & MICHIGAN \\
\hline 376,743 & UA/NA & 471,617 & 267,045 & MICHIGAN STATE \\
\hline 242,899 & 438,130 & $1,727,310$ & 256,471 & MINNESOTA \\
\hline 145,211 & 0 & 169,743 & 49,849 & MISSOURI \\
\hline 70,153 & 0 & 412,333 & 202,806 & MONTREAL \\
\hline 46,007 & 4,817 & 523,574 & 54,897 & NEBRASKA \\
\hline 550,296 & 15,194 & 486,532 & 55,546 & NEW MEXICO \\
\hline 76,906 & $\mathrm{UA} / \mathrm{NA}$ & $1,231,069$ & 286,215 & NEW YORK \\
\hline 167,755 & $\mathrm{UA} / \mathrm{NA}$ & $1,014,805$ & 172,857 & NORTH CAROLINA \\
\hline 217,274 & & 694,707 & 203,201 & NORTH CAROLINA STATE \\
\hline 152,244 & UA/NA & 954,048 & 168,864 & NORTHWESTERN \\
\hline 84,431 & 199,626 & 956,217 & 108,022 & NOTRE DAME \\
\hline 128,993 & 515,568 & 406,924 & 60,921 & $\mathrm{OHIO}$ \\
\hline 167,524 & $1,234,901$ & 472,267 & 95,482 & OHIO STATE \\
\hline 314,379 & 45,987 & 856,866 & 108,875 & OKLAHOMA \\
\hline UA/NA & UA/NA & UA/NA & 97,604 & OKLAHOMA STATE \\
\hline 281,877 & 43,502 & 552,181 & 97,382 & OREGON \\
\hline 0 & 739,549 & 986,394 & 520,391 & PENNSYLVANIA \\
\hline
\end{tabular}




\section{Electronic Resources Expenditures}

$\begin{array}{rrr}\text { One-time } & \text { Ongoing } & \text { Total } \\ \text { Electronic Resource } & \text { Electronic Resource Electronic Resource }\end{array}$ Purchases
Purchases
Total
Library Materials Purchases
$(22+23)$
Expenditure

(16)
(23)

\begin{tabular}{|c|c|c|c|c|c|c|}
\hline INSTITUTION & Notes & & & & & \\
\hline PENNSYLVANIA STATE & BbGLM+ & 859,054 & $10,300,937$ & $11,159,991$ & $18,494,217$ & 60.34 \\
\hline PITTSBURGH & BGLM+ & 363,727 & $8,972,527$ & $9,336,254$ & $14,206,270$ & 65.72 \\
\hline PRINCETON & bG+ & 859,407 & $7,929,825$ & $8,789,232$ & $21,934,491$ & 40.07 \\
\hline PURDUE & $\mathrm{G}^{+}$ & 145,216 & $7,200,363$ & $7,345,579$ & $11,225,286$ & 65.44 \\
\hline QUEEN'S & $\mathrm{LM}+$ & UA/NA & $4,960,207$ & $4,960,207$ & $8,328,121$ & 59.56 \\
\hline RICE & G+ & 342,598 & $4,091,978$ & $4,434,576$ & $10,460,929$ & 42.39 \\
\hline ROCHESTER & $\mathrm{bM}+$ & 112,967 & $3,628,536$ & $3,741,503$ & $8,266,771$ & 45.26 \\
\hline RUTGERS & BGL+ & 931,129 & $6,129,451$ & $7,060,580$ & $11,479,410$ & 61.51 \\
\hline SASKATCHEWAN & GLM+ & 377,354 & $3,943,672$ & $4,321,026$ & $9,602,049$ & 45.00 \\
\hline SOUTH CAROLINA & $\mathrm{LM}+$ & 125,794 & $4,736,318$ & $4,862,112$ & $8,144,704$ & 59.70 \\
\hline SOUTHERN CALIFORNIA & BGLM+ & $1,460,510$ & $5,216,003$ & $6,676,513$ & $17,354,171$ & 38.47 \\
\hline SOUTHERN ILLINOIS & $\mathrm{LM}+$ & 141,550 & $4,120,239$ & $4,261,789$ & $7,271,572$ & 58.61 \\
\hline SUNY-ALBANY & $\mathrm{G}^{+}$ & 5,942 & $1,912,961$ & $1,918,903$ & $5,198,901$ & 36.91 \\
\hline SUNY-BUFFALO & GLM+ & 658,500 & $5,393,141$ & $6,051,641$ & $8,951,888$ & 67.60 \\
\hline SUNY-STONY BROOK & $\mathrm{bM}+$ & 296,145 & $4,179,677$ & $4,475,822$ & $6,950,740$ & 64.39 \\
\hline SYRACUSE & GL+ & 349,243 & $4,160,393$ & $4,509,636$ & $7,571,297$ & 59.56 \\
\hline TEMPLE & BGLM+ & $1,102,351$ & $6,698,086$ & $7,800,437$ & $11,942,661$ & 65.32 \\
\hline TENNESSEE & BbGLM+ & 748,782 & $6,927,256$ & $7,676,038$ & $12,146,743$ & 63.19 \\
\hline TEXAS & GL+ & $1,863,974$ & $6,474,689$ & $8,338,663$ & $17,427,074$ & 47.85 \\
\hline TEXAS A\&M & $\mathrm{BbG}+$ & 747,789 & $9,796,026$ & $10,543,815$ & $17,768,690$ & 59.34 \\
\hline TEXAS TECH & BLM+ & 562,844 & $5,291,177$ & $5,854,021$ & $11,514,413$ & 50.84 \\
\hline TORONTO & BLM+ & $1,230,153$ & $10,215,295$ & $11,445,449$ & $23,309,826$ & 49.10 \\
\hline TULANE & BGLM+ & $1,389,181$ & $4,259,138$ & $5,648,319$ & $8,749,315$ & 64.56 \\
\hline UTAH & $\mathrm{LM}+$ & UA/NA & $4,500,465$ & $4,500,465$ & $7,314,858$ & 61.52 \\
\hline VANDERBILT & GLM+ & 320,134 & $7,611,939$ & $7,932,073$ & $11,063,708$ & 71.69 \\
\hline VIRGINIA & $\mathrm{LM}+$ & 420,967 & $4,929,132$ & $5,350,099$ & $13,107,062$ & 40.82 \\
\hline VIRGINIA TECH & G+ & 152,066 & $4,771,973$ & $4,924,039$ & $7,155,113$ & 68.82 \\
\hline WASHINGTON & BGLM+ & 580,828 & $8,091,885$ & $8,672,713$ & $16,657,209$ & 52.07 \\
\hline WASHINGTON STATE & $\mathrm{BbG}+$ & 71,416 & $4,165,990$ & $4,237,406$ & $6,152,879$ & 68.87 \\
\hline WASHINGTON U.-ST. LOUIS & GLM+ & 328,227 & $7,628,384$ & $7,956,611$ & $12,772,285$ & 62.30 \\
\hline WATERLOO & $\mathrm{G}^{+}$ & 140,103 & $3,696,597$ & $3,836,700$ & $5,925,083$ & 64.75 \\
\hline WAYNE STATE & GLM+ & 68,826 & $6,606,085$ & $6,674,911$ & $8,701,072$ & 76.71 \\
\hline WESTERN ONTARIO & GL+ & 41,451 & $6,237,740$ & $6,279,191$ & $10,185,101$ & 61.65 \\
\hline WISCONSIN & GLM+ & 838,788 & $5,675,154$ & $6,513,942$ & $11,710,988$ & 55.62 \\
\hline YALE & GLM+ & $1,830,705$ & $7,587,319$ & $9,418,024$ & $39,105,895$ & 24.08 \\
\hline YORK & BGL+ & 664,260 & $5,491,527$ & $6,155,786$ & $9,911,392$ & 62.11 \\
\hline BOSTON PUBLIC & $\mathrm{bG}+$ & UA/NA & UA/NA & . & $5,639,550$ & \\
\hline CENTER FOR RESEARCH LIBRARIES & & UA/NA & UA/NA & $\cdot$ & $1,271,497$ & \\
\hline LIBRARY OF CONGRESS & bG+ & $1,219,561$ & $2,632,675$ & $3,852,236$ & $19,376,303$ & 19.88 \\
\hline
\end{tabular}




\section{Electronic Resources ExPenditures}

Bibl. Utilities, Networks, etc. Bibl. Utilities, Networks, etc. Computer Hardware and Library Expenditures External Expenditures
Document Delivery/

Interlibrary Loan

Expenditures
(24a)
(24b)
(25)
(26) (Survey Question Number)

\begin{tabular}{|c|c|c|c|c|}
\hline 371,559 & UA/NA & $1,644,385$ & 241,568 & PENNSYLVANIA STATE \\
\hline $\mathrm{UA} / \mathrm{NA}$ & 288,467 & 683,558 & 202,572 & PITTSBURGH \\
\hline 322,771 & UA/NA & $1,391,759$ & 97,629 & PRINCETON \\
\hline 393,394 & 266,526 & 329,355 & 227,466 & PURDUE \\
\hline 0 & 448,976 & 200,756 & 28,337 & QUEEN'S \\
\hline 155,644 & 0 & 218,800 & 37,802 & RICE \\
\hline 172,426 & 0 & 852,814 & 68,146 & ROCHESTER \\
\hline 245,579 & UA/NA & 263,924 & 94,380 & RUTGERS \\
\hline 68,391 & 0 & 344,569 & 64,656 & SASKATCHEWAN \\
\hline 168,716 & 0 & 78,199 & 46,997 & SOUTH CAROLINA \\
\hline 526,269 & 0 & $2,136,968$ & 137,765 & SOUTHERN CALIFORNIA \\
\hline 214,231 & 58,732 & 498,524 & 120,154 & SOUTHERN ILLINOIS \\
\hline 0 & 78,299 & 122,145 & 132,480 & SUNY-ALBANY \\
\hline 225,642 & 0 & 305,031 & UA/NA & SUNY-BUFFALO \\
\hline 308,375 & UA/NA & 414,656 & 41,124 & SUNY-STONY BROOK \\
\hline UA/NA & UA/NA & 386,575 & 113,076 & SYRACUSE \\
\hline 129,150 & 9,000 & 780,523 & 155,517 & TEMPLE \\
\hline 28,469 & 31,569 & 658,717 & 167,121 & TENNESSEE \\
\hline 207,910 & 0 & $1,164,236$ & 308,594 & TEXAS \\
\hline 35,968 & 541,487 & $1,398,516$ & 424,840 & TEXAS A\&M \\
\hline 343,076 & 54,750 & $2,179,828$ & 86,812 & TEXAS TECH \\
\hline UA/NA & UA/NA & $3,071,239$ & 124,488 & TORONTO \\
\hline 207,711 & 93,920 & 210,525 & 11,657 & TULANE \\
\hline 295,637 & $1,995,021$ & $1,642,340$ & 44,793 & UTAH \\
\hline 254,816 & 0 & 673,584 & 94,843 & VANDERBILT \\
\hline 260,390 & 417,324 & $1,489,997$ & 213,094 & VIRGINIA \\
\hline 98,206 & 0 & 286,411 & 271,364 & VIRGINIA TECH \\
\hline 605,797 & 142,400 & 316,560 & 485,142 & WASHINGTON \\
\hline 138,950 & 74,975 & 310,845 & 36,673 & WASHINGTON STATE \\
\hline 290,397 & 811 & 771,591 & 30,631 & WASHINGTON U.-ST. LOUIS \\
\hline 13,316 & 0 & 313,742 & 55,930 & WATERLOO \\
\hline 137,370 & 0 & $1,756,977$ & 170,125 & WAYNE STATE \\
\hline 191,774 & 0 & 559,793 & 13,108 & WESTERN ONTARIO \\
\hline 370,454 & 412,445 & $2,039,511$ & 864,427 & WISCONSIN \\
\hline 246,362 & 0 & $1,374,190$ & 217,110 & YALE \\
\hline 139,338 & UA/NA & 342,570 & 12,970 & YORK \\
\hline UA/NA & UA/NA & UA/NA & $\mathrm{UA} / \mathrm{NA}$ & BOSTON PUBLIC \\
\hline 178,579 & $\cdot$ & 191,841 & 109,715 & CENTER FOR RESEARCH LIBRARIES \\
\hline 608,126 & 0 & $17,500,000$ & 11,000 & LIBRARY OF CONGRESS \\
\hline
\end{tabular}




\section{Electronic Resources Expenditures}

\begin{tabular}{|c|c|c|c|c|c|c|}
\hline & & $\begin{array}{r}\text { One-time } \\
\text { Electronic Resource } \\
\text { Purchases }\end{array}$ & $\begin{array}{r}\text { Ongoing } \\
\text { Electronic Resource } \\
\text { Purchases }\end{array}$ & $\begin{array}{r}\text { Total } \\
\text { Electronic Resource } \\
\text { Purchases }\end{array}$ & $\begin{array}{r}\text { Total } \\
\text { Library Materials } \\
\text { Expenditures }\end{array}$ & $\begin{array}{r}\text { Electronic Resources } \\
\text { as a \% of } \\
\text { Library Materials Budget }\end{array}$ \\
\hline (Survey Questio & n Number) & (22) & (23) & $(22+23)$ & (16) & \\
\hline & Notes & & & & & \\
\hline TURAL LIBRARY & G+ & 365,807 & $3,295,125$ & $3,660,932$ & $4,125,750$ & 88.73 \\
\hline S CANADA & $\mathrm{G}^{+}$ & . & 37,553 & 37,553 & $1,300,372$ & 2.89 \\
\hline OF MEDICINE & G+ & 1,049 & 224,305 & 225,354 & $9,421,417$ & 2.39 \\
\hline & + & $\mathrm{UA} / \mathrm{NA}$ & $4,478,033$ & $4,478,033$ & $12,658,812$ & 35.37 \\
\hline & G+ & 0 & 432,093 & 432,093 & $3,436,483$ & 12.57 \\
\hline & G+ & 0 & 265,033 & 265,033 & $1,543,521$ & 17.17 \\
\hline
\end{tabular}

+ - See Footnotes

L - Includes Law library

M- Includes Medical library

\author{
G - Government documents not included in serials count \\ $\mathrm{B}$ - Includes branch campuses \\ $\mathrm{b}$ - Basis of volume count is bibliographic
}

U / A - Unavailable

N/A - Not Applicable

. - no data supplied

\section{Summary Data}

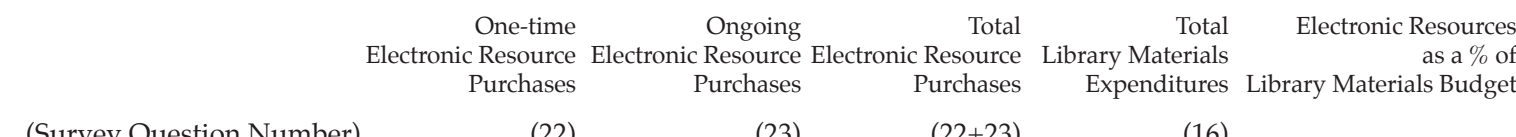

(Survey Question Number)

(22)

(23)

$(22+23)$

(16)

\section{University Libraries}

Mean
Median
High
Low
Totals
Number of Libraries Reporting
Muniversity Libraries
Median
Totals
Number of Libraries Reporting

GRAND TOTALS

$\begin{array}{rr}628,620 & 5,691,593 \\ 363,746 & 5,337,237 \\ 10,204,304 & 10,568,113 \\ 1,725 & 1,508,822 \\ 69,148,203 & 637,458,376 \\ 110 & 112\end{array}$

$\begin{array}{rr}6,253,156 & 11,596,797 \\ 5,854,147 & 10,274,939 \\ 14,314,075 & 39,105,895 \\ 1,530,825 & 4,073,010 \\ 706,606,579 & 1,322,034,898 \\ 113 & 114\end{array}$

56.33

57.03

85.40

\section{Nonuniversity Libraries}

Median

$\begin{array}{rr}1,049 & 432,093 \\ 1,586,417 & 11,364,817 \\ 5 & 7\end{array}$

$$
432,093
$$

$4,125,750$

$12,951,234$

$58,773,705$ 


\section{Electronic Resources Expenditures}

Bibl. Utilities, Networks, etc. Bibl. Utilities, Networks, etc. Library Expenditures
Computer Hardware and Software Expenditures
Document Delivery/ Interlibrary Loan Expenditures (24a)

(24b)
(25)
(26) (Survey Question Number)

INSTITUTION

\begin{tabular}{rrrrr}
\hline 41,142 & 0 & 230,549 & 487,538 & NATIONAL AGRICULTURAL LIBRARY \\
$\cdot$ & $\cdot$ & $8,478,606$ & 9,685 & LIBRARY \& ARCHIVES CANADA \\
$\cdot$ & $\cdot$ &. &. & NATIONAL LIBRARY OF MEDICINE \\
288,605 & UA/NA & $1,964,470$ & UA/NA & NEW YORK PUBLIC \\
55,408 & 0 & 0 & UA/NA & NEW YORK STATE \\
51,588 & 0 & 50,992 & 564 & SMITHSONIAN
\end{tabular}

\section{Summary Data}

Bibl. Utilities, Networks, etc. Library Expenditures
Bibl.Utilities, Networks, etc External Expenditures
Computer Hardware and Software Expenditures
Document Delivery/ Interlibrary Loan Expenditures (24a)

(24b)
(26) (Survey Question Number)

University Libraries

Mean

Median

$472,267 \quad 104,708$

$3,071,239 \quad 900,893$

$21,020 \quad 603$

$77,239,838$

$16,327,690$

111

High

Low

Totals

Number of Libraries Reporting

Nonuniversity Libraries

Median

Totals

$28,416,458 \quad 618,502$

5

Number of Libraries Reporting 


\section{Personnel and Public Services}

Professional Staff Support Staff Student Assistants Total Staff (FTE)

(Survey Question Number)

(FTE)

(27a)

(27b)
$(27 c)$
Notes

\begin{tabular}{|c|c|c|c|c|c|c|c|}
\hline INSTITUTION & Notes & & & & & & \\
\hline ALABAMA & bGLM+ & 73 & 77 & 53 & 203 & 20 & 136 \\
\hline ALBERTA & GLM+ & 88 & 214 & 34 & 336 & 19 & 103 \\
\hline ARIZONA & bGLM+ & 91 & 123 & 73 & 287 & 18 & 142 \\
\hline ARIZONA STATE & BGL+ & 91 & 171 & 78 & 340 & 18 & 168 \\
\hline AUBURN & + & 45 & 45 & 33 & 123 & 11 & 134 \\
\hline BOSTON & $\mathrm{LM}+$ & 104 & 96 & 81 & 281 & 32 & 108 \\
\hline BOSTON COLLEGE & $\mathrm{L}+$ & 75 & 72 & 43 & 190 & 18 & 110 \\
\hline BRIGHAM YOUNG & GL+ & 126 & 59 & 209 & 394 & 18 & 105 \\
\hline BRITISH COLUMBIA & LM+ & 123 & 178 & 38 & 339 & 31 & 101 \\
\hline BROWN & bGM+ & 77 & 99 & 24 & 200 & 8 & 112 \\
\hline CALGARY & GLM+ & 71 & 168 & 28 & 267 & 22 & 124 \\
\hline CALIFORNIA, BERKELEY & $\mathrm{L}+$ & 243 & 201 & 146 & 590 & 49 & 118 \\
\hline CALIFORNIA, DAVIS & GLM+ & 55 & 122 & 31 & 208 & 13 & 95 \\
\hline CALIFORNIA, IRVINE & bGM+ & 69 & 121 & 40 & 230 & 14 & 128 \\
\hline CALIFORNIA, LOS ANGELES & bGLM+ & 163 & 272 & 145 & 580 & 30 & 96 \\
\hline CALIFORNIA, RIVERSIDE & G+ & 45 & 81 & 24 & 150 & 13 & 94 \\
\hline CALIFORNIA, SAN DIEGO & bGM+ & 110 & 188 & 63 & 361 & 19 & 115 \\
\hline CALIFORNIA, SANTA BARBARA & G+ & 47 & 118 & 45 & 210 & 10 & 103 \\
\hline CASE WESTERN RESERVE & GLM+ & 59 & 45 & 30 & 134 & 18 & 104 \\
\hline CHICAGO & bGLM+ & 70 & 181 & 63 & 314 & 30 & 146 \\
\hline CINCINNATI & GLM+ & 78 & 51 & 47 & 176 & 19 & 103 \\
\hline COLORADO & bG+ & 56 & 115 & 47 & 218 & 12 & 104 \\
\hline COLORADO STATE & G+ & 48 & 51 & 24 & 123 & 7 & 108 \\
\hline COLUMBIA & GLM+ & 311 & 225 & 121 & 657 & 47 & 108 \\
\hline CONNECTICUT & BbGLM+ & 85 & 67 & 46 & 198 & 19 & 114 \\
\hline CORNELL & BGL+ & 118 & 286 & 120 & 524 & 43 & 146 \\
\hline DARTMOUTH & bGM+ & 55 & 113 & 28 & 196 & 20 & 118 \\
\hline DELAWARE & $\mathrm{G}^{+}$ & 58 & 94 & 37 & 189 & 15 & 100 \\
\hline DUKE & $\mathrm{LM}+$ & 183 & 131 & 34 & 348 & 18 & 155 \\
\hline EMORY & BLM+ & 93 & 155 & 35 & 283 & 27 & 112 \\
\hline FLORIDA & $\mathrm{BbLM}+$ & 98 & 180 & 209 & 487 & 19 & 105 \\
\hline FLORIDA STATE & BbLM+ & 62 & 98 & 64 & 224 & 21 & 105 \\
\hline GEORGE WASHINGTON & BGLM+ & 85 & 108 & 52 & 245 & 21 & 168 \\
\hline GEORGETOWN & bGLM+ & 99 & 114 & 48 & 261 & 20 & 107 \\
\hline GEORGIA & GL+ & 79 & 171 & 36 & 286 & 22 & 137 \\
\hline GEORGIA TECH & $\mathrm{G}^{+}$ & 46 & 69 & 8 & 123 & 8 & 135 \\
\hline GUELPH & BG+ & 51 & 69 & 16 & 136 & 12 & 116 \\
\hline HARVARD & GLM+ & 565 & 529 & 120 & 1,214 & UA/NA & $\mathrm{UA} / \mathrm{NA}$ \\
\hline HAWAII & GLM+ & 90 & 81 & 67 & 238 & 16 & 168 \\
\hline
\end{tabular}

(FTE) (FTE)
Staffed

Service

Points

(28)
Library

Service

Hours

(29) 


\section{Personnel and Public Services}

\begin{tabular}{|c|c|c|c|c|c|c|}
\hline $\begin{array}{r}\text { Library } \\
\text { Presentations } \\
\text { to Groups }\end{array}$ & $\begin{array}{r}\text { Participants } \\
\text { in Group } \\
\text { Presentations }\end{array}$ & $\begin{array}{r}\text { Reference } \\
\text { Transactions }\end{array}$ & $\begin{array}{r}\text { Initial } \\
\text { Circulation } \\
\text { Transactions }\end{array}$ & $\begin{array}{r}\text { Total } \\
\text { Circulation } \\
\text { Transactions }\end{array}$ & $\begin{array}{r}\text { Total Items } \\
\text { Loaned } \\
\text { (ILL) }\end{array}$ & $\begin{array}{r}\text { Total Items } \\
\text { Borrowed } \\
\text { (ILL) }\end{array}$ \\
\hline (30) & (31) & (32) & (33) & (34) & (35) & (36) (Survey Question Number) \\
\hline
\end{tabular}

INSTITUTION

\begin{tabular}{|c|c|c|c|c|c|c|c|}
\hline 431 & 8,069 & 61,258 & 231,737 & 265,821 & 11,983 & 11,523 & ALABAMA \\
\hline 811 & 19,619 & 92,323 & 459,206 & 735,620 & 49,147 & 17,670 & ALBERTA \\
\hline 2,337 & 26,473 & 42,620 & 300,050 & 460,779 & 58,338 & 45,608 & ARIZONA \\
\hline 1,111 & 23,515 & 92,462 & 292,266 & 864,474 & 51,378 & 44,348 & ARIZONA STATE \\
\hline 780 & 15,253 & 100,568 & 164,246 & 194,683 & 13,542 & 10,265 & AUBURN \\
\hline 1,246 & 19,700 & 44,068 & 177,224 & 203,182 & 19,710 & 21,321 & BOSTON \\
\hline 643 & 13,996 & 18,660 & 192,677 & 303,974 & 26,599 & 19,577 & BOSTON COLLEGE \\
\hline 2,158 & 42,529 & 126,184 & 459,907 & 609,610 & 62,169 & 32,332 & BRIGHAM YOUNG \\
\hline 1,421 & 32,440 & 100,288 & 659,810 & $2,513,529$ & 23,978 & 16,602 & BRITISH COLUMBIA \\
\hline 538 & 6,864 & 13,278 & 197,117 & 289,241 & 37,189 & 39,355 & BROWN \\
\hline 812 & 19,688 & 76,362 & 261,993 & $1,232,050$ & 19,016 & 27,506 & CALGARY \\
\hline 1,757 & 28,120 & 77,126 & 696,008 & 866,063 & 37,362 & 26,202 & CALIFORNIA, BERKELEY \\
\hline 853 & 12,958 & 100,831 & 277,239 & 518,884 & 41,383 & 24,345 & CALIFORNIA, DAVIS \\
\hline 697 & 19,951 & 62,610 & 153,586 & 343,272 & 26,281 & 28,187 & CALIFORNIA, IRVINE \\
\hline 1,245 & 24,977 & 126,716 & 663,785 & $2,165,844$ & 46,796 & 37,922 & CALIFORNIA, LOS ANGELES \\
\hline 783 & 13,230 & 26,524 & 124,184 & 184,580 & 36,452 & 25,623 & CALIFORNIA, RIVERSIDE \\
\hline 1,105 & 12,777 & 100,623 & 439,284 & 661,746 & 31,545 & 27,976 & CALIFORNIA, SAN DIEGO \\
\hline 696 & 9,594 & 75,036 & 217,529 & 354,016 & 23,207 & 36,918 & CALIFORNIA, SANTA BARBARA \\
\hline 642 & 8,579 & 19,577 & 91,470 & 162,620 & 33,485 & 35,459 & CASE WESTERN RESERVE \\
\hline 464 & 7,262 & 25,952 & 322,083 & 613,425 & 56,493 & 18,824 & CHICAGO \\
\hline 1,003 & 19,655 & 68,998 & 228,188 & 344,150 & 59,462 & 40,755 & CINCINNATI \\
\hline 676 & 13,054 & 70,753 & 326,128 & 558,966 & 55,834 & 32,948 & COLORADO \\
\hline 454 & 10,354 & 68,848 & 190,981 & 332,063 & 81,331 & 65,209 & COLORADO STATE \\
\hline 1,490 & 23,119 & 171,600 & 412,278 & 882,708 & 46,134 & 55,437 & COLUMBIA \\
\hline 1,371 & 19,862 & 31,878 & 158,502 & 173,838 & 40,370 & 48,491 & CONNECTICUT \\
\hline 1,835 & 23,372 & 70,639 & 413,096 & 862,171 & 58,351 & 33,826 & CORNELL \\
\hline UA/NA & $\mathrm{UA} / \mathrm{NA}$ & UA/NA & 266,277 & 349,207 & 41,775 & 31,634 & DARTMOUTH \\
\hline 1,519 & 8,146 & 131,284 & $\mathrm{UA} / \mathrm{NA}$ & 398,285 & 18,700 & 24,559 & DELAWARE \\
\hline 1,041 & 12,979 & 131,763 & 465,947 & 681,640 & 35,589 & 29,378 & DUKE \\
\hline 1,208 & 19,210 & 47,319 & 219,979 & 559,382 & 36,890 & 18,776 & EMORY \\
\hline 459 & 11,184 & 55,416 & 408,124 & 801,338 & 18,238 & 15,899 & FLORIDA \\
\hline 859 & 18,684 & 89,129 & 249,774 & 366,131 & 23,607 & 13,854 & FLORIDA STATE \\
\hline 1,380 & 21,016 & 36,561 & 178,489 & 293,139 & 31,907 & 57,876 & GEORGE WASHINGTON \\
\hline 1,076 & 13,141 & 66,013 & 302,438 & 405,307 & 35,259 & 37,418 & GEORGETOWN \\
\hline 949 & 16,234 & 46,464 & 266,206 & 423,657 & 40,706 & 7,861 & GEORGIA \\
\hline 351 & 9,447 & 13,520 & 118,972 & $\mathrm{UA} / \mathrm{NA}$ & 12,694 & 12,305 & GEORGIA TECH \\
\hline 340 & 7,838 & 12,310 & 144,540 & 390,967 & 44,136 & 46,607 & GUELPH \\
\hline UA/NA & UA/NA & UA/NA & $\mathrm{UA} / \mathrm{NA}$ & $2,023,014$ & 39,760 & 14,594 & HARVARD \\
\hline 539 & 9,239 & 48,037 & 226,230 & 428,949 & 6,843 & 30,273 & HAWAII \\
\hline
\end{tabular}


Personnel and Public Services

Professional Staff Support Staff Student Assistants Total Staff (FTE)

(FTE)

(FTE)

(FTE)

(Survey Question Number)

(27a)

$(27 b)$

$(27 c)$

(27)

Staffed

Service

Points

Library

Service

Hours

\section{INSTITUTION}

Notes

HOUSTON

HOWARD

bL+

ILLINOIS, CHICAGO

ILLINOIS, URBANA

bLM+

61

38

$\mathrm{M}+$

INDIANA

bGL+

65
191

IOWA

IOWA STATE

L+

$\mathrm{LM}+$

JOHNS HOPKINS

KANSAS

KENT STATE

KENTUCKY

G+

104

LAVAL

LOUISIANA STATE

LOUISVILLE

MCGILL

MCMASTER

MANITOBA

$\mathrm{BbGM}+$

BbGLM+

\section{$\mathrm{BbG}+$}

GLM+

GLM+

\section{GLM+}

BGM+

BbGLM+

MARYLAND

MASSACHUSETTS

MIT

MIAMI

MICHIGAN

MICHIGAN STATE

MINNESOTA

MISSOURI

MONTREAL

bGM+

GLM+

G+

G+

57

77

77

57

93

$191 \quad 200$

135

$104 \quad 117$

52

124

103

$55 \quad 40$

$87 \quad 117$

61

$61 \quad 154$

$51 \quad 79$

$51 \quad 93$

$77 \quad 147$

$57 \quad 8$

$63 \quad 135$

121

59

96

G+

bGLM+ 83

GLM+ 167

GL+ 78

bGLM+

112

GLM+

GLM+

56

NEBRASKA

L+

NEW MEXICO

NEW YORK

NORTH CAROLINA

NORTH CAROLINA STATE

NORTHWESTERN

GLM+

bGLM+

GLM+

$\mathrm{G}+$

BGLM+

56
114

53

$53 \quad 102$

$60 \quad 130$

$148 \quad 226$

158

129

121

$82 \quad 143$

NOTRE DAME

OHIO

OHIO STATE

bGL+

BbGM+

bGLM+

48

48
172

GLM+

$\begin{array}{rr}61 & 96 \\ 66 & 79 \\ 72 & 82 \\ 136 & 176\end{array}$

BGM+

BGL+

GLM+

PENNSYLVANIA

136

176

154
79
93

$\begin{array}{rrrr}50 & 188 & 8 & 108 \\ 62 & 157 & 16 & 113 \\ 27 & 185 & 27 & 102 \\ 122 & 513 & 35 & 144 \\ 115 & 415 & 57 & 168\end{array}$

$\begin{array}{llll}61 & 282 & 24 & 115\end{array}$

$\begin{array}{llll}30 & 167 & 9 & 105\end{array}$

$\begin{array}{llll}40 & 299 & 29 & 120\end{array}$

$\begin{array}{llll}75 & 291 & 10 & 142\end{array}$

$\begin{array}{llll}15 & 110 & 13 & 99\end{array}$

$\begin{array}{llll}64 & 268 & 20 & 140\end{array}$

$\begin{array}{rrrr}1 & 216 & 18 & 90 \\ 57 & 187 & 14 & 109\end{array}$

$\begin{array}{llll}39 & 183 & 11 & 97\end{array}$

26

$81 \quad 30$

$35 \quad 18$

$96 \quad 51$

74

$51 \quad 2$

189

$\begin{array}{llll}18 & 206 & 11 & 104\end{array}$

$\begin{array}{rrrr}65 & 276 & 23 & 122 \\ 108 & 584 & 57 & 168\end{array}$

$\begin{array}{llll}74 & 273 & 20 & 148\end{array}$

$\begin{array}{llll}76 & 391 & 46 & 100 \\ 33 & 190 & 26 & 114\end{array}$

$\begin{array}{llll}3 & 365 & 66 & 97\end{array}$

$\begin{array}{rrrr}42 & 197 & 25 & 96 \\ 45 & 235 & 18 & 133 \\ 97 & 471 & 34 & 126\end{array}$

$\begin{array}{llll}97 & 471 & 34 & 133\end{array}$

$\begin{array}{llll}96 & 468 & 25 & 142 \\ 38 & 272 & 14 & 146\end{array}$

$\begin{array}{llll}83 & 343 & 29 & 14\end{array}$

$\begin{array}{llll}44 & 269 & 18 & 126 \\ 45 & 161 & 15 & 146\end{array}$

$\begin{array}{llll}150 & 161 & 15 & 146 \\ 76 & 469 & 21 & 168\end{array}$

$\begin{array}{rlll}150 & 469 & 21 & 168 \\ 76 & 233 & 22 & 117 \\ 72 & 217 & 10 & 115 \\ 63 & 217 & 20 & 106\end{array}$

$\begin{array}{llll}72 & 217 & 10 & 115 \\ 63 & 217 & 20 & 106\end{array}$

$\begin{array}{llll}63 & 217 & 20 & 106 \\ 93 & 405 & 38 & 116\end{array}$




\section{Personnel and Public Services}

\begin{tabular}{|c|c|c|c|c|c|c|}
\hline $\begin{array}{r}\text { Library } \\
\text { Presentations } \\
\text { to Groups }\end{array}$ & $\begin{array}{r}\text { Participants } \\
\text { in Group } \\
\text { Presentations }\end{array}$ & $\begin{array}{r}\text { Reference } \\
\text { Transactions }\end{array}$ & $\begin{array}{r}\text { Initial } \\
\text { Circulation } \\
\text { Transactions }\end{array}$ & $\begin{array}{r}\text { Total } \\
\text { Circulation } \\
\text { Transactions }\end{array}$ & $\begin{array}{r}\text { Total Items } \\
\text { Loaned } \\
\text { (ILL) }\end{array}$ & $\begin{array}{r}\text { Total Items } \\
\text { Borrowed } \\
\text { (ILL) }\end{array}$ \\
\hline (30) & (31) & (32) & (33) & (34) & (35) & (36) (Survey Question Number) \\
\hline
\end{tabular}

(33)

(34)

(35)

(36) (Survey Question Number)

\begin{tabular}{|c|c|c|c|c|c|c|c|}
\hline 414 & 8,847 & 24,966 & 112,545 & 190,612 & 24,690 & 16,771 & $\overline{\text { HOUSTON }}$ \\
\hline 303 & 6,241 & 42,389 & 43,661 & 56,984 & 7,872 & 4,764 & HOWARD \\
\hline 875 & 15,216 & 63,448 & 140,865 & 437,385 & 64,997 & 53,004 & ILLINOIS, CHICAGO \\
\hline 1,503 & 28,196 & 130,136 & 524,085 & $1,024,562$ & 91,700 & 78,564 & ILLINOIS, URBANA \\
\hline 1,902 & 35,949 & 94,557 & 682,367 & 777,034 & 45,546 & 46,885 & INDIANA \\
\hline 1,053 & 17,527 & 60,772 & 297,587 & 369,294 & 59,427 & 33,684 & IOWA \\
\hline 362 & 11,628 & 23,844 & 202,833 & 251,683 & 34,033 & 17,754 & IOWA STATE \\
\hline 1,208 & 13,538 & 40,423 & 259,981 & 442,834 & 45,350 & 43,247 & JOHNS HOPKINS \\
\hline 1,320 & 20,581 & 100,542 & 341,170 & 578,728 & 49,923 & 39,196 & KANSAS \\
\hline 426 & 8,351 & 20,703 & 210,058 & 334,049 & 14,062 & 8,579 & KENT STATE \\
\hline 769 & 8,352 & 38,347 & 178,648 & 227,957 & 30,301 & 26,164 & KENTUCKY \\
\hline 474 & 12,346 & 138,253 & 504,243 & 780,144 & 18,724 & 9,154 & LAVAL \\
\hline 974 & 6,884 & 32,017 & 120,381 & 221,513 & 16,593 & 16,231 & LOUISIANA STATE \\
\hline 659 & 11,344 & 61,438 & $\mathrm{UA} / \mathrm{NA}$ & 662,055 & 24,753 & 28,640 & LOUISVILLE \\
\hline 1,743 & 21,511 & 225,445 & 481,226 & 805,882 & 20,387 & 14,108 & MCGILL \\
\hline 673 & 18,166 & 38,738 & 228,825 & 382,786 & 15,394 & 14,519 & MCMASTER \\
\hline 1,197 & 13,948 & 95,782 & 212,788 & 386,639 & 18,981 & 24,583 & MANITOBA \\
\hline 1,033 & 21,512 & 320,221 & 278,158 & 496,128 & 25,268 & 39,117 & MARYLAND \\
\hline 499 & 9,811 & 42,742 & 180,319 & 296,062 & 37,532 & 44,014 & MASSACHUSETTS \\
\hline 309 & 7,075 & 26,972 & 183,414 & 334,336 & 18,086 & 20,096 & MIT \\
\hline 2,239 & 22,900 & 61,436 & 161,790 & 233,800 & 34,202 & 13,807 & MIAMI \\
\hline 1,423 & 26,880 & 145,279 & 558,879 & $1,146,480$ & 52,862 & 52,435 & MICHIGAN \\
\hline 970 & 39,286 & 47,047 & 291,748 & 635,208 & 68,911 & 33,983 & MICHIGAN STATE \\
\hline 1,648 & 31,324 & 84,874 & 361,318 & 699,640 & 178,255 & 41,710 & MINNESOTA \\
\hline 886 & 11,425 & 40,944 & 196,433 & 263,123 & 59,060 & 43,809 & MISSOURI \\
\hline 1,117 & 17,849 & 77,150 & 406,598 & 841,480 & 27,126 & 19,824 & MONTREAL \\
\hline 242 & 6,212 & 63,163 & 177,435 & 290,386 & 32,166 & 31,139 & NEBRASKA \\
\hline 1,462 & 19,505 & 35,727 & 184,020 & 257,138 & 25,031 & 32,666 & NEW MEXICO \\
\hline 1,240 & 19,140 & 122,952 & 483,943 & 852,751 & 25,135 & 39,993 & NEW YORK \\
\hline 1,551 & 29,094 & 117,602 & 721,078 & 971,595 & 66,072 & 19,011 & NORTH CAROLINA \\
\hline 417 & 11,092 & 32,585 & 324,709 & 453,045 & 19,495 & 27,229 & NORTH CAROLINA STATE \\
\hline 982 & 12,720 & 65,886 & 202,862 & 317,174 & 37,518 & 34,168 & NORTHWESTERN \\
\hline 398 & 6,728 & 21,333 & 200,406 & 351,649 & 25,342 & 24,898 & NOTRE DAME \\
\hline 753 & 14,028 & 59,457 & 307,125 & 500,176 & 91,863 & 74,464 & OHIO \\
\hline 1,679 & 29,644 & 20,176 & 431,715 & $1,487,883$ & 148,084 & 96,431 & OHIO STATE \\
\hline 1,254 & 11,515 & 45,514 & 282,614 & 299,442 & 42,185 & 32,889 & OKLAHOMA \\
\hline 1,111 & 30,021 & 122,848 & 294,088 & 347,573 & 31,401 & 23,836 & OKLAHOMA STATE \\
\hline 703 & 11,925 & 50,761 & 208,918 & 280,635 & 67,678 & 58,336 & OREGON \\
\hline 1,228 & 21,546 & $\mathrm{UA} / \mathrm{NA}$ & 314,780 & 451,792 & 70,875 & 93,481 & PENNSYLVANIA \\
\hline
\end{tabular}




\section{Personnel and Public Services}

Professional Staff Support Staff Student Assistants Total Staff (FTE)

(FTE)

(FTE)

(FTE)

Staffed

Service

Library

Points

Service

(Survey Question Number)

(27a)

(27b)

(27c)

(27)

(28)

(29)

\begin{tabular}{|c|c|c|c|c|c|c|c|}
\hline INSTITUTION & Notes & & & & & & \\
\hline PENNSYLVANIA STATE & BbGLM+ & 161 & 370 & 65 & 596 & 63 & 168 \\
\hline PITTSBURGH & BGLM+ & 121 & 171 & 61 & 353 & 45 & 118 \\
\hline PRINCETON & bG+ & 143 & 215 & 47 & 405 & 37 & 117 \\
\hline PURDUE & G+ & 64 & 102 & 55 & 221 & 16 & 115 \\
\hline QUEEN'S & $\mathrm{LM}+$ & 44 & 108 & 18 & 170 & 13 & 115 \\
\hline RICE & G+ & 59 & 50 & 10 & 119 & 12 & 143 \\
\hline ROCHESTER & $\mathrm{bM}+$ & 101 & 59 & 55 & 215 & 21 & 119 \\
\hline RUTGERS & BGL+ & 90 & 235 & 81 & 406 & 33 & 109 \\
\hline SASKATCHEWAN & GLM+ & 51 & 88 & 15 & 154 & 17 & 95 \\
\hline SOUTH CAROLINA & $\mathrm{LM}+$ & 72 & 103 & 99 & 274 & 19 & 111 \\
\hline SOUTHERN CALIFORNIA & BGLM+ & 129 & 140 & 104 & 373 & 29 & 160 \\
\hline SOUTHERN ILLINOIS & $\mathrm{LM}+$ & 52 & 91 & 48 & 191 & 13 & 107 \\
\hline SUNY-ALBANY & G+ & 72 & 49 & 30 & 151 & 10 & 108 \\
\hline SUNY-BUFFALO & GLM+ & 102 & 66 & 49 & 217 & 15 & 168 \\
\hline SUNY-STONY BROOK & $\mathrm{bM}+$ & 86 & 26 & 32 & 144 & 12 & 109 \\
\hline SYRACUSE & GL+ & 62 & 95 & 34 & 191 & 18 & 106 \\
\hline TEMPLE & BGLM+ & 75 & 92 & 46 & 213 & 16 & 108 \\
\hline TENNESSEE & BbGLM+ & 87 & 125 & 37 & 249 & 17 & 148 \\
\hline TEXAS & GL+ & 149 & 289 & 122 & 560 & 34 & 120 \\
\hline TEXAS A\&M & $\mathrm{BbG}+$ & 147 & 131 & 77 & 355 & 17 & 145 \\
\hline TEXAS TECH & BLM+ & 103 & 137 & 97 & 337 & 31 & 130 \\
\hline TORONTO & BLM+ & 171 & 343 & 187 & 701 & 90 & 168 \\
\hline TULANE & BGLM+ & 45 & 84 & 24 & 153 & 22 & 168 \\
\hline UTAH & $\mathrm{LM}+$ & 84 & 157 & 82 & 323 & 17 & 108 \\
\hline VANDERBILT & GLM+ & 108 & 102 & 34 & 244 & 15 & 146 \\
\hline VIRGINIA & $\mathrm{LM}+$ & 104 & 206 & 68 & 378 & 30 & 149 \\
\hline VIRGINIA TECH & G+ & 38 & 83 & 24 & 145 & 9 & 102 \\
\hline WASHINGTON & BGLM+ & 194 & 199 & 128 & 521 & 45 & 138 \\
\hline WASHINGTON STATE & $\mathrm{BbG+}$ & 52 & 83 & 35 & 170 & 19 & 100 \\
\hline WASHINGTON U.-ST. LOUIS & GLM+ & 91 & 115 & 52 & 258 & 17 & 120 \\
\hline WATERLOO & G+ & 38 & 91 & 35 & 164 & 14 & 106 \\
\hline WAYNE STATE & GLM+ & 101 & 81 & 64 & 246 & 15 & 142 \\
\hline WESTERN ONTARIO & GL+ & 70 & 106 & 15 & 191 & 8 & 107 \\
\hline WISCONSIN & GLM+ & 245 & 165 & 197 & 607 & 45 & 148 \\
\hline YALE & GLM+ & 266 & 378 & 72 & 716 & 38 & 111 \\
\hline YORK & BGL+ & 62 & 110 & 60 & 232 & 20 & 141 \\
\hline BOSTON PUBLIC & $\mathrm{bG}+$ & 155 & 292 & 0 & 447 & 96 & 168 \\
\hline CENTER FOR RESEARCH LIBRARIES & & 31 & 16 & 12 & 59 & 2 & 35 \\
\hline LIBRARY OF CONGRESS & bG+ & 2,937 & 687 & 50 & 3,674 & 38 & 65 \\
\hline
\end{tabular}




\section{Personnel and Public Services}

\begin{tabular}{|c|c|c|c|c|c|c|}
\hline $\begin{array}{r}\text { Library } \\
\text { Presentations } \\
\text { to Groups }\end{array}$ & $\begin{array}{r}\text { Participants } \\
\text { in Group } \\
\text { Presentations }\end{array}$ & $\begin{array}{r}\text { Reference } \\
\text { Transactions }\end{array}$ & $\begin{array}{r}\text { Initial } \\
\text { Circulation } \\
\text { Transactions }\end{array}$ & $\begin{array}{r}\text { Total } \\
\text { Circulation } \\
\text { Transactions }\end{array}$ & $\begin{array}{r}\text { Total Items } \\
\text { Loaned } \\
\text { (ILL) }\end{array}$ & $\begin{array}{r}\text { Total Items } \\
\text { Borrowed } \\
\text { (ILL) }\end{array}$ \\
\hline (30) & (31) & (32) & (33) & (34) & (35) & (36) (Survey Question Number) \\
\hline
\end{tabular}

(34)

INSTITUTION

\begin{tabular}{|c|c|c|c|c|c|c|c|}
\hline 2,832 & 56,172 & 192,816 & 520,557 & 926,253 & 75,432 & 54,445 & PENNSYLVANIA STATE \\
\hline 1,236 & 23,894 & 123,848 & 349,666 & 476,989 & 76,898 & 28,180 & PITTSBURGH \\
\hline 735 & 12,117 & 22,076 & 285,238 & 389,713 & 47,441 & 35,857 & PRINCETON \\
\hline 375 & 11,874 & 46,321 & 128,173 & 340,885 & 28,638 & 44,428 & PURDUE \\
\hline 831 & 18,672 & 28,749 & 213,324 & 506,648 & 17,610 & 7,168 & QUEEN'S \\
\hline 175 & 2,980 & $\mathrm{UA} / \mathrm{NA}$ & 148,643 & 246,616 & 13,437 & 10,841 & RICE \\
\hline 599 & 9,949 & 53,552 & 195,690 & 228,903 & 22,571 & 25,064 & ROCHESTER \\
\hline 1,042 & 21,680 & 89,963 & 257,357 & 543,728 & 45,230 & 35,774 & RUTGERS \\
\hline 506 & 8,632 & 18,945 & 178,725 & 257,792 & 16,113 & 19,847 & SASKATCHEWAN \\
\hline 674 & 10,508 & 109,344 & $\mathrm{UA} / \mathrm{NA}$ & 254,624 & 17,142 & 17,391 & SOUTH CAROLINA \\
\hline 1,070 & 17,905 & 84,616 & 300,799 & 514,036 & 24,473 & 20,439 & SOUTHERN CALIFORNIA \\
\hline 870 & 10,163 & 33,043 & 232,430 & 363,588 & 51,209 & 41,563 & SOUTHERN ILLINOIS \\
\hline 590 & 6,038 & 62,930 & 86,235 & 142,296 & 12,428 & 22,371 & SUNY-ALBANY \\
\hline 887 & 30,163 & 19,450 & 207,750 & 463,188 & 26,996 & 23,676 & SUNY-BUFFALO \\
\hline 530 & 10,075 & 34,441 & 164,823 & 174,791 & 21,195 & 11,824 & SUNY-STONY BROOK \\
\hline 577 & 11,064 & 38,062 & 166,171 & 258,463 & 12,427 & 21,452 & SYRACUSE \\
\hline 963 & 18,670 & 66,064 & 220,278 & 300,043 & 29,292 & 26,406 & TEMPLE \\
\hline 870 & 16,668 & 107,808 & 256,103 & 317,801 & 34,534 & 21,944 & TENNESSEE \\
\hline 1,761 & 43,285 & 130,458 & $1,442,098$ & $2,234,203$ & 61,799 & 28,329 & TEXAS \\
\hline 771 & 28,716 & 38,208 & $1,245,397$ & $1,918,964$ & 45,649 & 52,687 & TEXAS A\&M \\
\hline 1,949 & 23,378 & 36,198 & 238,858 & 330,797 & 35,890 & 32,012 & TEXAS TECH \\
\hline 3,007 & 54,552 & 328,421 & $1,196,893$ & $1,729,144$ & 30,099 & 9,119 & TORONTO \\
\hline 358 & 5,035 & 29,326 & 113,801 & 157,852 & 14,929 & 11,192 & TULANE \\
\hline 1,711 & 29,908 & 192,587 & 233,872 & 540,117 & 42,479 & 37,036 & UTAH \\
\hline 750 & 16,182 & 23,954 & 197,978 & 330,902 & 25,748 & 16,505 & VANDERBILT \\
\hline 1,286 & 13,414 & 105,965 & 490,766 & 877,168 & 37,176 & 32,075 & VIRGINIA \\
\hline 664 & 14,700 & 19,021 & 238,989 & 353,516 & 20,010 & 20,705 & VIRGINIA TECH \\
\hline 1,294 & 25,613 & 118,794 & 672,309 & $1,654,212$ & 95,895 & 97,626 & WASHINGTON \\
\hline 875 & 19,452 & 43,155 & 140,932 & 259,101 & 36,420 & 23,105 & WASHINGTON STATE \\
\hline 878 & 8,163 & 59,161 & 226,324 & 435,800 & 59,715 & 51,249 & WASHINGTON U.-ST. LOUIS \\
\hline 174 & 6,291 & 20,665 & 205,368 & 554,609 & 50,116 & 46,099 & WATERLOO \\
\hline 428 & 8,940 & 38,684 & 91,130 & 140,732 & 48,424 & 42,671 & WAYNE STATE \\
\hline 847 & 18,006 & 28,858 & 381,275 & 657,609 & 13,138 & 9,613 & WESTERN ONTARIO \\
\hline 1,569 & 27,444 & $\mathrm{UA} / \mathrm{NA}$ & 511,692 & 790,184 & 90,581 & 95,513 & WISCONSIN \\
\hline 1,832 & 15,515 & 72,383 & 458,355 & 884,996 & 46,497 & 42,194 & YALE \\
\hline 749 & 25,508 & 117,908 & 439,082 & 963,555 & 15,335 & 5,554 & YORK \\
\hline 9,834 & 181,923 & $1,122,186$ & $3,403,538$ & $3,403,538$ & 20,009 & 17,535 & BOSTON PUBLIC \\
\hline 38 & 1,109 & & 79,060 & 79,060 & 31,325 & 0 & CENTER FOR RESEARCH LIBRARIES \\
\hline 5,740 & 186,935 & 591,784 & $1,132,564$ & $\mathrm{UA} / \mathrm{NA}$ & 34,073 & $\mathrm{UA} / \mathrm{NA}$ & LIBRARY OF CONGRESS \\
\hline
\end{tabular}




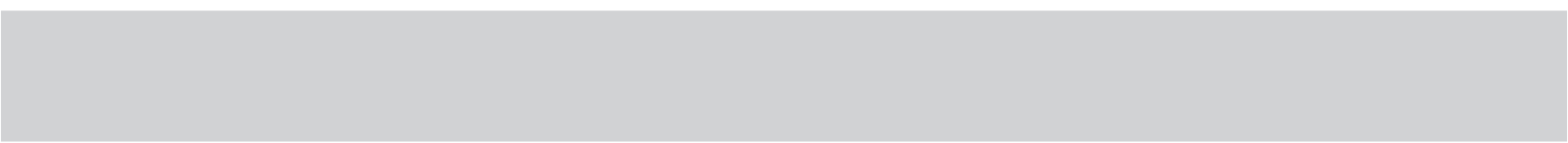

62 · ARL Statistics 2008-2009 


\section{Personnel and Public Services}

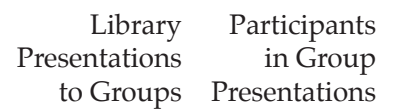

(30)

(31)
Reference
Transactions

(32)
Initial

Circulation

Transactions
Total
Circulation Transactions
Total Items

Loaned

(ILL)
Total Items

Borrowed

(ILL)
(35)
(33)
(34)
(36) (Survey Question Number)

INSTITUTION

\begin{tabular}{rrrrrrrr}
\hline 188 & 32,505 & 13,361 & 3,157 & 4,396 & 11,767 & 3,320 & NATIONAL AGRICULTURAL LIBRARY \\
26 & 200 & 23,530 & 1,333 & 251,187 & 32,828 & 1,383 & LIBRARY \& ARCHIVES CANADA \\
70 & 1,089 & 90,340 & 146,207 & 146,207 & 228,439 & 247 & NATIONAL LIBRARY OF MEDICINE \\
758 & 9,988 & 414,043 & UA/NA & UA/NA & 9,155 & 3,000 & NEW YORK PUBLIC \\
61 & 1,657 & 42,426 & 31,548 & 36,371 & 50,632 & 752 & NEW YORK STATE \\
1,057 & 4,073 & 57,385 & 40,913 & 232,182 & 5,346 & 6,079 & SMITHSONIAN
\end{tabular}

Figures in italics are derived from a sampling method rather than an actual count.

\section{Summary Data}

\begin{tabular}{rrrrrrr} 
Library & Participants & \multicolumn{2}{c}{ Initial } & \multicolumn{2}{c}{ Total } & Total Items \\
Presentations & in Group \\
to Groups & Presentations & $\begin{array}{r}\text { Reference } \\
\text { Transactions }\end{array}$ & $\begin{array}{r}\text { Circulation } \\
\text { Transactions }\end{array}$ & $\begin{array}{r}\text { Circulation } \\
\text { Transactions Loaned (ILL) }\end{array}$ & $\begin{array}{r}\text { Total Items } \\
\text { (ILL) }\end{array}$ \\
$(30)$ & $(31)$ & $(32)$ & $(33)$ & $(34)$ & $(35)$ & (36) (Survey Question Number)
\end{tabular}

\begin{tabular}{|c|c|c|c|c|c|c|c|}
\hline & & & & & & & University Libraries \\
\hline 1,003 & 17,619 & 72,622 & 315,348 & 575,892 & 39,855 & 32,109 & Mean \\
\hline 877 & 15,384 & 61,258 & 244,382 & 423,657 & 35,424 & 28,258 & Median \\
\hline 3,007 & 56,172 & 328,421 & $1,442,098$ & $2,513,529$ & 178,255 & 97,626 & High \\
\hline 174 & 2,980 & 12,310 & 43,661 & 56,984 & 6,843 & 4,764 & Low \\
\hline 112,344 & $1,973,311$ & $7,915,841$ & $34,688,244$ & $65,075,780$ & $4,543,485$ & $3,660,400$ & Totals \\
\hline \multirow[t]{2}{*}{112} & 112 & 109 & 110 & 113 & 114 & 114 & Number of Libraries Reporting \\
\hline & & & & & & & Nonuniversity Libraries \\
\hline 188 & 4,073 & 73,863 & 59,987 & 146,207 & 31,325 & 2,192 & Median \\
\hline 17,772 & 419,479 & $2,355,055$ & $4,838,320$ & $4,152,941$ & 423,574 & 32,316 & Totals \\
\hline 9 & 9 & 8 & 8 & 7 & 9 & 8 & Number of Libraries Reporting \\
\hline 130,116 & $2,392,790$ & $10,270,896$ & $39,526,564$ & $69,228,721$ & $4,967,059$ & $3,692,716$ & GRAND TOTALS \\
\hline
\end{tabular}




\section{Analysis of Selected Variables of University Libraries, 2008-2009}

The percentages and ratios below are select indicators that describe the condition of ARL university libraries. The high and low figures indicate the range, while the mean and median indicate the central tendency of the distributions for ARL university libraries. Note that a distribution is normal when the mean and the median figures are identical. If the mean is larger than the median then the distribution is positively skewed, and if the opposite is true the distribution is negatively skewed.

Category

Professional Staff as a percent of Total Staff

Support Staff as a percent of Total Staff

Student Assistant Staff as a percent of Total Staff

Ratio of Professional to Support Staff (excluding Student Assistant Staff)

Ratio of Items Loaned to Items Borrowed

Serials Expenditures as a percent of Total Library Materials

Expenditures

Monograph Expenditures as a percent of Total Library Materials Expenditures

Total Library Materials Expenditures as a percent of Total Library Expenditures

Contract Binding as a percent of Total Library Expenditures

Salary \& Wages Expenditures as a percent of Total Library Expenditures

Other Operating Expenditures as a percent of Total Library Expenditures

Unit costs of Monographs (per volume)

Unit costs of Serials (per title)

Library Expenditures per Faculty

Library Expenditures per Full-Time Student

Library Expenditures per Full-Time Graduate Student

Library Expenditures per Full-Time User

Library Expenditures per User

Library Expenditures per PhD Awarded

Library Staff per PhD Awarded

Professional Library Staff per PhD Awarded
High

59.72

71.30

53.05

3.31

5.18

91.83

40.54

64.39

2.04

59.01

23.46

125.87

443.38

$56,699.00$

$7,872.43$

$19,239.39$

$6,525.51$

$6,455.89$

$349,926.38$

5.32

1.70
Mean

Median

32.45

45.94

20.91

0.70

1.18

1.38

69.58

19.78

43.54

0.48

45.07

10.91

56.51

161.95

$17,907.26$

$1,349.38$

$5,525.60$

$1,236.12$

$1,078.40$

$91,501.96$

0.98

0.33
43.69

0.44

45.24

10.59

54.70

141.90

$15,562.90$

981.81

$4,787.41$

921.35

766.17

$79,932.78$

0.84

0.28
Low Number of Libraries

Reporting

20.12

114

14.97

0.46

114

0.38

0.23

14.50

113

113

114

114

114

114

106

108

114

114 


\section{PhD, Faculty, and Enrollment Statistics}

Enrollments

$\begin{array}{lllll}\text { PhDs Awarded PhD Fields Faculty } & \text { Total FTE Full-Time* } & \text { Total Part-Time* } & \begin{array}{c}\text { Graduate FTE } \\ \text { Full-Time }\end{array} & \begin{array}{c}\text { Graduate } \\ \text { Part-Time }\end{array}\end{array}$

(Survey Question Number)

(37)

(38)

(39)

(40)

(41)

(42)

(43)

INSTITUTION

\begin{tabular}{|c|c|c|c|c|c|c|c|}
\hline ALABAMA & 162 & 44 & 965 & 23,544 & 3,470 & 2,967 & 1,706 \\
\hline ALBERTA & 369 & 213 & 1,629 & 33,304 & 3,532 & 5,203 & 1,485 \\
\hline ARIZONA & 451 & 95 & 1,595 & 33,105 & 5,660 & 6,473 & 2,416 \\
\hline ARIZONA STATE & 470 & 63 & 2,549 & 51,614 & 15,468 & 8,469 & 5,315 \\
\hline AUBURN & 213 & 54 & 1,176 & 20,589 & 3,885 & 2,199 & 1,936 \\
\hline BOSTON & 290 & 60 & 2,814 & 25,629 & 6,137 & 8,616 & 4,616 \\
\hline BOSTON COLLEGE & 136 & 36 & 679 & 11,918 & 2,705 & 2,540 & 2,303 \\
\hline BRIGHAM YOUNG & 74 & 24 & 1,292 & 29,502 & 4,302 & 1,516 & 1,376 \\
\hline BRITISH COLUMBIA & 414 & 91 & 2,504 & 35,224 & 15,482 & 7,521 & 1,484 \\
\hline BROWN & 187 & 43 & 724 & 7,952 & 366 & 2,082 & 141 \\
\hline CALGARY & 300 & 43 & 1,720 & 24,470 & 3,445 & 4,222 & 1,154 \\
\hline CALIFORNIA, BERKELEY & 864 & 89 & 1,481 & 32,563 & 1,760 & 8,178 & 994 \\
\hline CALIFORNIA, DAVIS & 500 & 69 & 3,151 & 30,832 & 496 & 7,586 & 165 \\
\hline CALIFORNIA, IRVINE & 370 & 44 & 1,521 & 26,520 & 1,111 & 5,028 & 481 \\
\hline CALIFORNIA, LOS ANGELES & 752 & 83 & 2,654 & 38,332 & 1,320 & 10,741 & 397 \\
\hline CALIFORNIA, RIVERSIDE & 188 & 42 & 826 & 17,602 & 477 & 2,277 & 94 \\
\hline CALIFORNIA, SAN DIEGO & 437 & 67 & 943 & 23,143 & 934 & 4,231 & 101 \\
\hline $\begin{array}{l}\text { CALIFORNIA, SANTA } \\
\text { BARBARA }\end{array}$ & 347 & 46 & 908 & 21,392 & 476 & 2,958 & 18 \\
\hline CASE WESTERN RESERVE & 200 & 50 & 602 & 8,449 & 1,365 & 4,238 & 1,220 \\
\hline CHICAGO & 366 & 69 & 1,704 & 11,933 & 2,855 & 6,975 & 2,782 \\
\hline CINCINNATI & 353 & 58 & 1,235 & 20,914 & 7,124 & 5,159 & 3,544 \\
\hline COLORADO & 284 & 46 & 1,368 & 28,868 & 5,601 & 2,343 & 3,229 \\
\hline COLORADO STATE & 227 & 40 & 1,184 & 21,693 & 6,662 & 2,128 & 4,290 \\
\hline COLUMBIA & 518 & 106 & 2,166 & 24,864 & 5,731 & 15,966 & 4,775 \\
\hline CONNECTICUT & 266 & 70 & 1,817 & 20,456 & 3,817 & 4,373 & 3,135 \\
\hline CORNELL & 513 & 83 & 1,728 & 20,095 & 89 & 6,347 & 80 \\
\hline DARTMOUTH & 71 & 15 & 797 & 5,698 & 150 & 1,597 & 104 \\
\hline DELAWARE & 224 & 39 & 1,117 & 17,881 & 2,619 & 2,584 & 864 \\
\hline DUKE & 378 & 46 & 1,033 & 13,457 & 378 & 7,117 & 366 \\
\hline EMORY & 220 & 46 & 1,957 & 11,900 & 855 & 5,094 & 771 \\
\hline FLORIDA & 841 & 96 & 2,012 & 43,948 & 6,743 & 13,984 & 4,419 \\
\hline FLORIDA STATE & 345 & 76 & 1,425 & 33,261 & 6,625 & 6,465 & 3,134 \\
\hline GEORGE WASHINGTON & 136 & 27 & 1,192 & 15,798 & 9,318 & 7,746 & 8,783 \\
\hline GEORGETOWN & 103 & 36 & 845 & 13,072 & 2,246 & 6,266 & 1,960 \\
\hline GEORGIA & 918 & 162 & 3,502 & 59,774 & 8,586 & 8,960 & 5,360 \\
\hline GEORGIA TECH & 490 & 48 & 887 & 17,288 & 2,125 & 5,284 & 1,156 \\
\hline GUELPH & 106 & UA/NA & 869 & 17,521 & 2,009 & 2,000 & 201 \\
\hline HARVARD & UA/NA & UA/NA & 2,169 & 19,490 & 7,006 & 12,294 & 4,046 \\
\hline HAWAII & 183 & 51 & 1,343 & 13,861 & 2,225 & 3,268 & 1,018 \\
\hline
\end{tabular}




\section{PhD, Faculty, and Enrollment Statistics}

Enrollments

$\begin{array}{lllll}\text { PhDs Awarded PhD Fields Faculty } & \text { Total FTE Full-Time* } & \text { Total Part-Time* } & \begin{array}{c}\text { Graduate FTE } \\ \text { Full-Time }\end{array} & \begin{array}{c}\text { Graduate } \\ \text { Part-Time }\end{array}\end{array}$

(Survey Question Number)

(37)

(38)

(39)

(40)

(41)

(42)

(43)

INSTITUTION

\begin{tabular}{|c|c|c|c|c|c|c|c|}
\hline HOUSTON & 259 & 54 & 1,229 & 26,385 & 10,615 & 4,920 & 2,384 \\
\hline HOWARD & 96 & 29 & 1,048 & 8,638 & 2,589 & 2,667 & 1,079 \\
\hline ILLINOIS, CHICAGO & 580 & 114 & 1,201 & 19,048 & 4,489 & 4,562 & 3,310 \\
\hline ILLINOIS, URBANA & 782 & 95 & 2,358 & 41,004 & 1,126 & 10,345 & 612 \\
\hline INDIANA & 414 & 84 & 2,007 & 35,742 & 4,612 & 4,779 & 3,035 \\
\hline IOWA & 343 & 65 & 1,337 & 23,581 & 5,571 & 4,859 & 3,470 \\
\hline IOWA STATE & 316 & 80 & 1,411 & 24,738 & 3,207 & 2,781 & 2,079 \\
\hline JOHNS HOPKINS & 435 & 62 & 1,478 & 12,761 & 8,058 & 6,948 & 7,858 \\
\hline KANSAS & 308 & 76 & 1,768 & 24,583 & 4,782 & 5,351 & 2,682 \\
\hline KENT STATE & 150 & 50 & 903 & 18,388 & 4,556 & 2,390 & 2,402 \\
\hline KENTUCKY & 239 & 65 & 1,265 & 22,876 & 3,178 & 5,552 & 1,560 \\
\hline LAVAL & 290 & 54 & 1,454 & 25,381 & 10,995 & 5,316 & 4,135 \\
\hline LOUISIANA STATE & 234 & 55 & 1,413 & 26,395 & 2,723 & 4,214 & 1,255 \\
\hline LOUISVILLE & 494 & 36 & 1,552 & 21,761 & 5,734 & 6,266 & $\mathrm{UA} / \mathrm{NA}$ \\
\hline MCGILL & 455 & 7 & 3,257 & 27,515 & 6,693 & 7,334 & 2,849 \\
\hline MCMASTER & 169 & 47 & 1,253 & 22,340 & 3,631 & 2,809 & 603 \\
\hline MANITOBA & $\mathrm{UA} / \mathrm{NA}$ & 47 & 1,432 & 19,722 & 5,093 & 2,422 & 816 \\
\hline MARYLAND & 587 & 70 & 2,967 & 37,195 & 5,522 & 7,062 & 3,597 \\
\hline MASSACHUSETTS & 219 & 50 & 1,180 & 21,055 & 5,304 & 2,059 & 3,761 \\
\hline MIT & 607 & 36 & 961 & 10,109 & 190 & 5,991 & 155 \\
\hline MIAMI & 142 & 43 & 947 & 14,088 & 1,235 & 4,394 & 507 \\
\hline MICHIGAN & 816 & 117 & 3,839 & 38,278 & 2,750 & 13,100 & 1,924 \\
\hline MICHIGAN STATE & 498 & 112 & 2,010 & 42,044 & 5,572 & 8,558 & 2,721 \\
\hline MINNESOTA & 659 & 100 & 1,933 & 36,429 & 14,711 & 9,389 & 9,194 \\
\hline MISSOURI & 326 & 65 & 1,360 & 24,851 & 4,683 & 2,783 & 3,245 \\
\hline MONTREAL & 416 & 87 & 1,903 & 39,273 & 18,661 & 10,932 & 4,052 \\
\hline NEBRASKA & 238 & 37 & 1,249 & 20,495 & 3,489 & 3,212 & 2,246 \\
\hline NEW MEXICO & 166 & 42 & 1,846 & 18,838 & 7,629 & 2,637 & 2,241 \\
\hline NEW YORK & 423 & 72 & 3,695 & 32,237 & 9,952 & 12,395 & 8,525 \\
\hline NORTH CAROLINA & 440 & 67 & 1,600 & 23,788 & 4,779 & 6,738 & 3,934 \\
\hline NORTH CAROLINA STATE & 457 & 62 & 1,760 & 25,940 & 6,932 & 4,433 & 3,698 \\
\hline NORTHWESTERN & 449 & 96 & 3,070 & 16,079 & 2,492 & 7,742 & 2,127 \\
\hline NOTRE DAME & 165 & 22 & 907 & 11,602 & 129 & 3,256 & 112 \\
\hline OHIO & 166 & 57 & 1,111 & 24,317 & 5,396 & 2,934 & 865 \\
\hline OHIO STATE & $\mathrm{UA} / \mathrm{NA}$ & 103 & 3,046 & 52,419 & 9,149 & 9,447 & 4,370 \\
\hline OKLAHOMA & 210 & 58 & 2,151 & 23,303 & 6,560 & 5,476 & 3,640 \\
\hline OKLAHOMA STATE & 153 & 42 & 1,165 & 21,608 & 10,581 & 1,553 & 2,940 \\
\hline OREGON & 129 & 42 & 844 & 17,866 & 3,641 & 2,967 & 866 \\
\hline PENNSYLVANIA & 487 & 75 & 1,413 & 20,128 & 3,979 & 9,853 & 2,403 \\
\hline
\end{tabular}




\section{PhD, Faculty, and Enrollment Statistics}

Enrollments

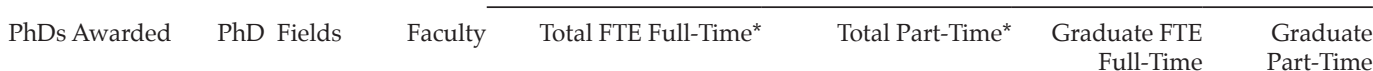

(Survey Question Number)

$(37)$

(38)

(39)

$(40)$

(41)

$(42)$

$(43)$

INSTITUTION

\begin{tabular}{|c|c|c|c|c|c|c|c|}
\hline PENNSYLVANIA STATE & 679 & 118 & 5,625 & 71,167 & 9,585 & 6,937 & 3,372 \\
\hline PITTSBURGH & 463 & 93 & 2,700 & 29,496 & 4,989 & 7,174 & 2,961 \\
\hline PRINCETON & 349 & 57 & 848 & 7,371 & 0 & 2,476 & 0 \\
\hline PURDUE & 652 & 58 & 2,110 & 35,873 & 4,658 & 5,173 & 2,253 \\
\hline QUEEN'S & 153 & 37 & 834 & 18,056 & 3,661 & 3,263 & 343 \\
\hline RICE & 168 & 30 & 628 & 5,224 & 133 & 2,139 & 98 \\
\hline ROCHESTER & 209 & 44 & 1,961 & 8,131 & 1,581 & 3,065 & 1,292 \\
\hline RUTGERS & 410 & 63 & 2,229 & 41,600 & 10,871 & 6,585 & 6,984 \\
\hline SASKATCHEWAN & 108 & 53 & 1,026 & 14,892 & 4,253 & 2,336 & 321 \\
\hline SOUTH CAROLINA & 230 & 61 & 1,419 & 24,352 & 4,374 & 4,287 & 2,882 \\
\hline SOUTHERN CALIFORNIA & 442 & 83 & 1,666 & 29,517 & 4,230 & 13,589 & 3,550 \\
\hline SOUTHERN ILLINOIS & 137 & 36 & 931 & 16,930 & 3,743 & 2,616 & 2,077 \\
\hline SUNY-ALBANY & 131 & 38 & 652 & 14,800 & 3,402 & 2,411 & 2,545 \\
\hline SUNY-BUFFALO & 373 & 78 & 1,237 & 23,808 & 4,384 & 6,028 & 3,142 \\
\hline SUNY-STONY BROOK & 206 & 38 & 925 & 19,292 & 4,702 & 4,557 & 3,513 \\
\hline SYRACUSE & 146 & 46 & 955 & 16,822 & 2,544 & 3,841 & 1,874 \\
\hline TEMPLE & 411 & 56 & 1,826 & 29,382 & 6,424 & 5,880 & 3,415 \\
\hline TENNESSEE & 410 & 78 & 1,580 & 26,795 & 3,615 & 6,467 & 2,226 \\
\hline TEXAS & 705 & 82 & 2,687 & 45,579 & 4,405 & 10,975 & 1,620 \\
\hline TEXAS A\&M & 594 & 96 & 2,803 & 44,568 & 5,445 & 7,532 & 2,123 \\
\hline TEXAS TECH & 179 & 57 & 1,797 & 26,941 & 4,097 & 5,166 & 2,157 \\
\hline TORONTO & 736 & 98 & 2,693 & 63,080 & 8,011 & 12,026 & 1,865 \\
\hline TULANE & 106 & 36 & 1,003 & 8,048 & 274 & 4,337 & 809 \\
\hline UTAH & 313 & 62 & 1,276 & 19,578 & 9,633 & 5,074 & 1,611 \\
\hline VANDERBILT & 260 & 48 & 2,721 & 11,318 & 775 & 4,735 & 721 \\
\hline VIRGINIA & 329 & 46 & 1,186 & 20,701 & 3,840 & 6,274 & 3,059 \\
\hline VIRGINIA TECH & 345 & 53 & 1,364 & 27,887 & 2,983 & 4,783 & 2,529 \\
\hline WASHINGTON & 678 & 82 & 3,728 & 38,059 & 8,716 & 9,863 & 3,118 \\
\hline WASHINGTON STATE & 195 & 44 & 1,204 & 20,958 & 4,394 & 2,089 & 1,307 \\
\hline WASHINGTON U.-ST. LOUIS & 247 & 58 & 1,861 & 11,158 & 2,181 & 5,121 & 1,233 \\
\hline WATERLOO & 214 & 48 & 951 & 26,457 & 2,388 & 3,315 & 677 \\
\hline WAYNE STATE & 210 & 48 & 1,021 & 18,064 & 12,960 & 5,736 & 5,166 \\
\hline WESTERN ONTARIO & 207 & 49 & 1,374 & 25,042 & 3,227 & 4,220 & 529 \\
\hline WISCONSIN & 778 & 109 & 2,017 & 37,308 & 4,722 & 9,265 & 2,015 \\
\hline YALE & 376 & 62 & 2,332 & 11,298 & 147 & 6,044 & 124 \\
\hline YORK & 144 & 25 & 1,528 & 42,775 & 9,214 & 3,902 & 2,008 \\
\hline
\end{tabular}

Figures were reported on the ARL Questionnaire and have not been verified with the US NCES IPEDS survey.

*-Includes both undergraduate and graduate students.

UA/NA- Unavailable or Not Applicable 
Summary Data

\begin{tabular}{|c|c|c|c|c|c|c|c|}
\hline & \multirow[b]{2}{*}{ PhDs Awarded } & \multirow[b]{2}{*}{ PhD Fields } & \multirow[b]{2}{*}{ Faculty } & \multicolumn{4}{|c|}{ ENROLLMENTS } \\
\hline & & & & Total FTE Full-time ** & Total Part-time** & $\begin{array}{r}\text { Graduate FTE } \\
\text { Full-time }\end{array}$ & $\begin{array}{l}\text { Graduate } \\
\text { Part-time }\end{array}$ \\
\hline (Survey Question Number) & (37) & (38) & (39) & $(40)$ & (41) & (42) & $(43)$ \\
\hline Median & 316 & 57 & 1,422 & 23,223 & 4,242 & 5,108 & 2,123 \\
\hline High & 918 & 213 & 5,625 & 71,167 & 18,661 & 15,966 & 9,194 \\
\hline Low & 71 & 7 & 602 & 5,224 & 0 & 1,516 & 0 \\
\hline Totals & 38,876 & 7,015 & 190,104 & $2,832,583$ & 540,704 & 642,702 & 264,190 \\
\hline Number of Libraries Reporting & 111 & 112 & 114 & 114 & 114 & 114 & 113 \\
\hline
\end{tabular}


RANK ORder Tables OF UNIVERSITy Libraries

2008-2009 


\section{Summary of RANK ORder TABLES For University LibraRies, 2008-2009}

The table below presents the rank for each university library in each of the 22 categories for which rank order tables are prepared. The table numbers in the chart below refer to the data categories listed below. The number of libraries indicates the number of ARL university libraries supplying data in each category.

\begin{tabular}{|c|c|c|}
\hline Table & Data Category & Number of Libraries Reporting \\
\hline 1 & VOLUMES IN LIBRARY & 114 \\
\hline 2 & VOLUMES ADDED (GROSS) & 114 \\
\hline 3 & TITLES HELD & 93 \\
\hline 4 & CURRENT SERIALS (TOTAL) & 114 \\
\hline 5 & MICROFORM UNITS & 111 \\
\hline 6 & GOVERNMENT DOCUMENTS & 100 \\
\hline 7 & TOTAL LIBRARY MATERIALS EXPENDITURES & 114 \\
\hline 8 & TOTAL SALARIES \& WAGES EXPENDITURES & 114 \\
\hline 9 & OTHER OPERATING EXPENDITURES & 114 \\
\hline 10 & TOTAL LIBRARY EXPENDITURES & 114 \\
\hline 11 & MONOGRAPHS PURCHASED (VOLUMES) & 107 \\
\hline 12 & EXPENDITURES FOR MONOGRAPHS & 113 \\
\hline 13 & CURRENT SERIALS PURCHASED (TITLES) & 109 \\
\hline 14 & EXPENDITURES FOR CURRENT SERIALS & 113 \\
\hline 15 & TOTAL ITEMS LOANED (ILL) & 114 \\
\hline 16 & TOTAL ITEMS BORROWED (ILL) & 114 \\
\hline 17 & PROFESSIONAL STAFF (FTE) & 114 \\
\hline 18 & SUPPORT STAFF (FTE) & 114 \\
\hline 19 & TOTAL STAFF (FTE) & 114 \\
\hline 20 & EXPENDITURES FOR ELECTRONIC RESOURCES & 113 \\
\hline 21 & ELECTRONIC RESOURCES AS A \% OF TOTAL LIBRARY MATERIALS & 113 \\
\hline 22 & LIBRARY INVESTMENT INDEX & 114 \\
\hline
\end{tabular}




\begin{tabular}{|c|c|c|c|c|c|c|c|c|c|c|c|c|c|c|c|c|c|c|c|c|c|c|}
\hline Table Number & 1 & 2 & 3 & 4 & 5 & 6 & 7 & 8 & 9 & 10 & 11 & 12 & 13 & 14 & 15 & 16 & 17 & 18 & 19 & 20 & 21 & 22 \\
\hline ALABAMA & 69 & 41 & 40 & 43 & 63 & 13 & 85 & 95 & 65 & 90 & 13 & 91 & 59 & 69 & 112 & 103 & 67 & 95 & 78 & 62 & 12 & 89 \\
\hline ALBERTA & 16 & 8 & 26 & 5 & 73 & 67 & 16 & 24 & 3 & 15 & UA & 9 & 3 & 20 & 30 & 89 & 51 & 14 & 35 & 1 & 1 & 16 \\
\hline ARIZONA & 24 & 16 & 9 & 37 & 24 & 67 & 34 & 43 & 13 & 30 & 55 & 42 & 11 & 26 & 22 & 20 & 46 & 48 & 40 & 16 & 19 & 32 \\
\hline ARIZONA STATE & 34 & 83 & UA & 23 & 10 & 21 & 51 & 46 & 70 & 53 & 61 & 85 & 14 & 27 & 26 & 22 & 46 & 26 & 32 & 20 & 7 & 54 \\
\hline AUBURN & 66 & 110 & 28 & 97 & 92 & 44 & 108 & 112 & 91 & 113 & 102 & 110 & 104 & 99 & 106 & 106 & 108 & 111 & 110 & 82 & 2 & 112 \\
\hline BOSTON & 89 & 101 & 43 & 85 & 45 & UA & 73 & 68 & 64 & 66 & 86 & 99 & 65 & 38 & 90 & 78 & 34 & 72 & 44 & 78 & 74 & 66 \\
\hline BOSTON COLLEGE & 99 & 94 & 70 & 108 & 58 & 46 & 74 & 71 & 89 & 78 & 70 & 72 & 101 & 97 & 73 & 84 & 65 & 98 & 86 & 70 & 56 & 75 \\
\hline BRIGHAM YOUNG & 39 & 38 & 24 & 51 & 70 & 30 & 68 & 39 & 37 & 47 & 18 & 38 & 66 & 64 & 15 & 49 & 23 & 104 & 22 & 71 & 68 & 47 \\
\hline BRITISH COLUMBIA & 19 & 22 & 22 & 42 & 39 & 67 & 21 & 28 & 32 & 25 & 48 & 23 & 9 & 9 & 83 & 92 & 25 & 24 & 33 & 14 & 44 & 26 \\
\hline BROWN & 48 & 56 & 38 & 75 & 102 & 67 & 78 & 65 & 66 & 69 & 40 & 64 & 70 & 84 & 51 & 32 & 63 & 70 & 79 & 76 & 62 & 69 \\
\hline CALGARY & 73 & 31 & 67 & 98 & 74 & 67 & 43 & 41 & 100 & 49 & 41 & 27 & 84 & 51 & 92 & 61 & 71 & 29 & 52 & 40 & 57 & 49 \\
\hline CALIFORNIA, BERKELEY & 5 & 15 & UA & 7 & 12 & 27 & 10 & 5 & 38 & 6 & UA & 6 & 19 & 14 & 50 & 64 & 5 & 18 & 7 & 46 & 109 & 6 \\
\hline CALIFORNIA, DAVIS & 41 & 45 & 58 & 57 & 56 & 53 & 80 & 63 & 102 & 76 & 22 & 58 & 57 & 74 & 44 & 71 & 93 & 49 & 76 & 80 & 63 & 77 \\
\hline CALIFORNIA, IRVINE & 77 & 39 & 66 & 89 & 90 & 50 & 70 & 62 & 68 & 64 & 56 & 53 & 85 & 60 & 74 & 58 & 74 & 50 & 64 & 68 & 59 & 65 \\
\hline CALIFORNIA, LOS ANGELES & 9 & 33 & UA & 107 & 25 & 52 & 26 & 6 & 6 & 7 & 35 & 10 & 99 & 42 & 33 & 35 & 12 & 8 & 9 & 73 & 108 & 8 \\
\hline CALIFORNIA, RIVERSIDE & 85 & 92 & 52 & 30 & 98 & 38 & 112 & 88 & 97 & 107 & 100 & 108 & 37 & 107 & 54 & 66 & 108 & 89 & 105 & 110 & 92 & 107 \\
\hline CALIFORNIA, SAN DIEGO & 59 & 25 & UA & 36 & 81 & 67 & 63 & 25 & 40 & 33 & 53 & 29 & 40 & 68 & 65 & 60 & 32 & 21 & 27 & 60 & 54 & 35 \\
\hline CALIFORNIA, SANTA BARBARA & 82 & 76 & 33 & 70 & 72 & 29 & 110 & 48 & 55 & 81 & 85 & 93 & 86 & 108 & 85 & 38 & 106 & 52 & 75 & 106 & 42 & 86 \\
\hline CASE WESTERN RESERVE & 92 & 102 & 59 & 55 & 93 & 57 & 98 & 107 & 88 & 103 & 105 & 105 & 30 & 87 & 62 & 41 & 86 & 111 & 109 & 104 & 58 & 102 \\
\hline CHICAGO & 10 & 4 & 7 & 11 & 85 & UA & 8 & 34 & 33 & 24 & 9 & 7 & 44 & 7 & 23 & 86 & 72 & 22 & 37 & 38 & 103 & 24 \\
\hline CINCINNATI & 55 & 47 & 48 & 10 & 80 & 58 & 59 & 82 & 71 & 72 & 58 & 49 & 6 & 57 & 18 & 30 & 61 & 107 & 94 & 67 & 69 & 71 \\
\hline COLORADO & 35 & 52 & UA & 81 & 13 & 10 & 49 & 72 & 81 & 63 & 23 & 51 & 78 & 50 & 24 & 46 & 91 & 55 & 67 & 39 & 45 & 63 \\
\hline COLORADO STATE & 105 & 51 & 50 & 91 & 110 & UA & 99 & 102 & 54 & 97 & 19 & 92 & 92 & 100 & 7 & 7 & 104 & 107 & 110 & 83 & 8 & 98 \\
\hline COLUMBIA & 6 & 5 & 4 & 1 & 22 & 49 & 3 & 4 & 7 & 3 & 4 & 5 & 1 & 1 & 35 & 10 & 2 & 12 & 4 & 2 & 86 & 3 \\
\hline CONNECTICUT & 47 & 80 & 51 & 24 & 55 & 67 & 60 & 29 & 78 & 42 & 93 & 90 & 61 & 47 & 46 & 16 & 55 & 102 & 80 & 35 & 11 & 42 \\
\hline CORNELL & 13 & 32 & 12 & 12 & 6 & 67 & 20 & 9 & 8 & 10 & UA & 8 & UA & 35 & 21 & 44 & 29 & 7 & 11 & 30 & 90 & 12 \\
\hline DARTMOUTH & 90 & 64 & 56 & 77 & 95 & UA & 72 & 74 & 86 & 77 & 29 & 65 & 46 & 49 & 43 & 52 & 93 & 58 & 82 & 47 & 21 & 78 \\
\hline DELAWARE & 91 & 104 & 63 & 112 & 79 & 28 & 76 & 79 & 82 & 84 & 78 & 47 & 103 & 86 & 95 & 70 & 89 & 76 & 88 & 55 & 22 & 84 \\
\hline DUKE & 22 & 19 & UA & 25 & 54 & 5 & 18 & 21 & 30 & 22 & 15 & 16 & 51 & 15 & 57 & 55 & 8 & 43 & 30 & 19 & 81 & 21 \\
\hline EMORY & 63 & 42 & 64 & 39 & 27 & 43 & 15 & 32 & 27 & 26 & 36 & 22 & 32 & 70 & 53 & 87 & 45 & 33 & 42 & 52 & 107 & 25 \\
\hline FLORIDA & 36 & 86 & UA & 31 & 8 & 6 & 38 & 30 & 73 & 40 & 65 & 76 & 63 & 24 & 96 & 95 & 43 & 23 & 14 & 88 & 106 & 39 \\
\hline FLORIDA STATE & 80 & 98 & 42 & 49 & 4 & 14 & 88 & 90 & 114 & 95 & 88 & 97 & 52 & 83 & 84 & 99 & 79 & 71 & 65 & 59 & 9 & 94 \\
\hline GEORGE WASHINGTON & 110 & 60 & 90 & 56 & 108 & 63 & 52 & 47 & 29 & 44 & 66 & 41 & 33 & 59 & 64 & 9 & 55 & 61 & 58 & 72 & 91 & 44 \\
\hline GEORGETOWN & 67 & 59 & 31 & 64 & 44 & UA & 41 & 40 & 43 & 41 & 50 & 40 & 36 & 43 & 58 & 36 & 42 & 57 & 53 & 75 & 100 & 38 \\
\hline GEORGIA & 32 & 48 & 23 & 21 & 17 & 8 & 57 & 59 & 77 & 62 & 43 & 63 & 28 & 45 & 45 & 111 & 60 & 26 & 41 & 57 & 60 & 62 \\
\hline GEORGIA TECH & 100 & 113 & 93 & 103 & 48 & 67 & 105 & 109 & 101 & 110 & 104 & 112 & 109 & 103 & 109 & 101 & 107 & 99 & 110 & 84 & 3 & 110 \\
\hline GUELPH & 114 & 93 & 88 & 94 & 97 & 67 & 107 & 108 & 103 & 112 & 99 & 87 & 67 & 111 & 40 & 18 & 100 & 99 & 108 & 102 & 23 & 111 \\
\hline HARVARD & 1 & 1 & UA & 2 & 3 & UA & 2 & 1 & 1 & 1 & UA & 2 & UA & 36 & 47 & 96 & 1 & 1 & 1 & 8 & 111 & 1 \\
\hline HAWAII & 61 & 63 & 54 & 72 & 77 & 67 & 87 & 64 & 107 & 82 & 71 & 89 & 74 & 67 & 114 & 54 & 49 & 89 & 60 & 65 & 13 & 80 \\
\hline HOUSTON & 94 & 82 & 41 & 44 & 30 & 18 & 62 & 100 & 34 & 74 & 84 & 69 & 64 & 73 & 81 & 91 & 82 & 95 & 90 & 56 & 50 & 74 \\
\hline HOWARD & 96 & 112 & UA & 114 & 57 & 65 & 114 & 114 & 112 & 114 & 107 & 113 & 106 & 112 & 113 & 114 & 112 & 106 & 101 & 109 & 34 & 114 \\
\hline ILLINOIS, CHICAGO & 101 & 62 & 61 & 73 & 60 & 15 & 55 & 81 & 74 & 71 & 46 & 81 & 77 & 104 & 14 & 12 & 76 & 77 & 92 & 113 & 113 & 70 \\
\hline ILLINOIS, URBANA & 2 & 3 & 3 & 4 & 1 & 67 & 25 & 13 & 19 & 16 & 8 & 33 & 2 & 12 & 5 & 5 & 7 & 19 & 13 & 24 & 71 & 15 \\
\hline INDIANA & 11 & 18 & 6 & 35 & 43 & 37 & 23 & 27 & 52 & 29 & 14 & 11 & 20 & 37 & 37 & 17 & 20 & 30 & 18 & 31 & 79 & 28 \\
\hline IOWA & 30 & 12 & 14 & 60 & UA & UA & 28 & 33 & 84 & 35 & 32 & 39 & 55 & 11 & 19 & 45 & 34 & 53 & 43 & 29 & 72 & 33 \\
\hline
\end{tabular}


\begin{tabular}{lllllllllllllllllllllll} 
Table Number & 1 & 2 & 3 & 4 & 5 & 6 & 7 & 8 & 9 & 10 & 11 & 12 & 13 & 14 & 15 & 16 & 17 & 18 & 19 & 20 & 21 & 22 \\
\hline
\end{tabular}

\begin{tabular}{lllllllllllllllllllllllllllll}
\hline IOWASTATE & 97 & 91 & 85 & 18 & 76 & 67 & 37 & 86 & 45 & 59 & 68 & 67 & 31 & 16 & 61 & 88 & 97 & 84 & 98 & 44 & 77 & 59
\end{tabular}
$\begin{array}{llllllllllllllllllllllllllllll} & \\ \text { JOHNS HOPKINS } & 54 & 105 & \mathrm{UA} & 14 & 52 & 67 & 24 & 50 & 36 & 36 & 69 & 36 & 39 & 52 & 38 & 25 & 24 & 41 & 38 & 5 & 5 & 36\end{array}$ $\begin{array}{lllllllllllllllllllllllllll}\text { KANSAS } & 38 & 96 & 20 & 58 & 84 & 17 & 66 & 52 & 60 & 60 & 51 & 57 & 50 & 72 & 29 & 33 & 37 & 58 & 39 & 54 & 37 & 60\end{array}$ $\begin{array}{llllllllllllllllllllllll}\text { KENT STATE } & 88 & 99 & 83 & 48 & \text { UA } & 67 & 113 & 111 & 47 & 109 & 94 & 102 & 48 & 113 & 105 & 110 & 93 & 113 & 114 & 111 & 80 & 113\end{array}$ $\begin{array}{llllllllllllllllllllllllllllll}\text { KENTUCKY } & 50 & 66 & 53 & 50 & 19 & 9 & 54 & 75 & 63 & 65 & 59 & 74 & 72 & 65 & 67 & 65 & 52 & 53 & 50 & 48 & 55 & 64\end{array}$

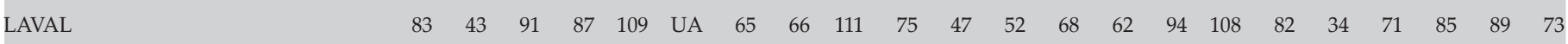
$\begin{array}{llllllllllllllllllllllllll}\text { LOUISIANA STATE } & 44 & 103 & 60 & 9 & 5 & 23 & 103 & 101 & 105 & 105 & 63 & 96 & 4 & 96 & 100 & 94 & 100 & 93 & 91 & 101 & 28 & 105\end{array}$ $\begin{array}{lllllllllllllllllllllll}\text { LOUISVILLE } & 112 & 65 & \text { UA } & 34 & 100 & \text { UA } & 71 & 99 & 35 & 79 & 54 & 100 & 7 & 106 & 80 & 56 & 100 & 77 & 93 & 100 & 98 & 79\end{array}$ $\begin{array}{lllllllllllllllllllllllllll}\text { MCGILL } & 42 & 34 & 36 & 76 & 103 & 19 & 33 & 49 & 25 & 38 & 3 & 18 & 62 & 39 & 88 & 98 & 63 & 35 & 55 & 28 & 52 & 40\end{array}$ $\begin{array}{lllllllllllllllllllllllll}\text { MCMASTER } & 113 & 9 & 81 & 68 & 106 & 67 & 90 & 91 & 62 & 89 & 30 & 84 & 80 & 90 & 102 & 97 & 90 & 89 & 97 & 99 & 78 & 91\end{array}$ $\begin{array}{lllllllllllllllllllllllllll}\text { MANITOBA } & 107 & 55 & 86 & 92 & 107 & 45 & 91 & 55 & 76 & 73 & 11 & 66 & 97 & 92 & 93 & 69 & 78 & 41 & 71 & 97 & 70 & 76\end{array}$ $\begin{array}{lllllllllllllllllllllllllll}\text { MARYLAND } & 51 & 74 & \mathrm{UA} & 93 & 31 & 47 & 69 & 36 & 41 & 48 & 12 & 70 & 102 & 93 & 77 & 34 & 26 & 72 & 50 & 50 & 24 & 48\end{array}$ $\begin{array}{llllllllllllllllllllllllllll}\text { MASSACHUSETTS } & 58 & 54 & \text { UA } & 90 & 94 & 67 & 101 & 85 & 92 & 96 & 101 & 101 & 81 & 98 & 48 & 23 & 86 & 97 & 88 & 91 & 17 & 97\end{array}$ MIT

MIAMI

$\begin{array}{llllllllllllllllllllll}79 & 79 & \text { UA } & 83 & 96 & 67 & 75 & 38 & 42 & 50 & 79 & 71 & 75 & 58 & 97 & 81 & 44 & 79 & 77 & 61 & 32 & 50\end{array}$ MICHIGAN

$\begin{array}{llllllllllllllllllllll}71 & 61 & 49 & 53 & 66 & 67 & 31 & 67 & 44 & 43 & 44 & 48 & 21 & 8 & 60 & 100 & 58 & 46 & 45 & 11 & 16 & 43\end{array}$ $\begin{array}{lllllllllllllllllllllllllll}\text { MINNESOTA } & 17 & 20 & 13 & 27 & 14 & 2 & 14 & 18 & 15 & 17 & 52 & 30 & 22 & 3 & 1 & 28 & 31 & 17 & 23 & 13 & 67 & 18\end{array}$ $\begin{array}{llllllllllllllllllllllllllllll}\text { MISSOURI } & 62 & 97 & 35 & 102 & 7 & 3 & 82 & 94 & 80 & 91 & 60 & 94 & 98 & 55 & 20 & 24 & 91 & 69 & 86 & 45 & 6 & 90\end{array}$ $\begin{array}{lllllllllllllllllllllllllll}\text { MONTREAL } & 75 & 73 & 76 & 28 & 105 & 67 & 39 & 26 & 104 & 34 & 26 & 34 & 8 & 23 & 71 & 83 & 30 & 9 & 26 & 34 & 51 & 34\end{array}$ $\begin{array}{lllllllllllllllllllllllllllll} & \text { NEBRASKA } & 72 & 88 & 62 & 105 & 47 & 24 & 100 & 89 & 90 & 98 & 103 & 107 & 88 & 88 & 63 & 53 & 96 & 66 & 81 & 92 & 20 & 100\end{array}$ $\begin{array}{llllllllllllllllllllllllllllll} & \text { NEW MEXICO } & 78 & 36 & 45 & 52 & 46 & 22 & 89 & 56 & 69 & 70 & 73 & 80 & 45 & 85 & 79 & 48 & 85 & 45 & 61 & 98 & 75 & 72\end{array}$ $\begin{array}{llllllllllllllllllllllll}\text { NEW YORK } & 29 & 26 & 30 & 6 & 33 & 26 & 9 & 14 & 9 & 12 & 33 & 13 & 16 & 19 & 78 & 31 & 16 & 11 & 15 & 9 & 66 & 11\end{array}$ $\begin{array}{lllllllllllllllllllllllllll}\text { NORTH CAROLINA } & 18 & 27 & 21 & 46 & 41 & 4 & 19 & 15 & 28 & 18 & 20 & 28 & 60 & 66 & 13 & 85 & 14 & 14 & 17 & 18 & 73 & 17\end{array}$ $\begin{array}{lllllllllllllllllllllllllllll}\text { NORTH CAROLINA STATE } & 40 & 57 & 71 & 79 & 35 & 67 & 64 & 42 & 10 & 37 & 81 & 75 & 89 & 54 & 91 & 62 & 21 & 64 & 48 & 94 & 96 & 41\end{array}$ $\begin{array}{lllllllllllllllllllllllllllll} & \text { NORTHWESTERN } & 31 & 44 & \text { UA } & 16 & 50 & 60 & 30 & 35 & 51 & 31 & 37 & 24 & 24 & 25 & 49 & 42 & 26 & 39 & 31 & 25 & 61 & 30\end{array}$ $\begin{array}{llllllllllllllllllllllllll}\text { NOTRE DAME } & 64 & 50 & 47 & 32 & 67 & \text { UA } & 45 & 53 & 61 & 51 & 39 & 15 & 29 & 56 & 76 & 68 & 59 & 37 & 49 & 63 & 85 & 51\end{array}$ $\begin{array}{llllllllllllllllllllllllll}\text { OHIO } & 84 & 46 & 69 & 101 & 101 & 67 & 109 & 105 & 79 & 108 & 67 & 98 & 93 & 110 & 4 & 6 & 104 & 101 & 100 & 108 & 65 & 108\end{array}$ $\begin{array}{llllllllllllllllllllllllllll}\text { OHIOSTATE } & 21 & 24 & 16 & 29 & 28 & 66 & 36 & 20 & 5 & 21 & 17 & 37 & 12 & 30 & 2 & 2 & 9 & 35 & 16 & 66 & 97 & 22\end{array}$ $\begin{array}{lllllllllllllllllllllllllllll}\text { OKLAHOMA } & 26 & 21 & 27 & 69 & 51 & 56 & 27 & 93 & 67 & 56 & 42 & 25 & 49 & 32 & 42 & 47 & 82 & 72 & 62 & 74 & 110 & 55\end{array}$ \begin{tabular}{llllllllllllllllllllllllllllll}
\hline OKLAHOMA STATE & 86 & 67 & 77 & 63 & 111 & 48 & 97 & 76 & 58 & 88 & 28 & UA & 53 & UA & 66 & 72 & 75 & 93 & 68 & UA & UA & 88
\end{tabular} $\begin{array}{lllllllllllllllllllllllllll}\text { OREGON } & 81 & 89 & 79 & 100 & 62 & 42 & 102 & 78 & 93 & 94 & 91 & 88 & 100 & 109 & 12 & 8 & 68 & 88 & 68 & 107 & 87 & 95\end{array}$ $\begin{array}{lllllllllllllllllllllllllllll}\text { PENNSYLVANIA } & 20 & 28 & \text { UA } & 59 & 61 & \text { UA } & 22 & 19 & 11 & 19 & \text { UA } & 19 & 34 & 17 & 10 & 4 & 19 & 25 & 20 & 32 & 83 & 20\end{array}$ $\begin{array}{lllllllllllllllllllllllllllll}\text { PENNSYLVANIA STATE } & 27 & 35 & 18 & 15 & 40 & 67 & 7 & 8 & 16 & 8 & 27 & 17 & 25 & 4 & 9 & 11 & 13 & 3 & 6 & 4 & 43 & 7\end{array}$ $\begin{array}{lllllllllllllllllllllllllll}\text { PITTSBURGH } & 23 & 7 & \text { UA } & 33 & 37 & 67 & 29 & 37 & 49 & 32 & \text { UA } & 32 & 13 & 22 & 8 & 59 & 26 & 26 & 29 & 12 & 25 & 31\end{array}$ $\begin{array}{llllllllllllllllllllllllllll}\text { PRINCETON } & 15 & 17 & 11 & 82 & 18 & 64 & 6 & 16 & 14 & 9 & 10 & 3 & 47 & 13 & 32 & 39 & 18 & 13 & 20 & 15 & 102 & 9\end{array}$ $\begin{array}{llllllllllllllllllllllllllll}\text { PURDUE } & 102 & 107 & 73 & 99 & 87 & 25 & 50 & 51 & 53 & 52 & 96 & 78 & 87 & 46 & 70 & 21 & 77 & 66 & 66 & 33 & 26 & 52\end{array}$ $\begin{array}{llllllllllllllllllllllllll}\text { QUEEN'S } & 98 & 100 & \text { UA } & 67 & 65 & 7 & 83 & 87 & 98 & 92 & 74 & 86 & 58 & 75 & 98 & 112 & 111 & 61 & 95 & 77 & 48 & 93\end{array}$ $\begin{array}{llllllllllllllllllllllll}\text { RICE } & 95 & 87 & 65 & 45 & 82 & 55 & 56 & 113 & 106 & 93 & 45 & 26 & 38 & 77 & 107 & 105 & 86 & 109 & 113 & 90 & 99 & 92\end{array}$ $\begin{array}{lllllllllllllllllllllllllll}\text { ROCHESTER } & 53 & 108 & 34 & 109 & 42 & 31 & 84 & 80 & 57 & 83 & 83 & 83 & 94 & 63 & 86 & 67 & 40 & 104 & 73 & 105 & 94 & 81\end{array}$ $\begin{array}{llllllllllllllllllllllllllll}\text { RUTGERS } & 33 & 58 & 74 & 71 & 29 & 1 & 48 & 17 & 72 & 28 & 82 & 61 & 73 & 34 & 39 & 40 & 49 & 10 & 19 & 37 & 40 & 29\end{array}$ $\begin{array}{lllllllllllllllllllllllllllll} & \text { SASKATCHEWAN } & 106 & 95 & 84 & 104 & 88 & 32 & 67 & 92 & 87 & 87 & 89 & 54 & 95 & 61 & 101 & 82 & 100 & 83 & 102 & 93 & 95 & 85\end{array}$ $\begin{array}{lllllllllllllllllllllllllllllllllll} & \text { SOUTH CAROLINA } & 56 & 84 & 25 & 74 & 38 & 11 & 86 & 84 & 39 & 80 & 95 & 73 & 107 & 78 & 99 & 90 & 68 & 65 & 46 & 81 & 46 & 83\end{array}$

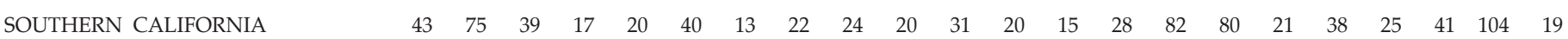

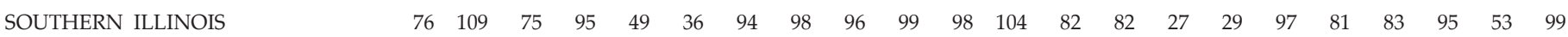




\begin{tabular}{|c|c|c|c|c|c|c|c|c|c|c|c|c|c|c|c|c|c|c|c|c|c|c|}
\hline Table Number & 1 & 2 & 3 & 4 & 5 & 6 & 7 & 8 & 9 & 10 & 11 & 12 & 13 & 14 & 15 & 16 & 17 & 18 & 19 & 20 & 21 & 22 \\
\hline SUNY-ALBANY & 111 & 90 & 89 & 96 & 89 & 67 & 111 & 97 & 113 & 111 & 92 & 109 & 83 & 105 & 110 & 75 & 68 & 110 & 104 & 112 & 105 & 109 \\
\hline SUNY-BUFFALO & 49 & 11 & 44 & 47 & 26 & 67 & 77 & 57 & 85 & 68 & 76 & 68 & 41 & 71 & 72 & 73 & 39 & 103 & 68 & 53 & 18 & 67 \\
\hline SUNY-STONY BROOK & 109 & 114 & 92 & 22 & 71 & 35 & 96 & 104 & 108 & 104 & 106 & 111 & 10 & 81 & 87 & 102 & 54 & 114 & 107 & 89 & 31 & 101 \\
\hline SYRACUSE & 74 & 72 & 80 & 106 & 11 & 39 & 92 & 73 & 75 & 86 & 80 & 79 & 96 & 91 & 111 & 77 & 79 & 75 & 83 & 86 & 47 & 87 \\
\hline TEMPLE & 52 & 69 & 68 & 80 & 83 & 59 & 42 & 77 & 59 & 61 & 49 & 45 & 69 & 80 & 69 & 63 & 65 & 79 & 74 & 26 & 27 & 58 \\
\hline TENNESSEE & 70 & 70 & 57 & 84 & 53 & 62 & 40 & 69 & 83 & 58 & 38 & 44 & 79 & 29 & 59 & 76 & 52 & 47 & 56 & 27 & 33 & 56 \\
\hline TEXAS & 7 & 30 & 10 & 19 & 16 & 67 & 12 & 12 & 12 & 11 & 25 & 35 & 26 & 18 & 16 & 57 & 15 & 6 & 10 & 21 & 88 & 10 \\
\hline TEXAS A\&M & 45 & 14 & 29 & 26 & 32 & 54 & 11 & 31 & 17 & 23 & 6 & 14 & 42 & 5 & 36 & 13 & 17 & 43 & 28 & 6 & 49 & 23 \\
\hline TEXAS TECH & 93 & 81 & UA & 38 & 91 & 61 & 47 & 60 & 31 & 46 & 57 & 46 & 43 & 41 & 56 & 51 & 37 & 40 & 34 & 58 & 82 & 46 \\
\hline TORONTO & 4 & 6 & 5 & 41 & 34 & UA & 4 & 3 & 18 & 4 & 5 & 4 & 18 & 10 & 68 & 109 & 10 & 4 & 3 & 3 & 84 & 4 \\
\hline TULANE & 46 & 37 & UA & 40 & 99 & 51 & 79 & 110 & 110 & 100 & 1 & 59 & 56 & 76 & 104 & 104 & 108 & 85 & 103 & 64 & 30 & 96 \\
\hline UTAH & 68 & 77 & UA & 61 & 75 & 12 & 93 & 45 & 21 & 55 & UA & 62 & 90 & 94 & 41 & 37 & 57 & 32 & 36 & 87 & 39 & 61 \\
\hline VANDERBILT & 65 & 85 & 37 & 88 & 86 & 41 & 53 & 61 & 46 & 54 & 77 & 77 & 91 & 21 & 75 & 93 & 33 & 66 & 59 & 23 & 10 & 53 \\
\hline VIRGINIA & 25 & 23 & 19 & 3 & 36 & 20 & 32 & 23 & 26 & 27 & 21 & 31 & UA & 48 & 52 & 50 & 34 & 16 & 24 & 69 & 101 & 27 \\
\hline VIRGINIA TECH & 104 & 71 & 87 & 113 & 23 & 33 & 95 & 106 & 99 & 102 & 87 & 82 & 108 & 95 & 89 & 79 & 112 & 86 & 106 & 79 & 15 & 104 \\
\hline WASHINGTON & 14 & 13 & 15 & 78 & 9 & UA & 17 & 11 & 23 & 13 & 24 & 21 & 76 & 6 & 3 & 1 & 6 & 20 & 12 & 17 & 76 & 13 \\
\hline WASHINGTON STATE & 103 & 78 & 72 & 110 & 68 & 67 & 104 & 96 & 94 & 101 & 97 & 95 & UA & 101 & 55 & 74 & 97 & 86 & 95 & 96 & 14 & 103 \\
\hline WASHINGTON U.-ST. LOUIS & 37 & 68 & 32 & 62 & 78 & 67 & 35 & 58 & 20 & 39 & 64 & 55 & 54 & 33 & 17 & 15 & 46 & 55 & 54 & 22 & 35 & 37 \\
\hline WATERLOO & 108 & 106 & 82 & 111 & 104 & 67 & 106 & 103 & 95 & 106 & 62 & 103 & 105 & 102 & 28 & 19 & 112 & 81 & 99 & 103 & 29 & 106 \\
\hline WAYNE STATE & 57 & 111 & 55 & 86 & 69 & 34 & 81 & 70 & 48 & 67 & 90 & 106 & 71 & 44 & 31 & 26 & 40 & 89 & 57 & 42 & 4 & 68 \\
\hline WESTERN ONTARIO & 60 & 40 & 46 & 66 & 64 & 67 & 58 & 83 & 109 & 85 & 34 & 60 & 35 & 40 & 108 & 107 & 72 & 63 & 83 & 49 & 38 & 82 \\
\hline WISCONSIN & 12 & 29 & 8 & 13 & 21 & 16 & 46 & 10 & 4 & 14 & 16 & 56 & 23 & 79 & 6 & 3 & 4 & 30 & 5 & 43 & 64 & 14 \\
\hline YALE & 3 & 2 & 2 & 20 & 2 & 67 & 1 & 2 & 2 & 2 & 2 & 1 & 5 & 89 & 34 & 27 & 3 & 2 & 2 & 10 & 112 & 2 \\
\hline YORK & 87 & 49 & 78 & 54 & 59 & UA & 61 & 54 & 50 & 57 & 72 & 50 & 27 & 53 & 103 & 113 & 79 & 60 & 63 & 51 & 36 & 57 \\
\hline
\end{tabular}

UA - Unavailable 
Rank Order Table 1: Volumes in Library

\begin{tabular}{|c|c|c|c|c|c|}
\hline RANK & INSTITUTION & Value & RANK & INSTITUTION & VALUE \\
\hline 1 & HARVARD & $16,557,002$ & 58 & MASSACHUSETTS & $3,654,181$ \\
\hline 2 & ILLINOIS, URBANA & $12,780,067$ & 59 & CALIFORNIA, SAN DIEGO & $3,651,393$ \\
\hline 3 & YALE & $12,564,157$ & 60 & WESTERN ONTARIO & $3,644,679$ \\
\hline 4 & TORONTO & $11,345,102$ & 61 & HAWAII & $3,588,005$ \\
\hline 5 & CALIFORNIA, BERKELEY & $11,026,554$ & 62 & MISSOURI & $3,523,795$ \\
\hline 6 & COLUMBIA & $10,449,223$ & 63 & EMORY & $3,479,536$ \\
\hline 7 & TEXAS & $9,853,414$ & 64 & NOTRE DAME & $3,469,001$ \\
\hline 8 & MICHIGAN & $9,575,256$ & 65 & VANDERBILT & $3,467,542$ \\
\hline 9 & CALIFORNIA, LOS ANGELES & $9,045,818$ & 66 & AUBURN & $3,459,542$ \\
\hline 10 & CHICAGO & $8,830,151$ & 67 & GEORGETOWN & $3,431,948$ \\
\hline 11 & INDIANA & $8,543,025$ & 68 & UTAH & $3,418,976$ \\
\hline 12 & WISCONSIN & $8,310,732$ & 69 & ALABAMA & $3,396,810$ \\
\hline 13 & CORNELL & $8,036,029$ & 70 & TENNESSEE & $3,322,418$ \\
\hline 14 & WASHINGTON & $7,549,765$ & 71 & MIAMI & $3,300,370$ \\
\hline 15 & PRINCETON & $7,075,441$ & 72 & NEBRASKA & $3,247,311$ \\
\hline 16 & ALBERTA & $7,066,429$ & 73 & CALGARY & $3,244,903$ \\
\hline 17 & MINNESOTA & $6,975,576$ & 74 & SYRACUSE & $3,201,031$ \\
\hline 18 & NORTH CAROLINA & $6,735,325$ & 75 & MONTREAL & $3,180,763$ \\
\hline 19 & BRITISH COLUMBIA & $6,312,477$ & 76 & SOUTHERN ILLINOIS & $3,149,701$ \\
\hline 20 & PENNSYLVANIA & $6,223,214$ & 77 & CALIFORNIA, IRVINE & $3,145,926$ \\
\hline 21 & OHIO STATE & $6,206,443$ & 78 & NEW MEXICO & $3,117,590$ \\
\hline 22 & DUKE & $6,031,761$ & 79 & MIT & $3,057,604$ \\
\hline 23 & PITTSBURGH & $5,897,931$ & 80 & FLORIDA STATE & $3,034,491$ \\
\hline 24 & ARIZONA & $5,794,299$ & 81 & OREGON & $3,006,119$ \\
\hline 25 & VIRGINIA & $5,605,891$ & 82 & CALIFORNIA, SANTA BARBARA & $2,996,397$ \\
\hline 26 & OKLAHOMA & $5,433,036$ & 83 & LAVAL & $2,966,168$ \\
\hline 27 & PENNSYLVANIA STATE & $5,365,489$ & 84 & $\mathrm{OHIO}$ & $2,958,684$ \\
\hline 28 & MICHIGAN STATE & $5,292,806$ & 85 & CALIFORNIA, RIVERSIDE & $2,955,171$ \\
\hline 29 & NEW YORK & $5,191,617$ & 86 & OKLAHOMA STATE & $2,932,910$ \\
\hline 30 & IOWA & $5,155,258$ & 87 & YORK & $2,910,610$ \\
\hline 31 & NORTHWESTERN & $4,930,613$ & 88 & KENT STATE & $2,889,013$ \\
\hline 32 & GEORGIA & $4,716,401$ & 89 & BOSTON & $2,864,562$ \\
\hline 33 & RUTGERS & $4,570,477$ & 90 & DARTMOUTH & $2,848,521$ \\
\hline 34 & ARIZONA STATE & $4,393,156$ & 91 & DELAWARE & $2,807,445$ \\
\hline 35 & COLORADO & $4,348,639$ & 92 & CASE WESTERN RESERVE & $2,777,529$ \\
\hline 36 & FLORIDA & $4,299,252$ & 93 & TEXAS TECH & $2,675,872$ \\
\hline 37 & WASHINGTON U.-ST. LOUIS & $4,281,213$ & 94 & HOUSTON & $2,666,072$ \\
\hline 38 & KANSAS & $4,271,113$ & 95 & RICE & $2,620,342$ \\
\hline 39 & BRIGHAM YOUNG & $4,168,102$ & 96 & HOWARD & $2,589,888$ \\
\hline 40 & NORTH CAROLINA STATE & $4,158,190$ & 97 & IOWA STATE & $2,578,144$ \\
\hline 41 & CALIFORNIA, DAVIS & $4,156,170$ & 98 & QUEEN'S & $2,577,143$ \\
\hline 42 & MCGILL & $4,128,321$ & 99 & BOSTON COLLEGE & $2,555,641$ \\
\hline 43 & SOUTHERN CALIFORNIA & $4,124,253$ & 100 & GEORGIA TECH & $2,541,880$ \\
\hline 44 & LOUISIANA STATE & $4,112,774$ & 101 & ILLINOIS, CHICAGO & $2,515,007$ \\
\hline 45 & TEXAS A\&M & $4,088,969$ & 102 & PURDUE & $2,506,059$ \\
\hline 46 & TULANE & $4,004,458$ & 103 & WASHINGTON STATE & $2,394,849$ \\
\hline 47 & CONNECTICUT & $3,982,991$ & 104 & VIRGINIA TECH & $2,385,815$ \\
\hline 48 & BROWN & $3,936,274$ & 105 & COLORADO STATE & $2,366,608$ \\
\hline 49 & SUNY-BUFFALO & $3,852,074$ & 106 & SASKATCHEWAN & $2,361,589$ \\
\hline 50 & KENTUCKY & $3,784,382$ & 107 & MANITOBA & $2,317,417$ \\
\hline 51 & MARYLAND & $3,767,653$ & 108 & WATERLOO & $2,310,601$ \\
\hline 52 & TEMPLE & $3,761,933$ & 109 & SUNY-STONY BROOK & $2,277,714$ \\
\hline 53 & ROCHESTER & $3,740,714$ & 110 & GEORGE WASHINGTON & $2,268,571$ \\
\hline 54 & JOHNS HOPKINS & $3,737,404$ & 111 & SUNY-ALBANY & $2,263,767$ \\
\hline 55 & CINCINNATI & $3,715,957$ & 112 & LOUISVILLE & $2,234,328$ \\
\hline 56 & SOUTH CAROLINA & $3,675,054$ & 113 & MCMASTER & $2,014,067$ \\
\hline 57 & WAYNE STATE & $3,665,628$ & 114 & GUELPH & $1,898,348$ \\
\hline
\end{tabular}


Rank Order Table 2: Volumes Added (Gross)

\begin{tabular}{|c|c|c|c|c|c|}
\hline RANK & INSTITUTION & Value & RANK & INSTITUTION & VALUE \\
\hline 1 & HARVARD & 349,050 & & RUTGERS & 77,196 \\
\hline 2 & YALE & 292,324 & 59 & GEORGETOWN & 76,926 \\
\hline 3 & ILLINOIS, URBANA & 265,766 & 60 & GEORGE WASHINGTON & 76,171 \\
\hline 4 & CHICAGO & 261,776 & 61 & MIAMI & 72,196 \\
\hline 5 & COLUMBIA & 243,094 & 62 & ILLINOIS, CHICAGO & 69,326 \\
\hline 6 & TORONTO & 239,548 & 63 & HAWAII & 68,517 \\
\hline 7 & PITTSBURGH & 209,556 & 64 & DARTMOUTH & 68,462 \\
\hline 8 & ALBERTA & 191,949 & 65 & LOUISVILLE & 67,550 \\
\hline 9 & MCMASTER & 178,991 & 66 & KENTUCKY & 66,453 \\
\hline 10 & MICHIGAN & 176,363 & 67 & OKLAHOMA STATE & 66,157 \\
\hline 11 & SUNY-BUFFALO & 176,048 & 68 & WASHINGTON U.-ST. LOUIS & 64,505 \\
\hline 12 & IOWA & 174,623 & 69 & TEMPLE & 63,741 \\
\hline 13 & WASHINGTON & 172,772 & 70 & TENNESSEE & 61,859 \\
\hline 14 & TEXAS A\&M & 171,127 & 71 & VIRGINIA TECH & 60,364 \\
\hline 15 & CALIFORNIA, BERKELEY & 162,733 & 72 & SYRACUSE & 60,184 \\
\hline 16 & ARIZONA & 149,544 & 73 & MONTREAL & 58,854 \\
\hline 17 & PRINCETON & 147,989 & 74 & MARYLAND & 57,596 \\
\hline 18 & INDIANA & 146,141 & 75 & SOUTHERN CALIFORNIA & 55,717 \\
\hline 19 & DUKE & 145,399 & 76 & CALIFORNIA, SANTA BARBARA & 55,527 \\
\hline 20 & MINNESOTA & 144,001 & 77 & UTAH & 55,420 \\
\hline 21 & OKLAHOMA & 143,603 & 78 & WASHINGTON STATE & 54,112 \\
\hline 22 & BRITISH COLUMBIA & 142,939 & 79 & MIT & 53,731 \\
\hline 23 & VIRGINIA & 141,305 & 80 & CONNECTICUT & 53,415 \\
\hline 24 & OHIO STATE & 139,870 & 81 & TEXAS TECH & 53,130 \\
\hline 25 & CALIFORNIA, SAN DIEGO & 138,786 & 82 & HOUSTON & 53,041 \\
\hline 26 & NEW YORK & 138,256 & 83 & ARIZONA STATE & 52,991 \\
\hline 27 & NORTH CAROLINA & 137,419 & 84 & SOUTH CAROLINA & 52,810 \\
\hline 28 & PENNSYLVANIA & 135,456 & 85 & VANDERBILT & 52,496 \\
\hline 29 & WISCONSIN & 134,118 & 86 & FLORIDA & 51,159 \\
\hline 30 & TEXAS & 133,562 & 87 & RICE & 50,233 \\
\hline 31 & CALGARY & 130,361 & 88 & NEBRASKA & 50,223 \\
\hline 32 & CORNELL & 130,331 & 89 & OREGON & 49,286 \\
\hline 33 & CALIFORNIA, LOS ANGELES & 127,477 & 90 & SUNY-ALBANY & 48,907 \\
\hline 34 & MCGILL & 121,576 & 91 & IOWA STATE & 48,757 \\
\hline 35 & PENNSYLVANIA STATE & 120,191 & 92 & CALIFORNIA, RIVERSIDE & 47,329 \\
\hline 36 & NEW MEXICO & 119,995 & 93 & GUELPH & 47,313 \\
\hline 37 & TULANE & 114,847 & 94 & BOSTON COLLEGE & 46,551 \\
\hline 38 & BRIGHAM YOUNG & 100,227 & 95 & SASKATCHEWAN & 44,830 \\
\hline 39 & CALIFORNIA, IRVINE & 99,232 & 96 & KANSAS & 44,662 \\
\hline 40 & WESTERN ONTARIO & 99,162 & 97 & MISSOURI & 44,309 \\
\hline 41 & ALABAMA & 92,417 & 98 & FLORIDA STATE & 43,204 \\
\hline 42 & EMORY & 91,504 & 99 & KENT STATE & 42,834 \\
\hline 43 & LAVAL & 89,696 & 100 & QUEEN'S & 42,345 \\
\hline 44 & NORTHWESTERN & 89,452 & 101 & BOSTON & 39,771 \\
\hline 45 & CALIFORNIA, DAVIS & 86,268 & 102 & CASE WESTERN RESERVE & 38,737 \\
\hline 46 & $\mathrm{OHIO}$ & 85,644 & 103 & LOUISIANA STATE & 38,028 \\
\hline 47 & CINCINNATI & 85,635 & 104 & DELAWARE & 37,300 \\
\hline 48 & GEORGIA & 84,117 & 105 & JOHNS HOPKINS & 37,288 \\
\hline 49 & YORK & 84,072 & 106 & WATERLOO & 35,057 \\
\hline 50 & NOTRE DAME & 84,037 & 107 & PURDUE & 33,134 \\
\hline 51 & COLORADO STATE & 83,411 & 108 & ROCHESTER & 31,204 \\
\hline 52 & COLORADO & 82,423 & 109 & SOUTHERN ILLINOIS & 28,048 \\
\hline 53 & MICHIGAN STATE & 81,306 & 110 & AUBURN & 26,083 \\
\hline 54 & MASSACHUSETTS & 80,866 & 111 & WAYNE STATE & 23,678 \\
\hline 55 & MANITOBA & 80,699 & 112 & HOWARD & 22,043 \\
\hline 56 & BROWN & 79,769 & 113 & GEORGIA TECH & 21,489 \\
\hline 57 & NORTH CAROLINA STATE & 78,480 & 114 & SUNY-STONY BROOK & 18,342 \\
\hline
\end{tabular}


Rank Order Table 3: Titles Held

\begin{tabular}{|c|c|c|c|c|c|}
\hline RANK & INSTITUTION & Value & RANK & INSTITUTION & VALUE \\
\hline 1 & MICHIGAN & $9,076,153$ & 58 & CALIFORNIA, DAVIS & $2,136,607$ \\
\hline 2 & YALE & $8,024,854$ & 59 & CASE WESTERN RESERVE & $2,135,777$ \\
\hline 3 & ILLINOIS, URBANA & $7,138,003$ & 60 & LOUISIANA STATE & $2,132,476$ \\
\hline 4 & COLUMBIA & $7,059,240$ & 61 & ILLINOIS, CHICAGO & $2,122,535$ \\
\hline 5 & TORONTO & $6,569,831$ & 62 & NEBRASKA & $2,116,274$ \\
\hline 6 & INDIANA & $5,825,567$ & 63 & DELAWARE & $2,113,639$ \\
\hline 7 & CHICAGO & $5,793,778$ & 64 & EMORY & $2,112,955$ \\
\hline 8 & WISCONSIN & $5,668,964$ & 65 & RICE & $2,109,632$ \\
\hline 9 & ARIZONA & $5,560,453$ & 66 & CALIFORNIA, IRVINE & $2,107,463$ \\
\hline 10 & TEXAS & $5,335,217$ & 67 & CALGARY & $2,088,424$ \\
\hline 11 & PRINCETON & $4,983,404$ & 68 & TEMPLE & $2,065,440$ \\
\hline 12 & CORNELL & $4,963,660$ & 69 & OHIO & $2,052,759$ \\
\hline 13 & MINNESOTA & $4,757,000$ & 70 & BOSTON COLLEGE & $2,045,526$ \\
\hline 14 & IOWA & $4,603,721$ & 71 & NORTH CAROLINA STATE & $2,036,334$ \\
\hline 15 & WASHINGTON & $4,599,418$ & 72 & WASHINGTON STATE & $2,017,135$ \\
\hline 16 & OHIO STATE & $4,540,974$ & 73 & PURDUE & $2,012,865$ \\
\hline 17 & MICHIGAN STATE & $4,396,218$ & 74 & RUTGERS & $2,003,233$ \\
\hline 18 & PENNSYLVANIA STATE & $4,206,341$ & 75 & SOUTHERN ILLINOIS & $1,987,072$ \\
\hline 19 & VIRGINIA & $4,016,275$ & 76 & MONTREAL & $1,956,602$ \\
\hline 20 & KANSAS & $3,815,955$ & 77 & OKLAHOMA STATE & $1,955,273$ \\
\hline 21 & NORTH CAROLINA & $3,757,467$ & 78 & YORK & $1,936,767$ \\
\hline 22 & BRITISH COLUMBIA & $3,726,961$ & 79 & OREGON & $1,885,765$ \\
\hline 23 & GEORGIA & $3,551,110$ & 80 & SYRACUSE & $1,845,727$ \\
\hline 24 & BRIGHAM YOUNG & $3,458,192$ & 81 & MCMASTER & $1,826,224$ \\
\hline 25 & SOUTH CAROLINA & $3,361,206$ & 82 & WATERLOO & $1,807,906$ \\
\hline 26 & ALBERTA & $3,314,868$ & 83 & KENT STATE & $1,735,210$ \\
\hline 27 & OKLAHOMA & $3,271,077$ & 84 & SASKATCHEWAN & $1,714,978$ \\
\hline 28 & AUBURN & $3,136,841$ & 85 & IOWA STATE & $1,609,977$ \\
\hline 29 & TEXAS A\&M & $3,079,479$ & 86 & MANITOBA & $1,578,343$ \\
\hline 30 & NEW YORK & $3,045,051$ & 87 & VIRGINIA TECH & $1,555,134$ \\
\hline 31 & GEORGETOWN & $3,001,874$ & 88 & GUELPH & $1,507,647$ \\
\hline 32 & WASHINGTON U.-ST. LOUIS & $2,984,769$ & 89 & SUNY-ALBANY & $1,463,144$ \\
\hline 33 & CALIFORNIA, SANTA BARBARA & $2,850,203$ & 90 & GEORGE WASHINGTON & $1,460,655$ \\
\hline 34 & ROCHESTER & $2,825,844$ & 91 & LAVAL & $1,366,649$ \\
\hline 35 & MISSOURI & $2,760,594$ & 92 & SUNY-STONY BROOK & $1,284,318$ \\
\hline 36 & MCGILL & $2,703,058$ & 93 & GEORGIA TECH & 798,988 \\
\hline 37 & VANDERBILT & $2,653,228$ & & ARIZONA STATE & $\mathrm{UA} / \mathrm{NA}$ \\
\hline 38 & BROWN & $2,622,422$ & & CALIFORNIA, BERKELEY & $\mathrm{UA} / \mathrm{NA}$ \\
\hline 39 & SOUTHERN CALIFORNIA & $2,579,159$ & & CALIFORNIA, LOS ANGELES & $\mathrm{UA} / \mathrm{NA}$ \\
\hline 40 & ALABAMA & $2,571,076$ & & CALIFORNIA, SAN DIEGO & $\mathrm{UA} / \mathrm{NA}$ \\
\hline 41 & HOUSTON & $2,560,705$ & & COLORADO & $\mathrm{UA} / \mathrm{NA}$ \\
\hline 42 & FLORIDA STATE & $2,505,345$ & & DUKE & $\mathrm{UA} / \mathrm{NA}$ \\
\hline 43 & BOSTON & $2,482,067$ & & FLORIDA & $\mathrm{UA} / \mathrm{NA}$ \\
\hline 44 & SUNY-BUFFALO & $2,421,423$ & & HARVARD & $\mathrm{UA} / \mathrm{NA}$ \\
\hline 45 & NEW MEXICO & $2,414,289$ & & HOWARD & $\mathrm{UA} / \mathrm{NA}$ \\
\hline 46 & WESTERN ONTARIO & $2,410,951$ & & JOHNS HOPKINS & $\mathrm{UA} / \mathrm{NA}$ \\
\hline 47 & NOTRE DAME & $2,396,733$ & & LOUISVILLE & $\mathrm{UA} / \mathrm{NA}$ \\
\hline 48 & CINCINNATI & $2,341,343$ & & MARYLAND & $\mathrm{UA} / \mathrm{NA}$ \\
\hline 49 & MIAMI & $2,331,942$ & & MASSACHUSETTS & $\mathrm{UA} / \mathrm{NA}$ \\
\hline 50 & COLORADO STATE & $2,307,611$ & & MIT & $\mathrm{UA} / \mathrm{NA}$ \\
\hline 51 & CONNECTICUT & $2,304,339$ & & NORTHWESTERN & $\mathrm{UA} / \mathrm{NA}$ \\
\hline 52 & CALIFORNIA, RIVERSIDE & $2,291,248$ & & PENNSYLVANIA & $\mathrm{UA} / \mathrm{NA}$ \\
\hline 53 & KENTUCKY & $2,280,423$ & & PITTSBURGH & $\mathrm{UA} / \mathrm{NA}$ \\
\hline 54 & HAWAII & $2,270,600$ & & QUEEN'S & $\mathrm{UA} / \mathrm{NA}$ \\
\hline 55 & WAYNE STATE & $2,251,350$ & & TEXAS TECH & $\mathrm{UA} / \mathrm{NA}$ \\
\hline 56 & DARTMOUTH & $2,182,194$ & & TULANE & $\mathrm{UA} / \mathrm{NA}$ \\
\hline 57 & TENNESSEE & $2,175,328$ & & UTAH & $\mathrm{UA} / \mathrm{NA}$ \\
\hline
\end{tabular}


Rank Order Table 4: Current Serials (Total)

\begin{tabular}{|c|c|c|c|c|c|}
\hline RANK & INSTITUTION & VAlue & RANK & INSTITUTION & VALUE \\
\hline 1 & COLUMBIA & 144,787 & & KANSAS & 73,613 \\
\hline 2 & HARVARD & 132,107 & 59 & PENNSYLVANIA & 72,688 \\
\hline 3 & VIRGINIA & 117,749 & 60 & IOWA & 72,271 \\
\hline 4 & ILLINOIS, URBANA & 117,517 & 61 & UTAH & 71,926 \\
\hline 5 & ALBERTA & 114,966 & 62 & WASHINGTON U.-ST. LOUIS & 71,905 \\
\hline 6 & NEW YORK & 108,454 & 63 & OKLAHOMA STATE & 70,803 \\
\hline 7 & CALIFORNIA, BERKELEY & 106,108 & 64 & GEORGETOWN & 70,518 \\
\hline 8 & MICHIGAN STATE & 106,026 & 65 & MICHIGAN & 70,047 \\
\hline 9 & LOUISIANA STATE & 104,177 & 66 & WESTERN ONTARIO & 70,023 \\
\hline 10 & CINCINNATI & 103,066 & 67 & QUEEN'S & 69,981 \\
\hline 11 & CHICAGO & 102,767 & 68 & MCMASTER & 69,781 \\
\hline 12 & CORNELL & 102,000 & 69 & OKLAHOMA & 69,621 \\
\hline 13 & WISCONSIN & 101,596 & 70 & CALIFORNIA, SANTA BARBARA & 69,327 \\
\hline 14 & JOHNS HOPKINS & 100,074 & 71 & RUTGERS & 68,560 \\
\hline 15 & PENNSYLVANIA STATE & 99,091 & 72 & HAWAII & 67,863 \\
\hline 16 & NORTHWESTERN & 98,844 & 73 & ILLINOIS, CHICAGO & 67,318 \\
\hline 17 & SOUTHERN CALIFORNIA & 98,728 & 74 & SOUTH CAROLINA & 66,309 \\
\hline 18 & IOWA STATE & 97,352 & 75 & BROWN & 65,908 \\
\hline 19 & TEXAS & 97,206 & 76 & MCGILL & 65,536 \\
\hline 20 & YALE & 96,721 & 77 & DARTMOUTH & 64,745 \\
\hline 21 & GEORGIA & 96,401 & 78 & WASHINGTON & 63,575 \\
\hline 22 & SUNY-STONY BROOK & 95,517 & 79 & NORTH CAROLINA STATE & 63,271 \\
\hline 23 & ARIZONA STATE & 95,515 & 80 & TEMPLE & 62,173 \\
\hline 24 & CONNECTICUT & 92,884 & 81 & COLORADO & 60,805 \\
\hline 25 & DUKE & 91,931 & 82 & PRINCETON & 60,216 \\
\hline 26 & TEXAS A\&M & 91,580 & 83 & MIT & 60,105 \\
\hline 27 & MINNESOTA & 90,860 & 84 & TENNESSEE & 58,377 \\
\hline 28 & MONTREAL & 90,160 & 85 & BOSTON & 57,755 \\
\hline 29 & OHIO STATE & 90,156 & 86 & WAYNE STATE & 56,616 \\
\hline 30 & CALIFORNIA, RIVERSIDE & 90,153 & 87 & LAVAL & 56,527 \\
\hline 31 & FLORIDA & 89,741 & 88 & VANDERBILT & 55,260 \\
\hline 32 & NOTRE DAME & 88,352 & 89 & CALIFORNIA, IRVINE & 53,891 \\
\hline 33 & PITTSBURGH & 87,251 & 90 & MASSACHUSETTS & 53,170 \\
\hline 34 & LOUISVILLE & 86,627 & 91 & COLORADO STATE & 52,433 \\
\hline 35 & INDIANA & 84,933 & 92 & MANITOBA & 52,115 \\
\hline 36 & CALIFORNIA, SAN DIEGO & 84,513 & 93 & MARYLAND & 51,989 \\
\hline 37 & ARIZONA & 84,367 & 94 & GUELPH & 51,566 \\
\hline 38 & TEXAS TECH & 84,317 & 95 & SOUTHERN ILLINOIS & 51,350 \\
\hline 39 & EMORY & 83,514 & 96 & SUNY-ALBANY & 50,693 \\
\hline 40 & TULANE & 83,452 & 97 & AUBURN & 50,011 \\
\hline 41 & TORONTO & 82,413 & 98 & CALGARY & 48,648 \\
\hline 42 & BRITISH COLUMBIA & 82,202 & 99 & PURDUE & 48,283 \\
\hline 43 & ALABAMA & 81,462 & 100 & OREGON & 46,879 \\
\hline 44 & HOUSTON & 81,264 & 101 & $\mathrm{OHIO}$ & 46,823 \\
\hline 45 & RICE & 81,123 & 102 & MISSOURI & 46,543 \\
\hline 46 & NORTH CAROLINA & 80,132 & 103 & GEORGIA TECH & 41,412 \\
\hline 47 & SUNY-BUFFALO & 79,544 & 104 & SASKATCHEWAN & 40,981 \\
\hline 48 & KENT STATE & 79,237 & 105 & NEBRASKA & 40,288 \\
\hline 49 & FLORIDA STATE & 78,295 & 106 & SYRACUSE & 39,703 \\
\hline 50 & KENTUCKY & 78,194 & 107 & CALIFORNIA, LOS ANGELES & 38,975 \\
\hline 51 & BRIGHAM YOUNG & 77,646 & 108 & BOSTON COLLEGE & 37,279 \\
\hline 52 & NEW MEXICO & 77,094 & 109 & ROCHESTER & 36,033 \\
\hline 53 & MIAMI & 76,869 & 110 & WASHINGTON STATE & 35,609 \\
\hline 54 & YORK & 75,381 & 111 & WATERLOO & 35,054 \\
\hline 55 & CASE WESTERN RESERVE & 75,083 & 112 & DELAWARE & 31,706 \\
\hline 56 & GEORGE WASHINGTON & 74,329 & 113 & VIRGINIA TECH & 27,150 \\
\hline 57 & CALIFORNIA, DAVIS & 73,912 & 114 & HOWARD & 24,307 \\
\hline
\end{tabular}


Rank Order Table 5: Microform Units

\begin{tabular}{|c|c|c|c|c|c|}
\hline RANK & INSTITUTION & Value & RANH & InSTITUTION & VALUE \\
\hline 1 & ILLINOIS, URBANA & $10,667,033$ & 58 & BOSTON COLLEGE & $4,226,524$ \\
\hline 2 & YALE & $10,231,747$ & 59 & YORK & $4,225,417$ \\
\hline 3 & HARVARD & $9,997,130$ & 60 & ILLINOIS, CHICAGO & $4,197,939$ \\
\hline 4 & FLORIDA STATE & $9,114,981$ & 61 & PENNSYLVANIA & $4,168,493$ \\
\hline 5 & LOUISIANA STATE & $9,038,682$ & 62 & OREGON & $4,162,266$ \\
\hline 6 & CORNELL & $8,507,500$ & 63 & ALABAMA & $4,102,551$ \\
\hline 7 & MISSOURI & $8,142,874$ & 64 & WESTERN ONTARIO & $4,074,250$ \\
\hline 8 & FLORIDA & $7,919,279$ & 65 & QUEEN'S & $4,059,040$ \\
\hline 9 & WASHINGTON & $7,835,346$ & 66 & MIAMI & $4,030,606$ \\
\hline 10 & ARIZONA STATE & $7,614,192$ & 67 & NOTRE DAME & $4,014,686$ \\
\hline 11 & SYRACUSE & $7,530,901$ & 68 & WASHINGTON STATE & $3,948,577$ \\
\hline 12 & CALIFORNIA, BERKELEY & $7,149,224$ & 69 & WAYNE STATE & $3,898,391$ \\
\hline 13 & COLORADO & $7,031,778$ & 70 & BRIGHAM YOUNG & $3,888,004$ \\
\hline 14 & MINNESOTA & $7,028,582$ & 71 & SUNY-STONY BROOK & $3,848,942$ \\
\hline 15 & MICHIGAN STATE & $6,806,042$ & 72 & CALIFORNIA, SANTA BARBARA & $3,772,031$ \\
\hline 16 & TEXAS & $6,663,065$ & 73 & ALBERTA & $3,744,257$ \\
\hline 17 & GEORGIA & $6,650,817$ & 74 & CALGARY & $3,665,502$ \\
\hline 18 & PRINCETON & $6,612,465$ & 75 & UTAH & $3,641,610$ \\
\hline 19 & KENTUCKY & $6,506,097$ & 76 & IOWA STATE & $3,540,812$ \\
\hline 20 & SOUTHERN CALIFORNIA & $6,415,794$ & 77 & HAWAII & $3,518,804$ \\
\hline 21 & WISCONSIN & $6,412,542$ & 78 & WASHINGTON U.-ST. LOUIS & $3,473,141$ \\
\hline 22 & COLUMBIA & $6,379,196$ & 79 & DELAWARE & $3,447,958$ \\
\hline 23 & VIRGINIA TECH & $6,329,176$ & 80 & CINCINNATI & $3,376,630$ \\
\hline 24 & ARIZONA & $6,308,419$ & 81 & CALIFORNIA, SAN DIEGO & $3,339,220$ \\
\hline 25 & CALIFORNIA, LOS ANGELES & $6,246,884$ & 82 & RICE & $3,328,017$ \\
\hline 26 & SUNY-BUFFALO & $6,150,294$ & 83 & TEMPLE & $3,327,005$ \\
\hline 27 & EMORY & $6,142,110$ & 84 & KANSAS & $3,177,385$ \\
\hline 28 & OHIO STATE & $6,111,550$ & 85 & CHICAGO & $3,147,928$ \\
\hline 29 & RUTGERS & $6,065,247$ & 86 & VANDERBILT & $3,112,303$ \\
\hline 30 & HOUSTON & $5,989,294$ & 87 & PURDUE & $3,102,402$ \\
\hline 31 & MARYLAND & $5,817,532$ & 88 & SASKATCHEWAN & $3,086,526$ \\
\hline 32 & TEXAS A\&M & $5,678,256$ & 89 & SUNY-ALBANY & $2,914,518$ \\
\hline 33 & NEW YORK & $5,592,987$ & 90 & CALIFORNIA, IRVINE & $2,913,560$ \\
\hline 34 & TORONTO & $5,486,050$ & 91 & TEXAS TECH & $2,760,234$ \\
\hline 35 & NORTH CAROLINA STATE & $5,447,358$ & 92 & AUBURN & $2,718,945$ \\
\hline 36 & VIRGINIA & $5,442,787$ & 93 & CASE WESTERN RESERVE & $2,622,179$ \\
\hline 37 & PITTSBURGH & $5,428,310$ & 94 & MASSACHUSETTS & $2,587,145$ \\
\hline 38 & SOUTH CAROLINA & $5,394,547$ & 95 & DARTMOUTH & $2,586,962$ \\
\hline 39 & BRITISH COLUMBIA & $5,324,239$ & 96 & MIT & $2,420,042$ \\
\hline 40 & PENNSYLVANIA STATE & $5,310,696$ & 97 & GUELPH & $2,357,880$ \\
\hline 41 & NORTH CAROLINA & $5,179,705$ & 98 & CALIFORNIA, RIVERSIDE & $2,323,133$ \\
\hline 42 & ROCHESTER & $5,144,222$ & 99 & TULANE & $2,267,026$ \\
\hline 43 & INDIANA & $5,115,583$ & 100 & LOUISVILLE & $2,223,459$ \\
\hline 44 & GEORGETOWN & $5,067,722$ & 101 & OHIO & $1,999,023$ \\
\hline 45 & BOSTON & $4,746,454$ & 102 & BROWN & $1,972,983$ \\
\hline 46 & NEW MEXICO & $4,723,386$ & 103 & MCGILL & $1,796,335$ \\
\hline 47 & NEBRASKA & $4,704,552$ & 104 & WATERLOO & $1,712,333$ \\
\hline 48 & GEORGIA TECH & $4,655,138$ & 105 & MONTREAL & $1,558,155$ \\
\hline 49 & SOUTHERN ILLINOIS & $4,617,239$ & 106 & MCMASTER & $1,517,413$ \\
\hline 50 & NORTHWESTERN & $4,603,214$ & 107 & MANITOBA & $1,474,658$ \\
\hline 51 & OKLAHOMA & $4,476,140$ & 108 & GEORGE WASHINGTON & $1,432,040$ \\
\hline 52 & JOHNS HOPKINS & $4,406,186$ & 109 & LAVAL & $1,309,880$ \\
\hline 53 & TENNESSEE & $4,391,748$ & 110 & COLORADO STATE & $1,158,352$ \\
\hline 54 & DUKE & $4,359,354$ & 111 & OKLAHOMA STATE & 455,806 \\
\hline 55 & CONNECTICUT & $4,342,083$ & & IOWA & $\mathrm{UA} / \mathrm{NA}$ \\
\hline 56 & CALIFORNIA, DAVIS & $4,266,788$ & & KENT STATE & $\mathrm{UA} / \mathrm{NA}$ \\
\hline 57 & HOWARD & $4,262,206$ & & MICHIGAN & $\mathrm{UA} / \mathrm{NA}$ \\
\hline
\end{tabular}


Rank Order Table 6: Government Documents

\begin{tabular}{|c|c|c|c|c|c|}
\hline RANK & INSTITUTION & Value & RANK & INSTITUTION & VALUE \\
\hline 1 & RUTGERS & $2,671,041$ & 58 & CINCINNATI & 28,209 \\
\hline 2 & MINNESOTA & $2,641,702$ & 59 & TEMPLE & 24,883 \\
\hline 3 & MISSOURI & $1,684,682$ & 60 & NORTHWESTERN & 7,808 \\
\hline 4 & NORTH CAROLINA & $1,625,932$ & 61 & TEXAS TECH & 4,222 \\
\hline 5 & DUKE & $1,258,224$ & 62 & TENNESSEE & 4,116 \\
\hline 6 & FLORIDA & $1,246,243$ & 63 & GEORGE WASHINGTON & 3,500 \\
\hline 7 & QUEEN'S & $1,120,094$ & 64 & PRINCETON & 3,071 \\
\hline 8 & GEORGIA & $1,070,308$ & 65 & HOWARD & 2,364 \\
\hline 9 & KENTUCKY & $1,036,135$ & 66 & OHIO STATE & 884 \\
\hline 10 & COLORADO & 955,045 & 67 & ALBERTA & 0 \\
\hline 11 & SOUTH CAROLINA & 951,954 & 67 & ARIZONA & 0 \\
\hline 12 & UTAH & 898,005 & 67 & BRITISH COLUMBIA & 0 \\
\hline 13 & ALABAMA & 884,759 & 67 & BROWN & 0 \\
\hline 14 & FLORIDA STATE & 804,237 & 67 & CALGARY & 0 \\
\hline 15 & ILLINOIS, CHICAGO & 793,177 & 67 & CALIFORNIA, SAN DIEGO & 0 \\
\hline 16 & WISCONSIN & 757,007 & 67 & CONNECTICUT & 0 \\
\hline 17 & KANSAS & 741,660 & 67 & CORNELL & 0 \\
\hline 18 & HOUSTON & 680,971 & 67 & GEORGIA TECH & 0 \\
\hline 19 & MCGILL & 676,933 & 67 & GUELPH & 0 \\
\hline 20 & VIRGINIA & 669,424 & 67 & HAWAII & 0 \\
\hline 21 & ARIZONA STATE & 667,651 & 67 & ILLINOIS, URBANA & 0 \\
\hline 22 & NEW MEXICO & 666,566 & 67 & IOWA STATE & 0 \\
\hline 23 & LOUISIANA STATE & 588,683 & 67 & JOHNS HOPKINS & 0 \\
\hline 24 & NEBRASKA & 552,672 & 67 & KENT STATE & 0 \\
\hline 25 & PURDUE & 484,234 & 67 & MCMASTER & 0 \\
\hline 26 & NEW YORK & 483,140 & 67 & MASSACHUSETTS & 0 \\
\hline 27 & CALIFORNIA, BERKELEY & 465,913 & 67 & MIT & 0 \\
\hline 28 & DELAWARE & 454,050 & 67 & MIAMI & 0 \\
\hline 29 & CALIFORNIA, SANTA BARBARA & 448,265 & 67 & MICHIGAN & 0 \\
\hline 30 & BRIGHAM YOUNG & 420,117 & 67 & MICHIGAN STATE & 0 \\
\hline 31 & ROCHESTER & 419,912 & 67 & MONTREAL & 0 \\
\hline 32 & SASKATCHEWAN & 415,902 & 67 & NORTH CAROLINA STATE & 0 \\
\hline 33 & VIRGINIA TECH & 399,525 & 67 & $\mathrm{OHIO}$ & 0 \\
\hline 34 & WAYNE STATE & 388,182 & 67 & PENNSYLVANIA STATE & 0 \\
\hline 35 & SUNY-STONY BROOK & 348,588 & 67 & PITTSBURGH & 0 \\
\hline 36 & SOUTHERN ILLINOIS & 312,491 & 67 & SUNY-ALBANY & 0 \\
\hline 37 & INDIANA & 311,112 & 67 & SUNY-BUFFALO & 0 \\
\hline 38 & CALIFORNIA, RIVERSIDE & 304,308 & 67 & TEXAS & 0 \\
\hline 39 & SYRACUSE & 293,267 & 67 & WASHINGTON STATE & 0 \\
\hline 40 & SOUTHERN CALIFORNIA & 273,598 & 67 & WASHINGTON U.-ST. LOUIS & 0 \\
\hline 41 & VANDERBILT & 266,061 & 67 & WATERLOO & 0 \\
\hline 42 & OREGON & 248,790 & 67 & WESTERN ONTARIO & 0 \\
\hline 43 & EMORY & 244,737 & 67 & YALE & 0 \\
\hline 44 & AUBURN & 236,917 & & BOSTON & $\mathrm{UA} / \mathrm{NA}$ \\
\hline 45 & MANITOBA & 221,129 & & CHICAGO & $\mathrm{UA} / \mathrm{NA}$ \\
\hline 46 & BOSTON COLLEGE & 219,174 & & COLORADO STATE & $\mathrm{UA} / \mathrm{NA}$ \\
\hline 47 & MARYLAND & 200,367 & & DARTMOUTH & $\mathrm{UA} / \mathrm{NA}$ \\
\hline 48 & OKLAHOMA STATE & 189,681 & & GEORGETOWN & $\mathrm{UA} / \mathrm{NA}$ \\
\hline 49 & COLUMBIA & 174,032 & & HARVARD & $\mathrm{UA} / \mathrm{NA}$ \\
\hline 50 & CALIFORNIA, IRVINE & 149,103 & & IOWA & $\mathrm{UA} / \mathrm{NA}$ \\
\hline 51 & TULANE & 85,069 & & LAVAL & $\mathrm{UA} / \mathrm{NA}$ \\
\hline 52 & CALIFORNIA, LOS ANGELES & 74,779 & & LOUISVILLE & $\mathrm{UA} / \mathrm{NA}$ \\
\hline 53 & CALIFORNIA, DAVIS & 73,387 & & NOTRE DAME & UA/NA \\
\hline 54 & TEXAS A\&M & 65,519 & & PENNSYLVANIA & $\mathrm{UA} / \mathrm{NA}$ \\
\hline 55 & RICE & 55,896 & & TORONTO & $\mathrm{UA} / \mathrm{NA}$ \\
\hline 56 & OKLAHOMA & 41,455 & & WASHINGTON & $\mathrm{UA} / \mathrm{NA}$ \\
\hline 57 & CASE WESTERN RESERVE & 30,653 & & YORK & $\mathrm{UA} / \mathrm{NA}$ \\
\hline
\end{tabular}

Rank Order Tables of University Libraries · 79 
Rank Order Table 7: Total Library Materials Expenditures

\begin{tabular}{|c|c|c|c|c|c|}
\hline RANK & INSTITUTION & VALUE & RANK & INSTITUTION & VALUE \\
\hline 1 & YALE & $39,105,895$ & 58 & WESTERN ONTARIO & $10,185,101$ \\
\hline 2 & HARVARD & $35,296,483$ & 59 & CINCINNATI & $10,179,240$ \\
\hline 3 & COLUMBIA & $24,555,907$ & 60 & CONNECTICUT & $10,158,424$ \\
\hline 4 & TORONTO & $23,309,826$ & 61 & YORK & $9,911,392$ \\
\hline 5 & MICHIGAN & $22,565,018$ & 62 & HOUSTON & $9,903,271$ \\
\hline 6 & PRINCETON & $21,934,491$ & 63 & CALIFORNIA, SAN DIEGO & $9,876,233$ \\
\hline 7 & PENNSYLVANIA STATE & $18,494,217$ & 64 & NORTH CAROLINA STATE & $9,676,210$ \\
\hline 8 & CHICAGO & $18,073,119$ & 65 & LAVAL & $9,632,798$ \\
\hline 9 & NEW YORK & $17,918,458$ & 66 & KANSAS & $9,621,889$ \\
\hline 10 & CALIFORNIA, BERKELEY & $17,849,390$ & 67 & SASKATCHEWAN & $9,602,049$ \\
\hline 11 & TEXAS A\&M & $17,768,690$ & 68 & BRIGHAM YOUNG & $9,542,790$ \\
\hline 12 & TEXAS & $17,427,074$ & 69 & MARYLAND & $9,499,472$ \\
\hline 13 & SOUTHERN CALIFORNIA & $17,354,171$ & 70 & CALIFORNIA, IRVINE & $9,489,506$ \\
\hline 14 & MINNESOTA & $16,999,808$ & 71 & LOUISVILLE & $9,487,675$ \\
\hline 15 & EMORY & $16,977,757$ & 72 & DARTMOUTH & $9,399,296$ \\
\hline 16 & ALBERTA & $16,761,357$ & 73 & BOSTON & $9,375,336$ \\
\hline 17 & WASHINGTON & $16,657,209$ & 74 & BOSTON COLLEGE & $9,161,518$ \\
\hline 18 & DUKE & $16,451,465$ & 75 & MIT & $9,051,913$ \\
\hline 19 & NORTH CAROLINA & $16,167,925$ & 76 & DELAWARE & $8,969,172$ \\
\hline 20 & CORNELL & $16,161,049$ & 77 & SUNY-BUFFALO & $8,951,888$ \\
\hline 21 & BRITISH COLUMBIA & $15,366,604$ & 78 & BROWN & $8,817,845$ \\
\hline 22 & PENNSYLVANIA & $15,224,637$ & 79 & TULANE & $8,749,315$ \\
\hline 23 & INDIANA & $14,794,891$ & 80 & CALIFORNIA, DAVIS & $8,717,848$ \\
\hline 24 & JOHNS HOPKINS & $14,572,377$ & 81 & WAYNE STATE & $8,701,072$ \\
\hline 25 & ILLINOIS, URBANA & $14,529,554$ & 82 & MISSOURI & $8,459,261$ \\
\hline 26 & CALIFORNIA, LOS ANGELES & $14,459,035$ & 83 & QUEEN'S & $8,328,121$ \\
\hline 27 & OKLAHOMA & $14,406,308$ & 84 & ROCHESTER & $8,266,771$ \\
\hline 28 & IOWA & $14,278,353$ & 85 & ALABAMA & $8,207,066$ \\
\hline 29 & PITTSBURGH & $14,206,270$ & 86 & SOUTH CAROLINA & $8,144,704$ \\
\hline 30 & NORTHWESTERN & $13,900,682$ & 87 & HAWAII & $8,105,607$ \\
\hline 31 & MIAMI & $13,694,535$ & 88 & FLORIDA STATE & $8,062,835$ \\
\hline 32 & VIRGINIA & $13,107,062$ & 89 & NEW MEXICO & $7,989,009$ \\
\hline 33 & MCGILL & $12,989,455$ & 90 & MCMASTER & $7,981,505$ \\
\hline 34 & ARIZONA & $12,890,572$ & 91 & MANITOBA & $7,756,008$ \\
\hline 35 & WASHINGTON U.-ST. LOUIS & $12,772,285$ & 92 & SYRACUSE & $7,571,297$ \\
\hline 36 & OHIO STATE & $12,667,516$ & 93 & UTAH & $7,314,858$ \\
\hline 37 & IOWA STATE & $12,560,760$ & 94 & SOUTHERN ILLINOIS & $7,271,572$ \\
\hline 38 & FLORIDA & $12,261,949$ & 95 & VIRGINIA TECH & $7,155,113$ \\
\hline 39 & MONTREAL & $12,247,451$ & 96 & SUNY-STONY BROOK & $6,950,740$ \\
\hline 40 & TENNESSEE & $12,146,743$ & 97 & OKLAHOMA STATE & $6,912,637$ \\
\hline 41 & GEORGETOWN & $12,010,333$ & 98 & CASE WESTERN RESERVE & $6,707,114$ \\
\hline 42 & TEMPLE & $11,942,661$ & 99 & COLORADO STATE & $6,509,389$ \\
\hline 43 & CALGARY & $11,788,750$ & 100 & NEBRASKA & $6,458,844$ \\
\hline 44 & MICHIGAN STATE & $11,772,095$ & 101 & MASSACHUSETTS & $6,417,445$ \\
\hline 45 & NOTRE DAME & $11,736,827$ & 102 & OREGON & $6,387,091$ \\
\hline 46 & WISCONSIN & $11,710,988$ & 103 & LOUISIANA STATE & $6,323,242$ \\
\hline 47 & TEXAS TECH & $11,514,413$ & 104 & WASHINGTON STATE & $6,152,879$ \\
\hline 48 & RUTGERS & $11,479,410$ & 105 & GEORGIA TECH & $6,037,151$ \\
\hline 49 & COLORADO & $11,394,102$ & 106 & WATERLOO & $5,925,083$ \\
\hline 50 & PURDUE & $11,225,286$ & 107 & GUELPH & $5,818,580$ \\
\hline 51 & ARIZONA STATE & $11,204,849$ & 108 & AUBURN & $5,760,251$ \\
\hline 52 & GEORGE WASHINGTON & $11,175,276$ & 109 & OHIO & $5,464,827$ \\
\hline 53 & VANDERBILT & $11,063,708$ & 110 & CALIFORNIA, SANTA BARBARA & $5,449,640$ \\
\hline 54 & KENTUCKY & $10,769,278$ & 111 & SUNY-ALBANY & $5,198,901$ \\
\hline 55 & ILLINOIS, CHICAGO & $10,686,348$ & 112 & CALIFORNIA, RIVERSIDE & $4,802,770$ \\
\hline 56 & RICE & $10,460,929$ & 113 & KENT STATE & $4,203,944$ \\
\hline 57 & GEORGIA & $10,364,778$ & 114 & HOWARD & $4,073,010$ \\
\hline
\end{tabular}


Rank Order Table 8: Total Salaries \& Wages Expenditures

\begin{tabular}{|c|c|c|c|c|c|}
\hline RANK & INSTITUTION & Value & RANK & INSTITUTION & VAlue \\
\hline 1 & HARVARD & $65,459,316$ & 58 & WASHINGTON U.-ST. LOUIS & $10,453,326$ \\
\hline 2 & YALE & $37,963,400$ & 59 & GEORGIA & $10,399,193$ \\
\hline 3 & TORONTO & $32,014,667$ & 60 & TEXAS TECH & $10,383,218$ \\
\hline 4 & COLUMBIA & $31,150,897$ & 61 & VANDERBILT & $10,239,563$ \\
\hline 5 & CALIFORNIA, BERKELEY & $29,613,101$ & 62 & CALIFORNIA, IRVINE & $10,183,657$ \\
\hline 6 & CALIFORNIA, LOS ANGELES & $27,895,397$ & 63 & CALIFORNIA, DAVIS & $10,072,737$ \\
\hline 7 & MICHIGAN & $25,797,543$ & 64 & HAWAII & $9,995,960$ \\
\hline 8 & PENNSYLVANIA STATE & $25,412,735$ & 65 & BROWN & $9,843,036$ \\
\hline 9 & CORNELL & $23,594,158$ & 66 & LAVAL & $9,840,839$ \\
\hline 10 & WISCONSIN & $23,504,056$ & 67 & MIAMI & $9,671,749$ \\
\hline 11 & WASHINGTON & $22,724,950$ & 68 & BOSTON & $9,652,315$ \\
\hline 12 & TEXAS & $22,419,353$ & 69 & TENNESSEE & $9,623,443$ \\
\hline 13 & ILLINOIS, URBANA & $21,860,269$ & 70 & WAYNE STATE & $9,398,088$ \\
\hline 14 & NEW YORK & $20,886,219$ & 71 & BOSTON COLLEGE & $8,989,212$ \\
\hline 15 & NORTH CAROLINA & $20,246,162$ & 72 & COLORADO & $8,961,047$ \\
\hline 16 & PRINCETON & $19,654,018$ & 73 & SYRACUSE & $8,820,520$ \\
\hline 17 & RUTGERS & $19,603,434$ & 74 & DARTMOUTH & $8,691,301$ \\
\hline 18 & MINNESOTA & $18,578,936$ & 75 & KENTUCKY & $8,527,580$ \\
\hline 19 & PENNSYLVANIA & $17,737,813$ & 76 & OKLAHOMA STATE & $8,431,689$ \\
\hline 20 & OHIO STATE & $17,605,603$ & 77 & TEMPLE & $8,341,799$ \\
\hline 21 & DUKE & $17,278,906$ & 78 & OREGON & $8,294,190$ \\
\hline 22 & SOUTHERN CALIFORNIA & $17,135,114$ & 79 & DELAWARE & $8,012,462$ \\
\hline 23 & VIRGINIA & $16,911,793$ & 80 & ROCHESTER & $8,010,346$ \\
\hline 24 & ALBERTA & $16,809,481$ & 81 & ILLINOIS, CHICAGO & $7,984,514$ \\
\hline 25 & CALIFORNIA, SAN DIEGO & $16,596,674$ & 82 & CINCINNATI & $7,891,184$ \\
\hline 26 & MONTREAL & $16,514,087$ & 83 & WESTERN ONTARIO & $7,694,513$ \\
\hline 27 & INDIANA & $15,505,892$ & 84 & SOUTH CAROLINA & $7,631,053$ \\
\hline 28 & BRITISH COLUMBIA & $15,471,461$ & 85 & MASSACHUSETTS & $7,565,947$ \\
\hline 29 & CONNECTICUT & $14,788,200$ & 86 & IOWA STATE & $7,460,153$ \\
\hline 30 & FLORIDA & $13,765,685$ & 87 & QUEEN'S & $7,330,873$ \\
\hline 31 & TEXAS A\&M & $13,523,064$ & 88 & CALIFORNIA, RIVERSIDE & $7,294,718$ \\
\hline 32 & EMORY & $13,501,946$ & 89 & NEBRASKA & $7,287,801$ \\
\hline 33 & IOWA & $13,455,044$ & 90 & FLORIDA STATE & $7,142,885$ \\
\hline 34 & CHICAGO & $13,316,454$ & 91 & MCMASTER & $7,106,305$ \\
\hline 35 & NORTHWESTERN & $13,291,080$ & 92 & SASKATCHEWAN & $7,038,186$ \\
\hline 36 & MARYLAND & $12,938,368$ & 93 & OKLAHOMA & $7,002,375$ \\
\hline 37 & PITTSBURGH & $12,882,043$ & 94 & MISSOURI & $6,963,818$ \\
\hline 38 & MIT & $12,788,537$ & 95 & ALABAMA & $6,862,189$ \\
\hline 39 & BRIGHAM YOUNG & $12,757,542$ & 96 & WASHINGTON STATE & $6,857,653$ \\
\hline 40 & GEORGETOWN & $12,700,584$ & 97 & SUNY-ALBANY & $6,725,104$ \\
\hline 41 & CALGARY & $12,552,456$ & 98 & SOUTHERN ILLINOIS & $6,582,192$ \\
\hline 42 & NORTH CAROLINA STATE & $12,177,807$ & 99 & LOUISVILLE & $6,364,637$ \\
\hline 43 & ARIZONA & $11,902,505$ & 100 & HOUSTON & $6,297,022$ \\
\hline 44 & MICHIGAN STATE & $11,899,012$ & 101 & LOUISIANA STATE & $6,220,993$ \\
\hline 45 & UTAH & $11,778,069$ & 102 & COLORADO STATE & $6,190,042$ \\
\hline 46 & ARIZONA STATE & $11,261,571$ & 103 & WATERLOO & $6,170,048$ \\
\hline 47 & GEORGE WASHINGTON & $11,083,140$ & 104 & SUNY-STONY BROOK & $6,121,945$ \\
\hline 48 & CALIFORNIA, SANTA BARBARA & $11,074,343$ & 105 & OHIO & $6,043,461$ \\
\hline 49 & MCGILL & $11,016,091$ & 106 & VIRGINIA TECH & $5,824,624$ \\
\hline 50 & JOHNS HOPKINS & $10,923,211$ & 107 & CASE WESTERN RESERVE & $5,800,376$ \\
\hline 51 & PURDUE & $10,909,641$ & 108 & GUELPH & $5,707,282$ \\
\hline 52 & KANSAS & $10,844,666$ & 109 & GEORGIA TECH & $5,403,557$ \\
\hline 53 & NOTRE DAME & $10,818,372$ & 110 & TULANE & $5,368,877$ \\
\hline 54 & YORK & $10,675,456$ & 111 & KENT STATE & $5,329,723$ \\
\hline 55 & MANITOBA & $10,513,384$ & 112 & AUBURN & $5,088,887$ \\
\hline 56 & NEW MEXICO & $10,463,011$ & 113 & RICE & $4,957,985$ \\
\hline 57 & SUNY-BUFFALO & $10,462,236$ & 114 & HOWARD & $4,703,429$ \\
\hline
\end{tabular}

Rank Order Tables of University Libraries $\cdot 81$ 
Rank Order Table 9: Other Operating Expenditures

\begin{tabular}{|c|c|c|c|c|c|}
\hline RANK & INSTITUTION & Value & RANK & INSTITUTION & VAlue \\
\hline 1 & HARVARD & $21,200,914$ & 58 & OKLAHOMASTATE & $2,509,742$ \\
\hline 2 & YALE & $11,352,128$ & 59 & TEMPLE & $2,459,950$ \\
\hline 3 & ALBERTA & $8,697,279$ & 60 & KANSAS & $2,458,941$ \\
\hline 4 & WISCONSIN & $8,309,231$ & 61 & NOTRE DAME & $2,360,654$ \\
\hline 5 & OHIO STATE & $8,201,238$ & 62 & MCMASTER & $2,355,226$ \\
\hline 6 & CALIFORNIA, LOS ANGELES & $7,477,952$ & 63 & KENTUCKY & $2,327,502$ \\
\hline 7 & COLUMBIA & $6,900,223$ & 64 & BOSTON & $2,315,914$ \\
\hline 8 & CORNELL & $6,638,001$ & 65 & ALABAMA & $2,313,804$ \\
\hline 9 & NEW YORK & $6,579,356$ & 66 & BROWN & $2,279,701$ \\
\hline 10 & NORTH CAROLINA STATE & $6,546,495$ & 67 & OKLAHOMA & $2,237,943$ \\
\hline 11 & PENNSYLVANIA & $6,344,431$ & 68 & CALIFORNIA, IRVINE & $2,224,888$ \\
\hline 12 & TEXAS & $6,175,281$ & 69 & NEW MEXICO & $2,189,493$ \\
\hline 13 & ARIZONA & $5,819,286$ & 70 & ARIZONA STATE & $2,125,271$ \\
\hline 14 & PRINCETON & $5,500,247$ & 71 & CINCINNATI & $2,124,416$ \\
\hline 15 & MINNESOTA & $5,395,625$ & 72 & RUTGERS & $2,050,656$ \\
\hline 16 & PENNSYLVANIA STATE & $5,196,367$ & 73 & FLORIDA & $2,017,776$ \\
\hline 17 & TEXAS A\&M & $4,983,210$ & 74 & ILLINOIS, CHICAGO & $1,913,357$ \\
\hline 18 & TORONTO & $4,972,166$ & 75 & SYRACUSE & $1,896,018$ \\
\hline 19 & ILLINOIS, URBANA & $4,943,339$ & 76 & MANITOBA & $1,832,792$ \\
\hline 20 & WASHINGTON U.-ST. LOUIS & $4,908,148$ & 77 & GEORGIA & $1,771,984$ \\
\hline 21 & UTAH & $4,782,547$ & 78 & CONNECTICUT & $1,728,705$ \\
\hline 22 & MICHIGAN & $4,392,321$ & 79 & OHIO & $1,724,039$ \\
\hline 23 & WASHINGTON & $4,366,816$ & 80 & MISSOURI & $1,721,278$ \\
\hline 24 & SOUTHERN CALIFORNIA & $4,364,993$ & 81 & COLORADO & $1,706,513$ \\
\hline 25 & MCGILL & $4,170,119$ & 82 & DELAWARE & $1,700,375$ \\
\hline 26 & VIRGINIA & $4,139,609$ & 83 & TENNESSEE & $1,683,631$ \\
\hline 27 & EMORY & $4,133,319$ & 84 & IOWA & $1,623,439$ \\
\hline 28 & NORTH CAROLINA & $4,127,565$ & 85 & SUNY-BUFFALO & $1,570,492$ \\
\hline 29 & GEORGE WASHINGTON & $4,112,144$ & 86 & DARTMOUTH & $1,548,655$ \\
\hline 30 & DUKE & $4,099,020$ & 87 & SASKATCHEWAN & $1,540,740$ \\
\hline 31 & TEXAS TECH & $4,078,135$ & 88 & CASE WESTERN RESERVE & $1,464,064$ \\
\hline 32 & BRITISH COLUMBIA & $3,925,473$ & 89 & BOSTON COLLEGE & $1,409,680$ \\
\hline 33 & CHICAGO & $3,920,017$ & 90 & NEBRASKA & $1,381,865$ \\
\hline 34 & HOUSTON & $3,843,663$ & 91 & AUBURN & $1,373,732$ \\
\hline 35 & LOUISVILLE & $3,560,918$ & 92 & MASSACHUSETTS & $1,350,309$ \\
\hline 36 & JOHNS HOPKINS & $3,560,865$ & 93 & OREGON & $1,326,614$ \\
\hline 37 & BRIGHAM YOUNG & $3,469,781$ & 94 & WASHINGTON STATE & $1,299,060$ \\
\hline 38 & CALIFORNIA, BERKELEY & $3,449,351$ & 95 & WATERLOO & $1,275,001$ \\
\hline 39 & SOUTH CAROLINA & $3,420,807$ & 96 & SOUTHERN ILLINOIS & $1,264,100$ \\
\hline 40 & CALIFORNIA, SAN DIEGO & $3,386,044$ & 97 & CALIFORNIA, RIVERSIDE & $1,258,350$ \\
\hline 41 & MARYLAND & $3,249,588$ & 98 & QUEEN'S & $1,217,313$ \\
\hline 42 & MIT & $3,218,187$ & 99 & VIRGINIA TECH & $1,144,668$ \\
\hline 43 & GEORGETOWN & $3,217,227$ & 100 & CALGARY & $1,099,731$ \\
\hline 44 & MIAMI & $3,144,000$ & 101 & GEORGIA TECH & $1,063,027$ \\
\hline 45 & IOWA STATE & $3,123,705$ & 102 & CALIFORNIA, DAVIS & $1,010,819$ \\
\hline 46 & VANDERBILT & $2,991,816$ & 103 & GUELPH & 946,003 \\
\hline 47 & KENT STATE & $2,944,949$ & 104 & MONTREAL & 910,783 \\
\hline 48 & WAYNE STATE & $2,924,935$ & 105 & LOUISIANA STATE & 841,535 \\
\hline 49 & PITTSBURGH & $2,911,601$ & 106 & RICE & 778,187 \\
\hline 50 & YORK & $2,910,774$ & 107 & HAWAII & 766,420 \\
\hline 51 & NORTHWESTERN & $2,857,200$ & 108 & SUNY-STONY BROOK & 673,702 \\
\hline 52 & INDIANA & $2,693,223$ & 109 & WESTERN ONTARIO & 641,522 \\
\hline 53 & PURDUE & $2,648,370$ & 110 & TULANE & 637,428 \\
\hline 54 & COLORADO STATE & $2,597,665$ & 111 & LAVAL & 574,607 \\
\hline 55 & CALIFORNIA, SANTA BARBARA & $2,547,301$ & 112 & HOWARD & 553,566 \\
\hline 56 & MICHIGAN STATE & $2,540,397$ & 113 & SUNY-ALBANY & 541,643 \\
\hline 57 & ROCHESTER & $2,520,104$ & 114 & FLORIDA STATE & 495,777 \\
\hline
\end{tabular}


Rank Order Table 10: Total Library Expenditures

\begin{tabular}{|c|c|c|c|c|c|}
\hline RANK & INSTITUTION & VAlue & RANK & INSTITUTION & Value \\
\hline 1 & HARVARD & $122,980,135$ & 58 & TENNESSEE & $23,515,580$ \\
\hline 2 & YALE & $88,942,761$ & 59 & IOWA STATE & $23,250,210$ \\
\hline 3 & COLUMBIA & $63,320,237$ & 60 & KANSAS & $23,042,922$ \\
\hline 4 & TORONTO & $60,605,439$ & 61 & TEMPLE & $22,861,433$ \\
\hline 5 & MICHIGAN & $53,134,323$ & 62 & GEORGIA & $22,802,390$ \\
\hline 6 & CALIFORNIA, BERKELEY & $51,973,099$ & 63 & COLORADO & $22,131,757$ \\
\hline 7 & CALIFORNIA, LOS ANGELES & $50,205,645$ & 64 & CALIFORNIA, IRVINE & $21,947,912$ \\
\hline 8 & PENNSYLVANIA STATE & $49,451,130$ & 65 & KENTUCKY & $21,734,374$ \\
\hline 9 & PRINCETON & $47,636,724$ & 66 & BOSTON & $21,486,285$ \\
\hline 10 & CORNELL & $46,546,720$ & 67 & WAYNE STATE & $21,090,767$ \\
\hline 11 & TEXAS & $46,205,263$ & 68 & SUNY-BUFFALO & $21,026,650$ \\
\hline 12 & NEW YORK & $45,893,186$ & 69 & BROWN & $21,001,345$ \\
\hline 13 & WASHINGTON & $43,956,131$ & 70 & NEW MEXICO & $20,809,647$ \\
\hline 14 & WISCONSIN & $43,732,756$ & 71 & ILLINOIS, CHICAGO & $20,616,384$ \\
\hline 15 & ALBERTA & $42,330,195$ & 72 & CINCINNATI & $20,330,143$ \\
\hline 16 & ILLINOIS, URBANA & $41,600,882$ & 73 & MANITOBA & $20,232,942$ \\
\hline 17 & MINNESOTA & $41,225,580$ & 74 & HOUSTON & $20,149,427$ \\
\hline 18 & NORTH CAROLINA & $40,769,990$ & 75 & LAVAL & $20,099,031$ \\
\hline 19 & PENNSYLVANIA & $39,587,076$ & 76 & CALIFORNIA, DAVIS & $19,932,904$ \\
\hline 20 & SOUTHERN CALIFORNIA & $38,993,308$ & 77 & DARTMOUTH & $19,702,562$ \\
\hline 21 & OHIO STATE & $38,818,365$ & 78 & BOSTON COLLEGE & $19,637,700$ \\
\hline 22 & DUKE & $38,013,641$ & 79 & LOUISVILLE & $19,533,056$ \\
\hline 23 & TEXAS A\&M & $36,374,551$ & 80 & SOUTH CAROLINA & $19,284,992$ \\
\hline 24 & CHICAGO & $35,646,147$ & 81 & CALIFORNIA, SANTA BARBARA & $19,197,432$ \\
\hline 25 & BRITISH COLUMBIA & $34,925,597$ & 82 & HAWAII & $19,076,939$ \\
\hline 26 & EMORY & $34,720,327$ & 83 & ROCHESTER & $18,887,593$ \\
\hline 27 & VIRGINIA & $34,248,073$ & 84 & DELAWARE & $18,734,280$ \\
\hline 28 & RUTGERS & $33,219,035$ & 85 & WESTERN ONTARIO & $18,632,779$ \\
\hline 29 & INDIANA & $33,147,603$ & 86 & SYRACUSE & $18,340,174$ \\
\hline 30 & ARIZONA & $30,645,753$ & 87 & SASKATCHEWAN & $18,235,214$ \\
\hline 31 & NORTHWESTERN & $30,270,763$ & 88 & OKLAHOMA STATE & $17,886,909$ \\
\hline 32 & PITTSBURGH & $30,145,301$ & 89 & MCMASTER & $17,449,235$ \\
\hline 33 & CALIFORNIA, SAN DIEGO & $30,076,158$ & 90 & ALABAMA & $17,430,914$ \\
\hline 34 & MONTREAL & $29,812,219$ & 91 & MISSOURI & $17,235,752$ \\
\hline 35 & IOWA & $29,517,181$ & 92 & QUEEN'S & $16,939,189$ \\
\hline 36 & JOHNS HOPKINS & $29,124,364$ & 93 & RICE & $16,246,764$ \\
\hline 37 & NORTH CAROLINA STATE & $28,483,805$ & 94 & OREGON & $16,074,716$ \\
\hline 38 & MCGILL & $28,349,450$ & 95 & FLORIDA STATE & $15,750,486$ \\
\hline 39 & WASHINGTON U.-ST. LOUIS & $28,230,503$ & 96 & MASSACHUSETTS & $15,403,001$ \\
\hline 40 & FLORIDA & $28,147,202$ & 97 & COLORADO STATE & $15,334,082$ \\
\hline 41 & GEORGETOWN & $28,040,057$ & 98 & NEBRASKA & $15,230,621$ \\
\hline 42 & CONNECTICUT & $26,831,009$ & 99 & SOUTHERN ILLINOIS & $15,228,311$ \\
\hline 43 & MIAMI & $26,584,581$ & 100 & TULANE & $14,875,907$ \\
\hline 44 & GEORGE WASHINGTON & $26,491,902$ & 101 & WASHINGTON STATE & $14,356,836$ \\
\hline 45 & MICHIGAN STATE & $26,411,564$ & 102 & VIRGINIA TECH & $14,177,077$ \\
\hline 46 & TEXAS TECH & $26,049,443$ & 103 & CASE WESTERN RESERVE & $14,019,260$ \\
\hline 47 & BRIGHAM YOUNG & $25,894,552$ & 104 & SUNY-STONY BROOK & $13,772,703$ \\
\hline 48 & MARYLAND & $25,783,433$ & 105 & LOUISIANA STATE & $13,428,011$ \\
\hline 49 & CALGARY & $25,515,738$ & 106 & WATERLOO & $13,420,234$ \\
\hline 50 & MIT & $25,260,636$ & 107 & CALIFORNIA, RIVERSIDE & $13,395,277$ \\
\hline 51 & NOTRE DAME & $24,999,298$ & 108 & OHIO & $13,268,842$ \\
\hline 52 & PURDUE & $24,830,291$ & 109 & KENT STATE & $12,551,309$ \\
\hline 53 & ARIZONA STATE & $24,640,095$ & 110 & GEORGIA TECH & $12,511,623$ \\
\hline 54 & VANDERBILT & $24,395,492$ & 111 & SUNY-ALBANY & $12,506,329$ \\
\hline 55 & UTAH & $23,948,377$ & 112 & GUELPH & $12,495,731$ \\
\hline 56 & OKLAHOMA & $23,865,613$ & 113 & AUBURN & $12,276,211$ \\
\hline 57 & YORK & $23,622,720$ & 114 & HOWARD & $9,347,005$ \\
\hline
\end{tabular}


Rank Order Table 11: Monographs Purchased (Volumes)

\begin{tabular}{|c|c|c|c|c|c|}
\hline RANK & INSTITUTION & VALUE & RANK & InSTITUTION & VALUE \\
\hline 1 & TULANE & 638,592 & & CINCINNATI & 34,035 \\
\hline 2 & YALE & 206,555 & 59 & KENTUCKY & 33,763 \\
\hline 3 & MCGILL & 201,983 & 60 & MISSOURI & 33,643 \\
\hline 4 & COLUMBIA & 167,270 & 61 & ARIZONA STATE & 32,851 \\
\hline 5 & TORONTO & 158,826 & 62 & WATERLOO & 32,253 \\
\hline 6 & TEXAS A\&M & 144,623 & 63 & LOUISIANA STATE & 30,989 \\
\hline 7 & MICHIGAN & 144,173 & 64 & WASHINGTON U.-ST. LOUIS & 30,637 \\
\hline 8 & ILLINOIS, URBANA & 132,645 & 65 & FLORIDA & 29,994 \\
\hline 9 & CHICAGO & 129,636 & 66 & GEORGE WASHINGTON & 29,593 \\
\hline 10 & PRINCETON & 123,349 & 67 & $\mathrm{OHIO}$ & 28,737 \\
\hline 11 & MANITOBA & 123,082 & 68 & IOWA STATE & 28,369 \\
\hline 12 & MARYLAND & 117,610 & 69 & JOHNS HOPKINS & 28,280 \\
\hline 13 & ALABAMA & 98,779 & 70 & BOSTON COLLEGE & 28,152 \\
\hline 14 & INDIANA & 84,088 & 71 & HAWAII & 25,801 \\
\hline 15 & DUKE & 78,817 & 72 & YORK & 25,659 \\
\hline 16 & WISCONSIN & 77,018 & 73 & NEW MEXICO & 25,253 \\
\hline 17 & OHIO STATE & 73,102 & 74 & QUEEN'S & 23,587 \\
\hline 18 & BRIGHAM YOUNG & 72,501 & 75 & MICHIGAN STATE & 23,397 \\
\hline 19 & COLORADO STATE & 70,125 & 76 & SUNY-BUFFALO & 23,160 \\
\hline 20 & NORTH CAROLINA & 67,691 & 77 & VANDERBILT & 22,963 \\
\hline 21 & VIRGINIA & 65,765 & 78 & DELAWARE & 22,798 \\
\hline 22 & CALIFORNIA, DAVIS & 65,603 & 79 & MIT & 22,669 \\
\hline 23 & COLORADO & 64,165 & 80 & SYRACUSE & 21,678 \\
\hline 24 & WASHINGTON & 62,391 & 81 & NORTH CAROLINA STATE & 21,544 \\
\hline 25 & TEXAS & 61,470 & 82 & RUTGERS & 21,378 \\
\hline 26 & MONTREAL & 61,439 & 83 & ROCHESTER & 21,022 \\
\hline 27 & PENNSYLVANIA STATE & 60,919 & 84 & HOUSTON & 20,009 \\
\hline 28 & OKLAHOMA STATE & 57,964 & 85 & CALIFORNIA, SANTA BARBARA & 19,509 \\
\hline 29 & DARTMOUTH & 54,650 & 86 & BOSTON & 19,425 \\
\hline 30 & MCMASTER & 53,891 & 87 & VIRGINIA TECH & 18,119 \\
\hline 31 & SOUTHERN CALIFORNIA & 53,645 & 88 & FLORIDA STATE & 17,143 \\
\hline 32 & IOWA & 52,154 & 89 & SASKATCHEWAN & 17,050 \\
\hline 33 & NEW YORK & 51,828 & 90 & WAYNE STATE & 16,889 \\
\hline 34 & WESTERN ONTARIO & 50,990 & 91 & OREGON & 16,761 \\
\hline 35 & CALIFORNIA, LOS ANGELES & 50,170 & 92 & SUNY-ALBANY & 16,065 \\
\hline 36 & EMORY & 49,794 & 93 & CONNECTICUT & 15,673 \\
\hline 37 & NORTHWESTERN & 47,683 & 94 & KENT STATE & 14,948 \\
\hline 38 & TENNESSEE & 45,399 & 95 & SOUTH CAROLINA & 14,256 \\
\hline 39 & NOTRE DAME & 45,172 & 96 & PURDUE & 13,774 \\
\hline 40 & BROWN & 44,711 & 97 & WASHINGTON STATE & 13,767 \\
\hline 41 & CALGARY & 44,137 & 98 & SOUTHERN ILLINOIS & 13,338 \\
\hline 42 & OKLAHOMA & 43,015 & 99 & GUELPH & 12,992 \\
\hline 43 & GEORGIA & 41,517 & 100 & CALIFORNIA, RIVERSIDE & 11,849 \\
\hline 44 & MIAMI & 40,438 & 101 & MASSACHUSETTS & 11,286 \\
\hline 45 & RICE & 40,286 & 102 & AUBURN & 11,277 \\
\hline 46 & ILLINOIS, CHICAGO & 40,237 & 103 & NEBRASKA & 9,923 \\
\hline 47 & LAVAL & 40,212 & 104 & GEORGIA TECH & 9,264 \\
\hline 48 & BRITISH COLUMBIA & 38,955 & 105 & CASE WESTERN RESERVE & 8,025 \\
\hline 49 & TEMPLE & 38,720 & 106 & SUNY-STONY BROOK & 4,780 \\
\hline 50 & GEORGETOWN & 38,265 & 107 & HOWARD & 4,488 \\
\hline 51 & KANSAS & 37,475 & & ALBERTA & $\mathrm{UA} / \mathrm{NA}$ \\
\hline 52 & MINNESOTA & 37,445 & & CALIFORNIA, BERKELEY & $\mathrm{UA} / \mathrm{NA}$ \\
\hline 53 & CALIFORNIA, SAN DIEGO & 36,171 & & CORNELL & $\mathrm{UA} / \mathrm{NA}$ \\
\hline 54 & LOUISVILLE & 35,901 & & HARVARD & $\mathrm{UA} / \mathrm{NA}$ \\
\hline 55 & ARIZONA & 35,650 & & PENNSYLVANIA & $\mathrm{UA} / \mathrm{NA}$ \\
\hline 56 & CALIFORNIA, IRVINE & 35,425 & & PITTSBURGH & $\mathrm{UA} / \mathrm{NA}$ \\
\hline 57 & TEXAS TECH & 35,323 & & UTAH & $\mathrm{UA} / \mathrm{NA}$ \\
\hline
\end{tabular}


Rank Order Table 12: Expenditures for Monographs

\begin{tabular}{|c|c|c|c|c|c|}
\hline RANK & INSTITUTION & VAlue & RANK & INSTITUTION & VAlue \\
\hline 1 & YALE & $15,345,869$ & 58 & CALIFORNIA, DAVIS & $1,981,284$ \\
\hline 2 & HARVARD & $13,064,991$ & 59 & TULANE & $1,960,928$ \\
\hline 3 & PRINCETON & $8,437,800$ & 60 & WESTERN ONTARIO & $1,936,536$ \\
\hline 4 & TORONTO & $7,374,842$ & 61 & RUTGERS & $1,873,521$ \\
\hline 5 & COLUMBIA & $6,664,952$ & 62 & UTAH & $1,826,441$ \\
\hline 6 & CALIFORNIA, BERKELEY & $6,357,361$ & 63 & GEORGIA & $1,811,698$ \\
\hline 7 & CHICAGO & $6,091,102$ & 64 & BROWN & $1,710,759$ \\
\hline 8 & CORNELL & $6,059,153$ & 65 & DARTMOUTH & $1,705,162$ \\
\hline 9 & ALBERTA & $5,906,026$ & 66 & MANITOBA & $1,690,627$ \\
\hline 10 & CALIFORNIA, LOS ANGELES & $5,861,511$ & 67 & IOWA STATE & $1,684,193$ \\
\hline 11 & INDIANA & $5,757,033$ & 68 & SUNY-BUFFALO & $1,662,906$ \\
\hline 12 & MICHIGAN & $5,543,022$ & 69 & HOUSTON & $1,562,782$ \\
\hline 13 & NEW YORK & $5,209,920$ & 70 & MARYLAND & $1,539,240$ \\
\hline 14 & TEXAS A\&M & $4,801,703$ & 71 & MIT & $1,528,568$ \\
\hline 15 & NOTRE DAME & $4,489,450$ & 72 & BOSTON COLLEGE & $1,472,388$ \\
\hline 16 & DUKE & $4,320,632$ & 73 & SOUTH CAROLINA & $1,429,841$ \\
\hline 17 & PENNSYLVANIA STATE & $4,005,270$ & 74 & KENTUCKY & $1,423,984$ \\
\hline 18 & MCGILL & $3,799,947$ & 75 & NORTH CAROLINA STATE & $1,400,664$ \\
\hline 19 & PENNSYLVANIA & $3,797,236$ & 76 & FLORIDA & $1,398,497$ \\
\hline 20 & SOUTHERN CALIFORNIA & $3,782,005$ & 77 & VANDERBILT & $1,378,478$ \\
\hline 21 & WASHINGTON & $3,770,109$ & 78 & PURDUE & $1,376,389$ \\
\hline 22 & EMORY & $3,697,214$ & 79 & SYRACUSE & $1,353,362$ \\
\hline 23 & BRITISH COLUMBIA & $3,648,620$ & 80 & NEW MEXICO & $1,348,879$ \\
\hline 24 & NORTHWESTERN & $3,607,610$ & 81 & ILLINOIS, CHICAGO & $1,334,871$ \\
\hline 25 & OKLAHOMA & $3,326,015$ & 82 & VIRGINIA TECH & $1,204,203$ \\
\hline 26 & RICE & $3,223,688$ & 83 & ROCHESTER & $1,193,180$ \\
\hline 27 & CALGARY & $3,031,863$ & 84 & MCMASTER & $1,183,460$ \\
\hline 28 & NORTH CAROLINA & $3,030,479$ & 85 & ARIZONA STATE & $1,176,474$ \\
\hline 29 & CALIFORNIA, SAN DIEGO & $3,015,924$ & 86 & QUEEN'S & $1,167,682$ \\
\hline 30 & MINNESOTA & $3,006,117$ & 87 & GUELPH & $1,160,317$ \\
\hline 31 & VIRGINIA & $2,980,240$ & 88 & OREGON & $1,159,041$ \\
\hline 32 & PITTSBURGH & $2,923,074$ & 89 & HAWAII & $1,145,314$ \\
\hline 33 & ILLINOIS, URBANA & $2,839,435$ & 90 & CONNECTICUT & $1,117,334$ \\
\hline 34 & MONTREAL & $2,641,805$ & 91 & ALABAMA & $1,114,921$ \\
\hline 35 & TEXAS & $2,627,065$ & 92 & COLORADO STATE & $1,055,691$ \\
\hline 36 & JOHNS HOPKINS & $2,582,724$ & 93 & CALIFORNIA, SANTA BARBARA & 989,473 \\
\hline 37 & OHIO STATE & $2,511,656$ & 94 & MISSOURI & 967,024 \\
\hline 38 & BRIGHAM YOUNG & $2,505,763$ & 95 & WASHINGTON STATE & 952,370 \\
\hline 39 & IOWA & $2,504,908$ & 96 & LOUISIANA STATE & 940,843 \\
\hline 40 & GEORGETOWN & $2,494,661$ & 97 & FLORIDA STATE & 934,853 \\
\hline 41 & GEORGE WASHINGTON & $2,469,176$ & 98 & $\mathrm{OHIO}$ & 919,119 \\
\hline 42 & ARIZONA & $2,420,279$ & 99 & BOSTON & 894,004 \\
\hline 43 & MICHIGAN STATE & $2,385,685$ & 100 & LOUISVILLE & 876,426 \\
\hline 44 & TENNESSEE & $2,346,711$ & 101 & MASSACHUSETTS & 796,144 \\
\hline 45 & TEMPLE & $2,336,860$ & 102 & KENT STATE & 758,152 \\
\hline 46 & TEXAS TECH & $2,320,596$ & 103 & WATERLOO & 747,903 \\
\hline 47 & DELAWARE & $2,302,481$ & 104 & SOUTHERN ILLINOIS & 711,745 \\
\hline 48 & MIAMI & $2,283,175$ & 105 & CASE WESTERN RESERVE & 694,410 \\
\hline 49 & CINCINNATI & $2,275,243$ & 106 & WAYNE STATE & 620,886 \\
\hline 50 & YORK & $2,244,864$ & 107 & NEBRASKA & 585,693 \\
\hline 51 & COLORADO & $2,216,558$ & 108 & CALIFORNIA, RIVERSIDE & 556,349 \\
\hline 52 & LAVAL & $2,189,590$ & 109 & SUNY-ALBANY & 549,758 \\
\hline 53 & CALIFORNIA, IRVINE & $2,152,745$ & 110 & AUBURN & 518,171 \\
\hline 54 & SASKATCHEWAN & $2,146,004$ & 111 & SUNY-STONY BROOK & 301,426 \\
\hline 55 & WASHINGTON U.-ST. LOUIS & $2,135,058$ & 112 & GEORGIA TECH & 276,824 \\
\hline 56 & WISCONSIN & $2,111,462$ & 113 & HOWARD & 244,960 \\
\hline 57 & KANSAS & $2,056,586$ & & OKLAHOMA STATE & $\mathrm{UA} / \mathrm{NA}$ \\
\hline
\end{tabular}


Rank Order Table 13: Current Serials Purchased (Titles)

\begin{tabular}{|c|c|c|c|c|c|}
\hline RANK & INSTITUTION & VALUE & RANK & INSTITUTION & VALUE \\
\hline 1 & COLUMBIA & 115,050 & & QUEEN'S & 52,667 \\
\hline 2 & ILLINOIS, URBANA & 108,089 & 59 & ALABAMA & 51,665 \\
\hline 3 & ALBERTA & 101,539 & 60 & NORTH CAROLINA & 51,091 \\
\hline 4 & LOUISIANA STATE & 97,524 & 61 & CONNECTICUT & 50,436 \\
\hline 5 & YALE & 86,846 & 62 & MCGILL & 49,877 \\
\hline 6 & CINCINNATI & 86,563 & 63 & FLORIDA & 49,186 \\
\hline 7 & LOUISVILLE & 86,077 & 64 & HOUSTON & 49,181 \\
\hline 8 & MONTREAL & 82,785 & 65 & BOSTON & 49,030 \\
\hline 9 & BRITISH COLUMBIA & 82,202 & 66 & BRIGHAM YOUNG & 48,793 \\
\hline 10 & SUNY-STONY BROOK & 80,421 & 67 & GUELPH & 46,691 \\
\hline 11 & ARIZONA & 79,985 & 68 & LAVAL & 46,196 \\
\hline 12 & OHIO STATE & 79,751 & 69 & TEMPLE & 46,070 \\
\hline 13 & PITTSBURGH & 78,691 & 70 & BROWN & 45,669 \\
\hline 14 & ARIZONA STATE & 78,420 & 71 & WAYNE STATE & 44,239 \\
\hline 15 & SOUTHERN CALIFORNIA & 76,335 & 72 & KENTUCKY & 43,730 \\
\hline 16 & NEW YORK & 75,188 & 73 & RUTGERS & 42,783 \\
\hline 17 & MICHIGAN STATE & 74,459 & 74 & HAWAII & 42,276 \\
\hline 18 & TORONTO & 73,909 & 75 & MIT & 41,996 \\
\hline 19 & CALIFORNIA, BERKELEY & 72,944 & 76 & WASHINGTON & 41,990 \\
\hline 20 & INDIANA & 72,176 & 77 & ILLINOIS, CHICAGO & 40,100 \\
\hline 21 & MIAMI & 71,223 & 78 & COLORADO & 39,978 \\
\hline 22 & MINNESOTA & 70,597 & 79 & TENNESSEE & 39,913 \\
\hline 23 & WISCONSIN & 69,757 & 80 & MCMASTER & 39,726 \\
\hline 24 & NORTHWESTERN & 68,751 & 81 & MASSACHUSETTS & 37,277 \\
\hline 25 & PENNSYLVANIA STATE & 68,556 & 82 & SOUTHERN ILLINOIS & 36,938 \\
\hline 26 & TEXAS & 68,073 & 83 & SUNY-ALBANY & 36,345 \\
\hline 27 & YORK & 67,266 & 84 & CALGARY & 36,040 \\
\hline 28 & GEORGIA & 66,472 & 85 & CALIFORNIA, IRVINE & 35,957 \\
\hline 29 & NOTRE DAME & 66,017 & 86 & CALIFORNIA, SANTA BARBARA & 35,259 \\
\hline 30 & CASE WESTERN RESERVE & 65,734 & 87 & PURDUE & 34,785 \\
\hline 31 & IOWA STATE & 64,626 & 88 & NEBRASKA & 33,000 \\
\hline 32 & EMORY & 64,321 & 89 & NORTH CAROLINA STATE & 32,369 \\
\hline 33 & GEORGE WASHINGTON & 64,311 & 90 & UTAH & 31,280 \\
\hline 34 & PENNSYLVANIA & 62,731 & 91 & VANDERBILT & 30,837 \\
\hline 35 & WESTERN ONTARIO & 62,260 & 92 & COLORADO STATE & 29,664 \\
\hline 36 & GEORGETOWN & 62,196 & 93 & OHIO & 29,544 \\
\hline 37 & CALIFORNIA, RIVERSIDE & 62,029 & 94 & ROCHESTER & 28,810 \\
\hline 38 & RICE & 61,821 & 95 & SASKATCHEWAN & 28,223 \\
\hline 39 & JOHNS HOPKINS & 61,598 & 96 & SYRACUSE & 27,637 \\
\hline 40 & CALIFORNIA, SAN DIEGO & 61,211 & 97 & MANITOBA & 26,021 \\
\hline 41 & SUNY-BUFFALO & 60,526 & 98 & MISSOURI & 24,857 \\
\hline 42 & TEXAS A\&M & 60,339 & 99 & CALIFORNIA, LOS ANGELES & 23,504 \\
\hline 43 & TEXAS TECH & 59,895 & 100 & OREGON & 23,421 \\
\hline 44 & CHICAGO & 59,714 & 101 & BOSTON COLLEGE & 23,129 \\
\hline 45 & NEW MEXICO & 58,917 & 102 & MARYLAND & 23,039 \\
\hline 46 & DARTMOUTH & 58,203 & 103 & DELAWARE & 21,685 \\
\hline 47 & PRINCETON & 58,045 & 104 & AUBURN & 19,437 \\
\hline 48 & KENT STATE & 57,817 & 105 & WATERLOO & 16,138 \\
\hline 49 & OKLAHOMA & 57,803 & 106 & HOWARD & 15,204 \\
\hline 50 & KANSAS & 57,674 & 107 & SOUTH CAROLINA & 14,384 \\
\hline 51 & DUKE & 57,365 & 108 & VIRGINIA TECH & 13,360 \\
\hline 52 & FLORIDA STATE & 56,471 & 109 & GEORGIA TECH & 10,754 \\
\hline 53 & OKLAHOMA STATE & 55,672 & & CORNELL & $\mathrm{UA} / \mathrm{NA}$ \\
\hline 54 & WASHINGTON U.-ST. LOUIS & 55,097 & & HARVARD & $\mathrm{UA} / \mathrm{NA}$ \\
\hline 55 & IOWA & 55,018 & & MICHIGAN & $\mathrm{UA} / \mathrm{NA}$ \\
\hline 56 & TULANE & 54,864 & & VIRGINIA & $\mathrm{UA} / \mathrm{NA}$ \\
\hline 57 & CALIFORNIA, DAVIS & 53,466 & & WASHINGTON STATE & $\mathrm{UA} / \mathrm{NA}$ \\
\hline
\end{tabular}


Rank Order Table 14: Expenditures for Current Serials

\begin{tabular}{|c|c|c|c|c|c|}
\hline RANK & INSTITUTION & Value & RANK & INSTITUTION & VALUE \\
\hline 1 & COLUMBIA & $15,015,672$ & 58 & MIT & $7,176,222$ \\
\hline 2 & MICHIGAN & $14,141,584$ & 59 & GEORGE WASHINGTON & $7,101,214$ \\
\hline 3 & MINNESOTA & $12,871,946$ & 60 & CALIFORNIA, IRVINE & $7,084,869$ \\
\hline 4 & PENNSYLVANIA STATE & $12,760,610$ & 61 & SASKATCHEWAN & $7,007,379$ \\
\hline 5 & TEXAS A\&M & $12,305,942$ & 62 & LAVAL & $6,990,673$ \\
\hline 6 & WASHINGTON & $12,131,204$ & 63 & ROCHESTER & $6,984,764$ \\
\hline 7 & CHICAGO & $11,982,017$ & 64 & BRIGHAM YOUNG & $6,890,140$ \\
\hline 8 & MIAMI & $11,176,016$ & 65 & KENTUCKY & $6,827,718$ \\
\hline 9 & BRITISH COLUMBIA & $11,092,400$ & 66 & NORTH CAROLINA & $6,810,130$ \\
\hline 10 & TORONTO & $10,802,507$ & 67 & HAWAII & $6,791,920$ \\
\hline 11 & IOWA & $10,782,240$ & 68 & CALIFORNIA, SAN DIEGO & $6,764,591$ \\
\hline 12 & ILLINOIS, URBANA & $10,773,374$ & 69 & ALABAMA & $6,764,436$ \\
\hline 13 & PRINCETON & $10,709,460$ & 70 & EMORY & $6,694,006$ \\
\hline 14 & CALIFORNIA, BERKELEY & $10,540,298$ & 71 & SUNY-BUFFALO & $6,692,869$ \\
\hline 15 & DUKE & $10,453,502$ & 72 & KANSAS & $6,660,380$ \\
\hline 16 & IOWA STATE & $10,215,830$ & 73 & HOUSTON & $6,633,041$ \\
\hline 17 & PENNSYLVANIA & $10,215,771$ & 74 & CALIFORNIA, DAVIS & $6,560,659$ \\
\hline 18 & TEXAS & $10,204,174$ & 75 & QUEEN'S & $6,500,290$ \\
\hline 19 & NEW YORK & $10,111,441$ & 76 & TULANE & $6,463,567$ \\
\hline 20 & ALBERTA & $10,050,328$ & 77 & RICE & $6,443,468$ \\
\hline 21 & VANDERBILT & $9,576,572$ & 78 & SOUTH CAROLINA & $6,377,527$ \\
\hline 22 & PITTSBURGH & $9,523,942$ & 79 & WISCONSIN & $6,351,064$ \\
\hline 23 & MONTREAL & $9,427,747$ & 80 & TEMPLE & $6,337,644$ \\
\hline 24 & FLORIDA & $9,380,631$ & 81 & SUNY-STONY BROOK & $6,094,482$ \\
\hline 25 & NORTHWESTERN & $9,014,225$ & 82 & SOUTHERN ILLINOIS & $6,012,763$ \\
\hline 26 & ARIZONA & $8,980,989$ & 83 & FLORIDA STATE & $6,007,992$ \\
\hline 27 & ARIZONA STATE & $8,962,161$ & 84 & BROWN & $5,936,815$ \\
\hline 28 & SOUTHERN CALIFORNIA & $8,888,694$ & 85 & NEW MEXICO & $5,911,858$ \\
\hline 29 & TENNESSEE & $8,805,394$ & 86 & DELAWARE & $5,896,667$ \\
\hline 30 & OHIO STATE & $8,789,376$ & 87 & CASE WESTERN RESERVE & $5,831,618$ \\
\hline 31 & MICHIGAN STATE & $8,778,753$ & 88 & NEBRASKA & $5,689,064$ \\
\hline 32 & OKLAHOMA & $8,702,979$ & 89 & YALE & $5,670,704$ \\
\hline 33 & WASHINGTON U.-ST. LOUIS & $8,619,649$ & 90 & MCMASTER & $5,666,674$ \\
\hline 34 & RUTGERS & $8,501,656$ & 91 & SYRACUSE & $5,666,523$ \\
\hline 35 & CORNELL & $8,472,429$ & 92 & MANITOBA & $5,638,030$ \\
\hline 36 & HARVARD & $8,448,811$ & 93 & MARYLAND & $5,624,692$ \\
\hline 37 & INDIANA & $8,278,469$ & 94 & UTAH & $5,349,353$ \\
\hline 38 & BOSTON & $8,275,790$ & 95 & VIRGINIA TECH & $5,335,345$ \\
\hline 39 & MCGILL & $8,269,481$ & 96 & LOUISIANA STATE & $5,308,904$ \\
\hline 40 & WESTERN ONTARIO & $8,248,564$ & 97 & BOSTON COLLEGE & $5,231,355$ \\
\hline 41 & TEXAS TECH & $8,238,684$ & 98 & MASSACHUSETTS & $5,226,388$ \\
\hline 42 & CALIFORNIA, LOS ANGELES & $8,099,419$ & 99 & AUBURN & $5,186,086$ \\
\hline 43 & GEORGETOWN & $8,009,661$ & 100 & COLORADO STATE & $5,072,641$ \\
\hline 44 & WAYNE STATE & $7,990,111$ & 101 & WASHINGTON STATE & $4,985,493$ \\
\hline 45 & GEORGIA & $7,969,205$ & 102 & WATERLOO & $4,774,658$ \\
\hline 46 & PURDUE & $7,921,059$ & 103 & GEORGIA TECH & $4,571,221$ \\
\hline 47 & CONNECTICUT & $7,910,971$ & 104 & ILLINOIS, CHICAGO & $4,398,769$ \\
\hline 48 & VIRGINIA & $7,821,461$ & 105 & SUNY-ALBANY & $4,362,545$ \\
\hline 49 & DARTMOUTH & $7,567,386$ & 106 & LOUISVILLE & $4,282,651$ \\
\hline 50 & COLORADO & $7,430,771$ & 107 & CALIFORNIA, RIVERSIDE & $4,230,726$ \\
\hline 51 & CALGARY & $7,402,153$ & 108 & CALIFORNIA, SANTA BARBARA & $4,217,070$ \\
\hline 52 & JOHNS HOPKINS & $7,368,569$ & 109 & OREGON & $4,085,978$ \\
\hline 53 & YORK & $7,359,970$ & 110 & OHIO & $4,032,388$ \\
\hline 54 & NORTH CAROLINA STATE & $7,268,906$ & 111 & GUELPH & $3,681,947$ \\
\hline 55 & MISSOURI & $7,248,586$ & 112 & HOWARD & $3,658,418$ \\
\hline 56 & NOTRE DAME & $7,194,446$ & 113 & KENT STATE & $3,445,792$ \\
\hline 57 & CINCINNATI & $7,192,136$ & & OKLAHOMA STATE & $\mathrm{UA} / \mathrm{NA}$ \\
\hline
\end{tabular}


Rank Order Table 15: Total Items Loaned (ILL/DD)

\begin{tabular}{|c|c|c|c|c|c|}
\hline RANK & INSTITUTION & Value & RANK & INSTITUTION & VALUE \\
\hline 1 & MINNESOTA & 178,255 & 58 & GEORGETOWN & 35,259 \\
\hline 2 & OHIO STATE & 148,084 & 59 & TENNESSEE & 34,534 \\
\hline 3 & WASHINGTON & 95,895 & 60 & MIAMI & 34,202 \\
\hline 4 & $\mathrm{OHIO}$ & 91,863 & 61 & IOWA STATE & 34,033 \\
\hline 5 & ILLINOIS, URBANA & 91,700 & 62 & CASE WESTERN RESERVE & 33,485 \\
\hline 6 & WISCONSIN & 90,581 & 63 & NEBRASKA & 32,166 \\
\hline 7 & COLORADO STATE & 81,331 & 64 & GEORGE WASHINGTON & 31,907 \\
\hline 8 & PITTSBURGH & 76,898 & 65 & CALIFORNIA, SAN DIEGO & 31,545 \\
\hline 9 & PENNSYLVANIA STATE & 75,432 & 66 & OKLAHOMA STATE & 31,401 \\
\hline 10 & PENNSYLVANIA & 70,875 & 67 & KENTUCKY & 30,301 \\
\hline 11 & MICHIGAN STATE & 68,911 & 68 & TORONTO & 30,099 \\
\hline 12 & OREGON & 67,678 & 69 & TEMPLE & 29,292 \\
\hline 13 & NORTH CAROLINA & 66,072 & 70 & PURDUE & 28,638 \\
\hline 14 & ILLINOIS, CHICAGO & 64,997 & 71 & MONTREAL & 27,126 \\
\hline 15 & BRIGHAM YOUNG & 62,169 & 72 & SUNY-BUFFALO & 26,996 \\
\hline 16 & TEXAS & 61,799 & 73 & BOSTON COLLEGE & 26,599 \\
\hline 17 & WASHINGTON U.-ST. LOUIS & 59,715 & 74 & CALIFORNIA, IRVINE & 26,281 \\
\hline 18 & CINCINNATI & 59,462 & 75 & VANDERBILT & 25,748 \\
\hline 19 & IOWA & 59,427 & 76 & NOTRE DAME & 25,342 \\
\hline 20 & MISSOURI & 59,060 & 77 & MARYLAND & 25,268 \\
\hline 21 & CORNELL & 58,351 & 78 & NEW YORK & 25,135 \\
\hline 22 & ARIZONA & 58,338 & 79 & NEW MEXICO & 25,031 \\
\hline 23 & CHICAGO & 56,493 & 80 & LOUISVILLE & 24,753 \\
\hline 24 & COLORADO & 55,834 & 81 & HOUSTON & 24,690 \\
\hline 25 & MICHIGAN & 52,862 & 82 & SOUTHERN CALIFORNIA & 24,473 \\
\hline 26 & ARIZONA STATE & 51,378 & 83 & BRITISH COLUMBIA & 23,978 \\
\hline 27 & SOUTHERN ILLINOIS & 51,209 & 84 & FLORIDA STATE & 23,607 \\
\hline 28 & WATERLOO & 50,116 & 85 & CALIFORNIA, SANTA BARBARA & 23,207 \\
\hline 29 & KANSAS & 49,923 & 86 & ROCHESTER & 22,571 \\
\hline 30 & ALBERTA & 49,147 & 87 & SUNY-STONY BROOK & 21,195 \\
\hline 31 & WAYNE STATE & 48,424 & 88 & MCGILL & 20,387 \\
\hline 32 & PRINCETON & 47,441 & 89 & VIRGINIA TECH & 20,010 \\
\hline 33 & CALIFORNIA, LOS ANGELES & 46,796 & 90 & BOSTON & 19,710 \\
\hline 34 & YALE & 46,497 & 91 & NORTH CAROLINA STATE & 19,495 \\
\hline 35 & COLUMBIA & 46,134 & 92 & CALGARY & 19,016 \\
\hline 36 & TEXAS A\&M & 45,649 & 93 & MANITOBA & 18,981 \\
\hline 37 & INDIANA & 45,546 & 94 & LAVAL & 18,724 \\
\hline 38 & JOHNS HOPKINS & 45,350 & 95 & DELAWARE & 18,700 \\
\hline 39 & RUTGERS & 45,230 & 96 & FLORIDA & 18,238 \\
\hline 40 & GUELPH & 44,136 & 97 & MIT & 18,086 \\
\hline 41 & UTAH & 42,479 & 98 & QUEEN'S & 17,610 \\
\hline 42 & OKLAHOMA & 42,185 & 99 & SOUTH CAROLINA & 17,142 \\
\hline 43 & DARTMOUTH & 41,775 & 100 & LOUISIANA STATE & 16,593 \\
\hline 44 & CALIFORNIA, DAVIS & 41,383 & 101 & SASKATCHEWAN & 16,113 \\
\hline 45 & GEORGIA & 40,706 & 102 & MCMASTER & 15,394 \\
\hline 46 & CONNECTICUT & 40,370 & 103 & YORK & 15,335 \\
\hline 47 & HARVARD & 39,760 & 104 & TULANE & 14,929 \\
\hline 48 & MASSACHUSETTS & 37,532 & 105 & KENT STATE & 14,062 \\
\hline 49 & NORTHWESTERN & 37,518 & 106 & AUBURN & 13,542 \\
\hline 50 & CALIFORNIA, BERKELEY & 37,362 & 107 & RICE & 13,437 \\
\hline 51 & BROWN & 37,189 & 108 & WESTERN ONTARIO & 13,138 \\
\hline 52 & VIRGINIA & 37,176 & 109 & GEORGIA TECH & 12,694 \\
\hline 53 & EMORY & 36,890 & 110 & SUNY-ALBANY & 12,428 \\
\hline 54 & CALIFORNIA, RIVERSIDE & 36,452 & 111 & SYRACUSE & 12,427 \\
\hline 55 & WASHINGTON STATE & 36,420 & 112 & ALABAMA & 11,983 \\
\hline 56 & TEXAS TECH & 35,890 & 113 & HOWARD & 7,872 \\
\hline 57 & DUKE & 35,589 & 114 & HAWAII & 6,843 \\
\hline
\end{tabular}


Rank Order Table 16: Total Items Borrowed (ILL/DD)

\begin{tabular}{|c|c|c|c|c|c|}
\hline RANK & INSTITUTION & VAlue & RANK & INSTITUTION & VALUE \\
\hline 1 & WASHINGTON & 97,626 & 58 & CALIFORNIA, IRVINE & 28,187 \\
\hline 2 & OHIO STATE & 96,431 & 59 & PITTSBURGH & 28,180 \\
\hline 3 & WISCONSIN & 95,513 & 60 & CALIFORNIA, SAN DIEGO & 27,976 \\
\hline 4 & PENNSYLVANIA & 93,481 & 61 & CALGARY & 27,506 \\
\hline 5 & ILLINOIS, URBANA & 78,564 & 62 & NORTH CAROLINA STATE & 27,229 \\
\hline 6 & OHIO & 74,464 & 63 & TEMPLE & 26,406 \\
\hline 7 & COLORADO STATE & 65,209 & 64 & CALIFORNIA, BERKELEY & 26,202 \\
\hline 8 & OREGON & 58,336 & 65 & KENTUCKY & 26,164 \\
\hline 9 & GEORGE WASHINGTON & 57,876 & 66 & CALIFORNIA, RIVERSIDE & 25,623 \\
\hline 10 & COLUMBIA & 55,437 & 67 & ROCHESTER & 25,064 \\
\hline 11 & PENNSYLVANIA STATE & 54,445 & 68 & NOTRE DAME & 24,898 \\
\hline 12 & ILLINOIS, CHICAGO & 53,004 & 69 & MANITOBA & 24,583 \\
\hline 13 & TEXAS A\&M & 52,687 & 70 & DELAWARE & 24,559 \\
\hline 14 & MICHIGAN & 52,435 & 71 & CALIFORNIA, DAVIS & 24,345 \\
\hline 15 & WASHINGTON U.-ST. LOUIS & 51,249 & 72 & OKLAHOMA STATE & 23,836 \\
\hline 16 & CONNECTICUT & 48,491 & 73 & SUNY-BUFFALO & 23,676 \\
\hline 17 & INDIANA & 46,885 & 74 & WASHINGTON STATE & 23,105 \\
\hline 18 & GUELPH & 46,607 & 75 & SUNY-ALBANY & 22,371 \\
\hline 19 & WATERLOO & 46,099 & 76 & TENNESSEE & 21,944 \\
\hline 20 & ARIZONA & 45,608 & 77 & SYRACUSE & 21,452 \\
\hline 21 & PURDUE & 44,428 & 78 & BOSTON & 21,321 \\
\hline 22 & ARIZONA STATE & 44,348 & 79 & VIRGINIA TECH & 20,705 \\
\hline 23 & MASSACHUSETTS & 44,014 & 80 & SOUTHERN CALIFORNIA & 20,439 \\
\hline 24 & MISSOURI & 43,809 & 81 & MIT & 20,096 \\
\hline 25 & JOHNS HOPKINS & 43,247 & 82 & SASKATCHEWAN & 19,847 \\
\hline 26 & WAYNE STATE & 42,671 & 83 & MONTREAL & 19,824 \\
\hline 27 & YALE & 42,194 & 84 & BOSTON COLLEGE & 19,577 \\
\hline 28 & MINNESOTA & 41,710 & 85 & NORTH CAROLINA & 19,011 \\
\hline 29 & SOUTHERN ILLINOIS & 41,563 & 86 & CHICAGO & 18,824 \\
\hline 30 & CINCINNATI & 40,755 & 87 & EMORY & 18,776 \\
\hline 31 & NEW YORK & 39,993 & 88 & IOWA STATE & 17,754 \\
\hline 32 & BROWN & 39,355 & 89 & ALBERTA & 17,670 \\
\hline 33 & KANSAS & 39,196 & 90 & SOUTH CAROLINA & 17,391 \\
\hline 34 & MARYLAND & 39,117 & 91 & HOUSTON & 16,771 \\
\hline 35 & CALIFORNIA, LOS ANGELES & 37,922 & 92 & BRITISH COLUMBIA & 16,602 \\
\hline 36 & GEORGETOWN & 37,418 & 93 & VANDERBILT & 16,505 \\
\hline 37 & UTAH & 37,036 & 94 & LOUISIANA STATE & 16,231 \\
\hline 38 & CALIFORNIA, SANTA BARBARA & 36,918 & 95 & FLORIDA & 15,899 \\
\hline 39 & PRINCETON & 35,857 & 96 & HARVARD & 14,594 \\
\hline 40 & RUTGERS & 35,774 & 97 & MCMASTER & 14,519 \\
\hline 41 & CASE WESTERN RESERVE & 35,459 & 98 & MCGILL & 14,108 \\
\hline 42 & NORTHWESTERN & 34,168 & 99 & FLORIDA STATE & 13,854 \\
\hline 43 & MICHIGAN STATE & 33,983 & 100 & MIAMI & 13,807 \\
\hline 44 & CORNELL & 33,826 & 101 & GEORGIA TECH & 12,305 \\
\hline 45 & IOWA & 33,684 & 102 & SUNY-STONY BROOK & 11,824 \\
\hline 46 & COLORADO & 32,948 & 103 & ALABAMA & 11,523 \\
\hline 47 & OKLAHOMA & 32,889 & 104 & TULANE & 11,192 \\
\hline 48 & NEW MEXICO & 32,666 & 105 & RICE & 10,841 \\
\hline 49 & BRIGHAM YOUNG & 32,332 & 106 & AUBURN & 10,265 \\
\hline 50 & VIRGINIA & 32,075 & 107 & WESTERN ONTARIO & 9,613 \\
\hline 51 & TEXAS TECH & 32,012 & 108 & LAVAL & 9,154 \\
\hline 52 & DARTMOUTH & 31,634 & 109 & TORONTO & 9,119 \\
\hline 53 & NEBRASKA & 31,139 & 110 & KENT STATE & 8,579 \\
\hline 54 & HAWAII & 30,273 & 111 & GEORGIA & 7,861 \\
\hline 55 & DUKE & 29,378 & 112 & QUEEN'S & 7,168 \\
\hline 56 & LOUISVILLE & 28,640 & 113 & YORK & 5,554 \\
\hline 57 & TEXAS & 28,329 & 114 & HOWARD & 4,764 \\
\hline
\end{tabular}

Rank Order Tables of University Libraries · 89 
Rank Order Table 17: Professional Staff (FTE)

\begin{tabular}{|c|c|c|c|c|c|}
\hline RANK & INSTITUTION & VALUE & RANK & INSTITUTION & VALUE \\
\hline 1 & HARVARD & 565 & 58 & MIAMI & 83 \\
\hline 2 & COLUMBIA & 311 & 59 & NOTRE DAME & 82 \\
\hline 3 & YALE & 266 & 60 & GEORGIA & 79 \\
\hline 4 & WISCONSIN & 245 & 61 & CINCINNATI & 78 \\
\hline 5 & CALIFORNIA, BERKELEY & 243 & 61 & MICHIGAN STATE & 78 \\
\hline 6 & WASHINGTON & 194 & 63 & BROWN & 77 \\
\hline 7 & ILLINOIS, URBANA & 191 & 63 & MCGILL & 77 \\
\hline 8 & DUKE & 183 & 65 & BOSTON COLLEGE & 75 \\
\hline 9 & OHIO STATE & 172 & 65 & TEMPLE & 75 \\
\hline 10 & TORONTO & 171 & 67 & ALABAMA & 73 \\
\hline 11 & MICHIGAN & 167 & 68 & OREGON & 72 \\
\hline 12 & CALIFORNIA, LOS ANGELES & 163 & 68 & SOUTH CAROLINA & 72 \\
\hline 13 & PENNSYLVANIA STATE & 161 & 68 & SUNY-ALBANY & 72 \\
\hline 14 & NORTH CAROLINA & 158 & 71 & CALGARY & 71 \\
\hline 15 & TEXAS & 149 & 72 & CHICAGO & 70 \\
\hline 16 & NEW YORK & 148 & 72 & WESTERN ONTARIO & 70 \\
\hline 17 & TEXAS A\&M & 147 & 74 & CALIFORNIA, IRVINE & 69 \\
\hline 18 & PRINCETON & 143 & 75 & OKLAHOMA STATE & 66 \\
\hline 19 & PENNSYLVANIA & 136 & 76 & ILLINOIS, CHICAGO & 65 \\
\hline 20 & INDIANA & 135 & 77 & PURDUE & 64 \\
\hline 21 & NORTH CAROLINA STATE & 129 & 78 & MANITOBA & 63 \\
\hline 21 & SOUTHERN CALIFORNIA & 129 & 79 & FLORIDA STATE & 62 \\
\hline 23 & BRIGHAM YOUNG & 126 & 79 & SYRACUSE & 62 \\
\hline 24 & JOHNS HOPKINS & 124 & 79 & YORK & 62 \\
\hline 25 & BRITISH COLUMBIA & 123 & 82 & HOUSTON & 61 \\
\hline 26 & MARYLAND & 121 & 82 & LAVAL & 61 \\
\hline 26 & NORTHWESTERN & 121 & 82 & OKLAHOMA & 61 \\
\hline 26 & PITTSBURGH & 121 & 85 & NEW MEXICO & 60 \\
\hline 29 & CORNELL & 118 & 86 & CASE WESTERN RESERVE & 59 \\
\hline 30 & MONTREAL & 114 & 86 & MASSACHUSETTS & 59 \\
\hline 31 & MINNESOTA & 112 & 86 & RICE & 59 \\
\hline 32 & CALIFORNIA, SAN DIEGO & 110 & 89 & DELAWARE & 58 \\
\hline 33 & VANDERBILT & 108 & 90 & MCMASTER & 57 \\
\hline 34 & BOSTON & 104 & 91 & COLORADO & 56 \\
\hline 34 & IOWA & 104 & 91 & MISSOURI & 56 \\
\hline 34 & VIRGINIA & 104 & 93 & CALIFORNIA, DAVIS & 55 \\
\hline 37 & KANSAS & 103 & 93 & DARTMOUTH & 55 \\
\hline 37 & TEXAS TECH & 103 & 93 & KENT STATE & 55 \\
\hline 39 & SUNY-BUFFALO & 102 & 96 & NEBRASKA & 53 \\
\hline 40 & ROCHESTER & 101 & 97 & IOWA STATE & 52 \\
\hline 40 & WAYNE STATE & 101 & 97 & SOUTHERN ILLINOIS & 52 \\
\hline 42 & GEORGETOWN & 99 & 97 & WASHINGTON STATE & 52 \\
\hline 43 & FLORIDA & 98 & 100 & GUELPH & 51 \\
\hline 44 & MIT & 96 & 100 & LOUISIANA STATE & 51 \\
\hline 45 & EMORY & 93 & 100 & LOUISVILLE & 51 \\
\hline 46 & ARIZONA & 91 & 100 & SASKATCHEWAN & 51 \\
\hline 46 & ARIZONA STATE & 91 & 104 & COLORADO STATE & 48 \\
\hline 46 & WASHINGTON U.-ST. LOUIS & 91 & 104 & $\mathrm{OHIO}$ & 48 \\
\hline 49 & HAWAII & 90 & 106 & CALIFORNIA, SANTA BARBARA & 47 \\
\hline 49 & RUTGERS & 90 & 107 & GEORGIA TECH & 46 \\
\hline 51 & ALBERTA & 88 & 108 & AUBURN & 45 \\
\hline 52 & KENTUCKY & 87 & 108 & CALIFORNIA, RIVERSIDE & 45 \\
\hline 52 & TENNESSEE & 87 & 108 & TULANE & 45 \\
\hline 54 & SUNY-STONY BROOK & 86 & 111 & QUEEN'S & 44 \\
\hline 55 & CONNECTICUT & 85 & 112 & HOWARD & 38 \\
\hline 55 & GEORGE WASHINGTON & 85 & 112 & VIRGINIA TECH & 38 \\
\hline 57 & UTAH & 84 & 112 & WATERLOO & 38 \\
\hline
\end{tabular}


Rank Order Table 18: Support Staff (FTE)

\begin{tabular}{|c|c|c|c|c|c|}
\hline RANK & INSTITUTION & VAlue & RANK & INSTITUTION & VALUE \\
\hline 1 & HARVARD & 529 & 58 & KANSAS & 113 \\
\hline 2 & YALE & 378 & 58 & DARTMOUTH & 113 \\
\hline 3 & PENNSYLVANIA STATE & 370 & 60 & YORK & 110 \\
\hline 4 & TORONTO & 343 & 61 & GEORGE WASHINGTON & 108 \\
\hline 5 & MICHIGAN & 309 & 61 & QUEEN'S & 108 \\
\hline 6 & TEXAS & 289 & 63 & WESTERN ONTARIO & 106 \\
\hline 7 & CORNELL & 286 & 64 & NORTH CAROLINA STATE & 105 \\
\hline 8 & CALIFORNIA, LOS ANGELES & 272 & 65 & SOUTH CAROLINA & 103 \\
\hline 9 & MONTREAL & 248 & 66 & VANDERBILT & 102 \\
\hline 10 & RUTGERS & 235 & 66 & PURDUE & 102 \\
\hline 11 & NEW YORK & 226 & 66 & NEBRASKA & 102 \\
\hline 12 & COLUMBIA & 225 & 69 & MISSOURI & 101 \\
\hline 13 & PRINCETON & 215 & 70 & BROWN & 99 \\
\hline 14 & NORTH CAROLINA & 214 & 71 & FLORIDA STATE & 98 \\
\hline 14 & ALBERTA & 214 & 72 & MARYLAND & 96 \\
\hline 16 & VIRGINIA & 206 & 72 & BOSTON & 96 \\
\hline 17 & MINNESOTA & 203 & 72 & OKLAHOMA & 96 \\
\hline 18 & CALIFORNIA, BERKELEY & 201 & 75 & SYRACUSE & 95 \\
\hline 19 & ILLINOIS, URBANA & 200 & 76 & DELAWARE & 94 \\
\hline 20 & WASHINGTON & 199 & 77 & ILLINOIS, CHICAGO & 93 \\
\hline 21 & CALIFORNIA, SAN DIEGO & 188 & 77 & LOUISVILLE & 93 \\
\hline 22 & CHICAGO & 181 & 79 & MIT & 92 \\
\hline 23 & FLORIDA & 180 & 79 & TEMPLE & 92 \\
\hline 24 & BRITISH COLUMBIA & 178 & 81 & SOUTHERN ILLINOIS & 91 \\
\hline 25 & PENNSYLVANIA & 176 & 81 & WATERLOO & 91 \\
\hline 26 & PITTSBURGH & 171 & 83 & SASKATCHEWAN & 88 \\
\hline 26 & ARIZONA STATE & 171 & 84 & IOWA STATE & 85 \\
\hline 26 & GEORGIA & 171 & 85 & TULANE & 84 \\
\hline 29 & CALGARY & 168 & 86 & WASHINGTON STATE & 83 \\
\hline 30 & WISCONSIN & 165 & 86 & VIRGINIA TECH & 83 \\
\hline 30 & INDIANA & 165 & 88 & OREGON & 82 \\
\hline 32 & UTAH & 157 & 89 & WAYNE STATE & 81 \\
\hline 33 & EMORY & 155 & 89 & HAWAII & 81 \\
\hline 34 & LAVAL & 154 & 89 & MCMASTER & 81 \\
\hline 35 & OHIO STATE & 147 & 89 & CALIFORNIA, RIVERSIDE & 81 \\
\hline 35 & MCGILL & 147 & 93 & OKLAHOMA STATE & 79 \\
\hline 37 & NOTRE DAME & 143 & 93 & LOUISIANA STATE & 79 \\
\hline 38 & SOUTHERN CALIFORNIA & 140 & 95 & ALABAMA & 77 \\
\hline 39 & NORTHWESTERN & 139 & 95 & HOUSTON & 77 \\
\hline 40 & TEXAS TECH & 137 & 97 & MASSACHUSETTS & 74 \\
\hline 41 & JOHNS HOPKINS & 135 & 98 & BOSTON COLLEGE & 72 \\
\hline 41 & MANITOBA & 135 & 99 & GUELPH & 69 \\
\hline 43 & DUKE & 131 & 99 & GEORGIA TECH & 69 \\
\hline 43 & TEXAS A\&M & 131 & 101 & OHIO & 68 \\
\hline 45 & NEW MEXICO & 130 & 102 & CONNECTICUT & 67 \\
\hline 46 & MIAMI & 128 & 103 & SUNY-BUFFALO & 66 \\
\hline 47 & TENNESSEE & 125 & 104 & BRIGHAM YOUNG & 59 \\
\hline 48 & ARIZONA & 123 & 104 & ROCHESTER & 59 \\
\hline 49 & CALIFORNIA, DAVIS & 122 & 106 & HOWARD & 57 \\
\hline 50 & MICHIGAN STATE & 121 & 107 & CINCINNATI & 51 \\
\hline 50 & CALIFORNIA, IRVINE & 121 & 107 & COLORADO STATE & 51 \\
\hline 52 & CALIFORNIA, SANTA BARBARA & 118 & 109 & RICE & 50 \\
\hline 53 & IOWA & 117 & 110 & SUNY-ALBANY & 49 \\
\hline 53 & KENTUCKY & 117 & 111 & CASE WESTERN RESERVE & 45 \\
\hline 55 & WASHINGTON U.-ST. LOUIS & 115 & 111 & AUBURN & 45 \\
\hline 55 & COLORADO & 115 & 113 & KENT STATE & 40 \\
\hline 57 & GEORGETOWN & 114 & 114 & SUNY-STONY BROOK & 26 \\
\hline
\end{tabular}


Rank Order Table 19: Total Staff (FTE)

\begin{tabular}{|c|c|c|c|c|c|}
\hline RANK & INSTITUTION & Value & RANK & INSTITUTION & VALUE \\
\hline 1 & HARVARD & 1,214 & 58 & GEORGE WASHINGTON & 245 \\
\hline 2 & YALE & 716 & 59 & VANDERBILT & 244 \\
\hline 3 & TORONTO & 701 & 60 & HAWAII & 238 \\
\hline 4 & COLUMBIA & 657 & 61 & NEW MEXICO & 235 \\
\hline 5 & WISCONSIN & 607 & 62 & OKLAHOMA & 233 \\
\hline 6 & PENNSYLVANIA STATE & 596 & 63 & YORK & 232 \\
\hline 7 & CALIFORNIA, BERKELEY & 590 & 64 & CALIFORNIA, IRVINE & 230 \\
\hline 8 & MICHIGAN & 584 & 65 & FLORIDA STATE & 224 \\
\hline 9 & CALIFORNIA, LOS ANGELES & 580 & 66 & PURDUE & 221 \\
\hline 10 & TEXAS & 560 & 67 & COLORADO & 218 \\
\hline 11 & CORNELL & 524 & 68 & OKLAHOMA STATE & 217 \\
\hline 12 & WASHINGTON & 521 & 68 & OREGON & 217 \\
\hline 13 & ILLINOIS, URBANA & 513 & 68 & SUNY-BUFFALO & 217 \\
\hline 14 & FLORIDA & 487 & 71 & LAVAL & 216 \\
\hline 15 & NEW YORK & 471 & 71 & MANITOBA & 216 \\
\hline 16 & OHIO STATE & 469 & 73 & ROCHESTER & 215 \\
\hline 17 & NORTH CAROLINA & 468 & 74 & TEMPLE & 213 \\
\hline 18 & INDIANA & 415 & 75 & CALIFORNIA, SANTA BARBARA & 210 \\
\hline 19 & RUTGERS & 406 & 76 & CALIFORNIA, DAVIS & 208 \\
\hline 20 & PENNSYLVANIA & 405 & 77 & MIT & 206 \\
\hline 20 & PRINCETON & 405 & 78 & ALABAMA & 203 \\
\hline 22 & BRIGHAM YOUNG & 394 & 79 & BROWN & 200 \\
\hline 23 & MINNESOTA & 391 & 80 & CONNECTICUT & 198 \\
\hline 24 & VIRGINIA & 378 & 81 & NEBRASKA & 197 \\
\hline 25 & SOUTHERN CALIFORNIA & 373 & 82 & DARTMOUTH & 196 \\
\hline 26 & MONTREAL & 365 & 83 & SOUTHERN ILLINOIS & 191 \\
\hline 27 & CALIFORNIA, SAN DIEGO & 361 & 83 & SYRACUSE & 191 \\
\hline 28 & TEXAS A\&M & 355 & 83 & WESTERN ONTARIO & 191 \\
\hline 29 & PITTSBURGH & 353 & 86 & BOSTON COLLEGE & 190 \\
\hline 30 & DUKE & 348 & 86 & MISSOURI & 190 \\
\hline 31 & NORTHWESTERN & 343 & 88 & DELAWARE & 189 \\
\hline 32 & ARIZONA STATE & 340 & 88 & MASSACHUSETTS & 189 \\
\hline 33 & BRITISH COLUMBIA & 339 & 90 & HOUSTON & 188 \\
\hline 34 & TEXAS TECH & 337 & 91 & LOUISIANA STATE & 187 \\
\hline 35 & ALBERTA & 336 & 92 & ILLINOIS, CHICAGO & 185 \\
\hline 36 & UTAH & 323 & 93 & LOUISVILLE & 183 \\
\hline 37 & CHICAGO & 314 & 94 & CINCINNATI & 176 \\
\hline 38 & JOHNS HOPKINS & 299 & 95 & QUEEN'S & 170 \\
\hline 39 & KANSAS & 291 & 95 & WASHINGTON STATE & 170 \\
\hline 40 & ARIZONA & 287 & 97 & MCMASTER & 168 \\
\hline 41 & GEORGIA & 286 & 98 & IOWA STATE & 167 \\
\hline 42 & EMORY & 283 & 99 & WATERLOO & 164 \\
\hline 43 & IOWA & 282 & 100 & OHIO & 161 \\
\hline 44 & BOSTON & 281 & 101 & HOWARD & 157 \\
\hline 45 & MIAMI & 276 & 102 & SASKATCHEWAN & 154 \\
\hline 46 & SOUTH CAROLINA & 274 & 103 & TULANE & 153 \\
\hline 47 & MICHIGAN STATE & 273 & 104 & SUNY-ALBANY & 151 \\
\hline 48 & NORTH CAROLINA STATE & 272 & 105 & CALIFORNIA, RIVERSIDE & 150 \\
\hline 49 & NOTRE DAME & 269 & 106 & VIRGINIA TECH & 145 \\
\hline 50 & KENTUCKY & 268 & 107 & SUNY-STONY BROOK & 144 \\
\hline 50 & MARYLAND & 268 & 108 & GUELPH & 136 \\
\hline 52 & CALGARY & 267 & 109 & CASE WESTERN RESERVE & 134 \\
\hline 53 & GEORGETOWN & 261 & 110 & AUBURN & 123 \\
\hline 54 & WASHINGTON U.-ST. LOUIS & 258 & 110 & COLORADO STATE & 123 \\
\hline 55 & MCGILL & 250 & 110 & GEORGIA TECH & 123 \\
\hline 56 & TENNESSEE & 249 & 113 & RICE & 119 \\
\hline 57 & WAYNE STATE & 246 & 114 & KENT STATE & 110 \\
\hline
\end{tabular}


Rank Order Table 20: Expenditures for Electronic Resources

\begin{tabular}{|c|c|c|c|c|c|}
\hline RANK & INSTITUTION & Value & RANK & INSTITUTION & VALUE \\
\hline 1 & ALBERTA & $14,314,075$ & 58 & TEXAS TECH & $5,854,021$ \\
\hline 2 & COLUMBIA & $11,926,816$ & 59 & FLORIDA STATE & $5,808,581$ \\
\hline 3 & TORONTO & $11,445,449$ & 60 & CALIFORNIA, SAN DIEGO & $5,772,873$ \\
\hline 4 & PENNSYLVANIA STATE & $11,159,991$ & 61 & MIT & $5,764,318$ \\
\hline 5 & JOHNS HOPKINS & $11,143,779$ & 62 & ALABAMA & $5,735,897$ \\
\hline 6 & TEXAS A\&M & $10,543,815$ & 63 & NOTRE DAME & $5,714,316$ \\
\hline 7 & MICHIGAN & $10,437,821$ & 64 & TULANE & $5,648,319$ \\
\hline 8 & HARVARD & $10,204,304$ & 65 & HAWAII & $5,636,156$ \\
\hline 9 & NEW YORK & $9,874,054$ & 66 & OHIO STATE & $5,598,596$ \\
\hline 10 & YALE & $9,418,024$ & 67 & CINCINNATI & $5,554,516$ \\
\hline 11 & MIAMI & $9,391,680$ & 68 & CALIFORNIA, IRVINE & $5,389,920$ \\
\hline 12 & PITTSBURGH & $9,336,254$ & 69 & VIRGINIA & $5,350,099$ \\
\hline 13 & MINNESOTA & $9,327,938$ & 70 & BOSTON COLLEGE & $5,340,070$ \\
\hline 14 & BRITISH COLUMBIA & $9,221,421$ & 71 & BRIGHAM YOUNG & $5,223,576$ \\
\hline 15 & PRINCETON & $8,789,232$ & 72 & GEORGE WASHINGTON & $5,216,058$ \\
\hline 16 & ARIZONA & $8,696,274$ & 73 & CALIFORNIA, LOS ANGELES & $5,150,666$ \\
\hline 17 & WASHINGTON & $8,672,713$ & 74 & OKLAHOMA & $5,066,452$ \\
\hline 18 & NORTH CAROLINA & $8,565,526$ & 75 & GEORGETOWN & $5,003,140$ \\
\hline 19 & DUKE & $8,384,850$ & 76 & BROWN & $4,963,922$ \\
\hline 20 & ARIZONA STATE & $8,355,665$ & 77 & QUEEN'S & $4,960,207$ \\
\hline 21 & TEXAS & $8,338,663$ & 78 & BOSTON & $4,958,895$ \\
\hline 22 & WASHINGTON U.-ST. LOUIS & $7,956,611$ & 79 & VIRGINIA TECH & $4,924,039$ \\
\hline 23 & VANDERBILT & $7,932,073$ & 80 & CALIFORNIA, DAVIS & $4,888,620$ \\
\hline 24 & ILLINOIS, URBANA & $7,857,131$ & 81 & SOUTH CAROLINA & $4,862,112$ \\
\hline 25 & NORTHWESTERN & $7,838,852$ & 82 & AUBURN & $4,851,587$ \\
\hline 26 & TEMPLE & $7,800,437$ & 83 & COLORADO STATE & $4,741,974$ \\
\hline 27 & TENNESSEE & $7,676,038$ & 84 & GEORGIA TECH & $4,634,656$ \\
\hline 28 & MCGILL & $7,667,117$ & 85 & LAVAL & $4,537,986$ \\
\hline 29 & IOWA & $7,665,973$ & 86 & SYRACUSE & $4,509,636$ \\
\hline 30 & CORNELL & $7,605,859$ & 87 & UTAH & $4,500,465$ \\
\hline 31 & INDIANA & $7,561,199$ & 88 & FLORIDA & $4,498,063$ \\
\hline 32 & PENNSYLVANIA & $7,498,556$ & 89 & SUNY-STONY BROOK & $4,475,822$ \\
\hline 33 & PURDUE & $7,345,579$ & 90 & RICE & $4,434,576$ \\
\hline 34 & MONTREAL & $7,232,096$ & 91 & MASSACHUSETTS & $4,372,637$ \\
\hline 35 & CONNECTICUT & $7,174,384$ & 92 & NEBRASKA & $4,351,815$ \\
\hline 36 & MICHIGAN STATE & $7,156,735$ & 93 & SASKATCHEWAN & $4,321,026$ \\
\hline 37 & RUTGERS & $7,060,580$ & 94 & NORTH CAROLINA STATE & $4,299,856$ \\
\hline 38 & CHICAGO & $7,030,483$ & 95 & SOUTHERN ILLINOIS & $4,261,789$ \\
\hline 39 & COLORADO & $6,822,141$ & 96 & WASHINGTON STATE & $4,237,406$ \\
\hline 40 & CALGARY & $6,723,204$ & 97 & MANITOBA & $4,212,515$ \\
\hline 41 & SOUTHERN CALIFORNIA & $6,676,513$ & 98 & NEW MEXICO & $4,203,707$ \\
\hline 42 & WAYNE STATE & $6,674,911$ & 99 & MCMASTER & $4,102,720$ \\
\hline 43 & WISCONSIN & $6,513,942$ & 100 & LOUISVILLE & $4,100,831$ \\
\hline 44 & IOWA STATE & $6,496,636$ & 101 & LOUISIANA STATE & $4,099,957$ \\
\hline 45 & MISSOURI & $6,457,768$ & 102 & GUELPH & $3,846,301$ \\
\hline 46 & CALIFORNIA, BERKELEY & $6,339,903$ & 103 & WATERLOO & $3,836,700$ \\
\hline 47 & DARTMOUTH & $6,289,015$ & 104 & CASE WESTERN RESERVE & $3,818,943$ \\
\hline 48 & KENTUCKY & $6,285,343$ & 105 & ROCHESTER & $3,741,503$ \\
\hline 49 & WESTERN ONTARIO & $6,279,191$ & 106 & CALIFORNIA, SANTA BARBARA & $3,309,681$ \\
\hline 50 & MARYLAND & $6,275,120$ & 107 & OREGON & $3,101,966$ \\
\hline 51 & YORK & $6,155,786$ & 108 & OHIO & $3,025,617$ \\
\hline 52 & EMORY & $6,117,121$ & 109 & HOWARD & $2,547,396$ \\
\hline 53 & SUNY-BUFFALO & $6,051,641$ & 110 & CALIFORNIA, RIVERSIDE & $2,224,276$ \\
\hline 54 & KANSAS & $5,973,543$ & 111 & KENT STATE & $2,143,464$ \\
\hline 55 & DELAWARE & $5,962,493$ & 112 & SUNY-ALBANY & $1,918,903$ \\
\hline 56 & HOUSTON & $5,870,147$ & 113 & ILLINOIS, CHICAGO & $1,530,825$ \\
\hline 57 & GEORGIA & $5,854,147$ & & OKLAHOMA STATE & $\mathrm{UA} / \mathrm{NA}$ \\
\hline
\end{tabular}


Rank Order Table 21: Electronic Resources as a Percentage of Total Library Materials

\begin{tabular}{|c|c|c|c|c|c|}
\hline RANK & INSTITUTION & Value & RANK & INSTITUTION & VALUe \\
\hline 1 & ALBERTA & 85.40 & 58 & CASE WESTERN RESERVE & 56.94 \\
\hline 2 & AUBURN & 84.23 & 59 & CALIFORNIA, IRVINE & 56.80 \\
\hline 3 & GEORGIA TECH & 76.77 & 60 & GEORGIA & 56.48 \\
\hline 4 & WAYNE STATE & 76.71 & 61 & NORTHWESTERN & 56.39 \\
\hline 5 & JOHNS HOPKINS & 76.47 & 62 & BROWN & 56.29 \\
\hline 6 & MISSOURI & 76.34 & 63 & CALIFORNIA, DAVIS & 56.08 \\
\hline 7 & ARIZONA STATE & 74.57 & 64 & WISCONSIN & 55.62 \\
\hline 8 & COLORADO STATE & 72.85 & 65 & $\mathrm{OHIO}$ & 55.37 \\
\hline 9 & FLORIDA STATE & 72.04 & 66 & NEW YORK & 55.11 \\
\hline 10 & VANDERBILT & 71.69 & 67 & MINNESOTA & 54.87 \\
\hline 11 & CONNECTICUT & 70.62 & 68 & BRIGHAM YOUNG & 54.74 \\
\hline 12 & ALABAMA & 69.89 & 69 & CINCINNATI & 54.57 \\
\hline 13 & HAWAII & 69.53 & 70 & MANITOBA & 54.31 \\
\hline 14 & WASHINGTON STATE & 68.87 & 71 & ILLINOIS, URBANA & 54.08 \\
\hline 15 & VIRGINIA TECH & 68.82 & 72 & IOWA & 53.69 \\
\hline 16 & MIAMI & 68.58 & 73 & NORTH CAROLINA & 52.98 \\
\hline 17 & MASSACHUSETTS & 68.14 & 74 & BOSTON & 52.89 \\
\hline 18 & SUNY-BUFFALO & 67.60 & 75 & NEW MEXICO & 52.62 \\
\hline 19 & ARIZONA & 67.46 & 76 & WASHINGTON & 52.07 \\
\hline 20 & NEBRASKA & 67.38 & 77 & IOWA STATE & 51.72 \\
\hline 21 & DARTMOUTH & 66.91 & 78 & MCMASTER & 51.40 \\
\hline 22 & DELAWARE & 66.48 & 79 & INDIANA & 51.11 \\
\hline 23 & GUELPH & 66.10 & 80 & KENT STATE & 50.99 \\
\hline 24 & MARYLAND & 66.06 & 81 & DUKE & 50.97 \\
\hline 25 & PITTSBURGH & 65.72 & 82 & TEXAS TECH & 50.84 \\
\hline 26 & PURDUE & 65.44 & 83 & PENNSYLVANIA & 49.25 \\
\hline 27 & TEMPLE & 65.32 & 84 & TORONTO & 49.10 \\
\hline 28 & LOUISIANA STATE & 64.84 & 85 & NOTRE DAME & 48.69 \\
\hline 29 & WATERLOO & 64.75 & 86 & COLUMBIA & 48.57 \\
\hline 30 & TULANE & 64.56 & 87 & OREGON & 48.57 \\
\hline 31 & SUNY-STONY BROOK & 64.39 & 88 & TEXAS & 47.85 \\
\hline 32 & MIT & 63.68 & 89 & LAVAL & 47.11 \\
\hline 33 & TENNESSEE & 63.19 & 90 & CORNELL & 47.06 \\
\hline 34 & HOWARD & 62.54 & 91 & GEORGE WASHINGTON & 46.67 \\
\hline 35 & WASHINGTON U.-ST. LOUIS & 62.30 & 92 & CALIFORNIA, RIVERSIDE & 46.31 \\
\hline 36 & YORK & 62.11 & 93 & MICHIGAN & 46.26 \\
\hline 37 & KANSAS & 62.08 & 94 & ROCHESTER & 45.26 \\
\hline 38 & WESTERN ONTARIO & 61.65 & 95 & SASKATCHEWAN & 45.00 \\
\hline 39 & UTAH & 61.52 & 96 & NORTH CAROLINA STATE & 44.44 \\
\hline 40 & RUTGERS & 61.51 & 97 & OHIO STATE & 44.20 \\
\hline 41 & MICHIGAN STATE & 60.79 & 98 & LOUISVILLE & 43.22 \\
\hline 42 & CALIFORNIA, SANTA BARBARA & 60.73 & 99 & RICE & 42.39 \\
\hline 43 & PENNSYLVANIA STATE & 60.34 & 100 & GEORGETOWN & 41.66 \\
\hline 44 & BRITISH COLUMBIA & 60.01 & 101 & VIRGINIA & 40.82 \\
\hline 45 & COLORADO & 59.87 & 102 & PRINCETON & 40.07 \\
\hline 46 & SOUTH CAROLINA & 59.70 & 103 & CHICAGO & 38.90 \\
\hline 47 & SYRACUSE & 59.56 & 104 & SOUTHERN CALIFORNIA & 38.47 \\
\hline 48 & QUEEN'S & 59.56 & 105 & SUNY-ALBANY & 36.91 \\
\hline 49 & TEXAS A\&M & 59.34 & 106 & FLORIDA & 36.68 \\
\hline 50 & HOUSTON & 59.27 & 107 & EMORY & 36.03 \\
\hline 51 & MONTREAL & 59.05 & 108 & CALIFORNIA, LOS ANGELES & 35.62 \\
\hline 52 & MCGILL & 59.03 & 109 & CALIFORNIA, BERKELEY & 35.52 \\
\hline 53 & SOUTHERN ILLINOIS & 58.61 & 110 & OKLAHOMA & 35.17 \\
\hline 54 & CALIFORNIA, SAN DIEGO & 58.45 & 111 & HARVARD & 28.91 \\
\hline 55 & KENTUCKY & 58.36 & 112 & YALE & 24.08 \\
\hline 56 & BOSTON COLLEGE & 58.29 & 113 & ILLINOIS, CHICAGO & 14.33 \\
\hline 57 & CALGARY & 57.03 & & OKLAHOMA STATE & $\mathrm{UA} / \mathrm{NA}$ \\
\hline
\end{tabular}


Rank Order Table 22: Library InVestment Index

\begin{tabular}{|c|c|c|c|c|c|}
\hline RANK & INSTITUTION & VAlue & RANK & INSTITUTION & VAlue \\
\hline 1 & HARVARD & 6.1121 & 58 & TEMPLE & -0.2690 \\
\hline 2 & YALE & 4.0070 & 59 & IOWA STATE & -0.2692 \\
\hline 3 & COLUMBIA & 2.3674 & 60 & KANSAS & -0.2878 \\
\hline 4 & TORONTO & 2.0961 & 61 & UTAH & -0.3031 \\
\hline 5 & MICHIGAN & 1.6485 & 62 & GEORGIA & -0.3159 \\
\hline 6 & CALIFORNIA, BERKELEY & 1.5971 & 63 & COLORADO & -0.3498 \\
\hline 7 & PENNSYLVANIA STATE & 1.3643 & 64 & KENTUCKY & -0.3523 \\
\hline 8 & CALIFORNIA, LOS ANGELES & 1.3616 & 65 & CALIFORNIA, IRVINE & -0.3734 \\
\hline 9 & PRINCETON & 1.3099 & 66 & BOSTON & -0.3826 \\
\hline 10 & TEXAS & 1.1416 & 67 & SUNY-BUFFALO & -0.3881 \\
\hline 11 & NEW YORK & 1.1373 & 68 & WAYNE STATE & -0.4188 \\
\hline 12 & CORNELL & 1.1319 & 69 & BROWN & -0.4274 \\
\hline 13 & WASHINGTON & 1.0579 & 70 & ILLINOIS, CHICAGO & -0.4354 \\
\hline 14 & WISCONSIN & 0.9999 & 71 & CINCINNATI & -0.4386 \\
\hline 15 & ILLINOIS, URBANA & 0.8865 & 72 & NEW MEXICO & -0.4599 \\
\hline 16 & ALBERTA & 0.8823 & 73 & LAVAL & -0.4827 \\
\hline 17 & NORTH CAROLINA & 0.8619 & 74 & HOUSTON & -0.4846 \\
\hline 18 & MINNESOTA & 0.8520 & 75 & BOSTON COLLEGE & -0.4868 \\
\hline 19 & SOUTHERN CALIFORNIA & 0.7630 & 76 & MANITOBA & -0.4905 \\
\hline 20 & PENNSYLVANIA & 0.7367 & 77 & CALIFORNIA, DAVIS & -0.4932 \\
\hline 21 & DUKE & 0.7246 & 78 & DARTMOUTH & -0.4989 \\
\hline 22 & OHIO STATE & 0.6808 & 79 & LOUISVILLE & -0.5242 \\
\hline 23 & TEXAS A\&M & 0.6113 & 80 & HAWAII & -0.5253 \\
\hline 24 & CHICAGO & 0.5229 & 81 & ROCHESTER & -0.5395 \\
\hline 25 & EMORY & 0.4919 & 82 & WESTERN ONTARIO & -0.5486 \\
\hline 26 & BRITISH COLUMBIA & 0.4854 & 83 & SOUTH CAROLINA & -0.5507 \\
\hline 27 & VIRGINIA & 0.3911 & 84 & DELAWARE & -0.5573 \\
\hline 28 & INDIANA & 0.3862 & 85 & SASKATCHEWAN & -0.5824 \\
\hline 29 & RUTGERS & 0.3114 & 86 & CALIFORNIA, SANTA BARBARA & -0.5969 \\
\hline 30 & NORTHWESTERN & 0.2010 & 87 & SYRACUSE & -0.6023 \\
\hline 31 & PITTSBURGH & 0.1885 & 88 & OKLAHOMA STATE & -0.6288 \\
\hline 32 & ARIZONA & 0.1731 & 89 & ALABAMA & -0.6433 \\
\hline 33 & IOWA & 0.1552 & 90 & MISSOURI & -0.6563 \\
\hline 34 & MONTREAL & 0.1357 & 91 & MCMASTER & -0.6572 \\
\hline 35 & CALIFORNIA, SAN DIEGO & 0.1255 & 92 & RICE & -0.6763 \\
\hline 36 & JOHNS HOPKINS & 0.1254 & 93 & QUEEN'S & -0.6830 \\
\hline 37 & WASHINGTON U.-ST. LOUIS & 0.0486 & 94 & FLORIDA STATE & -0.7434 \\
\hline 38 & GEORGETOWN & 0.0388 & 95 & OREGON & -0.7482 \\
\hline 39 & FLORIDA & 0.0364 & 96 & TULANE & -0.7862 \\
\hline 40 & MCGILL & 0.0355 & 97 & MASSACHUSETTS & -0.7896 \\
\hline 41 & NORTH CAROLINA STATE & 0.0345 & 98 & COLORADO STATE & -0.7983 \\
\hline 42 & CONNECTICUT & -0.0276 & 99 & SOUTHERN ILLINOIS & -0.7985 \\
\hline 43 & MIAMI & -0.0373 & 100 & NEBRASKA & -0.8019 \\
\hline 44 & GEORGE WASHINGTON & -0.0776 & 101 & SUNY-STONY BROOK & -0.8532 \\
\hline 45 & MICHIGAN STATE & -0.0791 & 102 & CASE WESTERN RESERVE & -0.8595 \\
\hline 46 & TEXAS TECH & -0.1027 & 103 & WASHINGTON STATE & -0.8613 \\
\hline 47 & BRIGHAM YOUNG & -0.1097 & 104 & VIRGINIA TECH & -0.8636 \\
\hline 48 & MARYLAND & -0.1103 & 105 & LOUISIANA STATE & -0.9011 \\
\hline 49 & CALGARY & -0.1285 & 106 & WATERLOO & -0.9277 \\
\hline 50 & MIT & -0.1399 & 107 & CALIFORNIA, RIVERSIDE & -0.9285 \\
\hline 51 & NOTRE DAME & -0.1541 & 108 & $\mathrm{OHIO}$ & -0.9384 \\
\hline 52 & PURDUE & -0.1679 & 109 & SUNY-ALBANY & -0.9634 \\
\hline 53 & VANDERBILT & -0.1813 & 110 & GEORGIA TECH & -0.9658 \\
\hline 54 & ARIZONA STATE & -0.1871 & 111 & GUELPH & -0.9703 \\
\hline 55 & OKLAHOMA & -0.2015 & 112 & AUBURN & -0.9809 \\
\hline 56 & TENNESSEE & -0.2264 & 113 & KENT STATE & -0.9850 \\
\hline 57 & YORK & -0.2654 & 114 & HOWARD & -1.1781 \\
\hline
\end{tabular}

Rank Order Tables of University Libraries · 95 



\section{ARL Statistics Questionnaire 2008-2009}

\section{InSTRUCTIONS FOR COMPLeTING THE QUeSTIONNAIRE}

\section{General Instructions}

Definitions of statistical categories can be found in NISO Z39.7-2004, Information Services and Use: Metrics \& statistics for libraries and information providers--Data Dictionary (http://www.niso.org/). ARL has been modifying the interpretation of the standard definitions to address questions posed by library staff at various member institutions that complete the survey and with feedback from the ARL Statistics and Assessment Committee (http://www.arl.org/stats/aboutstats/index.shtml).

Please do not use decimals. All figures should be rounded to the nearest whole number.

Please respond to every question. If an exact figure cannot be provided, use NA/UA to indicate that the figure is either unavailable or not applicable. If the appropriate answer is zero or none, use $\mathbf{0}$.

Although the form allows for data to be entered from both main and branch campuses, an effort should be made to report figures for the main campus only. (The U.S. National Center for Education Statistics, Integrated Postsecondary Education Data System (IPEDS) defines a branch institution as "a campus or site of an educational institution that is not temporary, is located in a community beyond a reasonable commuting distance from its parent institution, and offers organized programs of study, not just courses"). If figures for libraries located at branch campuses are reported, please specify which branch libraries are included and which ones are excluded in the notes below.

A branch library is defined as an auxiliary library service outlet with quarters separate from the central library of an institution, which has a basic collection of books and other materials, a regular staffing level, and an established schedule. A branch library is administered either by the central library or (as in the case of some law and medical libraries) through the administrative structure of other units within the university. Departmental study/reading rooms are not included.

The questionnaire assumes a fiscal year ending June 30, 2009. If your fiscal year is different, please indicate this in the notes below by adjusting the reporting period.

Footnotes. Explanatory footnotes will be included with the published statistics. Provide any notes you may have in the footnotes area at the end of the survey. Reporting libraries are urged to record there any information that would clarify the figures submitted in that line, e.g., the inclusion and exclusion of branch campus libraries. Please make an effort to word your footnotes in a manner consistent with notes appearing in the published report, so that the ARL Office can interpret your footnotes correctly. Please use a concise sentence/paragraph format when writing footnotes - do not use "bullets" or make a "bullet list."

\section{$\underline{\text { Specific Instructions }}$}

Questions 1-1b. Volumes:

Question 1. Volumes in Library. Use the ANSI/NISO Z39.7-2004 definition for volume as follows:

a single physical unit of any printed, typewritten, handwritten, mimeographed, or processed work, distinguished from other units by a separate binding, encasement, portfolio, or other clear distinction, which has been cataloged, classified, and made ready for use, and which is 
Include duplicates and bound volumes of periodicals. For purposes of this questionnaire, unclassified bound serials arranged in alphabetical order are considered classified. Exclude microforms, maps, nonprint materials, and uncataloged items. If any of these items cannot be excluded, please provide an explanatory footnote

Include government document volumes that are accessible through the library's catalogs regardless of whether they are separately shelved. "Classified" includes documents arranged by Superintendent of Documents, CODOC, or similar numbers. "Cataloged" includes documents for which records are provided by the library or downloaded from other sources into the library's card or online catalogs. Documents should, to the extent possible, be counted as they would if they were in bound volumes (e.g., 12 issues of an annual serial would be one or two volumes). Title and piece counts should not be considered the same as volume counts. If a volume count has not been kept, it may be estimated through sampling a representative group of title records and determining the corresponding number of volumes, then extrapolating to the rest of the collection. As an alternative, an estimate may be made using the following formulae:

\section{2 documents pieces per foot \\ 10 "traditional" volumes per foot \\ 5.2 documents pieces per volume}

Include e-book units, as long as these e-books are owned or leased and have been cataloged by your library. Include electronic books purchased through vendors such as NetLibrary ${ }^{\circledR}$ or Books $24 \times 7$, and e-books that come as part of aggregate services. Include individual titles of e-book sets that are treated as individual reference sources. Include locally digitized electronic books and electronic theses and dissertations. Provide a footnote explaining how many e-books you are reporting, preferably by specifying the products and the number of titles in a note.

Include volumes purchased collectively where the cost is shared at the time of purchase.

If either formulas or sampling are used for deriving your count, please indicate in a footnote.

Question 1b. Volumes Added. Include only volumes cataloged, classified, and made ready for use. Include government documents if they have been included in the count of volumes on line 1a. Do not include as part of Volumes Added Gross any government documents or other collections (such as large gift collections or e-book packages, EBBO, etc.) that were added to the collection as the result of a one time download or addition to the OPAC. Include these items in Volumes Held of the previous year (Line 1a) and provide a footnote explaining the revision of Line 1a.

Question 2. Titles Held. Use the ANSI/NISO Z39.7-2004 definition for title as follows:

The designation of a separate bibliographic whole, whether issued in one or several volumes .... Titles are defined according to the Anglo-American Cataloging Rules. A book or serial title may be distinguished from other such titles by its unique International Standard Book Number (ISBN) or International Standard Serial Number (ISSN). This definition applies equally to print, audiovisual, and other library materials. For unpublished works, the term is used to designate a manuscript collection or an archival record series. Two subscriptions to Science magazine, for example, are counted as one title. When vertical file materials are counted, a file folder is considered a title. 
Report the total number of unique titles cataloged, classified and made ready for use. The number of titles reported here is for the number of volumes reported under line (1). Include e-books as specified above in question (1). For those reporting a bibliographic volume under line (1), their title count may be exactly the same as their volume count.

Question 3. Monographic Volumes Purchased. Report number of volumes purchased; do not include volumes received or cataloged. Include all volumes for which an expenditure was made during 2008-09, including volumes paid for in advance but not received during the fiscal year. Include monographs in series and continuations. Include e-books that fit the NetLibrary ${ }^{\circledR}$ model, i.e., electronic manifestations of physical entities and/or units; provide a footnote explaining how many e-books you are reporting, preferably by specifying the products and the number of titles. If only number of titles purchased can be reported, please report the data and provide an explanatory footnote.

Question 4: Basis of Volume Count. A physical count is a piece count; a bibliographic count is a catalog record count.

Questions 5. Serials. Use the following definition adapted from AACR2 for a serial:
A bibliographic resource issued in a succession of discrete parts, usually bearing numbering, that has no predetermined conclusion. Examples of serials include
journals, magazines, electronic journals, continuing directories, annual reports, newspapers, and monographic series.

Report the total number of unique serial titles, NOT SUBSCRIPTIONS, that you currently acquire and to which you provide access. Do not include duplicate counts of serial titles. Report each title once, regardless of how many subscriptions or means of access you provide for that title. Exclude unnumbered monographic and publishers' series. Electronic serials acquired as part of a bundle or an aggregated package should be counted at the title level, even if they are not cataloged, as long as the title is made accessible directly by the library (e.g., through a finding aid). If access is provided only through the overall platform or aggregator, do not report the individual titles but count the package as a single title.

Question 5a. Serial titles currently purchased. In the case of consortial agreements, count under 'serial titles currently purchased' those titles for which the library pays any amount from its budgeted expenditures. Include all titles that are part of bundles or aggregated packages, even if your library makes a partial payment for access to those titles. If a purchased title includes electronic access to the title, count that title ONLY ONCE (DEDUPED) as electronic only. If a database includes full-text and abstracted titles, the number of full-text titles can be counted.

Question 5b. Serial titles: Not Purchased. Report other titles that your library receives and does not pay for directly under 'serial titles received but not purchased.' These titles may include exchanges, gifts, etc.

If serial titles have been purchased through a consortium whose budget is centrally funded and independent from the library's budget, these serials should be reported under 'serial titles currently received but not purchased.' If within a purchased or aggregated package it cannot be determined that some titles are not purchased, report all titles as purchased.

Freely accessible titles are those your library provides direct access to via cataloging records or through online serial lists of other finding aids.

To the extent possible, report all government document serials separately in (5b.iv).

If separate counts of non-purchased and purchased serial titles are not available, report only the total number of serial titles currently purchased and received on line (5), and report NA/UA for lines (5a) and (5b). 
Question 7. Microforms. Report the total number of physical units: reels of microfilm, microcards, and microprint and microfiche sheets. Include all government documents in microform; provide a footnote if documents are excluded.

Question 8. Government documents. Report the total number of physical units (pieces) of government documents in paper format that have not been counted elsewhere. Include local, state, national, and international documents; include documents purchased from a commercial source if shelved with separate documents collections and not counted above. Include serials and monographs. To estimate pieces from a measurement of linear feet, use the formula 1 foot $=52$ pieces and indicate in a footnote that the count is based on this estimate. Exclude microforms and non-print formats such as maps or CD-ROMs. Adjust line (1a), i.e., last year's Volumes Held, and provide a footnote if you are adding records to the OPAC for government documents previously held but not counted as part of Volumes Held line (1a).

Question 9. Computer files. Include the number of pieces of computer-readable disks, tapes, CD-ROMs, and similar machinereadable files comprising data or programs that are locally held as part of the library's collections available to library clients. Examples are U.S. Census data tapes, sample research software, locally-mounted databases, and reference tools on CD-ROM, tape or disk. Exclude bibliographic records used to manage the collection (i.e., the library's own catalog in machine-readable form), library system software, and microcomputer software used only by the library staff.

Question 10. Manuscripts and archives. Include both manuscripts and archives measured in linear feet.

Question 11. Cartographic materials. Include the numbers of pieces of two- and three-dimensional maps and globes. Include satellite and aerial photographs and images.

Question 12. Graphic materials. Include the number of pieces of prints, pictures, photographs, postcards, slides, transparencies, film strips, and the like.

Question 13. Audio materials. Include the number of pieces of audiocassettes, phonographic discs, audio compact discs, reel-toreel tapes, and other sound recordings.

Question 14. Film and video materials. Include the number of pieces of motion pictures, videocassettes, video laser discs, and similar visual materials.

Questions 15-21. Expenditures. Report all expenditures of funds that come to the library from the regular institutional budget, and from sources such as research grants, special projects, gifts and endowments, and fees for service. (For question (18), include nonlibrary funds; see instruction for question (18). Do not report encumbrances of funds that have not yet been expended. Canadian libraries should report expenditures in Canadian dollars. (For your information, if interested in determining figures in U.S. dollars, divide Canadian dollar amounts by 1.1667, the average monthly noon exchange rate published in the Bank of Canada Review for the period July 2008-June 2009). Please round figures to the nearest dollar.

Question 16a. Monographs. Report expenditures for volumes purchased counted on line (3).

Question 16b. Serial titles. Report expenditures for serial titles counted on line (5a). Exclude unnumbered monographic and publishers' series, and encumbrances.

Question 16c. Other library materials. Include expenditures for all materials not reported in Questions (16a) and (16b), e.g., backfiles of serials, charts and maps, audiovisual materials, manuscripts, etc. If expenditures for these materials are included in lines (16a) and/or (16b) and cannot be disaggregated, please report U/A and provide a footnote. Do not include encumbrances.

Question 16d. Miscellaneous expenditures. Include any other materials funds expenditures not included in questions $100 \cdot$ ARL Statistics 2008-2009 
(16a)-(16c), e.g., expenditures for bibliographic utilities, literature searching, security devices, memberships for the purposes of publications, etc. Please list categories, with amounts, in a footnote. Note: If your library does not use materials funds for nonmaterials expenditures - i.e., if those expenditures are included in "Other Operating Expenditures"- report 0, not NA/UA, on line (16d).

Question 17. Contract Binding. Include only contract expenditures for binding done outside the library. If all binding is done inhouse, state this fact and give in-house expenditures in a footnote; do not include personnel expenditures. Note: this figure should also be reported in the 2008-09 ARL Preservation Survey, question (8b).

Questions 18. Salaries and wages. Exclude fringe benefits. If professional, support staff and student salaries cannot be separated, enter NA/UA, in lines (18a), (18b) and (18c) and enter total staff salaries in line (18).

Question 18c. Salaries and wages: Student Assistants. Report 100\% of student wages regardless of budgetary source of funds. Include federal and local funds for work study students.

Question 20. Other operating expenditures. Exclude expenditures for buildings, maintenance, and fringe benefits.

Questions 22-26. Electronic expenditures. These items are intended to indicate what portion of your institution's total library expenditures are dedicated to electronic resources and services. Please use the Footnotes to indicate any electronic materials expenditures you believe not to be covered by these questions. Many expenditures recorded in these questions should have been included in question (21), total library expenditures.

Question 22. One-time electronic resource purchases. Report expenditures that are not current serials (i.e. are non-subscription, one-time, or monographic in nature) for software and machine-readable materials considered part of the collections. Examples include periodical backfiles, literature collections, one-time costs for JSTOR membership, etc. Expenditures reported here may be derived from any of the following categories: Monographs (16a), Other Library Materials (16c), Miscellaneous (16d), or Other Operating Expenditures (20).

Question 23. Ongoing electronic resource purchases. Report subscription expenditures (or those which are expected to be ongoing commitments) for serial publications whose primary format is electronic and for online searches of remote databases such as OCLC FirstSearch, DIALOG, Lexis-Nexis, etc. Examples include paid subscriptions for electronic journals and indexes/abstracts available via the Internet, CD-ROM serials, and annual access fees for resources purchased on a "one-time" basis, such as literature collections, JSTOR membership, etc. Not all items whose expenditures are counted here will be included in Serial titles currently received question (5) or Serials Expenditures question (16b).

Question 24. Bibliographic Utilities, Networks, and Consortia. Because it is increasingly common for ARL Libraries to enter into consortial arrangements to purchase access to electronic resources, both "Library" and "External" expenditure blanks and instructions are provided. Please use afootnote to describe expenditures that you believe are not covered by the question, or situations that do not seem to fit the instructions.

Question 24a. From internal library sources. Report expenditures paid by the Library for services provided by national, regional, and local bibliographic utilities, networks, and consortia, such as OCLC and RLG, unless for user database access and subscriptions, which should be reported in questions (22) or (23). Include only expenditures that are part of Other Operating Expenditures (Q20).

Question 24b. From external sources. If your library receives access to computer files, electronic serials or search services through one or more centrally-funded system or consortial arrangements for which it does not pay fully and/or directly (for example, funding is provided by the state on behalf of all members), enter the amount paid by external bodies on its behalf. If the specific dollar amount is not known, but the total student FTE for the consortium and amount spent for the academic members are known, divide the overall amount spent by your institution's share of the total student FTE. 
Question 25. Computer hardware and software. Report expenditures from the library budget for computer hardware and software used to support library operations, whether purchased or leased, mainframe or microcomputer, and whether for staff or public use. Include expenditures for: maintenance; equipment used to run information service products when those expenditures can be separated from the price of the product; telecommunications infrastructure costs, such as wiring, hubs, routers, etc. Include only expenditures that are part of Other Operating Expenditures (20).

Question 26. Document Delivery/Interlibrary Loan. Report expenditures for document delivery and interlibrary loan services (both borrowing and lending). Include fees paid for photocopies, costs of telefacsimile transmission, royalties and access fees paid to provide document delivery or interlibrary loan. Include fees paid to bibliographic utilities if the portion paid for interlibrary loan can be separately counted. Include only expenditures that are part of Miscellaneous Materials Expenditures (16d) or Other Operating Expenditures (20), and only for those ILL/DD programs with data recorded in Questions (35)-(36).

Questions 27. Personnel. Report the number of FTE (full-time equivalent) staff in filled positions, or positions that are only temporarily vacant. ARL defines temporarily vacant positions as positions that were vacated during the fiscal year for which ARL data were submitted, for which there is a firm intent to refill, and for which there are expenditures for salaries reported on line (18).

Include cost recovery positions and staff hired for special projects and grants, but provide an explanatory footnote indicating the number of such staff. If such staff cannot be included, provide a footnote. To compute full-time equivalents of part-time employees and student assistants, take the total number of hours per week (or year) worked by part-time employees in each category and divide it by the number of hours considered by the reporting library to be a full-time work week (or year). Round figures to the nearest whole numbers.

Question 27a. Professional Staff. Since the criteria for determining professional status vary among libraries, there is no attempt to define the term "professional." Each library should report those staff members it considers professional, including, when appropriate, staff who are not librarians in the strict sense of the term, for example computer experts, systems analysts, or budget officers.

Question 27b. Support Staff. Report the total FTE (see instruction (27) of staff not included in (27a).

Question 27c. Student Assistants. Report the total FTE (see instruction Q27) of student assistants employed on an hourly basis whose wages are paid from funds under library control or from a budget other than the library's, including federal work-study programs. Exclude maintenance and custodial staff.

Question 28. Number of staffed library service points. Count the number of staffed public service points in the main library and in all branch libraries reported in this inventory, including reference desks, information desks, circulation, current periodicals, reserve rooms, reprographic services (if staffed as a public facility), etc. Report the number of designated locations, not the number of staff.

Question 29. Number of weekly public service hours. Report an unduplicated count of the total public service hours per typical full-service week (i.e., no holidays or other special accommodations) across both main library and branches using the following method (corresponds to IPEDS): If a library is open from 9:00 a.m. to 5:00 p.m. Monday through Friday, it should report 40 hours per week. If several of its branches are also open during these hours, the figure remains 40 hours per week. Should Branch A also be open one evening from 7:00 p.m. to 9:00 p.m., the total hours during which users can find service somewhere within the system becomes 42 hours per week. If Branch B is open the same hours on the same evening, the count is still 42, but if Branch B is open two hours on another evening, or remains open two hours later, the total is then 44 hours per week. Exclude 24-hour unstaffed reserve or similar reading rooms. The maximum total is 168 (i.e., a staffed reading room open 7 days per week, 24 hours per day).

Questions 30-31. Instruction. Sampling based on a typical week may be used to extrapolate TO A FULL YEAR for Questions (30) and (31). Please indicate if responses are based on sampling. 
Question 30. Presentations to Groups. Report the total number of sessions during the year of presentations made as part of formal bibliographic instruction programs and through other planned class presentations, orientation sessions, and tours. If the library sponsors multi-session or credit courses that meet several times over the course of a semester, each session should be counted. Presentations to groups may be for either bibliographic instruction, cultural, recreational, or educational purposes. Presentations both on and off the premises should be included as long as they are sponsored by the library. Do not include meetings sponsored by other groups using library meeting rooms. Do not include training for library staff; the purpose of this question is to capture information about the services the library provides for its clientele. Please indicate if the figure is based on sampling.

Question 31. Participants in Group Presentations. Report the total number of participants in the presentations reported on line (30). For multi-session classes with a constant enrollment, count each person only once. Personal, one-to-one instruction in the use of sources should be counted as reference transactions on line (32). Please indicate if the figure is based on sampling. Use a footnote to describe any special situations.

Question 32. Reference Transactions. Report the total number of reference transactions. A reference transaction is an information contact that involves the knowledge, use, recommendations, interpretation, or instruction in the use of one or more information sources by a member of the library staff. The term includes information and referral service. Information sources include (a) printed and nonprinted material; (b) machine-readable databases (including computer-assisted instruction); (c) the library's own catalogs and other holdings records; (d) other libraries and institutions through communication or referral; and (e) persons both inside and outside the library. When a staff member uses information gained from previous use of information sources to answer a question, the transaction is reported as a reference transaction even if the source is not consulted again.

If a contact includes both reference and directional services, it should be reported as one reference transaction. Include virtual reference transactions (e.g., e-mail, WWW form, chat). Duration should not be an element in determining whether a transaction is a reference transaction. Sampling based on a typical week may be used to extrapolate TO A FULL YEAR for Question 32. Please indicate if the figure is based on sampling.

EXCLUDE SIMPLE DIRECTIONAL QUESTIONS. A directional transaction is an information contact that facilitates the logistical use of the library and that does not involve the knowledge, use, recommendations, interpretation, or instruction in the use of any information sources other than those that describe the library, such as schedules, floor plans, and handbooks.

Questions 33-34. Circulation. For Question (33), count the number of initial circulations during the fiscal year from the general collection for use usually (although not always) outside the library. Do not count renewals. Include circulations to and from remote storage facilities for library users (i.e., do not include transactions reflecting transfers or stages of technical processing). Count the total number of items lent, not the number of borrowers.

For Question (34), report total circulation for the fiscal year including initial transactions reported on line (33) and renewal transactions. Exclude reserve circulations; these are no longer reported.

Questions 35-36. Interlibrary Loans. Report the number of requests for material (both returnables and non-returnables) provided to other libraries on line (35) and the number of filled requests received from other libraries or providers on line (36). On both lines, include originals, photocopies, and materials sent by telefacsimile or other forms of electronic transmission. Include patron-initiated transactions. Exclude requests for materials locally owned and available on the shelves or electronically. Do not include transactions between libraries covered by this questionnaire. 
Question 37. PhD Degrees. Report the number awarded during the 2007-08 fiscal year. Please note that only the number of $\underline{\mathrm{Ph} . \mathrm{D}}$. degrees are to be counted. Statistics on all other advanced degrees (e.g., D.Ed., D.P.A., M.D., J.D.) should not be reported in this survey. If you are unable to provide a figure for Ph.D.s only, please add a footnote.

Question 38. PhD Fields. For the purposes of this report, Ph.D. fields are defined as the specific discipline specialties enumerated in the U.S. Department of Education's Integrated Postsecondary Education Data System (IPEDS) “Completions" Survey. Although the IPEDS form requests figures for all doctoral degrees, only fields in which $\underline{\mathrm{PhDs}}$ are awarded should be reported on the ARL questionnaire. Any exceptions should be footnoted.

Question 39. Instructional Faculty. Instructional faculty are defined by the U.S. Dept. of Education as:

members of the instruction/research staff who are employed full-time as defined by the institution, including faculty with released time for research and faculty on sabbatical leave.

Full-time counts generally exclude faculty who are employed to teach fewer than two semesters, three quarters, two trimesters, or two four-month sessions; replacements for faculty on sabbatical leave or leave without pay; faculty for preclinical and clinical medicine; faculty who are donating their services; faculty who are members of military organizations and paid on a different pay scale from civilian employees; academic officers, whose primary duties are administrative; and graduate students who assist in the instruction of courses. Please be sure the number reported, and the basis for counting, are consistent with those for 2006-07 (unless in previous years faculty were counted who should have been excluded according to the above definition). Please footnote any discrepancies.

Questions 40-43. Enrollment. U.S. libraries should use the Fall 2008 enrollment figures reported to the Department of Education on the Integrated Postsecondary Education Data System survey. Please check these figures against the enrollment figures reported to ARL last year to ensure consistency and accuracy. Note: In the past, the number of part-time students reported was FTE; the number now reported to IPEDS is a head count of part-time students. Canadian libraries should note that the category "graduate students" as reported here includes all post-baccalaureate students.

\section{FOOTNOTES}

Please consult the data entry Web interface (www.arlstatistics.org) for a copy of last year's footnotes. These can be found under "Data Repository" after you login into www.arlstatistics.org. Explanatory footnotes will be included with the published statistics. Reporting libraries are urged to record in the footnote section any information that would clarify the figures submitted, e.g., the inclusion and exclusion of branch campus libraries (see the "General Instructions" for definition of branch campus libraries). Please make an effort to word your footnotes in a manner consistent with notes appearing in the published report, so that the ARL Office can interpret your footnotes correctly.

NOTE: Any change over 10\% in any answer to any of the survey's questions over the preceding year's response (2007-08) should be addressed with a footnote.

Submit the completed questionnaire by

October 15, 2009.

For assistance, please e-mail Martha Kyrillidou (martha@arl.org) or Les Bland (les@arl.org)

Tel. (202) 296-2296.

104 · ARL Statistics 2008-2009 


\section{ARL Statistics WorksheEt 2008-2009}

This worksheet is designed to help you plan your submission for the 2008-2009 ARL Statistics. The figures on this worksheet should be similar to those in the "Summary" page of your web form, except in cases where data are unavailable. If an exact figure is unavailable, use "NA/UA". If the appropriate answer is zero or none, use " 0. "

Reporting Institution Date Returned to ARL

Report Prepared by (name)

Title

Email address Phone number

Contact person (if different)

Title

Email address Phone number

PAGE ONE - VOLUMES AND TITLES:

1. Volumes held June 30, 2009 (1.a + 1.b)

(1)

1a. Volumes held June 30, 2008

(1.a)

1b. Volumes added during the year (1.b.i - 1.b.ii)

(i) Volumes added - Gross

(ii) Volumes withdrawn during year

(1.b.ii)

2. Titles held June 30, 2009

(2)

3. Number of monographic volumes purchased

(3)

4. Basis of volume count is:

(4)

Physical

Bibliographic 
PAGE TWO - OTHER COLLECTIONS

SERIALS

5. Total number of serial titles currently received, including periodicals $(5 . a+5 . b)$

(5)

5a. Number of serial titles currently purchased $(5 a . i+5 a . i i)(5 a)$

5a.i Electronic

5a.ii Print (and other format) serials purchased

(5a.ii)

$5 \mathrm{~b}$. Number of serial titles currently received but not purchased

$$
(5 b . i+5 b . i i+5 b . i i i+5 b . i v)
$$

5b.i Consortial

5b.ii Freely accessible

5b.iii Print (and other format) - Exchanges,

(5b.iii) gifts, etc.

5b.iv Government documents

(5b.iv)

6. Government documents are included in count of Current Serials?

(6) Yes No

\section{OTHER LIBRARYMATERIALS}

7. Microform units

(7)

8. Government documents not counted elsewhere

(8)

9. Computer files

(9)

10. Manuscripts and archives (linear ft.)

(10)

\section{AUDIOVISUAL MATERIALS}

11. Cartographic

12. Graphic

13. Audio

14. Film and Video 
PAGE THREE - EXPENDITURES

15. Are the below figures reported in Canadian dollars?

(15) Yes No

16. Total Library Materials Expenditures $(16 \cdot a+16 . b+16 . c+16 . d)$
16a. Monographs
(16a)
16b. Serial titles, including periodicals
(16b)
16c. Other Library Materials
(16c)
16d. Miscellaneous
(16d)

17. Contract binding

(17)

18. Total Salaries and Wages $(18 . a+18 . b+18 . c)$

(18)

18a. Professional staff

(18a)

18b. Support staff

18c. Student assistants

(18c)

19. Fringe benefits are included in expenditures for salaries and wages? (19) Yes No

20. Other operating expenditures

(20)

21. Total library expenditures $\quad(16+17+18+20)$

(21)

\section{ELECTRONIC MATERIALS EXPENDITURES}

22. One-time electronic resource purchases

23. Ongoing electronic resource purchases (e.g., subscriptions, annual license fees)

(23)

24. Bibliographic Utilities, Networks, and Consortia

24a. From internal library sources

(24a)

24b. From external sources

25. Computer hardware and software

(25)

26. Document Delivery/Interlibrary Loan

(26) 
PAGE FOUR - PERSONNEL AND PUBLIC SERVICES

PERSONNEL (Round figures to nearest whole number.)

27. Total Staff FTE $(27 . a+27 . b+27 . c)$

27a. Professional staff, FTE

(27a)

27b. Support staff, FTE

(27b)

27c. Student assistants, FTE

$(27 \mathrm{c})$

\section{STAFFED SERVICE POINTS AND HOURS}

28. Number of staffed library service points

(28)

29. Number of weekly public service hours

(29)

\section{INSTRUCTION}

30. Number of library presentations to groups

(30)

30a. Is the library presentations figure based on sampling?

(30a) Yes No

31. Number of total participants in group presentations reported in line 30

31a. Is the total participants in group presentations figure based on sampling?

(31a) Yes No

\section{REFERENCE}

32. Number of reference transactions

32a. Is the reference transactions figure based on sampling?

(32a) Yes No 


\section{CIRCULATION}

33. Number of initial circulations (excluding reserves)

34. Total circulations (initial and renewals, excluding reserves)

\section{INTERLIBRARYLOANS}

\section{Total number of filled requests provided to other libraries}

36. Total number of filled requests received from other libraries or providers

\section{PhD DEGREES AND FACULTY}

37. Number of PhDs awarded in FY2008-2009

38. Number of fields in which PhDs can be awarded

39. Number of full-time instructional faculty in FY2008-2009

ENROLLMENT - FALL 2008

(Line numbers refer to IPEDS survey form.)

40. Full-time students, undergraduate and graduate

(Add line 8, columns $15 \mathcal{E} 16$, and line 14, columns $15 \mathcal{E} 16$. .)

41. Part-time students, undergraduate and graduate

(Add line 22, columns $15 \& 16$, and line 28, columns $15 \& 16$.)

43. Part-time graduate students (Line 28, columns $15 \mathcal{E} 16$.

\section{FOOTNOTES}

On the web form, you will be able to add footnotes to individual questions, as well as footnotes that apply to your entire institution. Please provide any information which would clarify the figures submitted, e.g., the inclusion of branch campus libraries or any special projects which might cause radical increases or decreases. Please use the footnotes in the ARL Statistics 2007-2008 for comparison if necessary. Please consult the Data Repository under www.arlstatistics.org for a copy of last year's footnotes. These can be found under "Data Repository" after you login to www.arlstatistics.org. Please make an effort to word your footnotes in a manner consistent with notes appearing in the published report, so that the ARL Office can interpret your footnotes correctly. Please use a concise sentence/paragraph format when writing footnotes-do not use bullets or make a bullet list.

NOTE: Any change over 10\% in any answer to any of the survey's questions over the preceding year's response (2007-08) should be addressed with a footnote.

\section{Submit the completed questionnaire by}

October 15, 2009.

For assistance, please e-mail Martha Kyrillidou (martha@arl.org) or Les Bland (les@arl.org)

Tel. (202) 296-2296. 



\section{FOOTNOTES}

Footnotes may also include errata and corrections to data from prior years not previously reported. Numbers refer to columns in Library Data Tables and to Questionnaire numbers. Unless otherwise stated all figures are as of 06/30/2009.

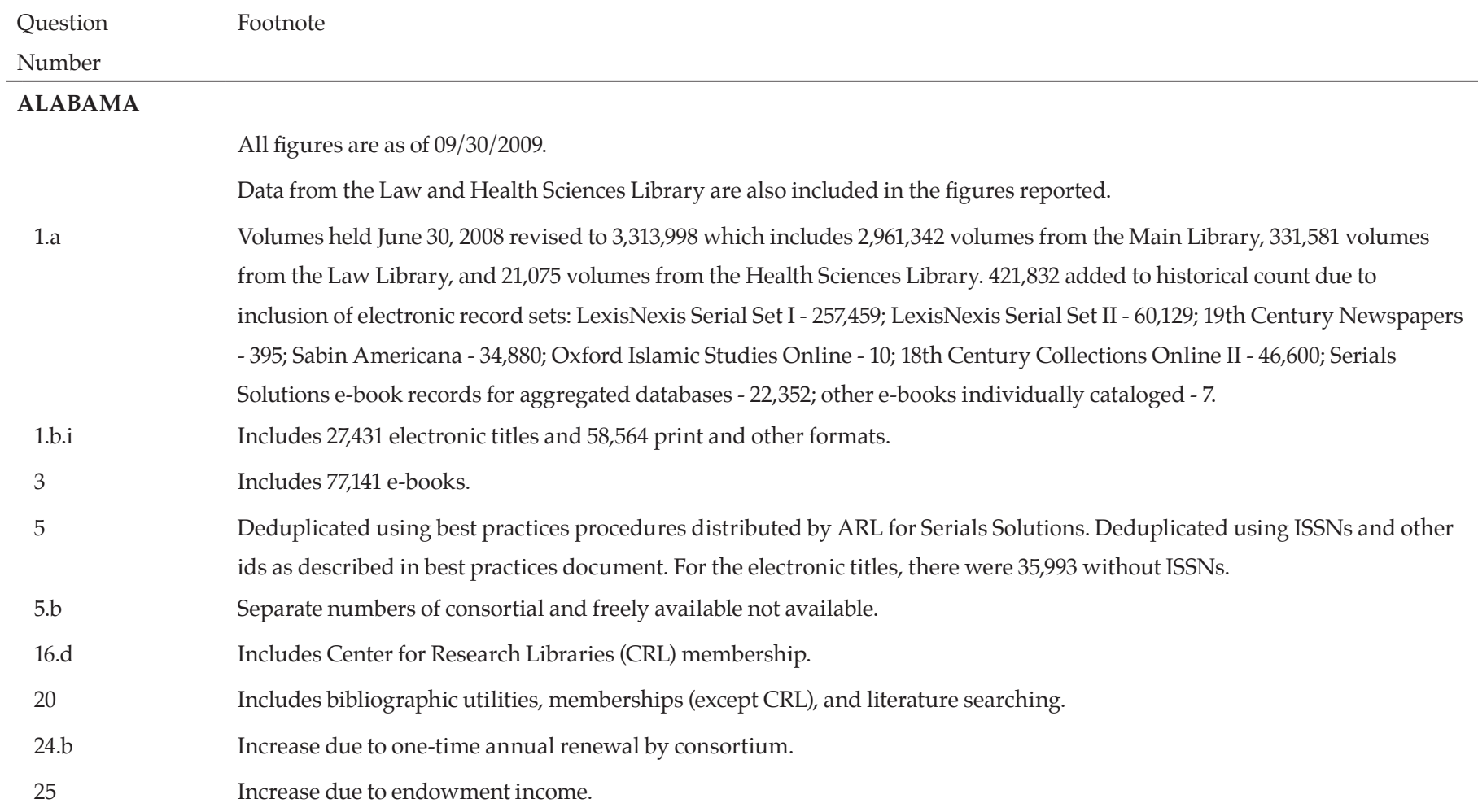

ALBERTA

1

All figures are as of 03/31/2009.

Data from the Law and the Health Sciences Library are also included in the figures reported.

Includes e-book titles.

Includes e-book titles held as of March 2009.

Past figures were inaccurately reported. Precise number of monographs purchased from 2000-2001 onward is unavailable; all monographs purchased are included in gross volumes added (1bi).

This data is included in microform units (7).

Expenditures as reported in Canadian dollars: (16a) \$6,890,560; (16b) \$11,725,718; (16c) 0; (16d) \$939,197; (16) \$19,555,475; (17) $\$ 72,428$; (18a) \$7,742,837; (18b) \$10,601,415; (18c) \$1,267,369; (18) \$19,611,621; (20) \$10,147,115; (21) \$49,386,639; (22) \$7,041,108; (23) \$9,659,123; (24a) 0; (24b) 0; (25) 0; (26) 0 .

Cannot be disaggregated from monograph expenditures (16a) and serial expenditures (16b).

Includes GST (Goods and Services Tax), postage, courier, and supplies expenditures, as well as membership expenditures not already paid through the materials budget.

Figure includes over 5 million dollars of funding for digital library content.

Figure based on actual hours worked. Prior to 2000-2001, figures were based on hours hired to work.

Figure excludes all renewals.

Figure now includes renewals done over the internet; these were not included in years prior to 2007-2008. 
Question Footnote

Number

ALBERTA, cont.

37

38

\section{ARIZONA}

1.b.i

5.a.i

30

32

\section{ARIZONA STATE}

1.a

3

16.a

16.c

16.d

17

18.b

20

22
This figure can vary from year to year.

Number of Ph.D. fields can vary from year to year.

Library branches included: Main, Science Engineering, and Fine Arts.

Data from the Law and Health Sciences Library are also included in the figures reported.

The Library cataloged several large campus/gift collections during 2008-2009.

The figures reported this year represent the deduped serial titles available to the University after the campus-wide implementation of Serials Solutions.

Decrease due to a shift away from classroom style instruction to online instruction.

Decrease due to implementation of online data-gathering procedures. Technological and directional questions were not included (but also decreased).

Library branches included: ASU Tempe, ASU West, ASU Polytechnic, ASU Downtown Phoenix, and the Law Library.

Volumes held June 30, 2008 revised to 4,354,164 which includes 4,079,747 volumes from the Main Library and 274,417 volumes from the Law Library.

A major Tempe approval plan was suspended throughout 2008-2009. Additionally, firm orders and serials binding for all campuses were decreased due to budget uncertainty.

Tempe campus approval plans were suspended throughout the 2008-2009 fiscal year due to budget uncertainty.

Change due to increase in one-time e-book and serial backfile expenditures made late in fiscal year.

Change due to increased membership payment. Additionally, CRL membership was paid from Operations in 2007-2008 but not recorded in $15 \mathrm{~d}$ in error.

Due to budget uncertainty, binding was suspended on all campuses until April 2009.

Less due to FTE Budget Reduction.

OCLC WorldCat Collection Analysis circa were paid from Operations instead of Information budget in 2007-2008, but in 2008-2009, the payment reverted back to Information budget. Decrease in expenditures is also due to temporary budget reductions. Furthermore, funds that were in the past moved to operations from personnel were reverted to accommodate the temporary budget reductions.

Due to uncertainty over budget revertments, major print purchases were suspended until late in the fiscal year. The libraries also identified one-time ER purchases to use money saved from earlier in the year. Money donated from West Operations budget was used to purchase e-books.

Portico payment was reported in Q24a instead of Q23. Furthermore, Amigos was not paid in 2007-2008 due to prepayments in 2006-2007. Additionally, no equipment was purchased in 2008-2009.

See footnote to Q20 (same figure in Q20 and Q25). Furthermore, computer/printer replacement cycle was not done due to budgetary constraints.

2007-2008 figure included almost all of pay-per-view ILL expenses. Furthermore, pay-per-view was not ordered in 2008-2009, and loans exceeded borrowing through Amigos.

Less due to FTE Budget Reduction.

Total student wages were recalculated to reflect average hourly student wage of 7.00 per hour working 1,040 hours per year. Increase due to inclusion of public service hours for all five branch libraries in 2008-2009, whereas the 2007-2008 figure only included only three branches. 
Question Footnote

Number

ARIZONA STATE, cont.

30

31

32

37

41

AUBURN

1, 1.a

1.b

5, 5.b

32

\section{BOSTON}

1.a

\section{BOSTON COLLEGE}

All figures are as of 05/31/2009.

Library branches included: John J Burns Library of Rare Books and Special Collections, Bapst Library, School of Social Work Library, the Educational Resource Center, and the School of Theology and Ministry Library.

Data from the Law Library are also included in the figures reported.

$1 . a$

18.c

37

In 2008-2009, there was less in-person instruction and more instruction via LibGuides and other online resources, especially for large classes e.g. BIO187/188 and WAC \& WR101. Furthermore, the libraries changed their method of counting and are no longer double counting. Additionally, changes in personnel \& librarian vacancies have affected instruction.

Decrease in the number of library presentations to groups resulted in corresponding reduction in number of participants.

Decrease due in part to the reduction of staff to single staffing only and to a change in reference desk location. Further cuts in staffing led to a reduction of reference hours offered. Furthermore, the staffing model for reference help was changed. Reference help is now delivered only on demand. Additionally, decrease due to loss in staff and fewer hours at service desk. Increase due to growth of the University, resulting in more degrees.

Increase due to increased enrollment in existing programs.

All figures are as of 09/30/2009.

Library branches included: Charles Allen Cary Veterinary Medical Library; Library of Architecture, and the Design and Construction Library.

Volumes held revised to 3,434,865. The instructions for Q1b, concerning volumes added during the year, direct us to add any large sets of e-books to the previous year's number of volumes held, and not to count them as volumes added during the year. Auburn University Libraries added the following e-book records during 2008-2009: 312,626 ERIC docs; 54,898 Office of Scientific and Technical Information docs; 9,873 OregonPDF in Health \& Performance (phys. ed. dissertations); 377,397 Total e-books added in these three sets. Adding that total to the number of volumes reported held in 2008-2009 $(3,053,468)$ gives us $3,434,865$ vols.

Reductions in the Libraries material budget, required without the lead time to cut journal subscriptions, led to reductions in purchases of monographs. An additional factor in the decrease of volumes added is the continuing conversion of journal access to electronic format only.

Because of faulty counts in the past several years, Auburn has repeatedly underreported the number of serial titles received, especially in the categories of electronic serial titles currently purchased and in consortial serial titles received but not purchased. The reported numbers for this year are more accurate.

The 2007-2008 figure was misreported; this count is based on a more accurate sample.

Library branches included: Mugar (main), Theology, and Special Collections libraries.

Data from the Law and Medical Library are included in the figures reported.

Volumes held June 30, 2008 revised to 2,847,074 which includes 2,005,866 volumes from the Main Library, 696,009 volumes from the Law Library, and 145,199 volumes from the Medical Library.

Volumes held June 30, 2008 revised to 2,516,668 which includes 2,261,754 volumes from the Main Library and 254,914 volumes from the Law Library.

Decrease due to a change in reporting for a department. This department no longer reports to the library thus the lower amount of student salaries.

Decrease reflects the elimination of Doctor of Education from the count. 
Question Footnote

Number

BRIGHAM YOUNG

All figures are as of 12/31/2008.

Data from the Law Library are included in the figures reported.

1.b The difference in volumes added from the 2007-2008 figure is simply due to fewer e-books cataloged.

3 The difference in monographic volumes purchased from the 2007-2008 figure is simply due to fewer e-books catalogued.

5 The change in distribution of serial titles reflects the change in how these are counted based on the latest instructions for such given by ARL.

\section{BRITISH COLUMBIA}

All figures are as of 03/31/2009.

Library branches included: Applied Sciences Reading Room, Archaeology Reading Room, Architecture Reading Room, Art + Architecture + Planning library, Art History Visual Resources Centre, Asian Library, Biomedical Branch Library, B'nai Brith Reading Room, Botanical Gardens Reading Room, Coolie Verner Reading Room, Crane Library, David Lam Library, Education Library, Geographic Information Centre, Hamber Library, ICICS/Computer Science Reading Room, Irving K. Barber Learning Centre, Koerner Library, Law Library, LERC Reading Room, LLED Digital Literacy Centre, Museum of Anthropology Reading Room, Music Library, Okanagan Library, Rare Books \& Special Collections, Regent College John Richard Allison Library, Robson Square Library, Science \& Engineering Division, St. Mark's College Library, St. Paul's Hospital Library, TAG Reading Room, TRIUMF Library, University Archives, Vancouver School of Theology Library, Womens' Studies Reading Room, Woodward Library, Xwi7xwa Library (First Nations House of Learning).

The Irving K. Barber Learning Centre Phase 2 completed its first full year of operations. UBC Okanagan campus continued to grow.

Volumes held revised to 6,218,261. New base count includes 178,000 e-books.

41,454 e-books were added to the title count of 101,485 volumes.

Increase due to reductions in volumes in reading rooms/affiliated libraries.

Excludes cIRcle, many microform sets, many government publications, maps, graphic, and University Archives-AV.

Count includes physical volumes only.

Unique titles exclude most government publications, serials, and other free serial titles. "Transition to online" program is continuing.

Excludes some government publications, serials, and many titles in microform formats. Reduction in print titles - "Transition to Online" program is continuing.

Some government publications may be included.

Decrease due to change in counting methodology for reporting data files.

New base count for LP's resulted in fewer audio units.

Expenditures as reported in Canadian dollars: (16a) $\$ 4,256,845 ;(16 b) \$ 12,941,503 ;$ (16c) \$0; (16d) \$729,869; (16) $\$ 17,928,217 ;(17)$

$\$ 189,075 ;$; 18 a) \$9,557,328; (18b) \$7,556,960; (18c) \$936,265; (18) \$18,050,553; (20) \$4,579,849; (21) \$40,747,694; (22) \$243,839; (23) $\$ 10,514,793$; (24a) \$983,534; (24b) \$729,869; (25) \$1,148,309; (26) \$115,809.

Additional e-book collections were purchased.

Additional collections of full text serials were purchased.

Backfiles, maps, and audiovisual materials included in Q16a and Q16b.

Figure includes CRKN external funding.

Increase due to contractual salary increases, an increase in the FTE librarian/professional group, and the return of several librarians from study leave (resulting in a change in earnings from $80 \%$ to $100 \%$ salary). 


\begin{tabular}{|c|c|}
\hline $\begin{array}{l}\text { Question } \\
\text { Number }\end{array}$ & Footnote \\
\hline \multicolumn{2}{|c|}{ BRITISH COLUMBIA, cont. } \\
\hline $18 . b$ & $\begin{array}{l}\text { Increase due to contractual salary increases and reclassifications to higher scale as a result of a benchmarking reclassification } \\
\text { process. }\end{array}$ \\
\hline 18.c & Increase due to general increases in salary and coop positions. \\
\hline 20 & $\begin{array}{l}\text { Includes new computers, software, and furniture in IKBLC. Also includes new funding for purchasing hardware/software } \\
\text { for digital initiatives. }\end{array}$ \\
\hline 21 & See footnotes for questions 16.a-16.d, 18.a-18.c, and Q20. \\
\hline 22 & One-time electronic resource purchases figure includes expenditures for e-book collections and backfiles. \\
\hline 23 & $\begin{array}{l}\text { Ongoing electronic resource purchases figure includes expenditures for ongoing serials, fulltext collections, and annual } \\
\text { license fees for e-books. }\end{array}$ \\
\hline 24.a & Increased expenditures: database "walk", added Table of Contents to the catalogue, and added Marc records for e-books. \\
\hline 24.b & Figure includes CRKN external funding. \\
\hline 25 & $\begin{array}{l}\text { Increase due to additional equipment/software for digital initiatives and additional computers in Irving K. Barber Learning } \\
\text { Centre. }\end{array}$ \\
\hline 26 & Decrease due to access to more e-resources. \\
\hline 27.a & Includes librarians and other professional staff at UBC Vancouver, UBC Okanagan, and reading rooms/affiliated libraries. \\
\hline 28 & Excludes reading rooms/affiliated libraries. \\
\hline 39 & 2008-2009 figures were reported for November 2008, instead of November 2007. \\
\hline
\end{tabular}

Library branches included: Rockefeller Library, Sciences Library, John Hay Library, Orwig Music Library, Art Slide Library, Library Collections Annex, and the John Carter Brown Library.

Medical Library statistics cannot be disaggregated from the main because the medical collection is an integral part of the Sciences Library.

Volumes held June 30, 2008 revised to 3,862,067. Volumes held June 30, 2008 reflect the addition of 36,654 e-books. 2007-2008 figure corrected from $\$ 1,934,956$ to $\$ 1,848,033$ (see also “Other library materials”).

2007-2008 figure corrected from $\$ 329,890$ to $\$ 416,813$ (see also “Monographs").

2007-2008 figure corrected from $\$ 169,296$ to $\$ 184,223$.

Up through the 2007-2008 fiscal year, BUL based count of reference questions on sampling technique of the Rockefeller, Sciences, and JCB libraries only, and included both reference and directional transactions. Beginning with the 2008-2009 fiscal year, BUL changed to direct counts of reference activity only (consistent with the ARL definition) and included all libraries.

\section{CALGARY} Increase due to the normal fluctuation in part-time students enrolled.

All figures are as of 03/31/2009.

Library branches included: Health Sciences Library and Law Library.

The fiscal year being reported is April 1, 2008 to March 31, 2009.

Holdings include 334,513 e-books and 136,209 volumes that are part of Eighteenth Century Collections Online. Includes e-book purchases.

$8 \quad$ All government documents are integrated into other counts. 
Question Footnote

Number

CALGARY, cont.

$16-26$

Expenditures as reported in Canadian dollars: (16a) \$3,537,274; (16b) \$8,636,092; (16c) \$630,709; (16d) \$949,860; (16) \$13,753,935;

(17) \$87,271; (18a) \$6,172,855; (18b) \$7,804,344; (18c) \$667,751; (18) \$14,644,950; (20) \$1,283,056; (21) \$29,769,212; (22) \$921,988; (23)

$\$ 6,921,974 ;$; (24a) \$284,891; (24b) \$307,867; (25) \$401,260; (26) \$122,163.

29

Information Commons is open $24 / 7$ during session.

\section{CALIFORNIA, BERKELEY}

Data from the Law Library and from Affiliated Libraries are included in the figures reported.

1.a

Volumes held June 30, 2008 revised to 10,884,802 which includes 9,233,669 volumes from the Main Library, 693,983 volumes from the Law Library, and 957,150 volumes from Affiliated Libraries.

\section{CALIFORNIA, DAVIS}

Library branches included: Shields Library, Physical Science and Engineering Library.

Data from the Law Library and the Health Science Libraries are included in the figures reported.

1

Includes 393,564 e-books previously unreported.

1.a

Volumes held June 30, 2008 revised to 4,074,855 which includes 3,406,958 volumes from the Main Library, 297,061 volumes from the Law Library, and 370,836 volumes from the two Health Science Libraries. Figure includes 393,564 e-books previously unreported.

5

Reflects the 2009 change in methodology for counting serials titles by the California Digital Library from catalog-based to SFX-based. See http://libraries.universityofcalifornia.edu/planning/stats/index.html

5.a, 5.b Figure reflects corrected counting mechanism.

14 Increase due to the transfer of a large collection to the library from another department.

16.b No longer separating Other and Miscellaneous.

18.c, 27.c Decrease due to budget reduction.

27.b Decrease due to decision to deliberately leave positions unfilled.

41, 43 Figure reflects corrected information from Student Affairs Research and Information office.

\section{CALIFORNIA, IRVINE}

Data from the Health Science Library are also included in the figures reported.

\section{CALIFORNIA, LOS ANGELES}

Data from the Law and Biomedical Library are also included in the figures reported.

1.a

Volumes held June 30, 2008 revised to 8,926,378 which includes 7,695,647 volumes from the Main Library, 565,343 volumes from the Law Library, and 665,388 volumes from the Biomedical Library. Figure adjusted to include 532,468 e-books.

5 Data includes all units; Law and Health Sciences. 2007-2008 figure included subscriptions. 2008-2009 figure is unique titles only.

Decreased computer expenditures due to a reduction in computer hardware purchases. There was a much smaller decrease in computer software expenditures, partly offset by increased expenditures for computer maintenance. Computer hardware expenditures decreased partly due to a decision to increase the length of the workstation replacement cycle.

\section{CALIFORNIA, RIVERSIDE}

1.a

1.b.ii
Volumes held June 30, 2008 revised to 2,908,583. UCR Libraries have not previously reported e-books that were already included in the catalog. This figure includes 380,976 e-books, listed as follows: UC System-wide Tier 1 e-books = 359,105; Local Tier 2 e-books: 18,103; University of California Press eScholarship editions: 1,845.

This figure includes only the volumes withdrawn and discarded, not those volumes withdrawn in lieu of deposit in the UC Southern Regional Depository which supports the libraries of all of the southern UC campuses. 
Question Footnote

Number

CALIFORNIA, RIVERSIDE, cont.

3

5.b.i

5.b.ii

5.b.iii

14

16.d

17

\section{CALIFORNIA, SAN DIEGO}

Library branches included: Arts Library, CLICS: Center for Library and Instruction Computing Services, East Asia Collection, International Relations and Pacific Studies Library, Mandeville Special Collections Library, Science and Engineering Library, Scripps Institute of Oceanography Library, Social Science and Humanities Library.

Data from the Health Science Libraries are included in the figures reported.

1.a

3

16.c

18-18.c

28

32

\section{CALIFORNIA, SANTA BARBARA}

Library branches included: Arts Library.

18-18.c Includes fringe benefits.

\section{CASE WESTERN RESERVE}

Library branches included: The Mandel School of Applied Social Sciences - Harris Library.

Data from the Law and Health Science Library are included in the figures reported.

16.d

This expenditure includes memberships for ARL, SPARC, CNI, OhioLINK, ICPSR, and EEBO/TCP and theses charges from ProQuest.

38

Decrease due to streamlining of the way we count programs for purposes of our accrediting agency.

\section{CHICAGO}

1

1.b, 1.b.ii

2
Library branches included: Law and Health Sciences Libraries.

Includes 831,860 electronic monographs.

Includes 5,747 previously uncounted withdrawals.

Includes 35,414 electronic monographs purchased this year; does not include monographs for which we previously had access and had counted as added, but added MARC records for this year. 
Question Footnote

Number

CHICAGO, cont.

3

5.a.i

5.a.ii--5.b.iv

9-14

22

23

24.a

24.b

36

\section{CINCINNATI}

$1 . a$

4

5

8

32.a

37

\section{COLORADO}

1.a
Fewer electronic monographs were purchased.

The increase in this category over 2007-2008 reflects a thorough recounting of paid electronic subscriptions and changes to the deduping procedure.

Includes switching many subscriptions to online-only from print subscriptions. Figure also reflects a more accurate deduping and counting of free titles.

Excludes unprocessed and partially processed items.

Reflects reduced budget for one-time purchases.

Includes switching many subscriptions to online-only from print subscriptions. Figure also reflects a more accurate deduping and counting of free titles.

Includes standard ongoing operating costs only. Also, credits used for payment have been incorporated.

This figure is an estimate.

Includes 443 requests filled by the Center for Research Libraries.

Library branches included: all University of Cincinnati Libraries located on its central campus.

Two branch libraries on two-year college campuses, Raymond Walters College and Clermont College, are not included in the ARL statistics.

Data from the Law and Health Sciences Library are also included in the figures reported.

Volumes held June 30, 2008 revised to 3,695,648 which includes 3,103,861 volumes from the Main Library, 288,947 volumes from the Law Library, and 302,840 volumes from the Health Sciences Library. This figure reflects net adjustments plus the addition of 63,991 volumes, which include the following: a 1,539 Net Government Documents adjustment (1,931 volumes added for retro cataloging minus 392 volumes subtracted for retro cleanup); 0 volumes added for other retro; 0 volumes subtracted for other retro; and 62,452 volumes added for batch load electronic resources.

Historical is based on bibliographic count. Additions and withdraws based on physical count.

The serial count is by title, deduped, in a "cascading hierarchy". (1)Paid (first electronic, then other formats) - A large majority of the number of serial titles received via our consortium are paid via index figures based upon titles owned when the relationships with the publishers began. Therefore, all are considered Paid. (2)The Not-Purchased titles in the Freelyaccessible and Government documents categories have very little overlap, because of the strategy used to construct the serials list, and then were deduped; however, numbers here are only the titles that have not already been included in the Paid category. (3)The Freely accessible titles include the titles marked as "free"? from Serials Solutions, so long as those same titles had not already been recorded among the paid Serials Solutions titles. (4)Some Government document titles are also included in Paid Serials Solutions aggregates, and not-purchased Government document titles could also be included in the free Serials Solutions titles.

Government documents not counted elsewhere is smaller than the number reported for 2007-2008 because of retrospective cataloging of documents.

Main and Law reference transactions figures based on sampling. Med figures based on physical count.

For questions 37 through 43: PhD degrees, faculty, and enrollment numbers reported here are the total university counts.

Library branches included: Business, Earth Sciences, Engineering, Math/Physics, and Music.

Volumes held June 30, 2008 revised to 4,274,324. This number has been adjusted to reflect the 2008-2009 addition (via batch loading) of 317,573 Congressional Serial Set Records; 6,983 other free online resources; and 21,337 purchased online resources (including 16,054 Springer e-books). 
Question Footnote

Number

COLORADO, cont.

1.b.ii Our withdrawal numbers are much lower because we've withdrawn a large number of volumes of serials in past years and now have gone to buying many more electronic materials, thus reducing our withdrawal figures considerably.

3

5.a.ii

5.b.iii

16.d

17

\section{COLORADO STATE}

1.a

1.b.i

3

5

\section{COLUMBIA}

1
Includes 27,849 batchloaded e-books.

We dropped a few state-funded databases where we would have counted print subscriptions as electronic in 2007-2008.

In 2007-2008, we counted only titles with gift or exchange order records; this year we also counted titles with check-in records with "gift" noted.

This consists of: electronic archiving $(\$ 9,750)$, preservation (not incl. contract binding - $\$ 62,629)$, other preservation $(\$ 11,140)$,

GPO shelflist service (\$4,636), III services $(\$ 111,420)$, interlibrary loan $(\$ 31,715)$, MARC record batches $(\$ 21,058)$, memberships $(\$ 301,100)$, BCR $(\$ 206,525)$, OPAC enhancements $(\$ 52,250)$, storage (Boulder $\$ 15,792)$, Prospecter $(\$ 5,500)$, shelf-ready processing (\$89,114), and Backstage backfile $(\$ 50,000)$.

This year we're using the figure for contract binding from our preservation librarian, which is a lower but more precise figure for what ARL asks to include in this question. Acquisitions has provided the figure in the past, but their figure includes other items paid to the commercial binding vendor than just contract binding and, thus, has always been a larger figure.

We spent $\$ 515,125$ on this, but it does not come from the Operating Expenditures per the instructions, so we're putting 0 here.

Lower figure because we purchased only 5 (versus about 60) replacement computers during 2008-2009 (not including the faculty computer replacement program). This was due to a lack of staff to order/install/maintain computers. We also purchased about $\$ 10,000$ less in software.

Volumes held June 30, 2008 revised to 2,288,232.

New methodology now allows the library to count cataloged e-docs as part of the volumes held.

Increase due to purchase of large e-book sets during the 2008-2009 fiscal year.

Increase due to purchase of electronic serials no longer provided by state contract.

Additional funding transferred from University for purchase of Web of Science previously included in external source question.

Increase due to inflation costs and move of costs from University to Library for purchase of Web of Science database.

All figures are as of 08/31/2009 [Teachers College].

Columbia data includes The Columbia Center for New Media Teaching and Learning (CCNMTL) and the Center for Digital Research and Scholarship (CDRS.) Data from the Barnard, Butler, Law, Health Sciences, and Teachers College Library are included in the figures reported.

Includes 733,655 e-books. [Butler]

Includes 72,700 e-books. [Teachers College]

Performing inventory which included sizable withdrawal and donation of college catalogs for digitization to CollegeSource. [Teachers College]

Barnard serials data included in Butler Library statistics. [Barnard]

Reflects unique titles; all other titles received by Teachers College overlap with, and are counted in, Butler Library statistics. [Teachers College]

This figure is an estimate. We are doing a major inventory. [Teachers College]

Includes costs associated with maintenance of collection (e.g., shipping, supplies, relocation, memberships). [Butler] 
Question Footnote

Number

COLUMBIA, cont.

26

26

35

\section{CONNECTICUT}

$1,1 . b$

1.a

3

5

5.b.iv

16,22

18-18.c

18.c

20,25

23

26

30

36

37

40

41

42

43

CORNELL
Document Delivery / Interlibrary Loan figures included in Butler Library statistics. [Barnard]

Document Delivery / Interlibrary Loan figures included in Butler Library statistics. [Teachers College]

ILL for Barnard Library handled by Butler office. [Barnard]

Library branches included: Music, Art, Pharmacy, Avery Point, Waterbury, Stamford, Torrington and Greater Hartford.

Data from the Law and Health Science Library are also included in the figures reported.

Main Library completed/ceased the project to reduce materials no longer relevant to the institutions current curriculum which resulted in an overall increase in volumes held and fewer volumes withdrawn. This action impacts results for all answers in Q1.

Volumes held June 30, 2008 revised to 3,978,619 which includes 3,432,126 volumes from the Main Library, 349,318 volumes from the Law Library, and 197,175 volumes from the Health Science Library. Figure reflects the addition of 59,196 records for Making of the Modern World, an e-book package acquired via one-time download.

Figure reflects decreased emphasis in print monograph collecting for Main Library.

Main Library has decreased emphasis on print collecting, funds diverted to collecting additional e-content. Two additional, significant changes occurred: 1) Lexis Nexis Academic reverted to a purchased e-resource from a non-purchased consortial (consortia canceled the subscription); and 2) IEEE ASPP upgraded to IEEE/IEL Electronic Library for Main Library. Also a more accurate count of government document tiles is reflected in this figure.

Increased effort to accurately reflect government document titles in our statistics.

Main Library expended some residual funds on materials for 2008-2009. The majority of these funds were directed toward increasing available e-content and continuing and one-time services in all formats.

Includes fringe benefits.

Main Library student assistant figure is gross FTE.

Main Library received less equipment budget funding from University than in previous fiscal year.

Main Library has increased emphasis on collecting e-content. Main Library expended some residual funds on materials for 2008-2009. The majority of these funds were directed toward increasing available e-content and continuing and one-time services in all formats.

Increased demand for materials not held internally led to higher DD/ILL costs.

In an effort to serve all users effectively, Main Library has increased number of participants in sessions offered and decreased number of sessions available.

Figure reflects increased demand for materials not held internally.

2007-2008 figure needs to be revised to 285 .

2007-2008 figure needs to be revised to 19,926 .

2007-2008 figure needs to be revised to 3,766.

2007-2008 figure needs to be revised to 4,311 .

2007-2008 figure needs to be revised to 3,033.

Library branches included: The Adelson, Africana, Annex, Asia Collections, Engineering, Fine Arts, Hotel, Industrial \& Labor Relations, Kroch (Rare \& Mss. Collections), Law, Management, Mann/Entomology, Mathematics, Music, Olin, Physical Sciences, Uris, and Veterinary Libraries on the Ithaca campus, and the New York Agricultural Experiment Station Library in Geneva NY.

Library branches NOT included: The Medical College Library in New York, NY. 
CORNELL, cont.

This year, CUL switched from a manual to an online physical volume count. This new count is not directly comparable to previous years' counts as it excludes any volumes that have not been bar coded, or that accompany non-volume format items, but includes locally held volumes stored digitally. The count also includes some e-book growth, and the addition of a large number of catalog records for e-books already owned/subscribed. E-book count includes some duplication between e-book packages yet to be determined.

This figure may be high due to the decision to switch to online counts during the fiscal year. It is likely that not all bar coding done retrospectively was removed. Includes a few Law e-books cataloged manually.

The e-book count included has not been deduplicated with the print count. The e-book count also includes some duplication between e-book packages yet to be determined. We are currently looking into how deduplication might be accomplished.

Estimate only. $27.6 \%$ of the titles were estimated to be print. 9,000 of the e-titles were estimated to be non-current. This count includes two Law aggregators that do not have individual records in the online catalog as they are only available to Law users. Deduping was done through estimates, some of which were based on sampling. Following ABA definitions, the Law print count includes non-serial continuations. The decrease in the print count is due to cancellations to favor electronic format over print, and a more precise counting methodology. The increase in the e-title count is due to both the availability of new titles, and, mostly, to an increase in the availability of online records for previously purchased or licensed titles. Starting with 2008-2009, microform counts will no longer be kept up to date; reporting will be at the 2007-2008 level. The change reflects rounding only.

Decrease reflects a change from a manual to an online count that excludes any computer files that accompany other formats, and/or that are not bar coded.

Figure is in cubic feet.

Starting with 2008-2009, map counts will no longer be kept up to date; reporting will be at the 2007-2008 level. The change reflects rounding only.

Starting with 2008-2009, graphics counts will no longer be kept up to date; reporting will be at the 2007-2008 level. The change reflects rounding only.

Decrease reflects a change from a manual to an online count. Excludes any sound recordings that are not bar coded and/or that accompany other formats.

Starting with 2008-2009, motion picture counts will no longer be kept up to date; reporting will be at the 2007-2008 level. Video and DVD counts are now collected online, not manually.

This year, this figure includes expenditures for integrating resources as well as shipping costs not included in 16a-c.

Previously, expenditures for items coded as integrating resources were reported in 16a. Per cataloging rules, more and more items will be cataloged or recataloged (from other formats including monographs and serials) as integrating resources. These expenditures currently include those for such items as reference databases, resource aggregators (including those with a mix of formats), etc.

Includes expenditures for librarians and archivists only.

Expenditures include some in-year returns on reserves mandated due to the recession.

Includes an estimate for the amount spent on electronic subscriptions for large publishers providing both print and electronic whose payment records are coded as print only. In 2008-2009, payment was made for 2007-2008 and 2008-2009 for ILLiad.

Does not include staff in positions that were temporarily vacant. Includes professional, support, and student staff on grants and projects.

Includes librarians and archivists only. 
Question Footnote

Number

CORNELL, cont.

28

29

30

32

33

36

41,43

DARTMOUTH

1.b.i

1.b.ii

3

5.a.ii

14

16.c

17

20

22

23

26

35

41,43

\section{DELAWARE}

DUKE
Decrease reflects the discontinuation of 3 service points, as well as some corrections to reporting practices; we are now counting combined service points by designated areas and not by function (down 4), and are now viewing chat service as a mode of contacting the reference desks and not as separate service points (down 3).

Part of one library remains open between 2 and 8 a.m., Sunday-Thursday, staffed by library-paid security staff (which adds 27.5 hours to this figure). Study space and computer lab available.

Includes some instruction sessions for individuals, and some duplication for co-taught sessions.

The 2007-2008 figure should have been 71,882. Most units' counts are extrapolated from 12 random sampling weeks.

Includes ILL lending and some staff transactions.

Borrow Direct counts are volume counts; traditional ILL counts are title counts. Traditional ILL counts include some of the requests submitted by CUL patrons for items that are available at CUL.

Employee degree program only.

Library branches included: Baker-Berry Library, Feldberg Business \& Engineering Library, Kresge Physical Sciences Library, Paddock Music Library, Sherman Art Library, Rauner Special Collections Library and College Archives, and the Storage Library.

Data from the Health Sciences Libraries are also included in the figures reported.

E-books were not included in the 2007-2008 count.

Withdrawals part of ongoing deaccessioning project.

E-books were not included in the 2007-2008 count.

Decrease due to conversion to electronic only.

Figure reflects response to programmatic growth.

The increase in spending correlates to our increasing film and video collection.

Reduction due to budget constraints and decaccessioning.

Increase in Other Operating Expenditures is due to a significant increase in endowment distribution (50\%) in 2008-2009 and increased reserve spending during the year.

Figure indicates long-term planned purchases.

Figure indicates emphasis on electronic only.

Figure correlates to expansion of electronic holdings.

Figure indicates increased consortial activity.

Part-time graduate students vary from year to year as non-degree participants are counted in this figure (employees, community members).

Includes 290,322 e-books.

Does not include 290,322 e-books.

Excludes the University Archives that is not administered by the Library.

Library's automated system does not differentiate between initial loans and renewals.

Library branches included: Perkins Library (Main Library), Music Library, Lilly Library, Rare Books Manuscripts Special

Collections Library, and Marine Lab Library.

Data from the Law and Health Science Library are also included in the figures reported. 
Question Footnote

Number

DUKE, cont.

1.a

1.b.ii

5, 5.a-5.b

9

16.a

16.c

22

25

41,43

\section{EMORY}

1.a

1.b

1.b.ii

1.b.ii

2

3

5

5.a

5.a.i

5.a.ii

5.b

$5 . b$

5.b.ii

9

13

14
Volumes held June 30, 2008 revised to 5,945,226 which includes 5,169,368 volumes from the Main Library, 475,553 volumes from the Law Library, and 300,305 volumes from the Health Science Library.

Increase due to the Medical Center Library withdrawing over 50,000 volumes from their collection.

Increase due to the library including many titles that were excluded previously, due to better data gathering methods this year.

Decrease due to reporting some Computer Files as Digital Collections this year, in Sup. no. 10.

Decrease apparent because in past fiscal years Strategic Initiative Funding supplemented the Collections Budget. This funding has decreased in 2008-2009.

Increase due to increased expenditures in this area.

Decrease apparent because in past fiscal years Strategic Initiative Funding supplemented the Collections Budget. This funding has decreased in 2008-2009.

Decrease apparent because in past fiscal years hardware was refreshed for all library staff and for library patrons during renovations. This hardware replacement cycle did not occur to the same degree in 2008-2009.

These numbers fluctuate annually based on normal enrollment fluctuation in various academic departments.

All figures are as of 08/31/2009.

Library branches included: The Health Sciences Center, Hugh MacMillan (Law), Oxford College, and Pitts Theology libraries, as well as libraries at Grady Hospital and the Medical School [Main Library].

Volumes held June 30, 2008 revised to 3,399,555 which includes 2,270,032 volumes from the Main Library, 90,547 volumes from the Oxford Library, 553,575 volumes from the Theology Library, 245,073 volumes from the Law Library, and 240,328 volumes from Health Science Libraries. [Main Library]

Figure reflects improved selection process for acquisitions. [Oxford College Library]

Increase due to greater withdrawal of print copies where electronic format is available and weeding project to gain space. [Main Library]

Heavy weeding of reference collection occurred in previous year. [Oxford College Library]

E-book titles not deduped. [Main Library]

Figure reflects improved selection process for acquisitions. [Oxford College Library]

Emory \& the Galileo consortium purchased a number of e-journal packages in 2008-2009, e.g. Freedom Collection and 9,819

"free" e-journals. We over-reported “free" titles in 2007-2008 as 11,795. Also, we may have under-reported some consortial serials in 2007-2008. [Main Library]

Based on SFX report, number of serial titles currently purchased includes GALILEO \& GETSM agreement titles. [Main Library]

2008 count incorrectly reported online plus print. [Oxford College Library]

Decrease due to move from print to electronic. [Main Library]

Free titles in SFX not split with other Emory libraries in 2007-2008. [Main Library]

Counted school publications received from Emory. [Oxford College Library]

16,871 free titles in SFX split this year with Health Sciences, but not split in 2007-2008. Figure does not include GovDocs.

[Main Library]

Figure reflects increased purchases for this year. [Oxford College Library]

Figure reflects increased requests for CDs. [Oxford College Library]

Figure reflects increased requests for DVDs and heavily collecting in this popular area. [Oxford College Library] 
EMORY, cont.

16.a

16.b

16.c

17

18.a

18.c

22

24.b

25

26

28

31

32

\section{FLORIDA}

5.b.ii

39

FLORIDA STATE

1.a

1.b

1.b.i

5.a.i

5.b.i

5.b.ii

10
Shifting percentages due to move from print to electronic and purchase of many backfiles. [Main Library]

Figure reflects the cancellation of subscriptions to reduce costs. [Oxford College Library]

Figure reflects increased overall purchases. [Oxford College Library]

Only sent one shipment to the bindery this year. [Oxford College Library]

Figure reflects reductions of library staff due to economic conditions. [Oxford College Library]

Figure reflects increased number of library student employees. [Oxford College Library]

Increase due to substantial one-time e-resource purchases for general collections and MARBL (special collections). [Main

Library]

Increased cataloging due to increased purchases. [Oxford College Library]

Decreased purchases due to economic conditions. [Oxford College Library]

Increase likely caused by increased accessibility to ILL option through new library interface, DiscoverE. [Oxford College Library]

Figure reflects reduced service desks. [Main Library]

Fewer staff are providing instruction sessions, but there are a greater number of larger sessions. [Main Library]

The 2007-2008 figure should have been 14,032. Data for 2008-2009 are incomplete. [Main Library]

Library branches included: All Gainesville, Florida campus libraries: Marston (Science), Architecture/Fine Arts, Journalism, Music, Education, Law, and Health Science Center. Also includes the Health Science library facility in Jacksonville, Florida.

This number increased because we started using a new Serials Solution feature that provides access from our catalog to available open access journals.

This number is the same as reported for 2008; the statistics for 2009 will not be updated by the University of Florida until February 2010.

Library branches included: Main Library (Strozier), Dirac Science Library, College of Engineering Library, Allen Music Library, Goldstein College of Information Library, Ringling Museum of Art Library, Panama City, Panama Library, and Panama City, Florida Library.

Data from the Law and Health Science Library are also included in the figures reported.

Volumes held June 30, 2008 revised to 3,030,833 which includes 2,667,976 volumes from the Main Library, 354,161 volumes from the Law Library, and 8,696 volumes from the Health Science Library.

Volumes added - net. decrease in volumes added due to budget cuts. University Libraries (Main, Dirac Science and Engineering) approval plan dropped many titles and every library at FSU experienced budget cuts this past fiscal year. All counts (except for College of Medicine) were down. There was also an increase in volumes withdrawn due to several special weeding projects at the Main Library, principally a project to withdraw second copies and the withdrawal of non-Florida State documents.

Increase due to addition of a 3,532 volumes of bound serials previously owned but not counted.

Increase due to the acquisition of Academic Search Complete which added 7,422 additional journals.

The increase in the number of Consortial journals went up due to the acquisition of new databases.

The number of freely accessible journals went up because we activated two freely accessible databases within Serials Solutions.

There was a decrease in this figure because the Claude Pepper Center Library's holdings were not included in this year's count. 
Question Footnote

Number

FLORIDA STATE, cont.

24.a Ringling Library's figure $(\$ 77,000)$ now includes OCLC charges and fees that were not included in the 2007-2008 figure.

29

32

35

\section{GEORGE WASHINGTON}

Library branches included: The Gelman Library System includes the Main University Library and libraries at the Mount Vernon and Ashburn campuses.

Data from the Law and Health Sciences Library are also included in the figures reported.

$1 . a$

$1 . b$

1.b.i

5.b.ii

5.b.iv

10

13

14

16.b

16.c

16.d

17

18.b

18.c

20

25

Volumes held June 30, 2008 revised to 2,238,927 which includes 1,746,125 volumes from the Main Library, 362,303 volumes from the Law Library, and 130,499 volumes from the Health Science Library.

In 2007-2008, we conducted a major weeding project. There was not a similar project conducted in 2008-2009.

Added a collection of International Business books donated by the World Bank.

We are cataloging these titles differently this year than in previous years.

In previous years we counted these items with monograph government documents. This count is just the government documents that are serials.

Special Collections acquired the National Education Association collection, an additional 2,661 linear feet.

We purchased more audio titles in 2008-2009.

We purchased the entire Criterion Collection (450) to add to our Media collection.

This is just the monographic count for 2008-2009. In 2007-2008, we combined with Electronic expenditures.

In 2007-2008, we paid a one-time fee and more backfiles were purchased.

WRLC membership fees increased.

Because of changes in our binding policy and the switch to online preferred, we bound far fewer serials in 2009.

A number of positions were re-purposed into base salaries in order to improve recruitment efforts and increased parity in the marketplace

A hiring freeze was initiated in December specifically focused on these positions.

Some of our additional operating expenditures for this are the purchase of Aquabrowser, additional computers and computer services and the National Education Association project.

We purchased Aquabrowser in 2008-2009.

Includes 3 non-MLS professional staff. There are currently 4 open positions still to be filled.

A number of positions were re-purposed into base salaries in order to improve recruitment efforts and increased parity in the marketplace.

A hiring freeze was initiated in December specifically focused on these positions.

Due to further consolidation of services, service desks have been eliminated and the count in the 2007-2008 fiscal year was inaccurate.

The Education and Instruction Group continue to expand their program. New collaborations with professors from History and Physics contributed to the increased numbers in instruction sessions. Another factor in this increase was the inclusion of distance education courses in the total number of sessions. This resulted from further involvement in the Education and Instruction Group in instructional efforts at our satellite campuses. The increase in presentations is also due to the hiring of a Public Services and Outreach Librarian for Special Collections. 
Question Footnote

Number

GEORGE WASHINGTON, cont.

32.a

37

\section{GEORGETOWN}

1.a

36

\section{GEORGIA}

1

$16 . d$

\section{GEORGIA TECH}

1.b.i

5.a

14

17

24.a

25

32

34

\section{GUELPH}

1.b.i

1.b.ii

5.a.i

5.b.ii

$16-26$

16.b

16.c

16.d
Two locations report sampling. One is a sample of a period of time, 3 weeks between February and March. The other is an estimate of 20 transactions per week for 40 weeks.

The School for Engineering and Applied Sciences is now awarding PhD's instead of DSc's.

Data from the Law and Health Science Library are also included in the figures reported.

Volumes held June 30, 2008 revised to 3,371,878 which includes 2,359,424 volumes from the Main Library, 912,482 volumes from the Law Library, and 99,972 volumes from the Health Science Library.

Increase due to ArticleReach statistics being included in the 2008-2009 figure.

Data from the Law Library are also included in the figures reported.

Includes OECD (3,045), PAHO (12), EEBO (164,896), Gale (10), Misc. e-books (42).

Includes 199 e-books purchased.

Memberships \$92,531; Bibliographic utilities \$100,992; Shipping \& Handling \$59,851; Endnote \& Refworks \$40,702; Media $\$ 50,727$; Maintenance $\$ 1,500$.

Library branches included: Main Library and the Architecture Library.

Discontinued binding print journals for cost savings. This significantly reduced the number of volumes added.

Modified method of counting journals and reduced number of subscriptions. This reduced the overall journal count.

Purchased large number of DVDs with grant funding.

Discontinued binding journals during 2008-2009 for cost savings.

We made a large deposit to our deposit account at the end of 2007-2008. We deferred making significant payments in 20082009 for cost savings.

Decrease due to deferment of staff computer purchases.

We began including reference statistics from our branch library in 2008-2009. Figure reflects this change.

This should have been reported as unavailable in 2007-2008. We do not count renewals.

All figures are as of 04/30/2009.

Library branches included: McLaughlin Library (Main), Ontario Veterinary College Library.

Includes both print and electronic monograph volumes, unlike 2007-2008.

Variation in volumes withdrawn, relative to 2007-2008, not seen as significant. Space for collection growth is limited.

Includes total number of electronic serial titles available through aggregator packages, accessible through Library web site.

Total now includes the journals listed in the Directory of Open Access Journals (4,375 in total) available through Library's web site.

Expenditures as reported in Canadian dollars: (16a) \$1,353,742; (16b) \$4,295,728; (16c) \$184,497; (16d) \$954,570; (16) \$6,788,537; (17) $\$ 27,844$; (18a) $\$ 3,368,440 ;(18 b) \$ 3,030,466$; (18c) \$259,780; (18) \$6,658,686; (20) \$1,103,702; (21) \$14,578,769; $(22) \$ 425,818 ;(23)$ $\$ 4,061,661$; (24a) \$206,270; (24b) \$284,051; (25) \$469,228; (26) \$91,408.

Serial expenditures increased due to funding increases and inflation.

Increase in expenditures due to one-time purchase of electronic products like journal backfiles.

Marked increase due to "infrastructure" payments to support OCUL Scholars Portal, Canadian Research Knowledge Network, open access, and other initiatives. 


Question Footnote

Number

\section{GUELPH, cont.}

22

23

HARVARD

1.a

\section{HAWAII}

1.b.i

1.b.ii

3

5.b.ii

16.a

$16 . b$

17

18.a-18.b

20

22

23

25

26

29

30-31

\section{HOUSTON}

2

16.c, 22

25

26
Increase in expenditures due to one-time purchase of electronic products like journal backfiles.

Serial expenditures increased due to funding increases and inflation.

Data from the Law and Health Science Library are also included in the figures reported.

Volumes held June 30, 2008 revised to 16,254,755 which includes 13,779,615 volumes from the Main Library, 1,788,345 volumes from the Law Library, and 686,795 volumes from the Health Science Library.

Library branches included: University of Hawaii at Manoa Library (main) housed in two buildings: Hamilton Library, and the Sinclair Library.

Data from the Law and Health Sciences Library are also included in the figures reported.

Many materials purchased in 2007-2008 using flood recovery funding were processed in 2008-2009.

Efforts increased in 2008-2009 to deaccession materials in order to gain space for new acquisitions.

Approval plan expanded following reduction in 2007-2008.

Additional titles added to electronic resources gateway.

Flood recovery funding from 2007-2008 did not continue into 2008-2009. Some prepayment in previous year for approval plan receipts in 2008-2009.

Supplemental budget funding received to purchase electronic resources such as JSTOR collections and ArtSTOR.

Increase in number of serial volumes bound in 2008-2009.

Increase due to negotiated salary increases.

Funding for roof leak repairs increased operating expenditures in 2007-2008. Some major expenses were encumbered but not expended in 2008-2009. In addition, there was some reduction in operating costs, travel, and innovation funding in 20082009.

Supplemental budget funding in 2008-2009.

New electronic resources were purchased with supplemental budget funding, including one year of maintenance fees.

Supplemental budget funding in 2008-2009.

Expenditures for document delivery and interlibrary borrowing of books continue to increase due to journal cancellations and growing gaps in monographic holdings.

Increase due to $24 / 7$ hours of operation begun in one library building.

Increase due to expanded instruction program for freshman composition courses.

All figures are as of 08/31/2009.

Library branches included: Architecture \& Art Library, Music Library, Weston A. Pettey Optometry Library, Pharmacy Library and the Law Library.

Titles include 1,629,798 print titles and 388,743 titles of electronic materials which includes 51,913 US Government Documents titles.

The library purchased ProQuest this year and charges for this service have been recorded here. This accounts for the increase.

The library expanded its learning commons, bought additional storage and new software such as Encore and Serials Solutions.

Decrease in this expenditure is proportional to decreased interlibrary loan activity. 
Question Footnote

Number

HOUSTON, cont.

The current organizational chart, list of staff members, and university human resource records were thoroughly checked to verify correctness of this figure.

\section{HOWARD}

1.a

Data from the Law and Health Science Library are also included in the figures reported. Lines 10, 12, 13 are new counts. Volumes held June 30, 2008 revised to 2,570,927 which includes 2,226,202 volumes from the Main Library, 210,717 volumes from the Law Library, and 134,008 volumes from the Health Science Library.

\section{ILLINOIS, CHICAGO}

Library branches included: Richard J. Daley Library and the Science Library.

Data from the Health Sciences Library are also included in the figures reported.

1.a

Volumes held June 30, 2008 revised to 2,455,685 which includes 1,714,210 volumes from the Main and 741,475 volumes from the Health Sciences Library.

3

Number of monographic volumes purchased for the Richard J. Daley Library estimated based on 2007-2008 ARL Statistics data. The total number of monographic titles including large one time purchases of e-books purchases between 2006 and 2009 is 223,699 monographic volumes.

Figure reported reflects Health Sciences Library only. Main library contract binding expenditures included in other operating expenditures.

\section{ILLINOIS, URBANA}

1.a

1.b.i

\section{INDIANA}

1

1.a

1.b

3

5, 5.a.i

5.b.ii

8

Data from the Law Library are also included in the figures reported.

Volumes held June 30, 2008 revised to 12,518,870 which includes 11,894,429 volumes from the Main Library and 624,441 volumes from the Law Library.

Increases in monographic holdings attributed, in part, to significant investments in retrospective cataloging aimed at addressing long-term backlogs in processing.

58,113 e-books included in this total.

Library branches included: Wells Library.

Library branches NOT included: Medical Library.

Data from the Law Library are also included in the figures reported.

Volumes added to the volume count: 122,460; Collections: Congressional serial set, MIT Cognet, Sabin Americana, US Depository e-books. E-book holdings adjustments to 2007-2008 holdings from under and over reporting in previous years. Making of Modern Law, HeinOnline World Trials, and other resource title adjustments were made. OPIE Collection of Children's Literature was removed as it is a microform collection for which we loaded bibliographic records. Total increased to 45,076 .

Volumes held June 30, 2008 revised to 8,407,231 which includes 7,916,144 volumes from the Main Library and 491,087 volumes from the Law Library.

E-book volumes added for the following collections: Blackwell Reference Online, Springer e-book annual collection (CIC), Humanities e-books, and individual titles (ordered from various publishers one by one). 17,082 total volumes were added. Includes 17,082 e-books as well as reporting from Law, Music, and Lilly (Spec Coll) libraries.

Reported titles accessible via aggregators. This number excluded in 2007-2008.

More titles are available in this category. The library increased its efforts to make these titles available in 2008-2009.

33,234 withdrawn in 2008-2009 as print material was reviewed for storage to open up space in the Government Documents stacks. 
Question Footnote

Number

INDIANA, cont.

10

24.b

26

28

33

36

37- 38

39

40

41-43

IOWA

1.a

18.a

20

25

27.a

28

IOWA STATE

1.a

1.b.i

3

14

20

28

Archives holdings not reported in 2007-2008, but reported in 2008-2009.

Indiana State Library through INSPIRE provides mainstream databases.

Increased expenditure due to more accurate reporting and 7\% increase in borrowing due to WorldCat Local implementation in December 2008.

Dormitory video libraries reported as service points in 2008-2009. Figure reflects video records in the library catalog.

Circulation from the Main Library (Wells) Storage Facility (ALF) and dormitory media libraries increased significantly in 2008-2009. Reserve charges included. For past several years, ILS did not distinguish between initial charges and reserve charges.

7\% increase in borrowing due to WorldCat Local implementation in December 2008. ILL staff also processed 13,553 local user requests for document delivery.

Includes Law Library data.

Includes 69 fulltime Law School faculty.

Includes 220 Law School graduate students, assumed to be full-time.

Includes Law Library data.

Library branches included: Art, Biological Sciences, Business, Engineering, Geoscience, Mathematical Sciences, Physics, Music, and Psychology.

Data from the Law and Health Science Library are also included in the figures reported.

Volumes held June 30, 2008 revised to 5,014,189 which includes 3,848,262 volumes from the Main Library, 815,369 volumes from the Law Library, and 350,558 volumes from the Health Science Library. Figure includes added e-books for the year. Includes programming staff that support the Libraries but not directly from Libraries' budget.

Includes equipment costs that support the Libraries but not directly from Libraries' budget.

Includes hard/software costs that support the Libraries but not directly from Libraries' budget.

Includes 3 FTE programmers who support the Libraries but not paid directly from Libraries' budget.

Includes branch service points.

Library branches included: Main Library and Veterinary Medical Library, plus three reading rooms (Design, Physical Sciences, and Math) are included in Main Library figures.

Volumes held June 30, 2008, new figure of 2,537,424 equals 2,529,921 (reported in 2007-2008 ARL Statistics) plus 7,503 (number of electronic books held, reported in Q1a of the 2007-2008 ARL Supplementary Statistics, but not included in the "Volumes held June 30, 2008" in the 2007-2008 ARL Statistics.)

48,757 includes 8,061 e-books. (Complete breakdown by product is not available.)

28,369 includes 306 e-books. (Breakdown by product is not available.)

The Film and Video count dropped in 2008-2009 due to the transfer of approximately 44,000 films in the American Archives of the Factual Film (AAFF) collection to the Library of Congress.

Significant increase in Other operating expenditures due to concerted effort to expend gift funds in 2008-2009 on materials acquisitions, furniture, and equipment. Utilities of $\$ 699,279$ and fringe benefits of $\$ 2,435,616$, which were moved into the Library's budget in 2008-2009, are excluded from total.

In 2008-2009, the Interlibrary Loan/Document Delivery process moved to Technical Services, the ILL/DD service desk closed, and point of service was transferred to the Circulation Desk. Also in 2008-2009, Microforms, Maps, and Reserve merged into the newly renovated Media Center. 
Question Footnote

Number

JOHNS HOPKINS

Library branches included: Milton S. Eisenhower Library, Welch Medical Library, Institute of the History of Medicine, Arthur Friedheim Library and J. Peabody Institute Archives, SAIS Mason Library, Washington Centers.

Johns Hopkins facility in Florence, Italy was sold, including all of its library materials. Data from the Medical Library are also included in the figures shown.

1.a

\section{KANSAS}

1.b.i, 3

5.a

5.b

16.c

41-43

KENT STATE

1.a

5

5.a

11

27.c

\section{KENTUCKY}

3

5.a.i

5.b.i

5.b.ii

5.b.iv
Applied Physics Library closed its physical location 10/1/2009. The physical collection was dispersed throughout 2008-2009. 2008-2009 financial data not available from APL.

Volumes held June 30, 2008 revised to 3,730,439.

Library branches included: Regents Center Library and Edwards Campus (Overland Park, KS).

Data from the Law and Health Science Library are also included in the figures reported.

Lower 2008-2009 print book total reflects approval plan reductions. 2008-2009 e-book total is lower than 2007-2008 because

2007-2008 included 13,307 Springer e-books. No e-book collection of comparable size was acquired in 2008-2009.

In 2008-2009 added new databases that increased access to online full-text journals (chiefly IEEE/IET Electronic Library).

Changed count method from 2007-2008.

2007-2008 total reflects more 1-time digital collection purchases and more non-book material purchases than 2008-2009.

Beginning Fall 2008 all graduate students in thesis or dissertation preparation are counted as full-time per federal IPEDS definition.

Library branches included: Main, Architecture, Chemistry/Physics, Fashion, Map, Mathematics \& Computer Sciences, and Music.

Library branches NOT included: Regional campus libraries (NOT on the Kent campus).

Volumes held June 30, 2008 revised to 2,853,956.

Serial titles are not deduplicated.

The numbers reported represent an estimate and are not deduplicated. Serials have been difficult to deduplicate by titles rather than by subscription since serials now come in multiple and differing formats. This estimate in serial titles has resulted in an increased number of serials but a reduction in the expenditures for serials. Thus, our unit price for serials purchased may be unrealistically low.

Count of cartographic materials in past years included only cataloged items. 2008-2009 count includes map library's inventory of all items.

The 2008-2009 figure reflects our use of a 40 hour work week as full-time for students.

Library branches included: Agricultural Information Center, Design Library, Education Library, Engineering Library, Equine Library, Little Fine Arts Library, Kentucky Transportation Center Library, Law Library, Medical Center Library, Science Library, Special Collections Library, and William T. Young Library.

Total includes 13,130 e-books.

Includes Main Library and Medical Library electronic serial titles. We are unable to break out a separate total for the Medical Library.

Includes Law and Medical libraries. We are unable to break out separate totals for the Law and Medical libraries.

Includes Law and Medical libraries freely accessible serial titles. We are unable to break out separate totals for Law and Medical libraries.

We are unable to deduplicate government documents serial titles. 
Question Footnote

Number

LAVAL

All figures are as of 05/31/2009.

Library branches included: We have one library in two buildings: Social and Human Sciences Library (including Law) and the Sciences Library Building (including Medicine). Data from all are included in the 2008-2009 ARL Statistics.

16-26 Expenditures as reported in Canadian dollars: (16a) $\$ 2,554,595 ;$; (16b) $\$ 8,156,018 ;(16 c) \$ 165,485 ;$; (16d) $\$ 362,488$; (16) $\$ 11,238,586$;

(17) \$59,253; (18a) \$4,289,953; (18b) \$7,165,192; (18c) \$26,162; (18) \$11,481,307; (20) \$670,394; (21) \$23,449,540; (22) \$187,598; (23)

$\$ 5,106,870$; (24a) \$0; (24b) \$0; (25) \$0; (26) \$56,238.

19

Total fringe benefits not included are $\$ 3,075,983$.

\section{LOUISIANA STATE}

Data from the Law and Health Science Library are also included in the figures reported. Many changes from 2007-2008 were due to mid-year budget cuts and a hiring freeze.

1.a

Volumes held June 30, 2008 revised to 4,085,637 which includes 3,583,461 volumes from the Main Library, 453,368 volumes from the Law Library, and 48,808 volumes from the Health Science Library.

1.b Increase due to budget changes.

1.b.i, 5.a-5.a.i Increase due to budget changes and/or changes in staffing patterns.

1.b.ii

5.a.ii

5.b

5.b.iii

Decrease due to budget changes and/or changes in staffing patterns.

Decrease due to budget changes and/or changes in staffing patterns. Note: This question was originally marked UA/NA, but was changed so that the operating system (StatsQual) could aggregate sub-totals reported in the Law and Health Sciences surveys.

Receivals from consortial membership(s) were 0 for all libraries in 2008-2009.

Increase due to discontinuation of 4 titles at the Law Library.

13-14, 16-16.a, Decrease due to budget changes and/or changes in staffing patterns.

16.c, 20-22, 25, 27,

27.a, 27.b

27.c

35

Increase due to budget changes and/or changes in staffing patterns.

39

Joined RAPID consortium and provided 9,689 for those institutions and 6,030 via OCLC/ILLiad.

Decrease due to the loss of 4 faculty in the Law Library.

\section{LOUISVILLE}

Library branches included: Music Library, Art Library, Health Sciences Library, and the University Archives.

\section{MCGILL}

All figures are as of 05/31/2009.

Library branches included: Birks Reading Room, Education Library, Islamic Studies Library, Life Sciences Library, Humanities \& Social Sciences Library, Blackader-Lauterman Library of Art \& Architecture, Marvin Duchow Musich Library, Howard Ross Library, Macdonald Campus Library (agricultural \& Environmental Sciences), Osler Library of the History of Medicine, Nahum Gelber Law Library, Schulich Library of Science \& Engineering, Edward Rosenthall Library of Mathematics \& Statistics, Walter Hitschfeld Georgraphic Informatic Centre.

Data from the Health Sciences Library are also included in the figures reported.

Volumes held revised to 4,067,649 which includes 3,380,725 volumes from the Main Library and branch libraries (not including Law and Health Sciences), 191,167 volumes from the Law Library, and 495,757 volumes from the Health Sciences Library. This figure includes 101,619 e-books previously unreported. 
Question Footnote

Number

MCGILL, cont.

3

$16-26$

39

\section{MCMASTER}

1

1.a

3

9,14

11

$16-26$

20

24.a-24.b

MANITOBA
We purchased many new e-book packages as well as new titles for packages previously purchased: Apabi e-books, Blackwell Reference Online, Books 24x7 (additional titles), Cambridge Histories Online, Cambridge University Press e-books, Canadian Electronic Library Publishers Collection for health and public policy, Classical Scores Library, Defining Gender, DLB, Dram, Early Canadiana Online, Evans Early American Imprints, Gale Virtual Reference (additional titles), Knovel (additional titles), LWW Medical Book Collection, LLMC Digital (additional titles), Macmillian cabinet papers, Naxos Music Library, NetLibrary ${ }^{\circledR}$ (additional title), Oxford Digital Reference shelf, Oxford Scholarship Online, Oxford University Press e-books, Past Masters PEP Archive (Psychoanalytic Electronic Publishing), PsycBooks, Safari Tech books (additional titles), SourceOECD, Si Ku Quan Shu Database with 3,461 e-books, Springer 2008 \& 2009 e-books (MyiLibrary collection), Taylor \& Francis e-book package, United States Congressional Serial Set, Wiley e-books, World Bank (additional titles), FoodNetBase, NutritionnetBase

Expenditures as reported in Canadian dollars: (16a) $\$ 4,433,398$; (16b) $\$ 9,648,003$; (16c) $\$ 245,076$; (16d) $\$ 828,320 ;(16) \$ 15,154,797 ;$ (17) \$202,755; (18a) \$5,504,792; (18b) \$6,878,996; (18c) \$468,685; (18) \$12,852,473; (20) \$4,865,278; (21) \$33,075,303; (22) \$743,242; (23) $\$ 8,201,983$; (24a) \$0; (24b) \$0; (25) \$1,164,436; (26) \$49,434.

The figure for the number of full-time instructional faculty was taken from the McGill factbook.

All figures are as of 04/30/2009.

Library branches included: Mills Memorial Library, H.G. Thode Library of Science \& Engineering, Innis (Business) Library, and the Health Sciences Library.

All figures are as of 04/30/2009. After much discussion we have decided to move to a bibliographic count for this question, resulting in a total of 1,869,330 volumes, which includes 368,191 e-books. This will be our base count moving forward. The figure used in previous years was a mixture of physical and bibliographic counts.

Volumes held revised to 1,891,993 which includes 1,747,078 volumes from the Main Library and 144,915 volumes from the Health Sciences Library. The 2007-2008 figure $(2,135,520)$ was a mix of physical and bibliographic counts.

46,416 e-books were purchased in 2008-2009. 3,601 were title by title purchases. 3,585 were short term loans, and 39,230 were purchased through consortial packages.

This figure is now a bibliographic count.

This collection was weeded in 2008-2009. 7,742 items were removed from the collection of satellite images.

Expenditures as reported in Canadian dollars: (16a) \$1,380,743; (16b) \$6,611,308; (16c) \$212,071; (16d) \$1,107,900; (16) \$9,312,022;

(17) \$7,233; (18a) \$4,056,082; (18b) \$3,599,220; (18c) \$635,624; (18) \$8,290,926; (20) \$2,747,842; (21) \$20,358,023; (22) \$189,974; (23) $\$ 4,596,670$; (24a) \$0; (24b) \$0; (25) \$644,529; (26) \$49,591.

This figure includes equipment costs that were expensed as part of the classroom renewal project. Classroom and $\mathrm{AV}$ services are now a unit of the library.

These charges are now included in Acquisitions Support, not operating expenditures.

All figures are as of 03/31/2009.

Library branches included: William R. Newman Library, Architecture Fine Arts Library, Archives \& Special Collections; Elizabeth Dafoe Library; Fr. Harold Drake Library; St. John's College Library; Donald W. Craik Engineering Library; E.K. Williams Law Library; Albert D. Cohen Management Library; Eckhardt-Gramatte Music Library; Sciences and Technology Library; Neil John Maclean Health Sciences Library; Bill Larson Library; Carolyn Sifton-Helen Fuld Library; Concordia Hospital Library; J.W. Crane Memorial Library; Misericordia Hospital Library; Riverview Health Centre Virtual Library; Seven Oaks General Hospital Library; Victoria General Hospital Library. 
Question Footnote

Number

MANITOBA, cont.

1.b.ii Increase due to intensive weeding and collections maintenance in preparation for move of materials to newly constructed storage unit.

5.b.iv It is not possible to provide a separate count for government publication serials received but not purchased for $2008-2009$.

16-26 Expenditures as reported in Canadian dollars: (16a) \$1,972,454; (16b) \$6,577,890; (16c) \$120,997; (16d) \$377,594; (16) \$9,048,935;

(17) $\$ 152,555 ;$; (18a) $\$ 5,627,502 ;$ (18b) \$5,842,452; (18c) \$796,011; (18) \$12,265,965; (20) \$2,138,318; (21) \$23,605,773; (22) \$647,336; (23)

$\$ 4,267,405$; (24a) \$0; (24b) \$0; (25) \$476,910; (26) \$169,439.

16.c This figure represents expenditures on serial back files only. It is not possible to disaggregate expenditures for charts, maps, audiovisual materials, manuscripts, etc.

MARYLAND

2

Library branches included: Art, Architecture, Chemistry, EPSL, McKeldin, Hornbake, PAL, Shady Grove.

University of Maryland Libraries do not track this data.

\section{MASSACHUSETTS}

3

37

MIT

1-1.a

1.b

3

18-18.c

MIAMI

1

1.a

2

2

5.b.iv

6

Library branches included: Integrated Sciences and Engineering Library, Music Listening Lab, and Image Collection library.

56,106 e-books were also purchased at a cost of $\$ 220,090$.

Excludes 6 DNP (Doctor of Nursing Practice), 1 Au. D. (Audiology), and 29 Ed.D. degrees.

Volumes held June 30, 2008 revised to 3,017,034. Figure revised to include 102,982 EEBO e-books.

Volumes added during the year includes the following counts: 40,962 print volumes, 6,904 e-books, 3,642 e-theses, and 2,223 digitized volumes.

Number of monographic volumes purchased includes the following counts: 22,416 print titles and 253 individually purchased e-book titles.

Includes fringe benefits.

All figures are as of 05/31/2009.

Library branches included: Richter Library (central library); Calder Memorial Library (medical library); Law library; and the Architecture, Business, Marine Science, and Music libraries. [Central Library (Richter)].

Includes 550,975 ETDs and e-books, including titles in the following collections: eBrary, NetLibrary, Early English Books Online, Gale's 18th Century Collections Online, Women Writers Project, Wright American Fiction, Making of the Modern World, Making of Modern Law, Literature Online, Sabin, Evans, Shaw-Shoemaker, Gale Virtual Library, Knovel, Safari Tech Books, Thieme, Synthesis, and OVID. [Central Library (Richter)].

Volumes held revised to 3,264,296 which includes 2,598,070 volumes from the Main Library, 448,545 volumes from the Law Library, and 217,681 volumes from the Medical Library. Differs from volume count reported in 2007-2008 by 1,207 volumes due to error in music library bound periodicals count. [Central Library (Richter)]

Includes print, e-book, and microform titles in the ILS database. Does not include non-recon titles, e.g., pre-1976 government documents, special collections, and a small percentage of general collections (volume counts for the latter are included in our volumes held, as we kept an historic physical volume count prior to the use of an ILS). [Central Library (Richter)] Included in central library (Richter) statistics. [Marine Library]

Increase due to the inclusion of subtotals from the Law Library. This survey question was originally marked UA/NA, but it was changed so that sub-totals from the Law survey could be aggregated to the overall serials figures. [Central Library (Richter)]

Only e-serial government document titles are included for the central library. [Central Library (Richter)] 
Question Footnote

Number

MIAMI, cont.

9

10

13

14

18

27.a

27.b

30-31

32.a

\section{MICHIGAN}

1.a

1.b

5

7
Medical library computer files are reported in Q14. [Central Library (Richter)]

Includes linear feet added to University Archives in 2008 and 2009; 2007-2008 figure should have been 17,940, not 17,932 as reported. [Central Library (Richter)]

Includes 6,345 e-music titles. [Central Library (Richter)]

Medical library audiovisual materials (questions 11 through 14) and computer files (Q9) are reported in Q14. [Central Library (Richter)]

Excludes salaries of the University Librarian and directors of the law and medical libraries. [Central Library (Richter)]

Includes library faculty and selected non-faculty professional staff. Medical library reports all non-faculty professional staff in Q27b. [Central Library (Richter)]

Includes both support and selected non-faculty professional staff. Medical library reports all non-faculty professional staff in Q27b. [Central Library (Richter)]

Central, architecture, business, marine science, and music libraries include library research consultations in library instruction sessions. [Central Library (Richter)]

Includes transactions for University archives and architecture library, not reported in 2008. Transactions are based on enumerations in central, business, and medical libraries, and sampling in architecture, law, marine science, and music libraries. [Central Library (Richter)]

Library branches included: University Library, Bentley Historical Library, William L. Clements Library, Kresge Business Administration Library, and the Law Library.

Library branches NOT included: Dearborn and Flint campuses.

Data from the Health Science Library are also included in the figures reported.

Volumes held June 30, 2008 revised to 9,472,388 which includes 8,203,508 volumes from the Main Library, 712,384 volumes from the Law Library, and 556,496 volumes from the Health Science Library. This number does not agree with the "volumes held June 30, 2008" reported in the 2007-2008 ARL statistics because it includes 299,755 digitizations of volumes in the public domain (and hence available full text) that were added to the HathiTrust digital repository in 2008-2009 and which are accessible through our catalog.

Consolidation and deduplication of collections resulted in an increase in volumes withdrawn. This number includes 101,030 physical volumes and as well as 40,933 e-books added during the year.

Owing to the way we keep our records, we cannot break down our serials into these categories while still deduping on ISSN. Catalogued serials with ISSNs have been deduped within and across libraries. The reported figure also includes 22,343 cataloged serials without ISSNs and 7,388 serials which have not been cataloged; neither of these categories has been deduped.

We no longer count microform by physical unit. Our most recent count by that method was 8,445,189 reported in $2006-2007$. This number remains reasonably accurate as we acquire very little material in this form anymore and we do not withdraw any of it.

Vendored scanning for digitization resulted in an increase in miscellaneous library materials expenditures for the year.

MICHIGAN STATE

Library branches included: Biomedical \& Physical Sciences, Business, Dubai, Engineering, Gull Lake, Mathematics, and the Veterinary Medical Center.

Data from the Law Library are also included in the figures reported. 
Number

\section{MICHIGAN STATE, cont.}

\section{MINNESOTA}

Volumes held June 30, 2008 revised to 5,219,524 which includes 5,064,224 volumes from the Main Library and 155,300 volumes from the Law Library. 2007-2008 volumes held adjusted due to 225,491 e-books packages added as a result of one time downloads.

Increase primarily due to the increase of titles in 4 groups: HeinOnline packages - in 2007-2008 we did not have any of these titles from the consortium in these packages and we have 1,950 in 2008-2009; Academic OneFile - Increase in titles in this package of approximately 3,278; General Business File ASAP - Increase in titles in this package of approximately 1,200; and Wilson Select Plus - Increase in titles in this package of approximately 684 titles.

Increase primarily due to the increase of titles in 2 groups: Directory of Open Access Journals package: Increase of approximately 1390 titles from last fiscal year; and Freely Access packages: Increase of approximately 1,805 titles from 20072008.

Decrease due to a significant serials project which resulted in cancelations and reconciliation of holdings.

Expenditures for one-time electronic resources rose as we converted purchase of a major publisher's books to online format. Expenditures for ongoing electronic materials rose as we continued to convert print + online subscriptions to online-only. Includes HATHI TRUST for the first time this year.

We purchased computers for public workstations in 2007-2008. No large purchase reported for 2008-2009.

The 2007-2008 total was incorrect. Actual 2007-2008 full time enrollment was 40,435

The 2007-2008 total was incorrect. Actual 2007-2008 part time students was 5,610.

The 2007-2008 total was incorrect. Actual 2007-2008 full time graduate students was 7,347.

The 2007-2008 total was incorrect. Actual 2007-2008 part time graduate students was 2,626.

Library branches included: All Twin Cities campus libraries.

Library branches NOT included: University of Minnesota coordinate campuses.

Data from the Law and Health Science Libraries (Bio-Medical, Veterinary Medicine) are included in the figures reported. Volumes held June 30, 2008 revised to 6,916,178 which includes 5,620,539 volumes from the Main Library, 747,569 volumes from the Law Library, and 548,070 volumes from the Health Science Library. Adjusted for 12,024 previously counted volumes for which new online item records were created.

Increase due to unusually large number of duplicated withdrawn for digitizing without return, as part of our participation in the Google duplicates project.

Best estimate available for all University of Minnesota libraries including coordinate campuses.

Count of actual volumes purchased is not available. This figure counts orders placed for monographs and is consistent with previous years' reports. 
Question Footnote

Number

MINNESOTA, cont.

Unique title count is done at the institutional level for $\mathrm{U}$ of $\mathrm{M}$ Libraries, including Health Sciences and Law. Unique titles counts are not calculated separately for branches. Count uses same methodology as previous years:

A. Purchased

1. Identify all purchased electronic titles using query
a. W85=http and WFT=SE not (W85=gpo or W85=gov or WGP=F or WGP=I or WGP=S) and WSL=?INTE not WSL $=$ AINTE (i.e. purchased)
b. Removes gov docs
c. Eser_pur
d. $\quad 36,160$ in 2007
e. $\quad 44,702$ in 2008
f. $\quad 78,239$ in 2009

2. Run bib IDs through p_print_03 to get ISSNs from 022
a. Not all the records retrieved in 1a have an 022 field
b. Not unique
c. $\quad 34,108$ in 2007
d. $\quad 40,760$ in 2008
e. $\quad 48,467$ in 2009

3. Run ARC query to get all Bib IDs and ISSNs associated with a Purchase Method order record
a. ser_pur_ord
b. We first made the mistake of limiting to AC and AP order statuses
c. Later we realized that we need to get all our holdings, not just active subscriptions so we removed the status filter
d. Not unique
e. 21,775 in 2007
f. $\quad 30,992$ in 2008
g. $\quad 32,959$ in 2009

4. Compare the ISSNs from \#2 with the ISSNs from \#3 using Erik's script and dedupe into two buckets:

a. Purchased electronic titles

i. Includes any overlap between electronic and other formats

ii. $\quad 29,486$ in 2007

iii. $\quad 35,513$ in 2008

iv. $\quad 47,632$ in 2009

b. Purchased titles in print/other format only (i.e., not held electronically)

i. Not counting active subscriptions - counting held titles

ii. $\quad 20,752$ in 2007

iii. $\quad 20,812$ in 2008

iv. $\quad 22,865$ in 2009

c. Overlap (i.e., in both files)
i. $\quad 10,227$ in 2007
ii. $\quad 10,170$ in 2008
iii. $\quad 10,094$ in 2009 
Question Footnote

Number

MINNESOTA, cont.

B. Not Purchased

1. Identify all non-purchased electronic titles using query
a. W85=http and WFT=SE MINUS W85=http and WFT=SE not (W85=gpo or W85=gov or WGP=F or WGP=I or $\mathrm{WGP}=\mathrm{S}$ ) and WSL=?INTE not WSL=AINTE (ie not purchased)
b. i.e., start with any serial record with a link and then take out those that are not gov docs
c. Eser_not_pur
d. 16,293 in 2007
e. $\quad 17,867$ in 2008
f. 20,368 in 2009

2. Run bib IDs through p_print_03 to get ISSNs from 022

a. Most of these records do not have an 022 field

b. Since we don't expect to have order records for these titles, we plan to add the full number of records without ISSNs back into the total at the end.

i. In 2006-2007, this number was 13,000

ii. In $2007-2008$, this number was 13,571

iii. In 2008-2009, this number was 14,913

c. Not unique
i. $\quad 3610$ in 2007
ii. $\quad 4296$ in 2008
iii. $\quad 5455$ in 2009

3. Run ARC query to get all Bib IDs and ISSNs associated with a Non-Purchase Method order record
a. Ser_notpur_ord
b. Not unique
c. $\quad 8099$ in 2007
d. 11,976 in 2008
e. 11,888 in 2009

4. Compare the ISSNs from \#6 with the ISSNs from \#7 using Erik's script and dedupe into two buckets:
a. Non-purchased electronic titles
i. Includes any overlap between electronic and other formats
ii. $\quad 3467$ in 2007
iii. $\quad 4127$ in 2008
iv. $\quad 5350$ in 2009
b. Non-purchased titles in print/other format only (i.e., not held electronically)
i. $\quad 10,741$ in 2007
ii. $\quad 11,052$ in 2008
iii. $\quad 10,900$ in 2009
c. Overlap (i.e., in both files)
i. $\quad 804$ in 2007
ii. $\quad 924$ in 2008
iii. $\quad 988$ in 2009 
Question Footnote

Number

MINNESOTA, cont.

5.b

12-14

16.c

16.d

42

MISSOURI

5

\section{MONTREAL}

1.a

$16-26$

24.b

\section{NEBRASKA}

1.a

1.b, 3, 16.a

1.b.ii

5.a.ii
5. Add in the non-purchased titles without ISSNs to get total of electronic titles
a. $\quad{ }^{* *}$ See $\# 2 b^{* *}$
b. $\quad 3467+13,000=16,467$ in 2007
c. $\quad 4127+13,571=17,698$ in 2008
d. $5350+14,913=20,263$ in 2009

Breakdown for consortial, freely accessible, and documents per the survey is not available. Total number of unique nonpurchased titles is 20,263, which includes 10,900 print titles.

Breakdown by format not available. Total AV items is 905,112.

Decrease due to fewer purchases of electronic serial back runs.

Figure reflects expenses from collections budget for ILL and from shared digital repository.

Includes professional.

Library branches included: Archives, Math, Engineering, Geology, Journalism, Vet Med and Health Sciences.

Data from the Law Library are also included in the figures reported.

Editor's Note: All serials figures for the Ellis Library estimated based on 2007-2008 data with the exception of the consortial figures (5.b.i), which were increased from 6,588 titles in 2007-2008 to 14,767 titles in 2008-2009.

All figures are as of 05/31/2009 [Bibliothèques UdeM, École Polytechnique de Montréal].

Data from the Law (Droit), École Polytechnique de Montréal, HEC Montréal and Santé (Health Sciences) Library are included in the figures reported [Bibliothèques UdeM].

Library branches NOT included: Bibliothèques UdeM, Droit (Law), Santé (Health sciences), and HEC Montréal. [École Polytechnique de Montréal].

Volumes held revised to 3,175,855 which includes 2,010,825 volumes from the Bibliothèques UdeM, 281,068 volumes from the École Polytechnique de Montréal Library, 363,103 volumes from the HEC Montréal Library, 183,218 volumes from the Law (Droit) Library, and 337,641 volumes from the Santé (Health Sciences) Library. This error explains the difference of 7,198. [Bibliothèques UdeM]

Expenditures as reported in Canadian dollars: (16a) \$3,082,194; (16b) \$10,999,352; (16c) \$180,688; (16d) \$26,867; (16) \$14,289,101;

(17) $\$ 163,220 ;$; (18a) \$8,060,914; (18b) \$11,030,824; (18c) \$175,247; (18) \$19,266,985; (20) \$1,062,610; (21) \$34,781,916; (22) \$245,484; (23)

$\$ 8,192,202 ;$ (24a) \$81,847; (24b) \$0; (25) \$481,069; (26) \$236,614.

2007-2008 figure should have been 0. [Bibliothèques UdeM]

Library branches included: Don Love Memorial Library, Mathematics Library, Music Library, Architecture Library, Geology Library, Engineering Library, and the C. Y. Thompson Library.

Data from the Law Library are also included in the figures reported.

Volumes held June 30, 2008 revised to 3,204,058 which includes 2,962,248 volumes from the Main Library and 241,810 volumes from the Law Library.

The increase is largely due to the fact that the values for 2007-2008 were uncharacteristically low because the Libraries switched approval plan vendors in 2007-2008.

In the 2007-2008, UNL Libraries launched a significant weeding project. The 2008-2009 figure is actually a typical year of withdrawals.

UNL Libraries Serials Review process included decreasing the amount of print serials in favor of electronic serials. 
Question Footnote

Number

NEBRASKA, cont.

5.b.iii

14

16.d

17

22

23

24.a-24.b

26

31

32

33

42

NEW MEXICO

1.a

1.b.ii

3

5.a.i

5.a.ii

5.b.i

5.b.ii

5.b.iii

9

13

14

16.b

16.d

17

The decrease is due to UNL Libraries' preference for electronic materials.

UNL Libraries increased resources towards purchasing items frequently requested via ILL. The bulk of these materials are audiovisual materials; which are highly in demand.

Library material expenditures have been largely devoted to electronic serials. We expect this trend to continue.

The move towards electronic materials has diminished the need to bind materials. In addition, the use of shrink wrap has reduced the need to bind materials as well.

UNL Libraries is intentionally refraining from purchasing these types of resources as part of the Serials Review Process, which explains the decrease from the 2007-2008 figure.

UNL Libraries renewed a number of large databases licenses this year. These big deals typically occur every three years.

The Libraries decreased the amounts that were paid into the OCLC accounts.

This decrease is largely due to the decrease in shipping costs as well as UNL Libraries switching shipping companies.

This decrease may be due to the fact that many liaison librarians have switched subject areas. This decrease appears to be a trend for UNL Libraries.

In May of 2009, UNL Libraries moved the QuestionPoint (collaborative virtual reference service) box to the front page of the newly designed Library website.

In house use of materials has increased significantly.

Due to the poor state of the economy, graduate student numbers are up.

Library branches included: Centennial Science \& Engineering Library, Fine Arts \& Design Library, Parish Memorial Library, Zimmerman Library, Bainbridge Visual Resources Library and the Tireman Library.

Library branches NOT included: Gallup Campus Branch Library, Los Alamos Campus Branch Library, Taos Campus Branch Library, and the Valencia Campus Branch Library.

Data from the Law and Health Sciences Library are also included in the figures reported. The Native American Studies Library is no longer a staffed library; rather, it is now a reading room. Collections from this library are no longer included in this report.

Volumes held June 30, 2008 revised to 3,013,580 which includes 2,616,973 volumes from the Main Library, 242,357 volumes from the Law Library, and 154,250 volumes from the Health Sciences Library. Previous total did not include e-Books.

Conducted a routine maintenance of our print collection.

Total includes e-Books.

Continued to add titles in early 08/09 using fire insurance funds.

Implemented a project to purchase serials in electronic format whenever possible.

Gale agreement with New Mexico State Library provided more free resources.

More serials are freely available this year. Additionally, more accurate tracking is possible using new internal systems.

Gifts acceptance policy has been narrowed resulting in a decrease.

Bainbridge Visual Resources Library conducted a major initiative to increase their store of digital image files.

Increase due to large collection gift.

Increase due to large purchase of video store collection.

Replacement of destroyed items (due to 2006 fire) is now concluded. Total expenditures have now returned to normal levels.

Added several new memberships and expenditures for some utilities were made off-cycle.

Post-fire increase due to volumes needing binding after their return from a disaster recovery vendor for cleaning. 
Question Footnote

Number

NEW MEXICO, cont.

20

22

24.a

25

26

27.c

29

35

36

NEW YORK

1.a

5, 5.a.i

$5,5 . b$

16.a

27.a

NORTH CAROLINA

All figures are as of $08 / 31 / 2009$.

Library branches included: Bobst Library, Institute of Fine Arts (IFA) Library, Real Estate Institute (REI) Library, Courant Institute of Mathematical Sciences (CIMS) Library, Institute for the Study of the Ancient World (ISAW) Library, Law School Library, Medical School, and Dental School Libraries.

Volumes held revised to 5,079,515 which includes 4,073,311 volumes from the Division of Libraries, 782,290 volumes from the Law Library, and 223,914 volumes from the Health Science Libraries.

Increase primarily reflects larger number of titles under control via SFX that were previously held but not included in the database. There is also some impact from the addition of full access to all titles in IEEE Engineering Society library in Fiscal Year 2009 added in response to the new relationship between NYU and Brooklyn Polytechnic.

Increase due in part to open access growth; also, an increased number of titles under control via SFX were previously held but not included in the database.

Monograph expenditure figures reflect all one-time purchases, including large electronic and other acquisitions, which vary significantly from year to year. Actual expenditures for monographs have increased only marginally. Increase in archival staff headcount due to increase in new short-term archival projects in 2008-2009.

Library branches included: Institute of Marine Sciences Library.

Data from the Law and Health Science Library are also included in the figures reported.

1

Includes government documents.

$1 . a$

Volumes held June 30, 2008 revised to 6,616,164 which includes 5,916,048 volumes from the Main Library, 361,087 volumes from the Law Library, and 339,029 volumes from the Health Science Library. Figure includes 89,340 e-books that represent a large load of records for the Serial Set (Lexis Nexis), but the purchase was made before 2008-2009.

5 Serial total includes 14,874 titles acquired in previous years but not counted until 2008-2009 and 13,587 titles lacking ISSNs that could not be accounted for using prior methodology.

16.d Includes payments for CRL memberships.

21

Includes $\$ 6,317,893$ in grants, trust, and other special funds (without benefits); excludes $\$ 330,125$ in expenditures for the Triangle Research Library Network from funds contributed by Duke, North Carolina State, and North Carolina Central Universities. Also includes 11.7 FTE professional and support staff on special grant funds and 6.4 FTE on special projects; excludes 3.25 FTE paid from funds contributed by Duke, North Carolina State, and North Carolina Central Universities for support of the Triangle Research Libraries Network. 
Question Footnote

Number

\section{NORTH CAROLINA, cont.}

37

\section{NORTH CAROLINA STATE}

All figures are as of 06/30/2008.

Library branches included: Design, Natural Resources, Textiles, Veterinary Medical, African-American Cultural Center Reading Room, and College of Education Media Center.

1

1.a

1.b

3

16.a

$16 . b$

$16 . c$

16.d

17,25

$18,18 . b$

20,22

23

24.a, 26

33- 34

35

36

\section{NORTHWESTERN}

All figures are as of 08/31/2009.

Library branches included: Science and Engineering Library, Math and Geology Libraries, and the Law, Health Sciences and Schaffner Libraries on the Chicago campus.

1

1.a

5.b Includes 407,796 e-books.

Volumes held June 30, 2008 revised to 4,081,278. Figure reflects the addition of 100,000 volumes to the 2007-2008 figure to account for the China Conference Proceedings (Eastview).

Fewer volumes added due to budget reversion and spending freeze.

Fewer volumes purchased due to budget reversion and spending freeze. Includes some monographic standing order items paid from serials funds.

Decline in expenditures due to budget reversion and spending freeze. Some monographic titles are paid from serials funds included in $16 \mathrm{~b}$.

Some monographic titles are paid from serials funds.

Decline in expenditures due to budget reversion and spending freeze.

Includes Bibliographic Utilities (\$217,274), Document Delivery $(\$ 203,201)$, and Association Memberships $(\$ 238,247)$.

Decline in expenditures due to budget reversion and spending freeze.

Increase is the result of "banding" reclassification of support staff positions.

Decline in expenditures due to budget reversion and spending freeze.

Increase due to addition of resources and conversion to electronic subscriptions.

Increase reflects reliance on document delivery and interlibrary loan to support content lost due to budget reversion and spending freeze.

Part of increase may reflect efforts to collect textbooks used in courses or to improve effectiveness of OPAC interface.

Increase probably reflects effects of reductions in collections budgets.

Increase may reflect ease of making requests, efficiency of process, or effects of reductions in collections budgets.

Includes government documents.

Volumes held June 30, 2008 revised to 4,846,829 which includes 3,969,225 volumes from the Main Library, 588,177 volumes from the Law Library, and 289,427 volumes from the Health Sciences Library.

Excludes Law and Health Sciences.

Excludes Health Sciences 
Library branches included: Hesburgh Library (Main) includes: Architecture Library, Art Image Library, Business Information Center, Chemistry/Physics Library, Engineering Library, Kellogg/Kroc Information Center, and Mathematics Library. In addition the Kresge Law Library and University Archives have been reported. [Main Library]. The statistic for the number of monographs purchased is understated due to the number of items purchased as part of a collection. When a collection is purchased as one order, it is counted as one volume regardless of the number of items included in the collection. This understates the statistic for volumes purchased for year in which large collections are purchased. However, when these collections are cataloged the individual volumes are added to volumes held in Q1. [Main Library] The subtotals of this question have been changed to UA/NA so that the operating system could aggregate the Law library survey. The serials 45,321 were originally reported as: (5a) 25,083 with subtotals of (5ai) 21,008; (5aii) 4,075 and (5b) 20,238 with subtotals of (5bi) 4,765; (5bii) 11,439; (5biii) 384; (5biv) 3,650. [Main Library] Current serial received, not purchased includes titles that are received from other institutions in exchange for titles that we have purchased. These were previously included in the statistic for purchased serials, but because no dollars are used to obtain these titles directly, the titles obtained in this manner have been included in the statistic for titles not purchased in the current year. [Main Library] Manuscripts/Archives (linear ft.) have been reported for the University Archives. This figure is unavailable for the Main Libraries. Law library reported 0. [Main Library] This figure represents our entire holdings. It consists primarily of archival records and manuscripts but it also includes substantial quantities of printed, microfilm, artifact, audio, visual, film, graphic, and digital materials. Our holdings are organized according to the archival principle of provenance. Our controls over our records are therefore primarily oriented towards the creator of the records and only secondarily on the particular types of materials. [University Archives] Graphic materials housed in the Art Image Library consist of two components; the analog and digital collections. The analog slide collection holds approximately 210,000 to 230,000 35mm slides available to faculty and students for research, teaching, and presentation purposes. The digital collection has over 31,000 digital images. However, due to copyright restrictions these low-resolution images are only available to Art, Art History and Design faculty and are not included in the total graphic materials reported. [Main Library]

Additional audio materials were added from a new fund that was designated by the University for the purchase of sacred music collections in 2008-2009. [Main Library]

Miscellaneous materials expenditures consist primarily of memberships for the purpose of obtaining/accessing publications and literature searching. [Main Library]

The total salaries and wages figure for the University Archives does not include \$152,106 in fringe benefits. It would be very time consuming to apportion the fringe benefits to the three salary classifications given, though the amount can be approximated on a pro rata basis, with only a minimal amount (about $7 \%$ of salary) allocated to student employees and the remainder split between the remaining two categories. [University Archives] Professional staff expenditures have increased by $12 \%$ due to the inclusion of non-faculty professional staff in the amounts reported on line 18.a. (Total Salaries and Wages for Professional Staff) rather than 18.b. (Total Salaries and Wages for Support Staff). Both the FTE and expenditures reported related to professional staff were reported in the support staff lines in 20072008. Had the expenditures for these 7 FTE's been included in the professional staff line in the 2007-2008 fiscal year, the difference would have resulted in an increase of only 4.5\%. [Main Library]

18.a-18.c Fringe benefits not included in 2008-2009 figures. [University Archives] 
Question Footnote

Number

NOTRE DAME, cont.

18.c

OHIO
Expenditures for student assistants increased in 2008-2009 due to an increase in the number of students (8 FTE's for the Main Library) and an increase in student pay rates. [Main Library]

For the Main Library, the increase in other library operating expenditures for 2008-2009 was a result of increases in several categories. The most notable in order of greatest dollar significance were: information technology $=33.8 \%$; supplies $=23.5 \%$; renovation $=216.1 \%$; resource delivery $=41.6 \%$; and travel $/$ professional development $=10.7 \%$. [Main Library]

The University Archives spent less in this category in this year than last. [University Archives]

The total library expenditures figure does not include $\$ 152,106$ in fringe benefits. It would be very time consuming to apportion the fringe benefits to the three salary classifications given, though the amount can be approximated on a pro rata basis, with only a minimal amount (about $7 \%$ of salary) allocated to student employees and the remainder split between the remaining two categories. [University Archives]

Expenditures for one-time electronic resource purchases were less than half of the amount reported in 2007-2008 due to the reduction in spending for bibliographic utilities, networks and consortia from internal library sources (a subset of this statistic) and other planned reductions. See the footnote for line 24.a. [Main Library]

The Main Library realized a 35.5\% savings for expenditures for bibliographic utilities, networks, and consortia from internal library sources due to the cessation of Resource Library Group (RLG) services and a reduction in payments to OCLC during the year. [Main Library]

Computer equipment and peripherals expenditures were greater in 2008-2009 by over \$190,000 compared to 2007-2008 due to the cyclical nature of purchasing replacement equipment. In addition, other computer related expenditures including software and software application fees increased by approximately $\$ 65,000$. [Main Library]

Document delivery/interlibrary loan expenditures have increased by over $40 \%$ due in large part to the increased use of copyrighted material (and associated fees) for electronic reserves. [Main Library]

The FTE for professional staff has increased by $17 \%$ due to the inclusion of non-faculty professional staff in the FTE for line 27.a. rather than the support staff line. Had these 7 FTE's been included in line 27.a. rather than 27.b. support staff in 200708, the difference in FTE's reported would have been an increase of only $8 \%$. This includes an increase of one FTE for an additional librarian in residence. [Main Library]

The FTE for student assistants increased by nearly $29 \%$ due to an increase in student employment for departmental projects in 2008-2009. [Main Library]

The total hours worked by student employees equals less than half of one FTE. [University Archives]

The University Archives provided more tours this year than last. [University Archives]

The change in this statistic for the University Archives was due to improved collection of statistics requiring no sampling, and an increase in reference questions in 2008-2009. [University Archives]

This statistic for the University Archives is based about $80 \%$ on count and about $20 \%$ on a reasonably accurate estimate. [University Archives]

Lending requests filled dropped by $15.8 \%$ due in part to the decrease in requests form materials received (fell by $2.5 \%$ ) and a large drop in OCLC articles filled (from $51.8 \%$ to $41 \%$ filled of requests received). The decrease fill rates are due in part to: a massive location shift of materials during the year which made it difficult to locate materials, a personnel change in the department, and an increase in materials requested that were charged out to our own users $(8,993$ items, almost $24 \%$ of all OCLC requests). [Main Library]

Library branches included: Includes Athens (main), Chillicothe, Lancaster, Southern, Eastern, Zanesville libraries as well as the College of Osteopathic Medicine Learning Resource Center. 


\section{OHIO, cont.}

1

1.a

1.b.i

1.b.ii

5.a

16.a

16.d

18.a

26

35

\section{OHIO STATE}

1

1.b.ii

9

13

14

18.a

32

37
Includes 648,808 e-books. E-book collections include LexisNexis U.S. Serial Set (circa 300,000 titles), Eighteenth Century Collections Online (ca. 136000 titles), U.S. government monographs available online (ca. 90,000 titles), Early American Imprints (ca. 37,000 titles), NetLibrary (ca. 15,000 titles), OhioLINK Electronic Book Center (ca. 15,000 titles), Safari Books Online (ca. 7,500 titles), eBrary (ca. 7,000 titles), Alexander Street Press (ca. 3,500 titles), Learning Express Library (ca. 500 titles), and various individually cataloged e-books.

Volumes held June 30, 2008 revised to 2,953,370. Figure includes 60,132 e-books added to the collection in 2008-2009 as the result of a one-time download (LexisNexis U.S. Serial Set Collection).

Includes approximately 14,000 new e-books added to the catalog (in addition to the 60,132 reported in question 1 ). High rate of electronic book additions due to stepped-up cataloging efforts in the OhioLINK consortium.

Includes 20,000+ volumes withdrawn by Lancaster Campus Library, 14,000+ Dewey classified volumes, and thousands of print serial volumes withdrawn due to duplication in JSTOR, the OhioLINK Electronic Journal Center, and other online sources.

Increase likely reflects increased purchasing of aggregated packages and higher number of titles in bundled/aggregated packages that are now directly accessible to users.

Decrease due to serials inflation, and to shift in some subject areas from purchasing print monographs to subscribing to e-book subject collections.

Miscellaneous expenditures include the following: OhioNET $(\$ 2,100)$, Consortial memberships (\$47,670), Document delivery $(\$ 10,387)$, and OCLC/YBP technical services $(\$ 121,529)$.

Increase reflects university's strategic plan commitment to increasing librarians' salaries.

Document Delivery / Interlibrary Loan expenditures include the following: Borrowing fees (\$9,294), telephone (fax)/ postage/freight $(\$ 20,556)$, OCLC technical service $(\$ 20,684)$, and document delivery $(\$ 10,387)$.

Increase due to shift to a higher lending category in OhioLINK's patron-initiated borrowing system (i.e., an attempt is made to achieve some parity between borrowing and lending levels for each member institution).

Library branches included: Ackerman; Architecture; Biological Sciences/Pharmacy; Business; Cartoon Research; Children's Hospital; Health Sciences; Fine Arts; Food, Agriculture and Environmental Sciences; Law; Music/Dance; Science and Engineering; Sullivant; Theatre Research Institute; University Archives; Veterinary Medicine.

Library branches NOT included: Agricultural Technical Institute; Ohio Agricultural Research and Development Center; Regional Campus Libraries at Lima, Mansfield, Marion, and Newark.

Includes 351,162 e-books such as: Humanities Ebooks (2,252), Chadwick Healey (1,422), eBrary (8,010), English Short Title Catalog (136,425), Evans (37,369), US Serial Set (4,541), NetLibrary (15,227), Safari (9,685), Shaw-Shoemaker (38,092), SpringerLINK (5,070), and locally digitized brittle books $(4,564)$. WWW Theses $(14,673)$ are included in this count for the first time.

Increase is due to closing of the Business Library and planned reduction/deduping of Thompson Stacks collection prior to re-opening in 2009.

Increase due to inclusion of circulation CD-ROMs, which were excluded from the 2007-2008 total in error.

Audio, film, video are combined for Main; cannot separate. Audio/video reported here include 4,045 web audio items and 1,984 web video items with records in the library catalog, in addition to 76,574 physical audio and video items.

Audio, film, video are combined for Main; cannot separate.

Includes faculty and administrative/professional staff salaries.

Reporting actual rather than sampled data for the first time.

Data on Ph.D. degrees awarded during this period is not yet available from the university. 
Question Footnote

Number

OHIO STATE, cont.

39

42-43

OKLAHOMA

1.b-1.b.i

1.b.ii

3

5

5.a.ii

8

16.d

20,25

30

33-34

35

43

\section{OKLAHOMA STATE}

Library branches included: Oklahoma City, Okmulgee, Tulsa.

Data from the Health Science Library are also included in the figures reported.

1.a

3

5.b

12

17

18-18.c

40

\section{OREGON}

1

2

5.a.i, 5.b.ii

16.c

Includes professional students.

Library branches included: Schusterman. [Main Library].

Figure reflects a decrease in items purchased. [Main Library]

Figure reflects an increase in deselection. [Main Library]

Figure reflects an increase in acquisition of e-books. [Main Library]

Figure reflects a decrease in acquisition of print items. [Main Library]

Figure reflects an increase in electronic resources. [Main Library]

Figure reflects increased ILL processing and postage costs. [Main Library]

Library management system was upgraded. [Main Library]

Figure reflects increased library presentations. [Main Library]

Figure reflects new data collection methodology. [Main Library]

Figure reflects increased use as lending library. [Main Library]

Figure reflects an increase in enrollment. [Main Library] from the Health Science Library. full-text tiles in statewide funded databases.

Includes fringe benefits. which these students are classified. Library, Marine Biology Library, Portland Library and Learning Commons.

Data from the Law Library are also included in the figures reported.

Includes 135,360 e-books.

Figure includes 1,665,586 print titles and 135,360 e-books.

Other library materials costs are included in monographs or serials.
Excludes clinical, research, and auxiliary faculty.

Data from the Law and Health Science Library are also included in the figures reported.

Serial statistics are reported as deduped for all libraries following ARL best practices. [Main Library, Schusterman]

Volumes held June 30, 2008 revised to 2,867,866 which includes 2,808,425 volumes from the Main Library and 59,441 volumes

Based on instructions from ARL we included 37,760 e-books purchased in 2008-2009.

This increase is a combination of an increase in the number of open access journals that are cataloged and the addition of

Decrease due to extensive weeding project and decision to count sets of materials rather than individual items.

This decrease is due to the fact that we are no longer binding theses or dissertations. Additionally, the decrease in the purchase of print serials and fewer print government documents means less binding.

This figure does not include students working on their doctorate of veterinary medicine degree who are served by our campus libraries. The IPEDS categories requested in the survey do not include first professional students, the category in

Library branches included: Knight Library (main), Science Library, Mathematics Library, Architecture and Allied Arts

12,779 electronic titles reported as purchased last year are actually freely available and reported correctly this year. 


\begin{tabular}{ll}
$\begin{array}{l}\text { Question } \\
\text { Number }\end{array}$ & Footnote \\
\hline $\begin{array}{l}\text { OREGON , cont. } \\
16 . d\end{array}$ & This includes indexes/abstracts, document delivery, ILL, and bibliographic utilities. \\
22 & Includes the last of a multi-year payment for Eighteenth Century Collections Online. \\
PENNSYLVANIA & \\
& $\begin{array}{l}\text { Data from the Law and Health Science Library are included in the figures reported. } \\
\text { 1.a }\end{array}$ \\
& $\begin{array}{l}\text { Volumes held June 30, 2008 revised to 6,096,588 which includes 5,256,683 volumes from the Main Library, 636,318 volumes } \\
\text { from the Law Library, and 203,587 volumes from the Health Science Library. }\end{array}$
\end{tabular}

\section{PENNSYLVANIA STATE}

Library branches included: All University Park Libraries, as well as the Campus Libraries. Campus Library locations are as follows: Abington, Altoona, Behrend, Berks, Beaver, Capital, Brandywine, DuBois, Fayette, Great Valley, Hazelton, Lehigh Valley, Mont Alto, McKeesport, New Kensington, Schuylkill, Shenango Valley, Wilkes-Barre, Worthington-Scranton, and York.

Data from the Law and Medical Library are also included in the figures reported.
1.b.i The decrease in hard copy is the result of the increase in digital books which are not part of the volume count.
5.b.ii 22,000 reported as freely accessible via SFX. Figure was not available last reporting cycle.
$7 \quad$ Decrease due to improved reporting procedures after revisiting our process in collecting/reporting Library Collection units. 2007-2008 microform count was overstated.
14 Decrease due to improved reporting procedures after revisiting our process in collecting/reporting Library Collection units. 2007-2008 film and video unit count was overstated.

16.c, $22 \quad$ Increase due to difference in year-end close-out strategy.

\section{PITTSBURGH}

1.a

1.b.i

1.b.ii

5

5.a

16.d, 21

27.c

30

39

40

\section{PRINCETON}

1.a

3
Library branches included: All University Library System libraries, Health Sciences Library System, and Law Library of the University of Pittsburgh.

Volumes held June 30, 2008 revised to 5,713,763. 2007-2008 figure was incorrect.

Increase due to the addition of a large number of electronic books this fiscal year.

Health Science Library withdrew a large amount from their collection in 2008-2009, which explains this decrease.

There was an increase in electronic serials purchased this year.

There has been an increase in electronic serials this fiscal year.

Expenditures had to be moved around this year due to the economy and budgetary cuts.

Due to budgetary cuts we had to cut back on student hours used.

This fiscal year we have seen that there were fewer instruction sessions provided; however, there was an increase in the number of participants at each session.

2007-2008 figure included all faculty. 2008-2009 figure only reflected full time instructional faculty.

For questions 40 through 43 the 2007-2008 figures were for the wrong year. Those figures should have been the following: question 40 - 28,713; question 41 - 5,185; question 42 - 6,827; and question 43 - 2,985.

Library branches included: Main library plus 9 additional branches.

2007-2008 count is 6,778,675 and "\% Changed" column should reflect a percent change of $2.3 \%$. Incorrect figure appears in the summary data.

Includes 19,388 e-books. 


\section{PRINCETON, cont.}

5.b.iv

23

PURDUE

\section{QUEEN'S}

Increase due to more Voyager records for Govt. Docs.

Includes e-journals.

Library branches included: The library system on the West Lafayette campus, consisting of 11 subject-oriented libraries, an undergraduate library, and a special collections research center.

Includes cataloged government documents. Includes e-books from the following vendors/sources: ASM handbooks (26), Cognet (490), Credo Reference (426), EEBO (125,000), ECCO (150,000), EngNetBase (105), Gale Virtual Reference (96), ACLS Humanities Ebooks (2,200), Knovel Online ( 1,600), Springer (na), ASAE (4), WorldBank ELibrary (4,500), ASABE (14), and others.

Volumes held June 30, 2008 revised to 2,509,156. Decrease in volumes held is largely due to decrease in gross volumes added. Represents number of titles rather than volumes. Does not include e-books or materials purchased on sponsored research programs. Decrease from previous year reflects decrease in titles purchased and increased accuracy in reporting. Excludes numbered monograph series. Decrease is a result of more accurate reporting, reflecting enhanced clarity in instructions for best practices for deduplicating serial titles.

Total of 13,498 for Q5 cannot be disaggregated for 5bi, 5bii, 5biii, and 5biv. Includes cataloged depository, exchange, and gift serials, and e-journals received through statewide consortium.

Decrease due to the withdrawal of all audio cassettes and laser discs during the year.

Figure reflects major deselection project of videocassettes in 2008-2009.

Figure reflects increase in costs associated with serials.

Decrease reflects more accurate tracking of resources.

Reflects increased expenditures for bibliographic utilities.

Decrease reflects mid-year decision to bind only print-only titles.

Includes fringe benefits.

Decrease is due to reduction in budget.

Includes large one-time purchases through CIC consortium.

Increase reflects increasing costs of licensed electronic resources.

Decrease reflects decision to skip a round of workstation refresh in 2008-2009 and to address server refresh through virtualization and consolidation rather than new equipment.

Increase largely reflects change in schedule of payments to ILL services provider.

Decrease reflects vacant lines that have been closed due to budget reductions and realignment of resources.

Excludes use of online, interactive tutorial "CORE."

Discrepancy when compared to 2007-2008 data reflects return to pre-2007-2008 practice for collecting participant data.

Includes 3,560 reference transactions via digital reference service.

All figures are as of 04/30/2009.

Library branches included: Humanities and Socials Sciences (Stauffer), Engineering and Science (Douglas), Special Collections and Music (Jordan), and Education.

Data from the Law and Health Science Library are also included in the figures reported. Figures for questions 37, 38 and 4043 are as of November 2008. Figure for question 39 is as of October 2008. 


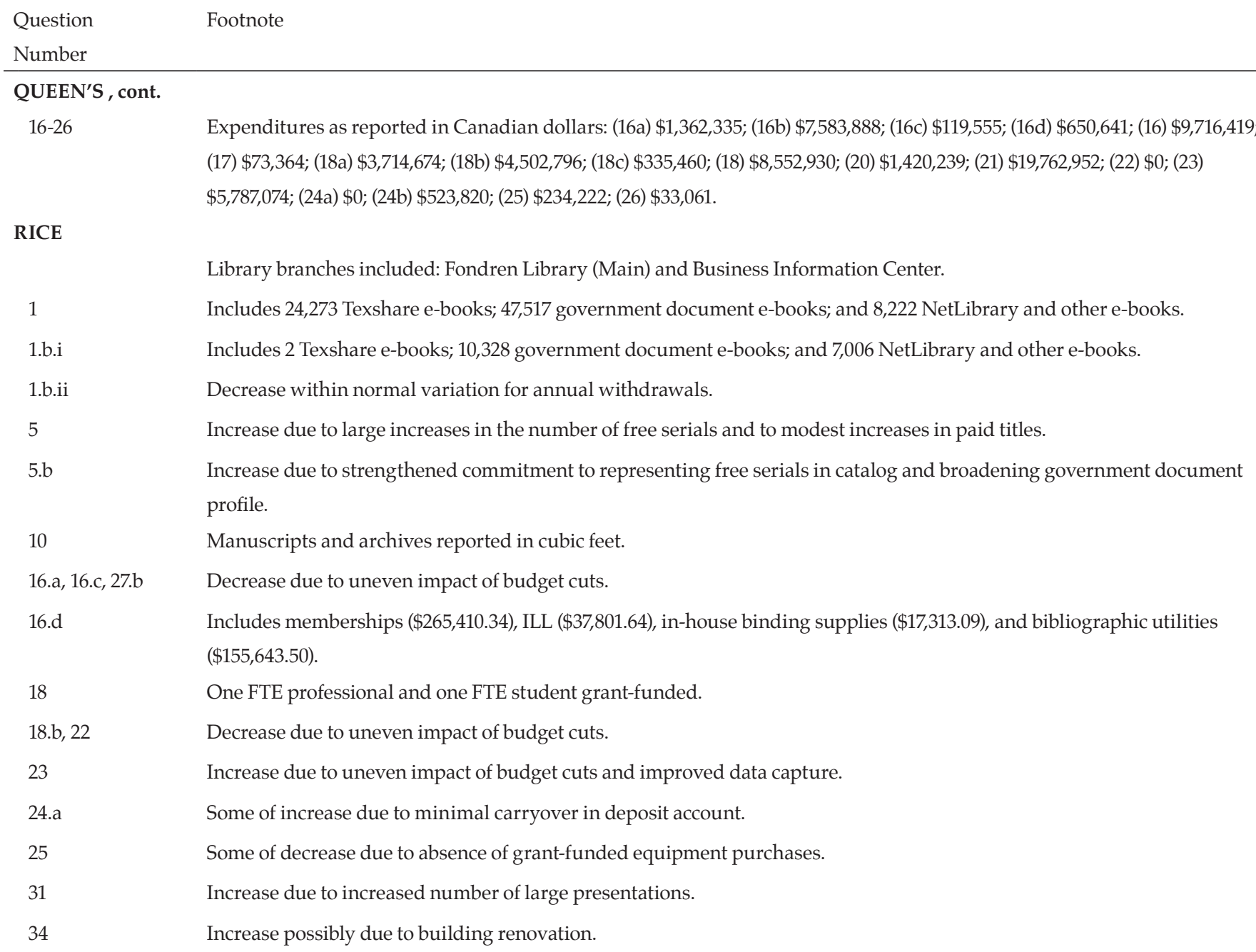

\section{ROCHESTER}

1.a

4

5.b.ii

6

18.a

20

27.a

28

30.a

31.a

$33-34,37-43$

Library branches included: Eastman School of Music Library.

Data from the Health Sciences Library are also included in the figures reported.

Volumes held June 30, 2008 revised to 3,715,385 which includes 3,446,277 volumes from the Main Library and 269,108 volumes from the Health Sciences Library. Figure reflects the addition of 14,144 e-books.

The basis of the count for 2,787 of the volumes is physical.

Includes 77 government documents.

Some are included under freely accessible.

Includes grant funding.

Does not include facilities. 2007-2008 figure included facilities.

Includes 6 grant positions.

Includes 4 from the Eastman School of Music Library.

120 based on sampling.

2,600 based on sampling.

Includes reserves. 
Question Footnote

Number

RUTGERS

1.a

Library branches included: Two Law Libraries, services to a non-Rutgers medical school, and campuses in Camden, Newark, and New Brunswick.

Volumes held June 30, 2008 revised to 4,529,916 which includes 3,839,518 volumes from the Main Library, 321,256 volumes from the Camden Law Library, and 369,142 volumes from the Newark Law Library.

1.b.ii

$11-14$

16.a

Performed inventory and weeding projects in several libraries resulting in an increase in numbers of volumes withdrawn.

Audiovisual Materials figures cannot be disaggregated. Total audiovisual is 154,034.

Increase due to increased expenditures for one-time costs and purchase of database back-files.

\section{SASKATCHEWAN}

All figures are as of 04/30/2009.

Library branches included: Education, Engineering, Murray (Main), Natural Sciences, and Veterinary Medicine Libraries.

Library branches NOT included: Theological College libraries on campus that are not part of the Library system.

Data from the Law and Health Sciences Library are also included in the figures reported.

1

1.

$16-26$

16.a-16.b

17

18.a

20

1.a

2

\section{SOUTHERN CALIFORNIA}

Library branches included: All libraries at the University of Southern California.

Data from the Law and Health Sciences Libraries are also included in the figures reported. Our approach to reporting has changed with our 2008-2009 submission. Consequently, we see several dramatic variances from 2007-2008's submission where our data gathering process changed. In addition, 2007-2008 data were incorrectly aggregated, so comparison with 2008-2009 results may not reflect actual variances.

5.b.i 2008-2009 figure decreases to zero for the Main Library because 2007-2008 reported figure did not match definition. Figure reflects the combined total consortial serials titles currently received but not purchased for the Law and Health Sciences Library.

2009-2009 increase results from an improved counting methodology. 
Question Footnote

Number

SOUTHERN CALIFORNIA, cont.

5.b.iv 2008-2009 figure decreased to zero because government document serials are now included in totals for "Current Serials" (Q5.b.ii) and change reflects more accurate methodology.

9

11

16.a

16.c

18.c

20

21

24.a

26

27.b

30

31

32

39

\section{SOUTHERN ILLINOIS}

1.a

1.b-1.b.i

3

$5 . b$

12

\section{SUNY-ALBANY}

1

3

17

26
Prior years' reported figures were incorrect. 2008-2009 figure is based on a Sirsi count of records.

2008-2009 increase results from an improved counting methodology.

Increase results from a one-time 17\% infusion to the monograph 2008-2009 budget for prepayment of approval plan. An increase in materials costs also contributes to the difference.

The increase results from a change in reporting methodology. 2007-2008 figures were not broken out so that the appropriate amount could be recorded on this line. Other Library Materials expenditures were included in Q20, Other Operating Expenses.

Change results from modification to student work force and student worker pay structure.

2007-2008 Other Operating Expenditures were overstated by $\$ 2,399,685$. See next footnote for additional explanation. The difference between $2007-2008$ and 2008-2009 should be a decrease of $\$ 3,234,321$ or $48.55 \%$ (\$6,661,770-\$3,427,449=\$3,234,321).

The difference in total library expenditures between 2007-2008 and 2008-2009 is \$2,810,268. 2007-2008 expenditures were overstated by $\$ 2,399,685$. Using 2008-2009 methodology, the total amount expensed in 2007-2008 was $\$ 27,526,214$. The Libraries received a budget increase between 2007-2008 and 2008-2009 allowing for a spending increase of 10.21\%. 2008-2009 increase is due, in part, to outsourcing cataloging to OCLC during the period.

Figure includes payments for ILL that were previously reported elsewhere.

To address potential financial issues spawned by global economic concerns, USC implemented a staff hiring freeze beginning November 2008, which remains in effect one year later.

For the first time, 2008-2009 figure includes a count of orientation sessions offered during the period in addition to other types of instruction sessions.

For the first time, 2008-2009 figure includes a count of participants in orientation sessions offered during the period in addition to participants who attended other types of instruction sessions.

Local IM counted for the first time in 2008-2009. All virtual reference increased this year, especially chat, which increased significantly.

2007-2008 figure included preclinical and clinical School of Medicine faculty. Per definition, they are excluded in 2008-2009.

Data from the Law and Medical Library are also included in the figures reported.

Volumes held June 30, 2008 revised to 3,134,360 which includes 2,747,367 volumes from the Main Library, 215,099 volumes from the Law Library, and 171,894 volumes from the Medical Library. Figure also includes 38,289 e-books from CREDO, Shaw-Shoemaker, and MyiLibrary.

Fewer bound volumes added to the collection due to cancellation of print serials. Also, in previous years, e-books were included in the volumes added, but this year the instructions said to add them to the previous year's total.

Decrease is explained by the fact that in 2007-2008, an e-book package was purchased that included over 4,500 volumes. Increase is due to our linkresolver vendor, Ex Libris, tracking more open access or freely available journals in their database. Decrease due to withdrawal of items.

Includes 77,390 e-books, of which 58,458 are U.S. government documents.

Includes 1,118 e-books.

Excludes theses and dissertations.

Includes bibliographic utility fees. 
Question Footnote

Number

SUNY-ALBANY, cont.

32

\section{SUNY-BUFFALO}

Data from the Law and Health Sciences Library are also included in the figures reported.

1.a

1.b

5

$16 . c$

16.d

22

\section{SUNY-STONY BROOK}

Library branches included: Math/Astronomy/Physics, Chemistry, Music, Marine and Atmospheric Sciences, Science and Technology, and Southampton.

Data from the Health Science Library are also included in the figures reported.

1.a

16

16.d

17

40

\section{SYRACUSE}

1.b.i

32

TEMPLE

1.a

1.b.i

1.b.ii

3

18.b

New method of counting, using READ software, results in lower counts. The READ Scale is a six-point scale used for recording qualitative statistics related to user queries. The READ Scale count is closer to a 1:1 ratio of patron to question transactions. The scale places an emphasis on the skills, knowledge, techniques, and tools used by the librarian during a specific reference transaction.

Volumes held June 30, 2008 revised to 3,681,102.

A large number of e-books were cataloged this year.

Variances from 2007-2008 figure due to better, more accurate counting methodology.

This is large due to some big one-time database purchases.

The amount usually reported here is no longer being provided.

This is large due to one-time purchases.

Volumes held June 30, 2008 revised to 2,265,566 which includes 1,991,940 volumes from the Main Library and 273,626 volumes from the Health Science Library.

Includes research and foundation funds and NYS Coordinated Collection Development Grant $(\$ 143,488)$.

Includes bibliographic utilities (\$281,900), ILL and document delivery $(\$ 31,561)$, freight $(\$ 17,440)$, off-site storage $(\$ 66,560)$, and memberships $(\$ 102,344)$.

Includes funds from NYS Preservation Grant $(\$ 8,876)$.

Figure is for all campuses.

Library branches included: Main and Law.

This figure now includes electronic books.

Reference statistics for 2007-2008 were under-reported. That year the figure was actually 49,082.

Library branches included: On main campus - Science, Engineering \& Architecture Library (SEAL), Charles L. Blockson Afro-American Collection; On other campuses: Ambler Library and Harrisburg Library.

Data from the Law Library and the Health Sciences Libraries are also included in the figures reported.

Volumes held June 30, 2008 revised to 3,700,645 which includes 3,111,135 volumes from the Main Library, 445,941 volumes from the Law Library, and 143,569 volumes from the Health Sciences Libraries. Revised from 2007-2008 figure to include the following records added: 59,249 e-book records added as part of one-time downloads during 2008-2009; plus 435 ,857 e-book records added between 2000-2001 and 2007-2008 but not reported earlier.

Includes 318 monographic e-books reported in Q3.

More weeding was done in 2008-2009 than in previous years.

Includes 318 monographic e-books per Q3 definition. Does not include 59,249 e-books added during 2008-2009 as one-time downloads. These 59,249 e-books are reported in Q1a.

Decrease in overall support staff expenditures from 2007-2008 figure is due to change in the tracking of Law Library support staff expenditures. 
Question Footnote

Number

TEMPLE, cont.

20

Decrease from 2007-2008 figure due to decline in renovation-related expenditures.

\section{TENNESSEE}

1.a

5

5.b.ii

16.d

18.c, 27.c

29

TEXAS

1

$1 . a$

16

$18 . c$

31

37

TEXAS A\&M

3

5.a-5.b

10

Library branches included: UT Libraries-Knoxville and UT Space Institute Library-Tullahoma.

Data from the Law Library and the Health Sciences Libraries are also included in the figures reported.

Volumes held June 30, 2008 revised to 3,278,642 which includes 2,715,382 volumes from the Main Library, 342,537 volumes from the Law Library, and 220,723 volumes from the Health Sciences Library. For the University Libraries, the volumes held as of June 30, 2008 was adjusted downward to remove internal processing records that did not accurately reflect end-user catalog items.

Before deduplication, figure for total Univ Libs/UTSI titles purchased was 37,151, which included 34,220 electronic titles and 2,931 print titles. Figure for total medical titles not purchased was 17,896.

Increase is due to University Libraries reporting this in 2008-2009 but not in 2007-2008.

Increase is due to the timing of payments to SOLINET.

Due to budget cuts, number of student assistants decreased from 40 to 28 at University Libraries.

The University Libraries are open 148 hours and the UT Space Institute Library is open 40 hours, but the UTSI hours occurred within the hours of operation for the University Libraries.

All figures are as of 08/31/2009.

Library branches included: University of Texas at Austin Libraries, the Briscoe Center for American History, the Tarlton Law Library, and the Humanities Research Center.

All figures are as of August 31, 2009 and include the University of Texas at Austin Libraries, the Briscoe Center for American History, the Tarlton Law Library, and the Humanities Research Center.

Volumes held June 30, 2008 revised to 9,760,046, which includes 8,969,440 volumes from the Main Library and 790,606 volumes from the Law Library. Figure adjusted to add e-books, including: EBL, MyiLibrary, eBrary, Gale Virtual Reference, ARTFL, CIAO, LION, Evans Early American Imprints, Making of Modern Law, Sabin Americana, Making of America, Oxford Reference Books online, Gerritsen, History eBook (now Humanities), OECD, World Bank eLibrary, Annual Reviews, Si Ku Quan Shu, Old English Corpus, Heritage Quest, Stat Reference, Thesaurus Linguae Graecae, and CHANT (ancient Chinese texts).

Includes total expenditures for library materials of $\$ 13,934,326$ by the University Libraries, $\$ 1,445,494$ by the Tarlton Law Library, and \$2,047,254 by the Humanities Research center.

Includes federal work-study funds.

Includes users of online tutorials.

Does not include EdD degrees.

All figures are as of 08/31/2009.

Library branches included: Sterling C. Evans Library, Library Annex, Cushing Memorial Library, Medical Sciences Library, West Campus Library, Policy Sciences \& Economics Library, Technical Reference Center, Jack K. Williams Library, and Texas A\&M University at Qatar Library.

Reflects purchase of 96,552 e-books.

Algorithm changed and is the primary reason for variances in 2008-2009 serials data.

Previous years' data were estimates. In 2008-2009, in addition to assessing and weeding the collection, it was physically measured. Both of these factors contributed to the variance from the 2007-2008 figure. 
Question Footnote

Number

TEXAS TECH

$1 . a$

\section{TORONTO}

1

TULANE

1.a

UTAH

1.a

1.b.ii

Library branches included: Library branches include: Harrington Library TTUHSC - Amarillo, Montes-Gallo Library of the Health Sciences TTUHSC-El Paso, Library of the Health Sciences TTUHSC-Odessa, Preston Smith HSC Library Lubbock, Architecture Library, Museum Library, Geosciences Library, Vietnam Archives Library, Southwest Collections/Special Collections Library.

Data from the Law Library and the Health Sciences Libraries are also included.

Volumes held revised to 2,625,804 which includes 2,104,341 volumes from the Main Library, 211,538 volumes from the Law Library, and 309,925 volumes from the Health Sciences Libraries. Increase from previous year due to correction of holdings at the Vietnam Library.

All figures are as of 04/30/2009.

Library branches included: The Main Libraries; Campus and Federated \& Affiliated Libraries: Architecture, Astronomy, Business, Chemistry, Criminology, Dentistry, Earth Sciences, Engineering \& Computer Sciences, Fine Art, Industrial Relations, Information Studies Forum, Innis College, Knox College, Law, Massey, Music, New College, Physics, Inst. of Policy Analysis, Regis College, Royal Ontario Museum, St. Augustine Seminary, St. Michael's College, Trinity College, University College, U of T Schools, Victoria College. Branch Campuses libraries include: the U of T Mississauga, and U of T Scarborough. Hospital Libraries include: Baycrest, Bloorview Kids Rehab, Mt. Sinai Hospital, St. Michael's Hospital, Toronto Rehab.

Includes monographic series and government documents, as well as 196,627 new e-books.

There is an increase in the number of electronic serials, but our title deduping procedures in 2007-2008 were not accurate. $\mathrm{U}$ of T Scarborough now open 24 hours on weekends.

Expenditures as reported in Canadian dollars: (16a) \$8,604,228; (16b) $\$ 12,603,285$; (16c) $\$ 5,988,061 ;$ (16d) $\$ 0$; $(16) \$ 27,195,574 ;(17)$ $\$ 360,254$; (18a) $\$ 16,412,874 ;$ (18b) \$16,253,345; (18c) \$4,685,293; (18) \$37,351,512; (20) \$5,801,026; (21) \$70,708,366; (22) \$1,435,220; (23) $\$ 11,918,185 ;(24 a) \$ 0 ;(24 b) \$ 0 ;(25) \$ 3,583,214 ;(26) \$ 145,240$.

2007-2008 figure was incorrectly reported. Should have been 312,933.

Library branches included: Turchin Business Library and Nadine Vorhoff Library at the Newcomb Women's Center.

Data from the Law and Medical Library are also included in the figures reported.

Volumes held June 30, 2008 revised to 3,891,843 which includes 3,417,665 volumes from the Main Library, 315,830 volumes from the Law Library, and 158,348 volumes from the Medical Library. Figure for the Main Library was revised to 3,417,665. Includes 601,476 e-books

Volumes added gross is an estimate. Excludes large one-time collection that was purchased to replenish volumes lost after hurricane Katrina.

Includes 601,476 e-books. The large number of monographic volumes purchased is related to huge purchases of e-books to replace a portion of the materials destroyed as a result of Hurricane Katrina in 2005. Over 400,000 volumes were withdrawn in 2005-2006, and delays in insurance settlements pushed off replacement until now.

Data from the Law and Health Sciences Library are also included in the figures reported.

Volumes held June 30, 2008 revised to 3,367,811, which includes 2,923,838 volumes from the Main Library, 244,730 volumes from the Law Library, and 199,243 volumes from the Health Sciences Library.

Increase due to the continual withdrawal of duplicate copies.

Serial title counts and expenditures reflect a shift from print to electronic.

Reflects increased purchase of electronic access to material. 


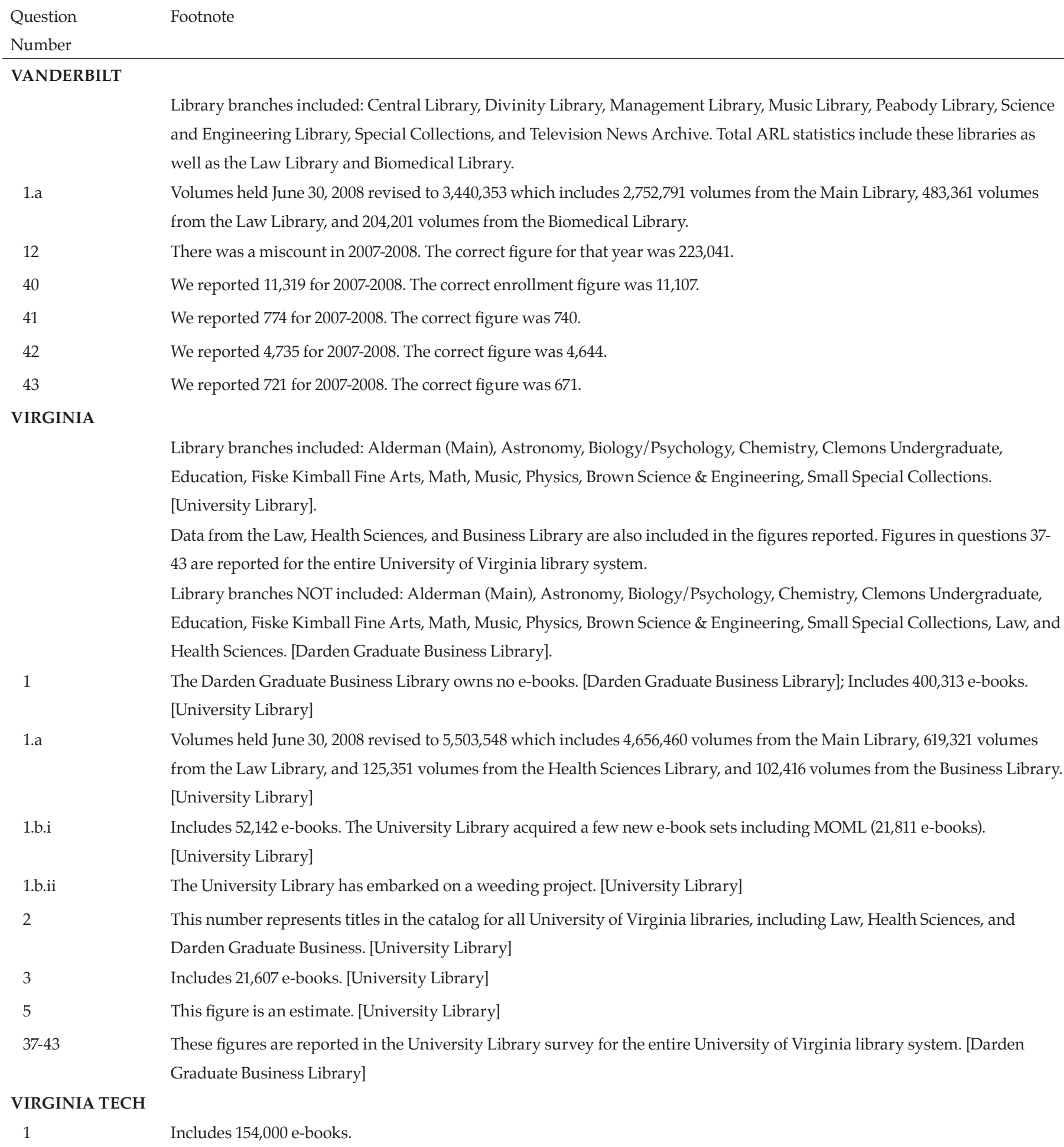

Library branches included: Central Library, Divinity Library, Management Library, Music Library, Peabody Library, Science and Engineering Library, Special Collections, and Television News Archive. Total ARL statistics include these libraries as well as the Law Library and Biomedical Library.

Volumes held June 30, 2008 revised to 3,440,353 which includes 2,752,791 volumes from the Main Library, 483,361 volumes from the Law Library, and 204,201 volumes from the Biomedical Library.

There was a miscount in 2007-2008. The correct figure for that year was 223,041.

We reported 11,319 for 2007-2008. The correct enrollment figure was 11,107.

We reported 774 for 2007-2008. The correct figure was 740 .

We reported 4,735 for 2007-2008. The correct figure was 4,644.

We reported 721 for 2007-2008. The correct figure was 671.

\section{VIRGINIA}

1

\section{VIRGINIA TECH}

Library branches included: Alderman (Main), Astronomy, Biology/Psychology, Chemistry, Clemons Undergraduate, Education, Fiske Kimball Fine Arts, Math, Music, Physics, Brown Science \& Engineering, Small Special Collections. [University Library].

Data from the Law, Health Sciences, and Business Library are also included in the figures reported. Figures in questions 3743 are reported for the entire University of Virginia library system.

Library branches NOT included: Alderman (Main), Astronomy, Biology/Psychology, Chemistry, Clemons Undergraduate, Education, Fiske Kimball Fine Arts, Math, Music, Physics, Brown Science \& Engineering, Small Special Collections, Law, and Health Sciences. [Darden Graduate Business Library].

The Darden Graduate Business Library owns no e-books. [Darden Graduate Business Library]; Includes 400,313 e-books. [University Library]

Volumes held June 30, 2008 revised to 5,503,548 which includes 4,656,460 volumes from the Main Library, 619,321 volumes from the Law Library, and 125,351 volumes from the Health Sciences Library, and 102,416 volumes from the Business Library. [University Library] Includes 52,142 e-books. The University Library acquired a few new e-book sets including MOML (21,811 e-books). [University Library] The University Library has embarked on a weeding project. [University Library]

This number represents titles in the catalog for all University of Virginia libraries, including Law, Health Sciences, and Darden Graduate Business. [University Library] Includes 21,607 e-books. [University Library] This figure is an estimate. [University Library] These figures are reported in the University Library survey for the entire University of Virginia library system. [Darden Graduate Business Library]

Includes 154,000 e-books.

\section{WASHINGTON}

Library branches included: Includes University of Washington (UW) Seattle (Main, Health Sciences, and Law), UW Bothell and UW Tacoma libraries. [Main Library]

Includes 404,725 e-books. [Main Library]

Includes 17,308 e-books added this year. [Main Library] 
WASHINGTON STATE

Library branches included: Washington State University (WSU) Vancouver, WSU Tri-Cities, WSU Energy Library, and WSU Riverpoint Campus Library.

1.a Volumes held June 30, 2008 revised to 2,342,754. Includes 3,184 volumes omitted from 2007-2008 count.

5 Since collection of the 2007-2008 statistics, many thousands of records for existing active titles have been added to the database from which this figure is gathered. The figure reported for 2007-2008 understates the true number of titles held.

\section{WASHINGTON U.-ST. LOUIS}

Library branches included: Art \& Architecture, Biology, Business, Chemistry, Earth Sciences, East Asian, Math, Music, Physics, and Social Work.

\section{WASHINGTON U.-ST. LOUIS, cont.}

Data from the Law and Health Science Library are also included in the figures reported.

1

1.a

20

39

\section{WATERLOO}

1.a

1.b.i

1.b.ii

3

5.b.ii

16-26

16.a

16.d

18

WAYNE STATE

10
Includes 358,667 e-books.

Volumes held June 30, 2008 revised to 4,224,887 which includes 3,455,152 volumes from the Main Library, 470,199 volumes from the Law Library, and 299,536 volumes from the Health Science Library.

Includes fringe benefits of $\$ 2,054,525$.

This figure reflects 907 faculty members at the Hilltop Campus and 954 at the Medical School (non-clinical). This figure in past survey years only reflected the undergraduate faculty.

All figures are as of 04/30/2009.

Library branches included: Includes the Musagetes Architecture Library, Optometry Learning Resource Centre, and the TriUniversity Storage Annex (University of Waterloo materials only).

Volumes held revised to 2,294,020. Adjusted to include one-time bulk-load of 7,605 e-book records.

Adjusted to include one-time bulk-load of 7,605 e-book records.

Increase due to a major weeding project initiated during the year.

Includes 19,664 e-books and 1,038 e-theses. The library acquired a large number of e-books in 2007-2008 and suspended the purchase of new monographs from Oct 2008 to April 2009. The combination of these two factors explains the decrease in monograph purchases.

Count extracted from SFX database.

Expenditures as reported in Canadian dollars: (16a) \$872,578; (16b) \$5,570,593; (16c) \$95,774; (16d) \$373,849; (16) \$6,912,794;

(17) $\$ 58,454$; (18a) $\$ 2,726,943 ;(18 b) \$ 3,761,353 ;$ (18c) $\$ 710,299 ;(18) \$ 7,198,595 ;(20) \$ 1,487,544 ;(21) \$ 15,657,387 ;(22) \$ 163,458 ;(23)$ $\$ 4,312,820$; (24a) \$15,536; (24b) \$0; (25) \$366,043; (26) \$65,253.

Some e-book expenditures included in other lines. The Library suspended the purchase of new monographs from Oct 2008 until April 2009.

Includes taxes, shipping, evaluations, ScholarsPortal and e-reserves.

Salary and personnel figures are current to April 30, 2009.

All figures are as of 09/30/2009.

Library branches included: The Reuther Archives of Labor and Urban Affairs, the Shiffman Medical Library, and the Neef Law Library. The Medical and Law library statistics cannot be disaggregated from the main statistics because the Medical and Law collections are an integral part of the Main Library.

This is an estimated measurement. In the past year, the manuscript and archives collection has been reevaluated. We now estimate that the size of the collection in 2007-2008 was 69,000 linear feet. 
Question Footnote

Number

WAYNE STATE, cont.

22

23

33

34

39

\section{WESTERN ONTARIO}

All figures are as of 04/30/2009.

Library branches included: Johnston Library, Education Library, John and Dotsa Bitove Family Law Library, Music Library, Allyn and Betty Taylor Library, and The D.B. Weldon Library.

$1 . b$

2

5

5.b.i

9

11

12

13

14

$16-26$

$22,24 . a, 25-26$

35

Very few one-time electronic resources were purchased during 2008-2009 due to a flat acquisition budget.

This drop in expenditures reflects a reduction of resource duplication achieved through canceling bundled title packages. Our initial circulation count for 2007-2008 was incorrect. The true count was 99,688. The original count contained ILL transactions.

Our total circulation count for 2007-2008 was incorrect. The true count was 153,016. The original count contained ILL transactions.

The count submitted in the 2007-2008 Survey did not exclude "faculty for preclinical and clinical medicine." This count excludes faculty in our School of Medicine.

Includes 503,898 e-books.

Includes 22,913 e-books.

Includes e-book titles.

Total includes 22,913 e-books from the following vendors: Adam Matthew Digital, Alexander Press, American Council of Learned Societies, American Geophysical Union, BMJ Publishing Group, Center for Research Libraries (CRL), Cognet, CRKN, EBSCO, Electronic Book Library (EBL), Factiva, Gale, Hein Online, Intersociety Council for Pathology Information Inc., JSTOR, Knowledge Ontario, McGraw Hill,Morgan \& Claypool, NetLibrary, Organisation for Economic Co-operation and Development (OECD), Ovid, Portland Press Ltd., RAND, Readex, Sage, Scholarsportal, Springer, Taylor \& Francis, Thomson Gale, United Nations Office of Legal Affairs, Treaty Section, United States Government Publications Office (USGPO), World Advertising Research Center, and World Bank.

Changes were made during the past year in coding of the order records to provide greater clarity for staff working in the data base. This resulted in changes to how the data can be retrieved and the numbers generated. Current numbers for serials counts in questions $5 \mathrm{a}$ and $5 \mathrm{~b}$ are believed to be more accurate than in previous years.

Consortial titles included in Q5.a.i, as the University directly funds the purchase through the consortium.

Two departmental/faculty libraries are currently transitioning to Western Libraries, and it is anticipated that this count may change in 2010.

With the current transitioning of a departmental map library on campus to Western Libraries, this count will change in future.

Count includes the London Free Press Negative Collection only.

Count includes Music Library only audio materials. Increased growth in audio and video supports newer programming by filling in gaps.

Count includes Music Library only film and video.

Expenditures as reported in Canadian dollars: (16a) \$2,259,357; (16b) \$9,623,600; (16c) \$0; (16d) \$0; (16) \$11,882,957; (17) \$130,254;

(18a) $\$ 4,505,189 ;$; (18b) \$4,140,056; (18c) \$331,943; (18) \$8,977,188; (20) \$748,464; (21) \$21,738,863; (22) \$48,361; (23) \$7,277,571; (24a) $\$ 223,743 ;(24 b) \$ 0 ;(25) \$ 653,111 ;(26) \$ 15,293$.

Centrally funded and administered at Western Libraries.

The number of requests decreased over the past year as a result of service enhancements to teaching hospitals, and consortial agreements among university libraries in Ontario providing electronic copies of materials to more scholars in the province.

The University is currently growing its graduate student enrollment. 
Library branches included: Main (GLS) (General Library System): Archives, Art, Astronomy, Biology, Business, Chemistry, College, Geography, Geology \& Geophysics, Math, Memorial, Music, Physics, Primate, School of Library \& Information Science (SLIS), Social Science, Social Work, Special Collections, Steenbock (Agriculture \& Life Sciences), and Wendt (English). Non-GLS Libraries: Health (Ebling), Law, Map, Education (Merit), Primate, and Wisconsin Historical Society (American history).

Library branches NOT included: American Indian Studies Program, Arboretum, Cooperative Children's Book Center, Center for Demography \& Ecology, Chicano \& Latin Studies, Journalism Reading Room, Max Kade German-American Institute, LGBT Campus Center, Limnology Reading Room, Learning Support Services, Morgridge Center Library, Plant Pathology Library, Space Library, Trout Lake Collection, University Communications Library, and the Wisconsin's Water Library.

Volumes held June 30, 2008 revised to 8,209,929 which includes 7,451,303 volumes from the Main Library, 441,394 volumes from the Law Library, and 317,232 volumes from the Health Science Library. The increase reflected is based upon an amendment for Merit Library (formerly CIMC education), the Wisconsin Historical Society, and a purchase by the Law Library. In 2007-2008 bound journals and separate juv. issues in the Merit Library were not counted as volumes previously due to misinterpretation of the definition of "volume" in the ARL instructions. In 2007-2008 there was a one-time increase reflected in the volumes held in the Wisconsin Historical Society based upon pre-1976 publications that were purchased through a MARCIVE GPO download. In 2008-2009 the Law Library purchased the Cengage collection, Making of Modern Law, representing 25,048 volumes. The total number of volumes withdrawn includes 18,304 reserve items. In 2007-2008 reserved items were not included in the total.

$5,668,964$ is the number of unique print titles listed in our OPAC, but there are additional titles in our card catalog that are not in the OPAC but were cataloged, classified and made ready for use.

In September 2008, Geology Library began a retrocon project which added 1,902 records for government documents. These records were mostly for state documents. The remaining documents will be part of a retrocon project in 2009-2010. Number of physical units may include accompanying material. The number of physical units may include accompanying material. Additionally, a Geology retrocon project added 2,697 bibliographic records for US Geological Survey maps transferred from the Map Library. Geology staff processed, barcoded, and added 1,688 maps for these records. As the remaining maps associated with the retrocon project are barcoded, they will be added to the map holdings. Furthermore, this number still excludes a significant number of transferred maps for which records have yet to be added. As records are added and maps processed, they will be included in the statistics. As it stands, the total count of Cartographic materials includes 7,172 items, 40 of which are new maps and 1,688 of which are maps from the retrocon/transfer project.

Increases are the result of conversions from paper or paper \& electronic to electronic only, and the splitting of paper and electronic expenditures. If a title was in paper and electronic formats, in many cases the cost was historically identified with paper. Also, there were some significant electronic backfile purchases in 2009.

Increased expenditures are due to continual increases in document delivery and inter-library loan requests. A notable change is the reporting source for campus document delivery and inter-library (ILL) loan expenditures. Prior ARL reports merely reflected OCLC costs which was not an accurate representation of all related services. Document delivery and ILL's comprehensive expenditures encompass OCLC costs billed through WiLS, article procurement, and relevant invoices for borrowing and lending. The change in data scope for these expenditures has resulted in a substantial increase reported for the fiscal year 2008-2009.

30-31 Reduction from 2007-2008 figure is based upon current review of campus reporting for small-group instruction and consultations. 
Question Footnote

Number

WISCONSIN, cont.

32

34

YALE

1.a

2

5.b.iii

6

9

21

34

39

YORK

1.a

2

14

$16-26$

29

33

\section{BOSTON PUBLIC LIBRARY}

4
There is no way to accurately report the campus-wide number of reference transactions. No sampling was done during the 2008-2009 academic year.

There was an error in the 2007-2008 report for total circulation transactions. The figure 896,335 was calculated in error, the correct total for circulation (initials and renewals, excluding reserves) is 804,182 for that period.

Library branches included: Arts Library, Bass Library, Beinecke Rare Book and Manuscript Library; Chemistry Library, Classics Luibrary, Divinity Library, Engineering and Applied Science Library, Forestry and Environmental Studies Library, Geology Library, Kline Science Library, Law Library, Lewis Walpole Library, Mathematics Library, Medical Library, Music Library, Social Science Library and Information Services.

Library branches NOT included: Preservation.

Volumes held June 30, 2008 revised to 12,283,594 which includes 10,590,443 volumes from the Main Library, 1,230,913 volumes from the Law Library, and 462,238 volumes from the Medical Library.

This figure represents the number of bibliographic records in the Library's online catalog.

Included in print count.

This is included in serials print counts.

Since 2002-2003, we note the number added only in the notes field. Count of computer files added in 2008-2009 is 3,161. We are not reporting a cumulative figure.

Increase may be due to purchases of large e-book, journal backfile and digitized newspaper purchases made in 2008-2009.

The methodology for extracting circulation data from LIS was more accurate this year than past, and figures reported include Law Library (different LIS).

Number includes both full-time ladder and non-ladder faculty at the University.

All figures are as of 04/30/2009.

Library branches included: Scott Library, Bronfman Business Library, Steacie Science \& Engineering Library, Frost Library (Glendon).

Data from the Law Library are also included in the figures reported.

Figure includes 2,272,355 volumes and 293,545 e-books.

Volumes were erroneously reported in 2007-2008 as 2,810,235. Volumes held revised to 2,833,749 which includes 2,491,798 volumes from the Main Library and 341,951 volumes from the Law Library.

We do not count sets of monographs separately. Therefore, our title count and volume count will be the same.

The 2007-2008 figure was too high.

Expenditures as reported in Canadian dollars: (16a) \$2,619,083; (16b) \$8,586,877; (16c) \$248,236; (16d) \$109,425; (16) \$11,563,621;

(17) $\$ 145,952 ;$; (18a) \$6,011,465; (18b) \$5,348,726; (18c) $\$ 1,094,863 ;(18) \$ 12,455,054 ;(20) \$ 3,396,000 ;(21) \$ 27,560,627 ;(22) \$ 774,992 ;$

(23) \$6,406,964; (24a) \$162,566; (24b) \$0; (25) \$399,676; (26) \$15,132.

Starting in February 2009, we instituted 24/5 hours in Scott Library.

We suspect that reserves were included with some of the circulation figures submitted to us in the past, and the initial loans were probably artificially inflated by reserves.

Volumes held June 30, 2008 revised to 7,631,990.

The basis of our volume count is both physical and bibliographic. 
Question Footnote

Number

LIBRARY \& ARCHIVES CANADA

All figures are as of 03/31/2009.

1,1.a-1.b Only one sector could provide this information.

4

Only one sector could provide this information. Also, count is in bibliographic (Resource center).

5.b, 5.b.iii

5.b.iv

This information is not available yet.

The tool used previous years to estimate the number of government documents is no longer available. A new tool is currently in development.

$16-26$

Expenditures as reported in Canadian dollars: (16a) \$552,247; (16b) \$626,015; (16c) \$132,725; (16d) \$206,157; (16) \$1,517,144; (17)

$\$ 0 ;$ (18a) \$60,948,363; (18b) \$26,893,118; (18c) \$752,872; (18) \$88,594,353; (20) \$0; (21) \$90,111,497; (22) \$0; (23) \$43,813; (24a) \$0; (24b)

$\$ 0 ;(25) \$ 9,891,990 ;(26) \$ 11,300$.

25

Fewer funds were available for hardware and software in 2008-2009.

28 We removed the Employee resource center.

33 We do not reserve documents or distinguish initial from renewals.

\section{LIBRARY OF CONGRESS}

All figures are as of 09/30/2009.

Library branches included: Library Services (national library functions), including 6 overseas offices, and Law Library of Congress.

Library branches NOT included: National Library Service for the Blind and Physically Handicapped.

1.a Volumes held revised to 21,218,408.

1.b.i Includes 313,182 monograph volumes and classified serial issues estimated to be the equivalent of 54,416 bound volumes of serials.

5.b.iii Includes serial titles received through copyright registration and/or deposit.

16.a LC receives most publications in U.S. through copyright deposit and therefore does not purchase them.

16.b Includes $\$ 1$ million for postage and handling and $\$ 100,000$ for replacement of damaged serial issues.

16.d Services of bibliographic service representatives in China, Greece, Mongolia, and West Africa.

18.a Includes salaries and wages for all employees in Library Services (national library functions) and Law Library of Congress.

23 Includes $\$ 23,764$ for OCLC FirstSearch access.

30 Includes Visitor Services Office and Library Services directorates for acquisitions/bibliographic access, collections, and preservation.

31 Decrease was expected because in 2008 LC initiated the self-guided Library of Congress Experience, eliminating need for some guided tour participation.

\section{NATIONAL AGRICULTURAL LIBRARY}

All figures are as of 09/30/2009.

Library branches included: DC Reference Center.

18-18.c Includes fringe benefits.

\section{NATIONAL LIBRARY OF MEDICINE}

All figures are as of 09/30/2009.

There are 27 service points at the National Library of Medicine (NLM) at the following NLM facilities: History of Medicine Desk, Circulation Desk, Reference Desk, Photocopy Service desk, Telephone service desk.

2

We are unable to easily compile this information. Will need to develop queries to report next year.

18-18.c

Includes fringe benefits. 


\title{
NATIONAL LIBRARY OF MEDICINE, cont.
}

Includes: Main RR Ref desk, History of Medicine RR ref desk, Circulation Desk, Photocopy service desk, and Customer service telephone desk.

\section{NEW YORK PUBLIC LIBRARY}

1.b

5

16,20

18

23

25

\section{NEW YORK STATE LIBRARY}

17

25

32

35

\section{SMITHSONIAN}

Decrease due to ordering fewer materials.

Decrease due to reduction in serials purchased.

Decrease due to budget reduction.

Contractual obligations affected the 2008-2009 figure.

A policy change affected the 2008-2009 figure.

Figure includes cost of new ILS system.

Expenditures reduced due to delays and budget and contract process.

Budgetary controls prohibited purchase of equipment.

Decrease due to the exclusion of directional questions, which were included in the 2007-2008 count.

Joined LAND service and uploaded on-line ILL request form.

\begin{abstract}
All figures are as of 09/30/2009.
Library branches included: Smithsonian Institution (SI) Libraries has a total of 20 libraries located throughout the museums and research institutes of the Smithsonian Institution. Included are: National Air and Space Museum Library, Washington, DC; National Museum of American History, Washington, DC; National Museum of Natural History Library, Washington, DC; National Postal Museum Library, Washington, DC; National Zoological Park Library, Washington, DC; Smithsonian American Art Museum, National Portrait Gallery Library, Washington, DC; Smithsonian Environmental Research Center Library, Edgewater, Maryland; Anacostia Museum and Center for African American History and Culture Library, Washington, DC; Botany and Horticulture Library, Washington, DC; Cooper-Hewitt National Design Museum Library, New York, New York; Dibner Library of the History of Science and Technology, Washington, DC; Earl S. Tupper Library Smithsonian Tropical Research Institute, Republic of Panama; Freer Gallery of Art and Arthur M. Sackler Gallery Library, Washington, DC; Hirshhorn Museum and Sculpture Garden Library, Washington, DC; John Wesley Powell Library of Anthropology, Washington, DC; Joseph F. Cullman, 3rd Library of Natural History, Washington, DC; Museum Studies and Reference Library, Washington, DC; Museum Support Center Library, Suitland, Maryland; Vine Deloria Jr. Library, National Museum of the American Indian, Suitland, Maryland; Warren M. Robbins Library, National Museum of African Art, Washington DC. In 2007-2008, SI Libraries' senior management approved changing the method of counting number of physical volumes held to include all items that have barcodes and item records - not title-level. This includes all serials (bound \& unbound) that have a barcode attached, and anything else that we barcode. We consider this a physical count as barcodes have been physically applied to each volume. We have not reported a number for this question in past years. This year, our data-crunching librarian discovered that previously the program was counting only government SERIAL documents (a total of 287 documents, reported in Q5b. iv for 2008-2009). She updated the program to include government MONOGRAPHIC documents and found quite a few (52,752 - mostly received through our gift and exchange program). Note that these are numbers for US-ONLY government documents. Includes costs for OCLC bibliographic utilities only.
\end{abstract}

4

8 
Question Footnote

Number

SMITHSONIAN, cont.

17

20
No new money approved for binding contract from Federal or Trust allocations. This small amount came from a gift fund for the Anthropology Library.

Includes staff travel, training, cataloging, digitizing, collection care supplies and services, general, preservation and automation supplies, equipment purchases, memberships, equipment maintenance, and miscellaneous contracts. It does NOT include health and transit benefits, building maintenance, public programs, exhibitions and development expenditures. 



\section{ARL Member Libraries as of January 1, 2010}

The Association of Research Libraries (ARL) represents the interests of 124 libraries that serve major North American research institutions. The ARL Statistics and Measurement program is organized around identifying, collecting, analyzing, and distributing quantifiable information describing the characteristics of research libraries.

\begin{tabular}{|c|c|c|}
\hline Institution & Category & Full Name of Institution \\
\hline Alabama & $S$ & University of Alabama \\
\hline Alberta & $\mathrm{C}$ & University of Alberta \\
\hline Arizona & S & University of Arizona \\
\hline Arizona State & $S$ & Arizona State University \\
\hline Auburn & $S$ & Auburn University \\
\hline Boston & $\mathrm{P}$ & Boston University \\
\hline Boston College & $\mathrm{P}$ & Boston College \\
\hline Brigham Young & $\mathrm{P}$ & Brigham Young University \\
\hline British Columbia & $\mathrm{C}$ & University of British Columbia \\
\hline Brown & $\mathrm{P}$ & Brown University \\
\hline Berkeley, California & $S$ & University of California, Berkeley \\
\hline Calgary & $\mathrm{C}$ & University of Calgary \\
\hline California, Davis & S & University of California, Davis \\
\hline California, Irvine & $S$ & University of California, Irvine \\
\hline California, Los Angeles & S & University of California, Los Angeles \\
\hline California, Riverside & S & University of California, Riverside \\
\hline California, San Diego & $S$ & University of California, San Diego \\
\hline California, Santa Barbara & S & University of California, Santa Barbara \\
\hline Case Western Reserve & $\mathrm{P}$ & Case Western Reserve University \\
\hline Chicago & $\mathrm{P}$ & University of Chicago \\
\hline Cincinnati & $S$ & University of Cincinnati \\
\hline Colorado & $\mathrm{S}$ & University of Colorado \\
\hline Colorado State & S & Colorado State University \\
\hline Columbia & $\mathrm{P}$ & Columbia University \\
\hline Connecticut & S & University of Connecticut \\
\hline Cornell & $\mathrm{P}$ & Cornell University \\
\hline Dartmouth & $\mathrm{P}$ & Dartmouth College \\
\hline Delaware & S & University of Delaware \\
\hline Duke & $\mathrm{P}$ & Duke University \\
\hline Emory & $\mathrm{P}$ & Emory University \\
\hline Florida & $S$ & University of Florida \\
\hline Florida State & S & Florida State University \\
\hline George Washington & $\mathrm{P}$ & George Washington University \\
\hline Georgetown & $\mathrm{P}$ & Georgetown University \\
\hline Georgia & $\mathrm{S}$ & University of Georgia \\
\hline Georgia Tech & S & Georgia Institute of Technology \\
\hline Guelph & $\mathrm{C}$ & University of Guelph \\
\hline Harvard & $\mathrm{P}$ & Harvard University \\
\hline Hawaii & $S$ & University of Hawaii \\
\hline
\end{tabular}

\section{Location}

Tuscaloosa, Alabama

Edmonton, Alberta

Tucson, Arizona

Tempe, Arizona

Auburn, Alabama

Boston, Massachusetts

Boston, Massachusetts

Provo, Utah

Vancouver, British Columbia

Providence, Rhode Island

California, Berkeley

Calgary, Alberta

Davis, California

Irvine, California

Los Angeles, California

Riverside, California

La Jolla, California

Santa Barbara, California

Cleveland, Ohio

Chicago, Illinois

Cincinnati, Ohio

Boulder, Colorado

Fort Collins, Colorado

New York, New York

Storrs, Connecticut

Ithaca, New York

Hanover, New Hampshire

Newark, Delaware

Durham, North Carolina

Atlanta, Georgia

Gainesville, Florida

Tallahassee, Florida

Washington, DC

Washington, DC

Athens, Georgia

Atlanta, Georgia

Guelph, Ontario

Cambridge, Massachusetts

Honolulu, Hawaii 


\begin{tabular}{|c|c|c|}
\hline Institution & Category & Full Name of Institution \\
\hline Houston & S & University of Houston \\
\hline Howard & $\mathrm{P}$ & Howard University \\
\hline Illinois, Chicago & $S$ & University of Illinois at Chicago \\
\hline Illinois, Urbana & S & University of Illinois at Urbana \\
\hline Indiana & $S$ & Indiana University \\
\hline Iowa & $\mathrm{S}$ & University of Iowa \\
\hline Iowa State & S & Iowa State University \\
\hline Johns Hopkins & $\mathrm{P}$ & Johns Hopkins University \\
\hline Kansas & S & University of Kansas \\
\hline Kent State & $S$ & Kent State University \\
\hline Kentucky & $S$ & University of Kentucky \\
\hline Laval & $\mathrm{C}$ & Laval University \\
\hline Louisiana State & $S$ & Louisiana State University \\
\hline Louisville & $S$ & University of Louisville \\
\hline McGill & $\mathrm{C}$ & McGill University \\
\hline McMaster & $\mathrm{C}$ & McMaster University \\
\hline Manitoba & $\mathrm{C}$ & University of Manitoba \\
\hline Maryland & $S$ & University of Maryland \\
\hline Massachusetts & S & University of Massachusetts \\
\hline MIT & $\mathrm{P}$ & Massachusetts Institute of Technology \\
\hline Miami & $\mathrm{P}$ & University of Miami \\
\hline Michigan & S & University of Michigan \\
\hline Michigan State & S & Michigan State University \\
\hline Minnesota & $S$ & University of Minnesota \\
\hline Missouri & S & University of Missouri \\
\hline Montreal & $\mathrm{C}$ & University of Montreal \\
\hline Nebraska & S & University of Nebraska-Lincoln \\
\hline New Mexico & S & University of New Mexico \\
\hline New York & $\mathrm{P}$ & New York University \\
\hline North Carolina & $S$ & University of North Carolina \\
\hline North Carolina State & $S$ & North Carolina State University \\
\hline Northwestern & $\mathrm{P}$ & Northwestern University \\
\hline Notre Dame & $\mathrm{P}$ & University of Notre Dame \\
\hline Ohio & $S$ & Ohio University \\
\hline Ohio State & S & Ohio State University \\
\hline Oklahoma & S & University of Oklahoma \\
\hline Oklahoma State & $S$ & Oklahoma State University \\
\hline Oregon & $S$ & University of Oregon \\
\hline Pennsylvania & $\mathrm{P}$ & University of Pennsylvania \\
\hline Pennsylvania State & $S$ & Pennsylvania State University \\
\hline Pittsburgh & $S$ & University of Pittsburgh \\
\hline Princeton & $\mathrm{P}$ & Princeton University \\
\hline Purdue & S & Purdue University \\
\hline Queen's & $\mathrm{C}$ & Queen's University \\
\hline
\end{tabular}

\section{Location}

Houston, Texas

Washington, DC

Chicago, Illinois

Urbana, Illinois

Bloomington, Indiana

Iowa City, Iowa

Ames, Iowa

Baltimore, Maryland

Lawrence, Kansas

Kent, Ohio

Lexington, Kentucky

Quebec, Quebec

Baton Rouge, Louisiana

Louisville, Kentucky

Montreal, Quebec

Hamilton, Ontario

Winnipeg, Manitoba

College Park, Maryland

Amherst, Massachusetts

Cambridge, Massachusetts

Coral Gables, Florida

Ann Arbor, Michigan

East Lansing, Michigan

Minneapolis, Minnesota

Columbia, Missouri

Montreal, Quebec

Lincoln, Nebraska

Albuquerque, New Mexico

New York, New York

Chapel Hill, North Carolina

Raleigh, North Carolina

Evanston, Illinois

Notre Dame, Indiana

Athens, Ohio

Columbus, Ohio

Norman, Oklahoma

Stillwater, Oklahoma

Eugene, Oregon

Philadelphia, Pennsylvania

University Park, Pennsylvania

Pittsburgh, Pennsylvania

Princeton, New Jersey

West Lafayette, Indiana

Kingston, Ontario 


\begin{tabular}{|c|c|c|c|}
\hline Institution & Category & Full Name of Institution & Location \\
\hline Rice & $\mathrm{P}$ & Rice University & Houston, Texas \\
\hline Rochester & $\mathrm{P}$ & University of Rochester & Rochester, New York \\
\hline Rutgers & S & Rutgers University & New Brunswick, New Jersey \\
\hline Saskatchewan & $\mathrm{C}$ & University of Saskatchewan & Saskatoon, Saskatchewan \\
\hline South Carolina & S & University of South Carolina & Columbia, South Carolina \\
\hline Southern California & $\mathrm{P}$ & University of Southern California & Los Angeles, California \\
\hline Southern Illinois & S & Southern Illinois University & Carbondale, Illinois \\
\hline SUNY-Albany & S & University at Albany, State University of New York & Albany, New York \\
\hline SUNY-Buffalo & S & University at Buffalo, State University of New York & Buffalo, New York \\
\hline SUNY-Stony Brook & S & State University of New York at Stony Brook & Stony Brook, New York \\
\hline Syracuse & $\mathrm{P}$ & Syracuse University & Syracuse, New York \\
\hline Temple & S & Temple University & Philadelphia, Pennsylvania \\
\hline Tennessee & S & University of Tennessee & Knoxville, Tennessee \\
\hline Texas & S & University of Texas & Austin, Texas \\
\hline Texas A\&M & S & Texas A\&M University & College Station, Texas \\
\hline Texas Tech & S & Texas Tech University & Lubbock, Texas \\
\hline Toronto & $\mathrm{C}$ & University of Toronto & Toronto, Ontario \\
\hline Tulane & $\mathrm{P}$ & Tulane University & New Orleans, Louisiana \\
\hline Utah & S & University of Utah & Salt Lake City, Utah \\
\hline Vanderbilt & $\mathrm{P}$ & Vanderbilt University & Nashville, Tennessee \\
\hline Virginia & $\mathrm{S}$ & University of Virginia & Charlottesville, Virginia \\
\hline Virginia Tech & S & Virginia Polytechnic Institute \& State University & Blacksburg, Virginia \\
\hline Washington & S & University of Washington & Seattle, Washington \\
\hline Washington State & S & Washington State University & Pullman, Washington \\
\hline Washington U.-St. Louis & $\mathrm{P}$ & Washington University & St. Louis, Missouri \\
\hline Waterloo & $\mathrm{C}$ & University of Waterloo & Waterloo, Ontario \\
\hline Wayne State & S & Wayne State University & Detroit, Michigan \\
\hline Western Ontario & $\mathrm{C}$ & University of Western Ontario & London, Ontario \\
\hline Wisconsin & $\mathrm{S}$ & University of Wisconsin & Madison, Wisconsin \\
\hline Yale & $\mathrm{P}$ & Yale University & New Haven, Connecticut \\
\hline York & $\mathrm{C}$ & York University & North York, Ontario \\
\hline Boston Public Library & $\mathrm{N}$ & Boston Public Library & Boston, Massachusetts \\
\hline Canada Inst. SciTech Info. & $X$ & Canada Inst. for Scientific \& Technical Information & Ottawa, Ontario \\
\hline Center for Research Libraries & $\mathrm{N}$ & Center for Research Libraries & Chicago, Illinois \\
\hline Library of Congress & $\mathrm{N}$ & Library of Congress & Washington, DC \\
\hline National Agricultural Library & $\mathrm{N}$ & National Agricultural Library & Beltsville, Maryland \\
\hline Library and Archives Canada & $\mathrm{X}$ & Library and Archives Canada & Ottawa, Ontario \\
\hline Naionat. Library of Medicine & $\mathrm{N}$ & National Library of Medicine & Bethesda, Maryland \\
\hline New York Public Library & $\mathrm{N}$ & New York Public Library & New York, New York \\
\hline New York State Library & $\mathrm{N}$ & New York State Library & Albany, New York \\
\hline Smithsonian Institution & $\mathrm{N}$ & Smithsonian Institution & Washington, DC \\
\hline $\begin{array}{l}\text { S: US public university } \\
\text { C: Canadian university }\end{array}$ & \multicolumn{2}{|c|}{$\begin{array}{l}\text { P: US private university } \\
\text { X: Canadian nonuniversity }\end{array}$} & \\
\hline
\end{tabular}





\section{BIBLIOGRAPHY}

\section{PART A: Selected Articles from the ARL Newsletter}

“The ARL Membership Criteria Index."ARL: A Bimonthly Newsletter of Research Library Issues and Actions 197 (April 1998$): 9$.

"Assessing ILL/DD Services: New Cost-Effective Alternatives." ARL: A Bimonthly Report on Research Library Issues and Actions from ARL, CNI, and SPARC 236 (October 2004): 9.

Askew Waller, Consuella, and Kaylyn Hipps. “Using LibQUAL+ and Developing a Culture of Assessment in Libraries.” ARL: A Bimonthly Report on Research Library Issues and Actions from ARL, CNI, and SPARC 221 (April 2002): 10-11.

Case, Mary M. "The Impact of Serial Costs on Library Collections." ARL: A Bimonthly Report on Research Library Issues and Actions from ARL, CNI, and SPARC 218 (October 2001): 9.

. "A Snapshot in Time: ARL Libraries and Electronic Journal Resources." ARL: A Bimonthly Report on Research Library Issues and Actions from ARL, CNI, and SPARC 235 (August 2004): 1-10.

Case, Mary M., and Judith Matz. "Framing the Issue: Open Access." ARL: A Bimonthly Report on Research Library Issues and Actions from ARL, CNI, and SPARC 226 (February 2003): 8-10.

Case, Mary M., and Prudence Adler. "Promoting Open Access." ARL: A Bimonthly Report on Research Library Issues and Actions from ARL, CNI, and SPARC 220 (February 2002): 1-5.

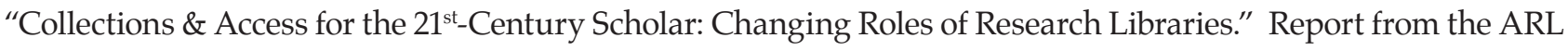
Collections \& Access Issues Task Force. ARL: A Bimonthly Report on Research Library Issues and Actions from ARL, CNI, and SPARC 225 (December 2002).

Cook, Colleen, Fred Heath, and Bruce Thompson. "A Brief LibQUAL+ Phase One Progress Report." ARL: A Bimonthly Report on Research Library Issues and Actions from ARL, CNI, and SPARC 219 (December 2001): 7.

Cook, Colleen, and Michael Maciel. “A Decade of Assessment at a Research-Extensive University Library Using LibQUAL+ ${ }^{\circledR}$." ARL: A Bimonthly Report on Research Library Issues and Actions from ARL, CNI, and SPARC 271 (August 2010): 4-12.

Crow, Raym. "The Case for Institutional Repositories: A SPARC Position Paper." ARL: A Bimonthly Report on Research Library Issues and Actions from ARL, CNI, and SPARC 223 (August 2002): 1-4.

Crowe, William. "The End of History? Reflections on a Decade." ARL: A Bimonthly Report on Research Library Issues and Actions from ARL, CNI, and SPARC 226 (February 2003): 12-13.

Davidson, Catherine, and Martha Kyrillidou. “The Value of Electronic Resources: Measuring the Impact of Networked Electronic services (MINES for Libraries ${ }^{\circledR}$ at the Ontario Council of University Libraries." ARL: A Bimonthly Report on Research Library Issues and Actions from ARL, CNI, and SPARC 271 (August 2010): 41-47.

Dowd, Jacqui. "LibQUAL $+{ }^{\circledR}$ and the "Library as Place" at the University of Glasgow." ARL: A Bimonthly Report on Research Library Issues and Actions from ARL, CNI, and SPARC 271 (August 2010): 13-20. 
"The Future of Human Resources in Canadian Libraries." ARL: A Bimonthly Report on Research Library Issues and Actions from ARL, CNI, and SPARC 240 (June 2005): 9.

Grosetta Nardini, Holly. "Building a Culture of Assessment." ARL: A Bimonthly Report on Research Library Issues and Actions from ARL, CNI, and SPARC 218 (October 2001): 11.

Groves, Richard. "Sharing Best Practices by Disseminating Assessment Results via the Web." ARL: A Bimonthly Report on Research Library Issues and Actions from ARL, CNI, and SPARC 236 (October 2004): 6.

Guedon, Jean-Claude. "Beyond Core Journals and Licenses: The Paths to Reform Scientific Publishing." ARL: A Bimonthly Report on Research Library Issues and Actions from ARL, CNI, and SPARC 218 (October 2001): 1-8.

Hahn, Karla. "The State of the Large Publisher Bundle: Findings from an ARL Member Survey." ARL: A Bimonthly Report on Research Library Issues and Actions from ARL, CNI, and SPARC 245 (April 2006).

Hipps, Kaylyn. “Diversity in the U.S. ARL Library Workforce." ARL: A Bimonthly Report on Research Library Issues and Actions from ARL, CNI, and SPARC 246 (June 2006): 1-2.

Johnson, Richard K. “Whither Competition?” ARL: A Bimonthly Report on Research Library Issues and Actions from ARL, CNI, and SPARC 217 (August 2001): 12-14.

Killick, Selena. "Service Quality Assessment with LibQUAL $+{ }^{\circledR}$ in Challenging Times: LibQUAL $+{ }^{\circledR}$ at Cranfield University." ARL: A Bimonthly Report on Research Library Issues and Actions from ARL, CNI, and SPARC 271 (August 2010): 21-24.

Kyrillidou, Martha. "The ARL Library Scorecard Pilot: Using the Balanced Scorecard in Research Libraries." ARL: A Bimonthly Report on Research Library Issues and Actions from ARL, CNI, and SPARC 271 (August 2010): 33-35.

. "ARL University Libraries' Spending Trends." ARL: A Bimonthly Report on Research Library Issues and Actions from ARL, CNI, and SPARC 242 (October 2005): 10.

. "The Future of Librarians in the U.S. Workforce." ARL: A Bimonthly Report on Research Library Issues and Actions from ARL, CNI, and SPARC 246 (June 2006): 5.

. "The Impact of Electronic Publishing on Tracking Research Library Investments in Serials." ARL: A Bimonthly Report on Research Library Issues and Actions from ARL, CNI, and SPARC 249 (December 2006): 6-7.

. "LibQUAL+TM in 2004." ARL: A Bimonthly Report on Research Library Issues and Actions from ARL, CNI, and SPARC 236 (October 2004): 6.

. “Library Value May Be Proven, If Not Self-Evident.” ARL: A Bimonthly Report on Research Library Issues and Actions from ARL, CNI, and SPARC 271 (August 2010): 1-3.

. "Reshaping ARL Statistics to Capture the New Environment." ARL: A Bimonthly Report on Research Library Issues and Actions from ARL, CNI, and SPARC 256 (February 2008): 9-11. 
. "Serials Trends Reflected in the ARL Statistics 2002-03." ARL: A Bimonthly Report on Research Library Issues and Actions from ARL, CNI, and SPARC 234 (June 2004): 14-15..

Kyrillidou, Martha, and William Crowe. "In Search of New Measures." ARL: A Bimonthly Newsletter of Research and Library Issues and Actions 197 (April 1998): 8-10.

Kyrillidou, Martha, and Kaylyn Hipps. "Symposium on Measuring Library Service Quality." ARL: A Bimonthly Report on Research Library Issues and Actions from ARL, CNI, and SPARC 215 (April 2001): 9-11.

Lippincott, Sarah, and Martha Kyrillidou. "How ARL University Communities Access Information: Highlights From LibQUAL+" ${ }^{\mathrm{TM}}$." ARL: A Bimonthly Report on Research Library Issues and Actions from ARL, CNI, and SPARC 236 (October 2004): 7-8.

Lynch, Clifford. "Institutional Repositories: Essential Infrastructure for Scholarship in the Digital Age." ARL: A Bimonthly Report on Research Library Issues and Actions from ARL, CNI, and SPARC 226 (February 2003): 1-7.

"Making Library Assessment Work." ARL: A Bimonthly Report on Research Library Issues and Actions from ARL, CNI, and SPARC 240 (June 2005): 9.

Mays, Regina, Carol Tenopir, and Paula Kaufman. "Lib-Value: Measuring Value and Return on Investment of Academic Libraries." ARL: A Bimonthly Report on Research Library Issues and Actions from ARL, CNI, and SPARC 271 (August 2010): 36-40.

Potter, William Gray, Colleen Cook, and Martha Kyrillidou. "ARL Profiles: Qualitative Descriptions of Research Libraries in the Early 21st Century." ARL: A Bimonthly Report on Research Library Issues and Actions from ARL, CNI, and SPARC 271 (August 2010): 25-32.

Shim, Wonsik "Jeff", Charles McClure, and John Carlo Bertot. "Measures and Statistics for Research Library Networked Services: ARL E-Metrics Phase II Report." ARL: A Bimonthly Report on Research Library Issues and Actions from ARL, CNI, and SPARC 219 (December 2001): 8-9.

Stuber, Peter. “Where does the Free Online Scholarship Movement Stand Today?” ARL: A Bimonthly Report on Research Library Issues and Actions from ARL, CNI, and SPARC 220 (February 2002): 9-15.

Unsworth, John M. "The Crisis in Scholarly Publishing in the Humanities." ARL: A Bimonthly Report on Research Library Issues and Actions from ARL, CNI, and SPARC 228 (June 2003): 1-4.

Wetzel, Karen, and Mary Jackson. "Portal Functionality Provided by ARL Libraries: Results of an ARL Survey." ARL: A Bimonthly Report on Research Library Issues and Actions from ARL, CNI, and SPARC 222 (June 2002): 7-9.

Wilder, Stanley. "New Hires in Research Libraries: Demographic Trends and Hiring Priorities." ARL: A Bimonthly Report on Research Library Issues and Actions from ARL, CNI, and SPARC 221 (April 2002): 5-8.

Young, Mark. "ARL Salary Survey Highlights." ARL: A Bimonthly Report on Research Library Issues and Actions from ARL, CNI, and SPARC 246 (June 2006): 4. 


\section{PART B: Related Books and Articles Published in Other Sources}

Arms, William. "Quality Control in Scholarly Publishing on the Web." The Journal of Electronic Publishing 8, no. 1 (August 2002).

Band, Jonathan. “The Three P's: A Tribute to Duane Webster." portal: Libraries and the Academy 9, no. 3 (2009): 367-74.

Baumol, W. J., and M. Marcus. Economics of Academic Libraries. Washington, DC: American Council of Education, 1973.

Bertot, John Carlo, Charles McClure, and Joe Ryan. Statistics and Performance Measures for Public Library Networked Services. Chicago: American Library Association, 2001.

Blixrud, Julia. "The Association of Research Libraries Statistics and Measurement Program: From Descriptive Data to Performance Measures." Proceedings from the $4^{\text {th }}$ Northumbria International Conference on Performance Measurement in Libraries and Information Services, edited by Joan Stein, Martha Kyrillidou, and Denise Davis. Washington, DC: Association of Research Libraries, 2002.

Branin, Joseph J. “Duane Webster's Contributions to Leadership Development in Research Libraries, 1970-2008." portal: Libraries and the Academy 9, no. 3 (2009): 349-54.

Carlson, Scott. "The Deserted Library: As Students Work Online, Reading Rooms Empty Out - Leading Some Campuses to Add Starbucks." The Chronicle of Higher Education, November 16, 2001.

Case, Mary M. “Scholarly Communication: ARL as a Catalyst for Change." portal: Libraries and the Academy 9, no. 3 (2009): 381-96.

Clapp, V. W. The Future of the Research Library. Urbana: University of Illinois Press, 1964.

Coffman, Steve. “Building Earth's Largest Library: Driving into the Future.” Searcher 7, no. 3 (March 1999).

Competition Commission of the United Kingdom. Reed Elsevier, PLC, and Harcourt General, Inc.: A Report on the Proposed Merger, presented to Parliament by the Secretary of State and Trade and Industry by Command of Her Majesty, July 2001.

Cook, Colleen, Fred Heath, Martha Kyrillidou, and Duane Webster. "The Forging of Consensus: A Methodological Approach to Service Quality Assessment in Research Libraries - the LibQUAL+ Experience." Proceedings from the $4^{\text {th }}$ Northumbria International Conference on Performance Measurement in Libraries and Information Services, edited by Joan Stein, Martha Kyrillidou, and Denise Davis. Washington, DC: Association of Research Libraries, 2002.

Cook, Colleen, Fred Heath, and Bruce Thompson. "Score Norms for Improving Library Service Quality: A LibQUAL+ study." portal: Libraries and the Academy 2 (2002): 13-26.

Cook, Colleen, Fred Heath, Bruce Thompson, and Russell Thompson. "LibQUAL+: Service Quality Assessment in Research Libraries." IFLA Journal 27 (2001): 264-68.

. The Search for New Measures: The ARL LibQUAL+Project-A Preliminary Report. Baltimore: Johns Hopkins University Press, 2001. Accessed at http://muse.jhu.edu/demo/pla/1.1cook.html on August 22, 2001. 
Cook, Colleen, Fred Heath, Bruce Thompson, and Duane Webster. “LibQUAL+TM Preliminary Results from 2002." Performance Measurement and Metrics 4, no. 1 (2003): 38-47.

Council on Library and Information Resources. A Different Approach to the Evaluation of Research Libraries. Research Brief 6. Washington, DC: The Council, 1998.

Cronenwett, Philip N., Kevin Osborn, Samuel A. Streit, eds. Celebrating Research: Rare and Special Collections from the Membership of the Association of Research Libraries. Washington, DC: Association of Research of Libraries, 2007.

Crow, Raym. “The Case for Institutional Repositories: A SPARC Position Paper.” Washington, DC: Scholarly Publishing \& Academic Resources Coalition, 2002.

Cummings, Anthony M., et al. University Libraries and Scholarly Communication: A Study Prepared for The Andrew W. Mellon Foundation. Washington, DC: Association of Research Libraries, 1992.

Cummings, M. M. The Economics of Research Libraries. Washington, DC: Council on Library Resources, 1986.

Dewey, Barbara I. “The Imperative for Diversity: ARL's Progress and Role." portal: Libraries and the Academy 9, no. 3 (2009): 355-62.

Downs, Robert B. “The Growth of Research Collections.” Library Trends: American Library History: 1876-1976 25 (July 1976): $55-80$. “Uniform Statistics for Library Holdings.” Library Quarterly 16 (January 1946): 63-69.

Drake, Miriam A. “Forecasting Academic Library Growth.” College and Research Libraries 37 (January 1976): 53-59.

Franklin, Brinley. “Duane Webster, Assessment Pioneer.” portal: Libraries and the Academy 9, no. 3 (2009): 339-48.

Franklin, Brinley, Colleen Cook, Martha Kyrillidou, and Bruce Thompson. "Library Investment Index-Why Is It Important?" Proceedings of the 2008 Library Assessment Conference: Building Effective, Sustainable, and Practical Assessment, August 4-7, 2008, Seattle, Washington. (Washington, DC: Association of Research Libraries, 2009): $147-54$.

Frazier, Kenneth. "The Librarians' Dilemma: Contemplating the Costs of the 'Big Deal,"' D-Lib Magazine 7, no. 3 (March 2001), viewed online at http://www.dlib.org/dlib/march01/frazier/03frazier.html.

Funk, Cara J. "ARL and Association 3.0: Ten Management Challenges." portal: Libraries and the Academy 9, no. 3 (2009): 405-10.

Gooden, Paul, Matthew Owen, and Sarah Simon. Scientific Publishing: Knowledge is Power. New York: Morgan Stanley, 2002.

Guthrie, Kevin M. “It Didn't Have to Be This Way: Entrepreneurship at ARL During Duane Webster's Tenure.” portal: Libraries and the Academy 9, no. 3 (2009): 411-18.

Haslett, K. Mark. "Canada, Copyright, and the Common Good." portal: Libraries and the Academy 9, no. 3 (2009): 375-80. 
Hayes, Robert M. Strategic Management for Academic Libraries: A Handbook. Westport, Conn.: Greenwood Press, 1993.

Heath, Fred. "A Salute to a Leader: ARL's Assessment Protocol Initiatives." portal: Libraries and the Academy 9, no. 3 (2009): 333-38.

Heath, Fred, Colleen Cook, Martha Kyrillidou, and Bruce Thompson. "ARL Index and Other Validity Correlates of LibQUAL+ Scores." portal: Libraries and the Academy 2 (2002): 27-42.

Hernon, Peter, and Ellen Altman. Assessing Service Quality. Chicago: American Library Association, 1998.

Hernon, Peter, and Robert E. Dugan. Action Plan for Outcomes Assessment in Your Library. Chicago: American Library Association, 2002.

Hernon, Peter, and John R. Whitman. Delivering Satisfaction and Service Quality. Chicago: American Library Association, 2001.

Hightower, Christy, Julie Sih, and Adam Tilgham. "Recommendations for Benchmarking Web Site Usage Among Academic Libraries." College and Research Libraries 59 (January 1998): 61-79.

Hiller, Steve, Martha Kyrillidou, and Jim Self, "Assessment in North American Research Libraries: a Preliminary Report Card." Performance Measurement and Metrics 7, no. 2 (2006): 100-6.

Jackson, Mary E. Measuring the Performance of Interlibrary Loan Operations in North American Research E College Libraries. Washington, DC: Association of Research of Libraries, 1998.

Jakubs, Deborah. “The Global Resources Program.” portal: Libraries and the Academy 9, no. 3 (2009): 327-32.

Johnson, Richard K. "A Question of Access: SPARC, BioOne, and Society-Driven Electronic Publishing." D-Lib Magazine (May 2000), accessed at http://www.dlib.org/dlib/may00/johnson/05johnson.html on August 21, 2001.

Johnson, Richard K, and Judy Luther. “The E-Only Tipping Point for Journals." Washington, DC: Association of Research of Libraries, 2007.

Kling, Rob, Lisa Spector, and Geoff McKim. “The Guild Model.” The Journal of Electronic Publishing 8, no. 1 (August 2002).

Kuhlman, A. F. “Two ARL Approaches to Counting Holdings of Research Libraries.” College and Research Libraries 21 (May 1960): 207-11.

Kyrillidou, Martha. “To Describe and Measure the Performance of North American Research Libraries." IFLA Journal 27 (2001, No. 4): 257-63.

. "From Input and Output Measures to Quality and Outcome Measures, or, from the User in the Life of the Library to the Library in the Life of the User." The Journal of Academic Librarianship 28, no. 1 (January-March 2002): $42-46$.

."Library Assessment as a Collaborative Enterprise." Resource Sharing and Information Networks 1/2 (Special 200506 issue on the theme "Creative Collaborations: Libraries Within Their Institutions and Beyond"): 73-87.

$172 \cdot$ ARL Statistics 2008-2009 
. "Research Library Trends: ARL Statistics." Washington, DC: Association of Research Libraries, 2001. Accessed at http://www.arl.org/stats/arlstat/jal99.html on August 22, 2001.

Kyrillidou, Martha, and Colleen Cook. "The Evolution of Measurement and Evaluation of Libraries: A Perspective from the Association of Research Libraries." Library Trends 56, no. 4 (Spring 2008): 888-909.

Kyrillidou, Martha, and Fred Heath, eds. “Measuring Service Quality.” Library Trends 49 (Spring 2001).

Lakos, Amos. "Culture of Assessment as a Catalyst for Organizational Culture Change in Libraries." Proceedings from the $4^{\text {th }}$ Northumbria International Conference on Performance Measurement in Libraries and Information Services, edited by Joan Stein, Martha Kyrillidou, and Denise Davis. Washington, DC: Association of Research Libraries, 2002.

Lavoie, Brian, Lynn Silipigni Connaway, and Lorcan Demsey. "Anatomy of aggregate collections: the example of Google Print for libraries" D-Lib Magazine 11, no. 9 (September 2005): http://www.dlib.org/dlib/september05/ lavoie/09lavoie.html.

Lynch, Clifford A., and Joan K. Lippincott. “Institutional Repository Deployment in the United States as of Early 2005.” D-Lib Magazine 11, no. 9 (September 2005): http://www.dlib.org/dlib/september05/lynch/09lynch.html .

Mekkawi, Mod. “The ARL Library Index as a Decision-Making Tool.” College and Research Libraries 43 (September 1982): 396-401.

Miller, Rush, and Sherrie Schmidt. "E-Metrics: Measures for Electronic Resources." Proceedings from the $4^{\text {th }}$ Northumbria International Conference on Performance Measurement in Libraries and Information Services, edited by Joan Stein, Martha Kyrillidou, and Denise Davis. Washington, DC: Association of Research Libraries, 2002.

Miller, Rush, Sherrie Schmidt, and Martha Kyrillidou. “New Initiatives in Performance Measures." Global Issues in $21^{\text {st }}$ Century Research Librarianship, edited by Sigrun Klara Hannesdottir. Helsinki: NORDINFO, 2002.

Molyneux, Robert E. The Gerould Statistics 1907/08 - 1961/62. Washington, DC: Association of Research Libraries, 1986.

. "Patterns, Processes of Growth, and the Projection of Library Size: A Critical Review of the Literature on Academic Library Growth." Library and Information Science Research 8 (January-March 1986): 5-28.

. "What Did Rider Do? An Inquiry into the Methodology of Fremont Rider's The Scholar and the Future of the Research Library." Libraries and Culture 29 (Summer 1994): 297-325.

Molyneux, Robert E., and Robert V. Williams. "Measuring the Internet." Annual Review of Information Science and Technology 34 (1999-2000): 287-339.

Morris, Dilys E., Collin B. Hobert, Lori Osmus, and Gregory Wool. “Cataloging Staff Costs Revisited." LRTS 44 (2000): 70-83.

Nitecki, Danuta, and Toni Olshen. “Developing Service Quality Measurement Skills Through an Online Course for Librarians." Proceedings from the $4^{\text {th }}$ Northumbria International Conference on Performance Measurement in Libraries and Information Services, edited by Joan Stein, Martha Kyrillidou, and Denise Davis. Washington, DC: Association of Research Libraries, 2002. 
O'Connor, Lisa, Carolyn Radcliff, and Julie Gedeon. “Applying Systems Design and Item Response Theory to the Problem of Measuring Information Literacy Skills." College and Research Libraries 63, no. 6 (2002): 528-43.

Piternick, George. "ARL Statistics—Handle With Care." College and Research Libraries 38 (September 1977): 419-23.

."Library Growth and Academic Quality." College and Research Libraries 24 (May 1963): 223-29.

Rider, Fremont. The Scholar and the Future of the Research Library. New York: Hadham Press, 1944.

Roche, Marilyn M. ARL/RLG Interlibrary Loan Cost Study. Washington, DC: Association of Research Libraries, 1993.

Rosenblatt, Susan. "Developing Performance Measures for Library Collections and Services." Chap. 18 in The Mirage of Continuity: Reconfiguring Academic Information Resources for the 21st Century, edited by Brian L. Hawkins and Patricia Battin. Washington, DC: Council on Library and Information Resources, 1998.

Shim, Wonsik, Charles McClure, and John Carlo Bertot. "Preliminary Statistics and Measures for ARL Libraries to Describe Electronic Resources and Services." Proceedings from the $4^{\text {th }}$ Northumbria International Conference on Performance Measurement in Libraries and Information Services, edited by Joan Stein, Martha Kyrillidou, and Denise Davis. Washington, DC: Association of Research Libraries, 2002.

Shulenburger, David. “The Political Climate for Higher Education During Duane Webster's ARL Tenure.” portal: Libraries and the Academy 9, no. 3 (2009): 363-66.

Stubbs, Kendon. "Access and ARL Membership Criteria." Proceedings of the $125^{\text {th }}$ Meeting of the Association of Research Libraries (1993): 117-22.

“Apples and Oranges and ARL Statistics." Journal of Academic Librarianship 14 (September 1988): 231-35.

. “Lies, Damned Lies, ... and ARL Statistics?" Paper presented at the $108^{\text {th }}$ meeting of Research Libraries: Measurement, Management, Marketing (May 1986): Minneapolis, MN.

. “On the ARL Library Index.” Paper presented at the $108^{\text {th }}$ meeting of Research Libraries: Measurement, Management, Marketing (May 1986): Minneapolis, MN.

. “University Libraries: Standards and Statistics." College and Research Libraries 42 (November 1981): 527-38.

Sullivan, Maureen. “Duane Webster's Contribution to Organization Development in Academic and Research Libraries." portal: Libraries and the Academy 9, no. 3 (2009): 313-16.

Thompson, Bruce. "Some Alternative Quantitative Library Activity Descriptions/Statistics that Supplement the ARL Logarithmic Index." Washington, DC: Association of Research Libraries, 2006.

Thompson, Bruce, Colleen Cook, and Russell Thompson. "Reliability and Structure of LibQUAL+ Scores." portal: Libraries and the Academy 2 (2002): 3-12.

van Westrienen, Gerard, and Clifford A. Lynch. "Academic Institutional Repositories: dEployment Status in 13 Nations as of Mid 2005" D-Lib Magazine 11, no. 9 (September 2005): http://www.dlib.org/dlib/september05/ westrienen/09westrienen.html. 
Vaughn, John C. "AAU and ARL: The Role of Partnerships and Collective Advocacy in Policy Development." portal: Libraries and the Academy 9, no. 3 (2009): 397-404.

Weiner, Sharon. “Library Quality and Impact: Is There a Relationship Between New Measures and Traditional Measures?" Journal of Academic Librarianship 31 (2005): 432-37.

West, Richard P. “The Coalition for Networked Information and the Rewards of Risk Taking." portal: Libraries and the Academy 9, no. 3 (2009): 317-25.

Wilder, Stanley J. The Age Demographics of Academic Librarians: A Profession Apart. A Report Based on Data from the ARL Annual Salary Survey. Washington, DC: Association of Research Libraries, 1995.

. Demographic Change in Academic Librarianship. Washington, D.C.: Association of Research Libraries, 2003.

Williams, Robert V. "The Making of Statistics of National Scope on American Libraries, 1836-1986: Purposes, Problems, and Issues." Libraries and Culture 26 (Spring 1991): 464-85.

Wysocki, Bernard. “Scholarly Journals' Premiere Status is Diluted by Web." Wall Street Journal, May 23, 2005; Page A1. 
